\title{
The diagnostic potential of 18F-Fluoride PET/CT in lumbar spinal fusion patients
}

\author{
Citation for published version (APA):
}

Peters, M. J. M. (2019). The diagnostic potential of 18F-Fluoride PET/CT in lumbar spinal fusion patients: early detection of pseudarthrosis and identification of pain generators after surgery. [Doctoral Thesis, Maastricht University]. GVO drukkers \& vormgevers B.V. https://doi.org/10.26481/dis.20191025mp

\section{Document status and date:}

Published: 01/01/2019

DOI:

10.26481/dis.20191025mp

Document Version:

Publisher's PDF, also known as Version of record

\section{Please check the document version of this publication:}

- A submitted manuscript is the version of the article upon submission and before peer-review. There can be important differences between the submitted version and the official published version of record.

People interested in the research are advised to contact the author for the final version of the publication, or visit the DOI to the publisher's website.

- The final author version and the galley proof are versions of the publication after peer review.

- The final published version features the final layout of the paper including the volume, issue and page numbers.

Link to publication

\footnotetext{
General rights rights.

- You may freely distribute the URL identifying the publication in the public portal. please follow below link for the End User Agreement:

www.umlib.nl/taverne-license

Take down policy

If you believe that this document breaches copyright please contact us at:

repository@maastrichtuniversity.nl

providing details and we will investigate your claim.
}

Copyright and moral rights for the publications made accessible in the public portal are retained by the authors and/or other copyright owners and it is a condition of accessing publications that users recognise and abide by the legal requirements associated with these

- Users may download and print one copy of any publication from the public portal for the purpose of private study or research.

- You may not further distribute the material or use it for any profit-making activity or commercial gain

If the publication is distributed under the terms of Article $25 \mathrm{fa}$ of the Dutch Copyright Act, indicated by the "Taverne" license above, 
The diagnostic potential of ${ }^{18} \mathrm{~F}$-fluoride PET/CT in lumbar spinal fusion patients

\author{
Early detection of pseudarthrosis and \\ identification of pain generators after surgery
}

Marloes Peters 


\section{Copyright}

(C) Marloes Peters, 2019. All rights reserved

\section{ISBN}

978-94-6332-561-5

Financial Support for the publication of this thesis was provided by:

Maastricht University

Stichting Kliniek en Wetenschap Orthopedie

\section{Cover Design}

Marloes Peters \& Amber van Amsterdam

\section{Layout}

Marloes Peters

\section{Printing}

GVO drukkers \& vormgevers B.V. 


\title{
The diagnostic potential of ${ }^{18} \mathrm{~F}$-fluoride PET/CT in lumbar spinal fusion patients
}

\author{
Early detection of pseudarthrosis and \\ identification of pain generators after surgery
}

ter verkrijging van de graad van doctor

aan de Universiteit Maastricht,

op gezag van de Rector Magnificus, prof. dr. Rianne M. Letschert

volgens het besluit van het College van Decanen,

in het openbaar te verdedigen

op vrijdag 25 oktober 2019 om 14.00 uur

door

Marloes Johanna Maria Peters

Geboren op 25 mei 1988 te Arnhem 


\section{Promotor}

Prof. dr. L.W. van Rhijn

\section{Copromotores}

Dr. P.C. Willems

Dr. B.T. Brans

\section{Beoordelingscommissie}

Prof. dr. M.W. de Haan (voorzitter)

Prof. dr. P. Habibovic

Prof. dr. B.J. van Royen (Amsterdam UMC)

Dr. ir. B. van Rietbergen (TU Eindhoven / UM)

Dr. A.W.J.M. Glaudemans (UMC Groningen) 


\section{Chapter 1}

General Introduction

\section{Chapter 2}

The diagnostic accuracy of imaging modalities to detect pseudarthrosis after spinal fusion - a systematic review and meta-analysis of the literature

\section{Chapter 3}

Pseudarthrosis after lumbar spinal fusion: the role of ${ }^{18} \mathrm{~F}$-fluoride PET/CT

\section{Chapter 4}

${ }^{18}$ F-fluoride PET/CT to identify pain generators after lumbar spinal fusion a retrospective analysis of symptomatic and asymptomatic patients

\section{Chapter 5}

Evaluation of a short dynamic ${ }^{18} \mathrm{~F}$-fluoride PET/CT scanning method to assess bone metabolic activity in spinal orthopedics

\section{Chapter 6}

${ }^{18} \mathrm{~F}$-fluoride PET/CT as an early predictor of bony fusion after posterior lumbar interbody fusion - a prospective study

\section{Chapter 7}

General Discussion

\section{Appendix}

Valorization

Summary

Nederlandse Samenvatting

List of Publications

Curriculum Vitae

Dankwoord 



\section{CHAPTER 1}

General Introduction 
Low back pain is a major problem globally. It is the leading cause of activity limitation and work absence [1, 2], as well as a huge economic burden. Costs associated with low back pain include direct costs for health-care and indirect costs because of work absenteeism [3]. The problem of low back pain is expected to continue to grow as a result of the aging and increasing population worldwide [1]. Low back pain is general nomenclature for several pathological conditions which distort normal spinal anatomy.

\section{Spinal anatomy}

The spine is an interconnected complex of bones, nerves, muscles, tendons and ligaments. Any of which can get damaged and cause pain, neurological symptoms or loss of mobility. The vertebrae are the building blocks of the spine. From the head downwards, the human vertebral column consists of seven cervical vertebrae, twelve thoracic vertebrae, five lumbar vertebrae and five sacral vertebrae (Figure 1A). Two vertebral bodies are referred to as a spinal segment, named for its upper and lower vertebra, for example L4 - L5 (fourth and fifth lumbar vertebrae). Although the morphology differs between spinal regions, each segment consists of the same structural elements (Figure 1B, 1C, 1D).

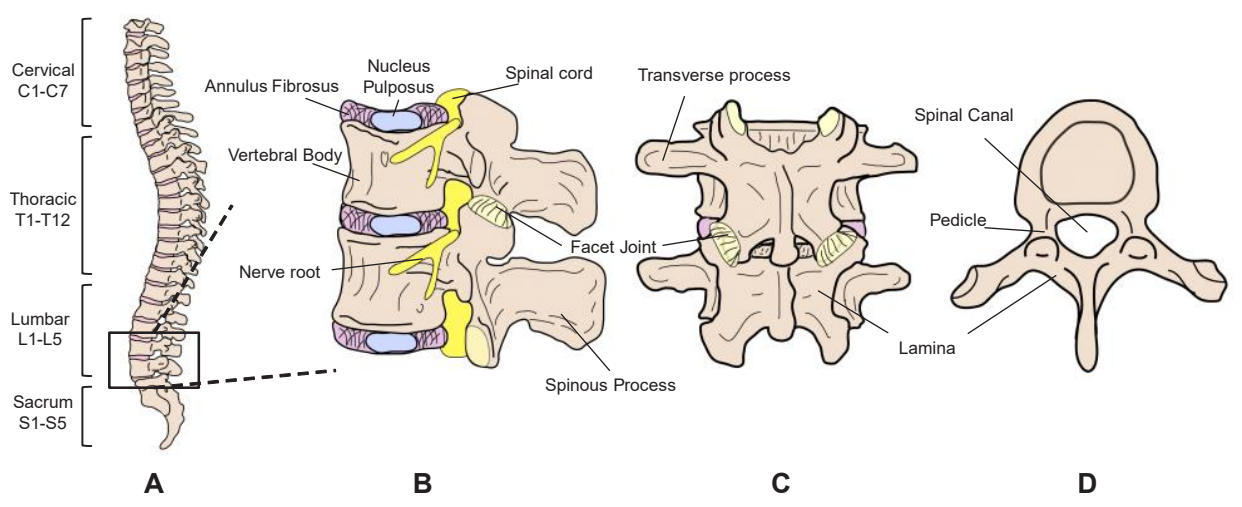

Figure 1. Schematic drawings of the human vertebral column. The spinal column consists of seven cervical vertebrae named C1 through C7; twelve thoracic vertebrae named T1 through T12; five lumbar vertebrae named L1 through L5; and five sacral vertebrae named S1 through S5 (A). A sagittal (B), coronal (C) and axial (D) view of a lumbar spinal segment including vertebral bodies, intervertebral discs composed of the nucleus pulposus and annulus fibrosus, facet joints, transverse and spinous processes, lamina, pedicles, spinal cord with branching nerve roots and the spinal canal.

Each spinal segment is comprised of two vertebral bodies, an intervertebral disc and paired facet joints. Intervertebral discs are soft cartilage-like discs between vertebrae that serve as shock absorbers and help facilitate movements of the spine. The discs are composed of an inner nucleus pulposus and an outer annulus fibrosus. Vertebral endplates form the boundaries between vertebral bodies and the intervertebral discs. The semi-permeable endplates allow for nutrient transport to the intervertebral discs. The 
facet joints allow for forward and backward extension as well as for twisting movements. The vertebral bodies and the vertebral arch, consisting of the lamina and pedicles, surround and protect the spinal cord, which runs behind the vertebral bodies within a canal from the neck down to the top of the lumbar spine. At each lumbar segment, nerve roots branch out from the spinal canal and exit the spine through gaps at the back of the vertebrae, the neuroforamina. Compression of the nerve roots in the foraminal opening may cause pain that can radiate along the path of the nerve to the lower extremities, which is known as radiculopathy. The lower the vertebra is located in the spinal column, the higher the load that is deposited on the vertebra and therefore the larger the vertebra is. The two lowest spinal segments in the lumbar spine: L4 - L5 and L5 - S1, carry the highest loads whilst allowing for the largest movements, making the area susceptible for injury and/or early degeneration.

Several alterations in the spinal anatomy can lead to low back pain with or without radiating leg pain:

- $\quad$ Degenerative disc disease:

Intervertebral discs are subject to degenerative changes over time. Degenerative discs may cause pain and/or neurological problems by inflammation, ingrowing sensory nerve endings and/or nerve root compression $[4,5]$.

- Disc herniation:

The nucleus pulposus of a disc can extrude outside the disc space and cause inflammation of nearby nerve roots, which can result in neurological problems.

- Facet joint degeneration:

Degeneration of the cartilage in between the bony parts of the facet joints can lead to bone-on-bone contact. As a result, the joint loses mobility and the surrounding tissue becomes inflamed which can cause pain. Bone spurs may form on the surface of the facet joints in response to the alterations, causing pain and instability. Bone spurs that compress nearby nerves, can cause neurological problems.

- Spondylolisthesis:

A patient suffers from spondylolisthesis when the upper vertebra of a segment partially slips over the lower vertebra. As a result, the spinal column loses stability and nerve roots can become compressed. Spondylolisthesis can be a consequence of degeneration of the intervertebral disc or facet joints, in which case the condition is referred to as degenerative spondylolisthesis. Spondylolisthesis can also occur as a result of fracture of the pars interarticularis which is the structure connecting the facet joints of two adjacent vertebrae, in which case the condition is referred to as lytic spondylolisthesis. 
It is important to understand the underlying cause of symptoms in patients with low back pain, in order to effectively treat the patient. Several imaging techniques have been developed to visualize the interior of the human body. Imaging can be used to confirm or rule out conditions that can cause pain. Common imaging modalities used in patients with low back pain include plain radiography, computed tomography (CT) and magnetic resonance imaging (MRI) (Figure 2).

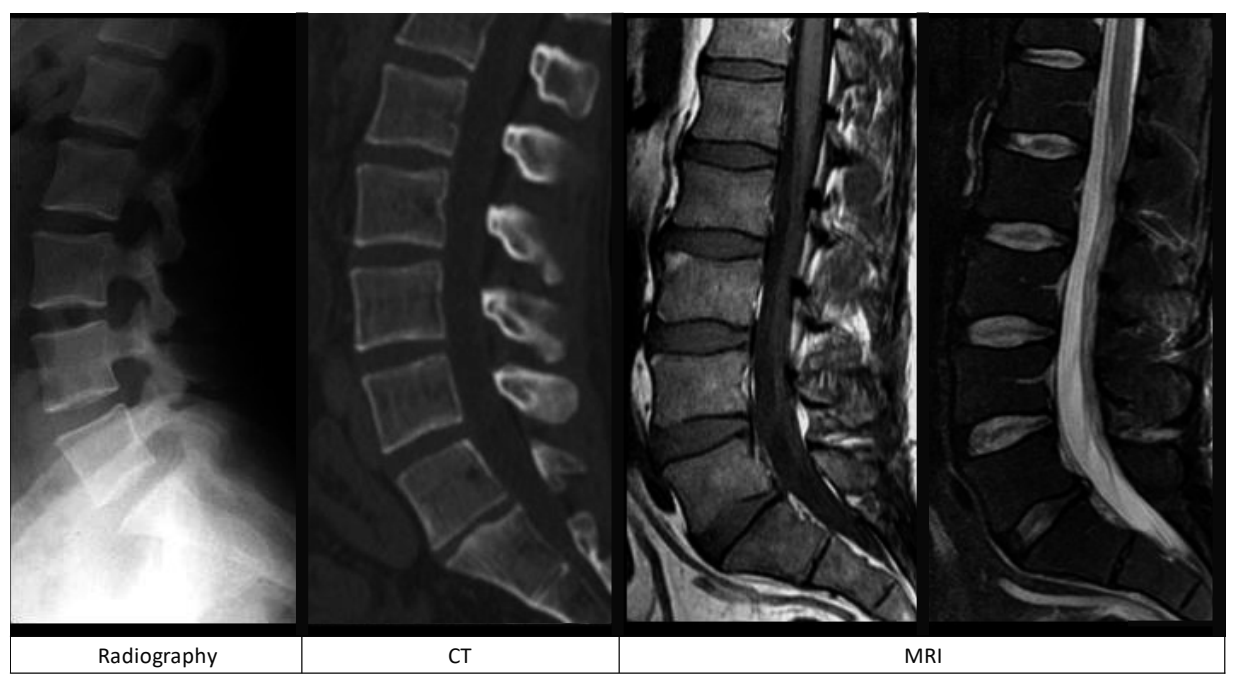

Figure 2 (adapted from [6]). From left to right: a human lumbar vertebral column imaged in sagittal orientation by means of radiography, CT and two types of imaging protocols on MRI. Radiography and CT visualize the bony structures while MRI is also able to image soft tissues, depending on the type of pulse sequence used.

In radiography, electromagnetic radiation (X-rays) is projected onto the patient from one side. The density and structural composition of the patient determine the amount of $X$ rays that pass through and fall onto a detector on the other side of the patient. Radiography provides a two-dimensional representation of the bony structures of the human spine and can be useful to detect misalignment of the spine, fractures, tumors and spondylolisthesis. CT is based on the same principle as radiography. However, instead of a single two-dimensional projection, $\mathrm{X}$-ray generators and detectors circulate around the patient to obtain a three-dimensional representation of the bony structures. This enables detection of conditions that in principle can also be visualized by radiography, but with higher accuracy. MRI uses magnetic fields with pulse sequences that influence specific types of tissue. MRI can produce high resolution three-dimensional images of bony structures as well as soft tissues such as muscles, ligaments, tendons and blood vessels, dependent on the pulse sequence that is used for imaging. MRI can be used in low back pain patients to detect disc degeneration, disc herniation, nerve root compression, infection, inflammation and tumors. 
Besides a condition that is visible on anatomical imaging modalities, psychological, social and genetic components can contribute to perceived pain also [3], which complicates the diagnosis of the patient. Setting a diagnosis in a patient with low back pain includes review of medical history, psychosocial circumstances, clinical examination and imaging. The medical history of the patient is reviewed to obtain information about the onset, site and severity of the pain as well as the duration of symptoms and comorbidities. Clinical examination is performed to assess any limitations in movement and neurological functions. Patient reported outcome measures (PROMs) are validated questionnaires, which can be used for the follow-up of the clinical status of patients. PROMs can quantify the burden of symptoms of a patient in terms of pain, loss of function and quality of life. Questionnaires are routinely used to supplement radiological findings and impact clinical decision making. PROMs commonly used to quantify the symptoms of low back pain patients are:

1. The Visual Analogue Scale (VAS) reliably quantifies back and leg pain [7]. Patients are asked to express the amount of pain in their back, right leg and left leg separately on a scale of 0 to 100 .

2. The Oswestry Disability Index (ODI) is the most commonly used outcome measure for low back pain and disability [8]. It assesses ten general daily tasks based on back related function [9].

3. The EuroQol (EQ)-5D is the most commonly used instrument to measure healthrelated quality of life in the Netherlands [10]. The questionnaire consists of five questions, each representing a domain (i.e. mobility, self-care, usual activities, pain/discomfort and anxiety/depression). The EQ-5D index score can be calculated based on a Dutch value set, representative of the Dutch population with regards to age and gender [11].

\section{Treatment of patients with low back pain}

Treatment of patients diagnosed with low back pain starts conservatively with physical therapy, pharmacological therapy (pain medication), and/or bracing. When these conservative measures do not have the desired effect, spinal surgery may be considered to stabilize the spine and/or to decompress nerve roots. Different types of spinal surgery can be performed dependent on the condition. The focus of the present thesis is spinal fusion. Spinal fusion, also referred to as spondylodesis, is a surgical procedure in which two or more vertebrae are fused by bony union to form a rigid segment. The first spinal fusion procedures were performed in 1911 in the United States by Hibbs and Albee to treat scoliosis and tuberculosis $[12,13]$. In the past decades, the indications for spinal fusion have evolved and currently include scoliosis, kyphosis, vertebral fractures, tumors and degenerative conditions such as disc degeneration and spondylolisthesis [14]. The 
rates of lumbar spinal fusion procedures in the United States have increased tremendously by $170.9 \%$ from 1998 to 2008 [15].

\section{Surgical technique: posterior lumbar interbody fusion}

Spinal fusion uses bone graft material to achieve definite bony fusion between vertebrae. The bone graft material can be inserted between the transverse processes of the vertebrae i.e. posterolateral fusion (Figure 3A) or between the vertebral bodies, i.e. interbody fusion. Lumbar interbody fusion was the focus of this thesis.

Different surgical approaches to reach the intervertebral disc space for interbody fusion are routinely employed. The most commonly employed approaches are the posterior, anterior, transforaminal, lateral and oblique approach, for which the lumbar interbody fusion procedure is subsequently named: PLIF - posterior lumbar interbody fusion, ALIF - anterior lumbar interbody fusion, TLIF - transforaminal lumbar interbody fusion, LLIF lateral lumbar interbody fusion, OLIF - oblique lumbar interbody fusion. PLIF is most commonly employed at our institution, and therefore, PLIF was the procedure subject to investigation in this thesis. PLIF starts with a posterior median incision, after which the spinous process and lamina of the affected spinal segment are exposed. The nerve roots are decompressed by removing bone or extruded disc tissue and the intervertebral disc space is excised. After thorough abrasion of the endplates, one or two cages can be inserted into the disc space. The cages are filled with graft material, for example autologous bone pieces from the removed lamina or artificial bone graft. Graft material is placed in the disc space surrounding the cages also. Screws are inserted through the pedicles of the vertebrae and connected to rods to provide primary stability to the segment. The instrumentation can share loads with the vertebrae and the cages including bone graft material to allow for bone healing and remodeling. The bony bridges that form subsequently can take over the load at a later stage. Figure 3B shows a schematic image of the spinal column after PLIF. The intervertebral cages contain tantalum markers that are radiopaque and therefore allow radiographic visualization during follow-up. Figure 4 shows radiographs of a human vertebral column before and after PLIF. 

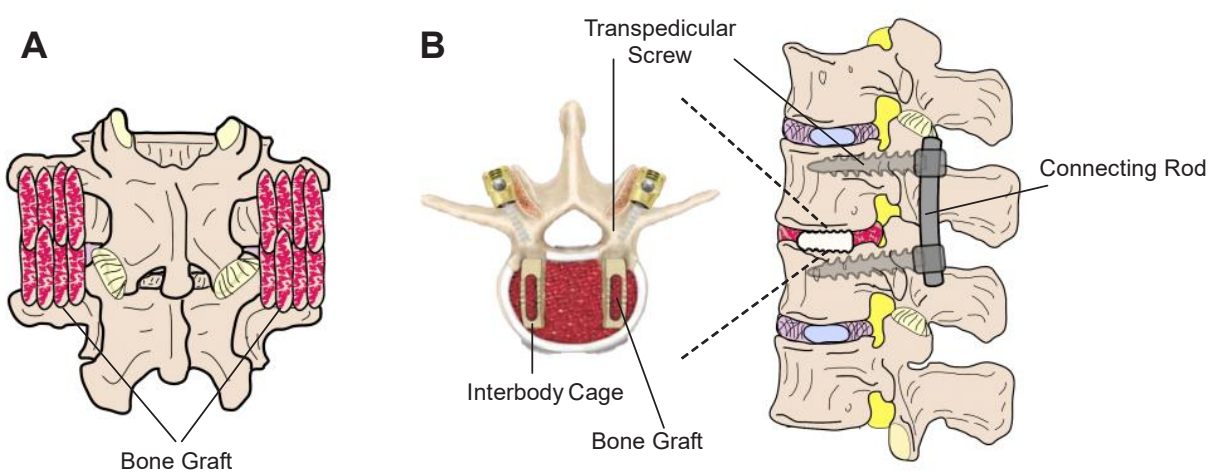

Figure 3. Schematic images of human vertebrae after posterolateral fusion and posterior interbody fusion. A schematic coronal view of posterolateral fusion in which two vertebrae are fused by placing bone graft material over the transverse processes (A). And a schematic axial and sagittal view of the spine after PLIF, showing the cages in white and the bone graft placed inside the cage as well as in the disc space surrounding the cages (B). Transpedicular screws are inserted through the pedicles of the vertebrae and connected by rods to provide initial stability.
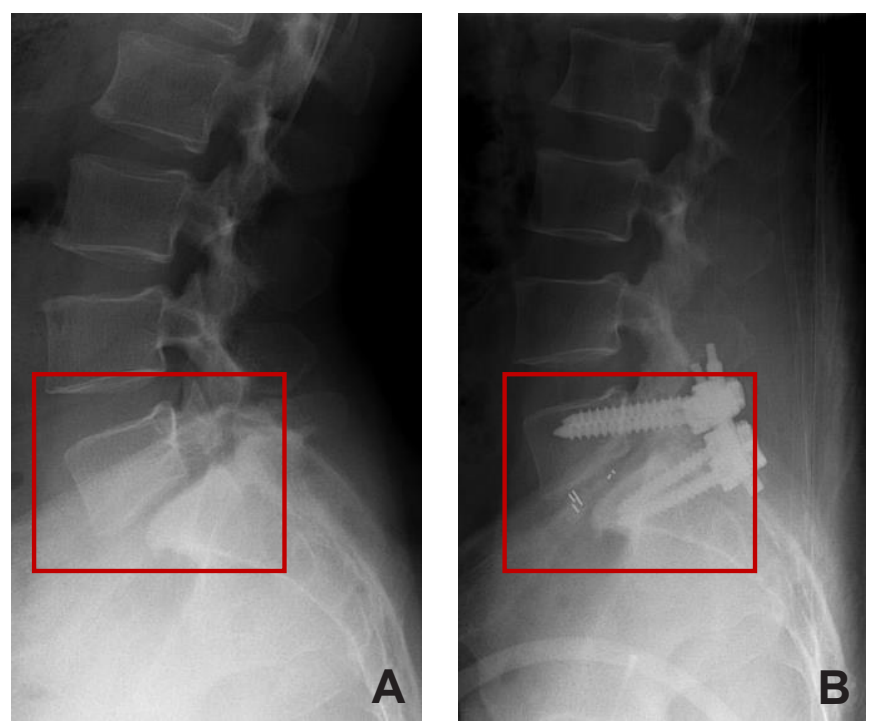

Figure 4. Radiographs of the human spine before and after PLIF, the red boxes delineate the area of surgery. Spondylolisthesis of the lumbar segment L5 - S1 requiring spinal fusion surgery (A). Lumbar segment L5 - S1 after PLIF, the instrumentation and radiopaque markers in the cages are visible (B). 


\section{Bony bridge formation}

To achieve a stable and definite fusion of two or more vertebrae, bony bridges of sufficient quality connecting the vertebrae have to be formed. Osteoblasts are the cells that are responsible for the formation of bone. Osteoblasts originate from mesenchymal stem cells that reside in the bone marrow. These mesenchymal stem cells can differentiate into cells that form mesenchymal tissues such as bone, cartilage, tendon, muscle and ligaments [16]. In response to specific signals from the environment, the mesenchymal stem cells can differentiate into osteoprogenitor cells which can differentiate into osteoblasts. Bone building by osteoblasts is achieved in two steps: the osteoblasts first deposit organic bone matrix which they subsequently mineralize [17]. The organic bone matrix before mineralization is referred to as osteoid [18]. The subsequent mineralization or calcification of the bone matrix relies on the synthesis of hydroxyapatite crystals within the matrix $[17,18]$. After a burst of bone matrix deposition, osteoblasts can either die (apoptosis), become a bone-lining cell, or further differentiate into an osteocyte. Bone-lining cells are inactive flattened osteoblasts that cover the bone surface [19]. Osteoblasts further differentiate into osteocytes when they get trapped in mineralized bone matrix. Osteocytes are spider-shaped cells which form a network for the communication of mechanical and biological signals between cells [17]. Osteocytes can activate osteoblasts but also osteoclasts. Osteoclasts are derived from the monocyte/macrophage lineage [17, 19], responsible for the digestion of cellular waste products. Osteoclasts produce enzymes and act in groups to dissolve mineralized and non-mineralized bone matrix [18]. Remodeling is a continuously ongoing process in all bones in which osteoclasts clean up bone with insufficient quality and osteoblasts rebuild the bone. During life, remodeling continuously maintains bone of sufficient quality and allows the bone to adapt to mechanical changes. The bony bridges between vertebrae undergo remodeling to mature into a fusion mass, capable of weight bearing [20].

\section{Persistent or recurrent pain after PLIF}

The clinical success rate of patients after PLIF is approximately 70\% [21]. A rather large patient group of approximately 30\% suffers from persistent or recurrent pain after PLIF. The diagnosis of a PLIF patient with persistent or recurrent pain includes review of medical history, clinical examination and imaging in order to elucidate the cause of pain. The symptoms of a patient after PLIF can be quantified by means of the same PROMs that were mentioned before to assess symptoms before surgery; VAS, ODI and EQ-5D. Regarding imaging, the same imaging modalities that were used to diagnose the patient with a condition before surgery, can be used to follow-up the patient after surgery (radiography, CT and MRI). 
Radiographs are able to show instrumentation malposition and misalignment of the spinal column. Additionally, when radiographs are made during flexion and extension (flexion-extension radiography), the range of motion within the operated segment can be determined by measuring the angular change between the upper and lower vertebra during bending. However, instrumentation also provides rigidity to the segment that can unfairly be attributed to the bony bridges. Moreover, the absence of motion does not necessarily correspond with solid bony fusion [22-24] and vice versa, the presence of motion is not always related to failed fusion [25-28].

By means of $\mathrm{CT}$, several anatomical abnormalities in a timeframe of months to several years after PLIF can be detected:

- $\quad$ Pseudarthrosis, defined as incomplete fusion [29]. As soon as the bony bridges between the vertebrae become mineralized, which takes about a year to occur, the bony bridges or the absence thereof can be visualized on CT to assess the anatomical success of the surgery.

- Subsidence, which is defined as interbody cage migration or sinking of the cage into the vertebral body [30,31]. Subsidence can be caused by low bone mineral density and can result in a loss of discus height [31].

- $\quad$ Screw loosening or breakage can be caused by vertical stress concentrations at the bone-screw interface or at the base of the pedicle screw respectively, both can be a result of low bone mineral density [32]. Radiolucent lines surrounding the screws on $\mathrm{CT}$ are an indication for screw loosening.

- $\quad$ Adjacent segment disease (degeneration of adjacent intervertebral discs) [33]. As a result of the changes in the operated segment, mechanical alterations in the adjacent segments can occur and cause problems.

In principle, MRI can visualize bony bridging between endplates [34] just as CT, as well as changes in the vertebral body marrow signal as a presumed sign of functional instability $[35,36]$ and disc protrusion or recurrent nerve root compression. However, the use of MRI in PLIF patients is complicated by metal instrumentation which has an effect on the magnets of the scanner [37].

The anatomical result of a spinal fusion procedure (i.e. fusion, pseudarthrosis, subsidence, screw loosening, screw breakage) is important to assess. However, studies in literature have shown that a successful anatomical result after surgery does not automatically guarantee a pain free patient, neither is an anatomical abnormality necessarily associated with symptoms [38-40]. In other words, as was the case for low back pain before surgery, persistent or recurrent pain after PLIF can be multifactorial and non-specific [41, 42], which complicates diagnosis and treatment delineation of the patient. 
Nuclear medicine techniques focus on the imaging of molecular and cellular processes in the human body rather than on the imaging of anatomical structures. Diagnosis of symptomatic patients after spinal fusion can also include such functional modalities, to detect abnormalities in bone metabolism values at specific locations. Nuclear medicine imaging uses the decay characteristics of radioactive molecules to track a certain substance in the human body to provide information on metabolic processes. Different nuclear medicine imaging modalities exist, such as scintigraphy, single photon emission computed tomography (SPECT) and positron emission tomography (PET). By SPECT and PET, three-dimensional images of metabolism can be obtained while scintigraphy provides a two-dimensional image only. Compared to SPECT, PET provides higher resolution, higher sensitivity and better quantification capabilities [43]. Therefore, PET is the nuclear technique that will be evaluated in this thesis.

\section{Positron emission tomography (PET)}

The nucleus of an atom is composed of positively charged protons and neutrons which have no charge. The nucleus is surrounded by a cloud of negatively charged electrons. Radionuclides are nuclei that have an unstable configuration either by an excess number of protons or neutrons and are susceptible to decay in order to change the number of protons or neutrons to obtain a stable configuration by emission of either particles or energy. Positron emission (or $\beta^{+}$decay) is the decay process in which an excess proton is converted into a neutron and a positron, followed by the ejection of the positron from the nucleus which thereby reaches a more stable configuration. Radionuclides that decay via positron emission can be used for PET imaging. Examples are ${ }^{11} \mathrm{C},{ }^{13} \mathrm{~N},{ }^{15} \mathrm{O},{ }^{18} \mathrm{~F},{ }^{22} \mathrm{Na}$ [44]. Each of these radionuclides have their own specific half-life which is defined as the time it takes for half of a certain amount of the radionuclide to decay. The activity of a radionuclide sample at a certain time $(A(t))$ is related to the activity of the sample at time zero $(A(0))$ and the half-life $\left(t_{1 / 2}\right)$ as in (1). The unit of activity is Bequerel $(B q)$. One $B q$ is defined as one atomic disintegration per second.

$$
A(t)=A(0) \cdot e^{t / t_{1 / 2}}
$$

Some radioisotopes are naturally occurring, but radionuclides can also be produced artificially by means of a cyclotron. The cyclotron was invented by Ernest Lawrance in Berkeley, California in 1934 [45]. Radionuclides can directly be used as a tracer for PET imaging or can first be coupled to a biologically active substance, depending on the process of interest in the human body that the tracer should target. The tracer is intravenously injected into the bloodstream of the patient and distributes over the tissues of the body according to its biochemical properties. The positrons that are ejected from the tracer by radioactive decay have a very short lifetime in electron rich material such as human tissue. A positron $\left(\mathrm{e}^{+}\right)$is the antiparticle of an electron $\left(\mathrm{e}^{-}\right)$and as soon as they react, they 
annihilate as in (2); the mass of the electron and the positron is converted into electromagnetic energy which is released in the form of high-energy photons ( $\gamma$ ) (Figure 5A). To conserve momentum and energy, two photons of $511 \mathrm{keV}$ each are emitted simultaneously in exactly opposite directions. The photons generated in this annihilation process are used for imaging in PET.

$$
e^{+}+e^{-} \rightarrow \gamma+\gamma
$$

A PET scanner consists of a ring of detectors surrounding the object that is imaged. When the $511 \mathrm{keV}$ photons fall onto the detectors (Figure 5B), the dense crystalline scintillator material of which the detector is made, emits light. This light is detected by a visible light photon detector which leads to conversion into an electrical current. The location and time of each annihilation (event) are written into a file as raw data. Reconstruction of the raw data (Figure $5 \mathrm{C}$ ) provides a three-dimensional image of the distribution of tracer over the human body (Figure 5D).

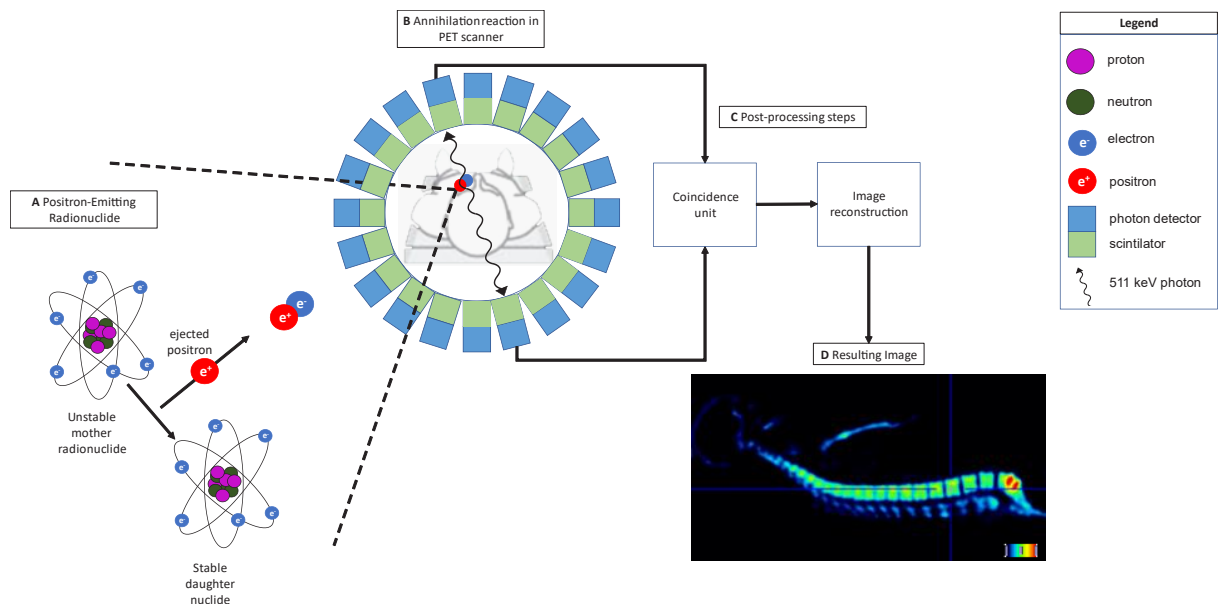

Figure 5. The principle of PET imaging. A positron is emitted from an unstable radionuclide (A) and interacts with an electron through annihilation, the emitted photons are detected by the detectors of the PET scanner (B). A coincidence processing unit reconstructs the raw data (C) to obtain a three-dimensional representation of the tracer distribution in the human body (D). The legend explains the symbols used in the figure.

A major advance in the late 1990s has been the concept of combining molecular imaging with anatomical imaging modalities. Combined PET and CT scanners have been developed that enable coregistered PET and CT images. PET combined with CT allows localization of abnormal uptake which might enhance discriminative power [46]. Furthermore, the CT scan provides tissue density information that allows for attenuation correction of the PET images. 


\section{${ }^{18}$ F-fluoride}

Fluorine-18 $\left({ }_{9}^{18} F\right.$ or $\left.{ }^{18} F\right)$ is a radioactive isotope of fluorine with a half-life of 109.771 minutes. ${ }^{18} \mathrm{~F}$ decays into Oxygen-18 $\left({ }_{8}^{18} \mathrm{O}\right.$ or $\left.{ }^{18} \mathrm{O}\right)$ under emission of a positron $\left(\mathrm{e}^{+}\right)$and a neutrino (ve) as in (3).

$$
{ }_{9}^{18} F \rightarrow{ }_{8}^{18} O+e^{+}+v_{e}
$$

${ }^{18} \mathrm{~F}$ can be introduced into the human body by intravenous injection of ${ }^{18} \mathrm{~F}$ Sodium Fluoride $\left({ }^{18} \mathrm{~F}-\mathrm{NaF}\right)$ and is taken up by bone via ion exchange. The intravenous injected ${ }^{18} \mathrm{~F}$ enters the blood plasma and within minutes travel through the extracellular fluid space into the shell of bound water surrounding the bone crystals [47]. From this point forward, the ions can be considered "localized" in the bone [47]. Within hours, ${ }^{18} \mathrm{~F}$-fluoride will reach the bone crystal surface and within days to weeks ${ }^{18} \mathrm{~F}$ will actually be incorporated into the bone crystal [47]; the fluoride ion exchanges with the hydroxyl group in the unit cell of hydroxyapatite $\left(\mathrm{Ca}_{10}\left(\mathrm{PO}_{4}\right)_{6}(\mathrm{OH})_{2}\right)$ deposited by osteoblasts, to form fluoroapatite $\left(\mathrm{Ca}_{10}\left(\mathrm{PO}_{4}\right)_{6} \mathrm{~F}_{2}\right)$. Fluoride is incorporated during the mineralization phase only, it will not diffuse into already formed bone [48-52]. Not only net deposition of bone mineral, but also areas of osteolytic activity are characterized by an increase of fluoride uptake [47]. The uptake of fluoride is dependent on the available exposed bone crystal surface as well as on the blood flow that delivers the fluoride to the bone crystal $[47,53]$. In other words, processes that increase the exposed bone crystal as well as processes that increase the blood flow are displayed as areas of high fluoride uptake [54]. The ${ }^{18}$ F-fluoride PET technique has been validated by comparison with bone histomorphometry [51, 55-57], as well as by comparison with biochemical markers of bone turnover [58].

\section{${ }^{18}$ F-fluoride PET/CT analysis methods}

Analysis and interpretation of the ${ }^{18} \mathrm{~F}$-fluoride PET/CT scans can be done visually. In addition, a semi-quantitative measure called the Standardized Uptake Value (SUV) can be calculated for specific regions of interest (ROIs). To calculate SUV, the uptake of fluoride in a certain ROI (activity, A) is corrected for the dose of radioactive tracer that has been injected (injected dose, ID) and for the mass of the patient (mass, $m$ ), see (4).

$$
S U V=\frac{A}{I D / m}
$$

Another analysis method to obtain quantitative measures for bone metabolism involves pharmacokinetic modeling of the uptake process of fluoride, from extraction from the blood plasma to incorporation in the skeletal system. The model as described by Hawkins et al. consists of three compartments: a vascular compartment in which the fluoride ion is present in the blood plasma, an extravascular bone compartment in which the fluoride ion is present in free form and a compartment of bound fluoride in which the fluoride is 
attached to bone either on the surface or incorporated into the hydroxyapatite crystal [59] (Figure 6).

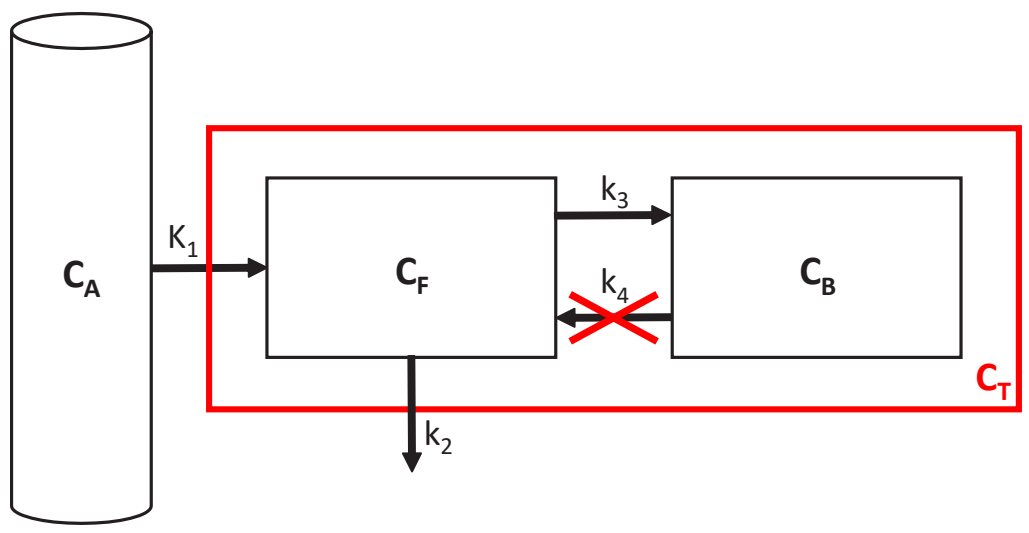

Figure 6. The three-compartment model for fluoride bone metabolism. Each compartment contains a certain concentration of ${ }^{18} \mathrm{~F}$-fluoride: $\mathrm{C}_{\mathrm{A}}[\mathrm{Bq} / \mathrm{ml}]$ is the arterial radioactivity concentration in the blood plasma compartment, $\mathrm{C}_{\mathrm{F}}[\mathrm{Bq} / \mathrm{ml}]$ represents the free concentration in the extravascular space compartment, unbound to bone and $\mathrm{C}_{\mathrm{B}}[\mathrm{Bq} / \mathrm{ml}]$ refers to the radioactivity bound to bone either on the bone surface or fully incorporated in the hydroxyapatite. $\mathrm{C}_{\mathrm{T}}[\mathrm{Bq} / \mathrm{ml}]$ represents the total tissue radioactivity concentration that the PET scanner will measure. The rate constants $K_{1}, k_{2}, k_{3}$ and $k_{4}$ describe the transport rate of the fluoride between the compartments. Rate constant $k_{4}$ can be considered negligible.

Rate constants between the compartments represent the speed with which the fluoride travels from one compartment to the next. $K_{1}$ and $k_{2}$ represent the forward and reverse transport of fluoride from plasma to the tissue compartments [59]. $k_{3}$ and $k_{4}$ represent the incorporation and release of fluoride from the bound compartment [59]. As the fluoride that binds to bone is not expected to be subsequently released within the measuring time, $k_{4}$ is set to zero. The values of these rate constants can be combined into physiologically meaningful parameters related to regional bone metabolism $[55,59,60]$ which correlate to histomorphometric bone formation parameters [55, 61]. Additionally, parameters related to bone blood flow [62] and bone mineral incorporation [63] can be calculated.

For pharmacokinetic modeling, the path of the tracer from the blood to the bone should be recorded which means that from the moment of tracer injection onwards, the location of the tracer should be measured. This is referred to as dynamic scanning. For visual analysis of the PET/CT images as well as for semi-quantification by the calculation of SUV, a static PET/CT measurement suffices. A schematic representation of a PET/CT acquisition including a 30-minute dynamic scan from the moment of tracer injection onwards and a static scan at 60 minutes after tracer injection is shown in Figure 7. 


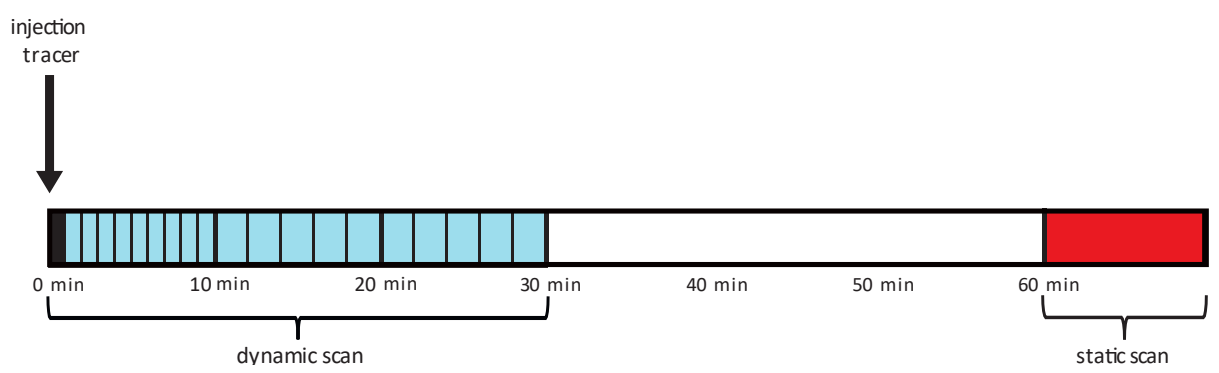

Figure 7. A schematic representation of a PET/CT acquisition including a 30-minute dynamic scan and a static scan. The tracer is injected at time point 0 minutes. At the same time as injection of the tracer, the dynamic scan (in blue) starts and lasts for 30 minutes with time frames of 5 seconds to 120 seconds as indicated by the vertical lines. At 60 minutes after injection of the tracer, a static scan is made (in red).

All in all, imaging plays an important role in setting the diagnosis in patients with persistent or recurrent pain after spinal fusion to be able to delineate the most beneficial line of treatment for a specific patient. In this thesis we hypothesize that findings on the functional imaging modality PET/CT are more related to a patient's clinical status and thus a useful addition in the follow-up of patients with persisting or recurrent symptoms after spinal fusion. Furthermore, physiological processes can be visualized with PET before manifestation of morphological changes, and therefore we hypothesize that PET can also be of value in PLIF patients in the early postoperative phase. 


\section{OBJECTIVES AND THESIS OUTLINE}

The objective of this thesis was to evaluate the diagnostic potential of ${ }^{18} \mathrm{~F}$-fluoride PET/CT scanning in the follow-up of patients after posterior lumbar interbody fusion (PLIF).

The specific objectives of this thesis were:

To provide an overview of the diagnostic accuracy of imaging modalities to detect pseudarthrosis after spinal fusion, with surgical exploration as reference standard

Follow-up of patients after spinal fusion to verify whether bony fusion has been achieved or whether pseudarthrosis has developed, can be performed with a number of imaging modalities. Chapter 2 summarized studies that investigated the diagnostic accuracy of clinically used imaging modalities to detect pseudarthrosis after thoracolumbar spinal fusion in a systematic review. Studies that compared the accuracy of a modality for the diagnosis of pseudarthrosis to the gold standard of surgical exploration were included. Subgroup meta-analysis was performed for each modality that was described by two or more comparable studies, to obtain a single outcome measure of diagnostic accuracy for each imaging modality.

To investigate the relationship between ${ }^{18} \mathrm{~F}$-fluoride PET/CT, CT and clinical status several years after PLIF in symptomatic patients and asymptomatic patients

In case a patient suffers from persistent or recurrent symptoms after PLIF, it is important to localize the pain generator to be able to delineate the most beneficial treatment strategy for a specific patient. Imaging plays an important role in setting the diagnosis. However, the anatomical result of a spinal fusion procedure (i.e. fusion, pseudarthrosis, subsidence, instrumentation failure etc.), is not necessarily related to the clinical status of a patient. As ${ }^{18} \mathrm{~F}$-fluoride PET/CT is able to visualize bone metabolic activity, it could pinpoint spots with irregular bone metabolism that might correspond to the symptoms.

In Chapter 3 and Chapter 4, patients one year or more after PLIF were evaluated both anatomically on CT and functionally by means of ${ }^{18} \mathrm{~F}$-fluoride PET/CT. To identify abnormalities that could be potential pain generators, the PET and CT parameters were related to the clinical status of the patients as reported by patient reported outcome measures (PROMs) in symptomatic patients and asymptomatic patients. Chapter 3 focused on the endplates and the bone graft in the intervertebral disc space that are directly involved in the spinal fusion procedure. Chapter 4 also evaluated the facet joints, screws and adjacent levels that might indirectly be involved, as biomechanical changes may occur in the adjacent segments after immobilization of a segment. 
To develop an analysis method for dynamic ${ }^{18}$ F-fluoride PET/CT scans of patients after PLIF

CT allows visualization and quantification of callus formation and bony bridging between the involved vertebrae. However, since these morphological signs of fusion occur late in the normal course of bone healing, CT is of limited use in the early postoperative phase. $\mathrm{PET} / \mathrm{CT}$ may prove valuable in the follow-up of spinal fusion patients early after surgery as changes in bone metabolism can precede morphological alterations. Especially pharmacokinetic modeling can be of value since this yields parameters related to bone blood flow and exposed bone crystal surface; both processes that occur early in the normal course of bone healing.

Before pharmacokinetic modeling was applied to a prospective cohort of PLIF patients early after surgery, an analysis method was developed in a retrospective cohort of PLIF patients. The distribution of tracer, following intravenous injection, from the aorta to the bone can be visualized and quantified by means of dynamic scanning. Dynamic analysis requires an input function. The input function can either be obtained via blood sampling, or by an image derived input function. Chapter 5 proposed an analysis method for pharmacokinetic modeling of dynamic ${ }^{18} \mathrm{~F}$-fluoride PET/CT scans in patients after PLIF using an image derived input function. Outcome parameters from dynamic scanning were compared to the parameters of static scanning.

To investigate the potential of $a^{18}$ F-fluoride PET/CT scan early after PLIF to predict fusion outcome at one year

In Chapter 6, twenty prospectively included PLIF patients underwent ${ }^{18} \mathrm{~F}$-fluoride PET/CT scanning at six weeks and at one year after surgery. A diagnostic CT scan at one year was used to assess whether the included patients attained solid fusion or suffered from pseudarthrosis. The analysis method developed in Chapter 5 was used to obtain dynamic parameters as well as static parameters. PET parameters at six weeks were compared to the fusion status at one year to determine whether PET/CT early after surgery can predict bony fusion.

A general discussion elaborating on the interpretation of ${ }^{18} \mathrm{~F}$-fluoride PET/CT results for clinical practice in PLIF patients, other possible applications and recommendations for future research were provided in Chapter 7. 


\section{REFERENCES}

1. Global, regional, and national incidence, prevalence, and years lived with disability for 301 acute and chronic diseases and injuries in 188 countries, 1990-2013: a systematic analysis for the Global Burden of Disease Study 2013. Lancet (London, England). 2015; 386(9995):743-800.

2. Hoy D, March L, Brooks P, Blyth F, Woolf A, Bain C, et al. The global burden of low back pain: estimates from the Global Burden of Disease 2010 study. Annals of the rheumatic diseases. 2014; 73(6):968-974.

3. Hartvigsen J, Hancock MJ, Kongsted A, Louw Q, Ferreira ML, Genevay S, et al. What low back pain is and why we need to pay attention. Lancet (London, England). 2018; 391(10137):2356-2367.

4. Burke JG, Watson RW, McCormack D, Dowling FE, Walsh MG, Fitzpatrick JM. Intervertebral discs which cause low back pain secrete high levels of proinflammatory mediators. The Journal of bone and joint surgery British volume. 2002; 84(2):196-201.

5. Kang JD, Stefanovic-Racic M, McIntyre LA, Georgescu HI, Evans CH. Toward a biochemical understanding of human intervertebral disc degeneration and herniation. Contributions of nitric oxide, interleukins, prostaglandin E2, and matrix metalloproteinases. Spine. 1997; 22(10):1065-1073.

6. https://sites.google.com/a/wisc.edu/neuroradiology/anatomy/spine/slide-2---imaging-the-spine.

7. Zanoli G, Stromqvist B, Jonsson B. Visual analog scales for interpretation of back and leg pain intensity in patients operated for degenerative lumbar spine disorders. Spine. 2001; 26(21):2375-2380.

8. Fairbank JC, Pynsent PB. The Oswestry Disability Index. Spine. 2000; 25(22):2940-2952; discussion 2952.

9. Fairbank JC, Couper J, Davies JB, O'Brien JP. The Oswestry low back pain disability questionnaire. Physiotherapy. 1980; 66(8):271-273.

10. Lamers LM, Stalmeier PF, McDonnell J, Krabbe PF, van Busschbach JJ. [Measuring the quality of life in economic evaluations: the Dutch EQ-5D tariff]. Nederlands tijdschrift voor geneeskunde. 2005; 149(28):1574-1578.

11. Lamers LM, McDonnell J, Stalmeier PF, Krabbe PF, Busschbach JJ. The Dutch tariff: results and arguments for an effective design for national EQ-5D valuation studies. Health economics. 2006; 15(10):1121-1132.

12. Albee FH. Transplantation of a portion of the tibia into the spine for Pott's disease: a preliminary report. Journal of the American Medical Association. 1911; 57(11):885-886.

13. Hibbs RA. An operation for progressive spinal deformities. NY Med J. 1911; 93:1013.

14. Mobbs RJ, Phan K, Malham G, Seex K, Rao PJ. Lumbar interbody fusion: techniques, indications and comparison of interbody fusion options including PLIF, TLIF, MI-TLIF, OLIF/ATP, LLIF and ALIF. Journal of spine surgery (Hong Kong). 2015; 1(1):2-18.

15. Rajaee SS, Bae HW, Kanim LE, Delamarter RB. Spinal fusion in the United States: analysis of trends from 1998 to 2008. Spine. 2012; 37(1):67-76.

16. Bruder SP, Fink DJ, Caplan AI. Mesenchymal stem cells in bone development, bone repair, and skeletal regeneration therapy. Journal of cellular biochemistry. 1994; 56(3):283-294.

17. Capulli M, Paone R, Rucci N. Osteoblast and osteocyte: games without frontiers. Archives of biochemistry and biophysics. 2014; 561:3-12.

18. Pilitsis JG, Lucas DR, Rengachary SS. Bone healing and spinal fusion. Neurosurgical focus. 2002; $13(6)$ :e1.

19. Feng X, Teitelbaum SL. Osteoclasts: New Insights. Bone research. 2013; 1(1):11-26. 
20. Ludwig SC, Boden SD. Osteoinductive bone graft substitutes for spinal fusion: a basic science summary. The Orthopedic clinics of North America. 1999; 30(4):635-645.

21. Makanji H, Schoenfeld AJ, Bhalla A, Bono CM. Critical analysis of trends in lumbar fusion for degenerative disorders revisited: influence of technique on fusion rate and clinical outcomes. European spine journal : official publication of the European Spine Society, the European Spinal Deformity Society, and the European Section of the Cervical Spine Research Society. 2018; 27(8):1868-1876.

22. Blumenthal SL, Gill K. Can lumbar spine radiographs accurately determine fusion in postoperative patients? Correlation of routine radiographs with a second surgical look at lumbar fusions. Spine. 1993; 18(9):1186-1189.

23. Kant AP, Daum WJ, Dean SM, Uchida T. Evaluation of lumbar spine fusion. Plain radiographs versus direct surgical exploration and observation. Spine. 1995; 20(21):2313-2317.

24. Larsen JM, Capen DA. Pseudarthrosis of the Lumbar Spine. The Journal of the American Academy of Orthopaedic Surgeons. 1997; 5(3):153-162.

25. Choudhri TF, Mummaneni PV, Dhall SS, Eck JC, Groff MW, Ghogawala Z, et al. Guideline update for the performance of fusion procedures for degenerative disease of the lumbar spine. Part 4: radiographic assessment of fusion status. Journal of neurosurgery Spine. 2014; 21(1):23-30.

26. McAfee PC, Boden SD, Brantigan JW, Fraser RD, Kuslich SD, Oxland TR, et al. Symposium: a critical discrepancy-a criteria of successful arthrodesis following interbody spinal fusions. Spine. 2001; 26(3):320-334.

27. Santos ER, Goss DG, Morcom RK, Fraser RD. Radiologic assessment of interbody fusion using carbon fiber cages. Spine. 2003; 28(10):997-1001.

28. Frymoyer JW, Hanley EN, Jr., Howe J, Kuhlmann D, Matteri RE. A comparison of radiographic findings in fusion and nonfusion patients ten or more years following lumbar disc surgery. Spine. 1979; 4(5):435-440.

29. Chun DS, Baker KC, Hsu WK. Lumbar pseudarthrosis: a review of current diagnosis and treatment. Neurosurgical focus. 2015; 39(4):E10.

30. Lee DY, Park YJ, Song SY, Jeong ST, Kim DH. Risk Factors for Posterior Cage Migration after Lumbar Interbody Fusion Surgery. Asian spine journal. 2018; 12(1):59-68.

31. Oh KW, Lee JH, Lee JH, Lee DY, Shim HJ. The Correlation Between Cage Subsidence, Bone Mineral Density, and Clinical Results in Posterior Lumbar Interbody Fusion. Clinical spine surgery. 2017; 30(6):E683-E689.

32. Okuyama K, Abe E, Suzuki T, Tamura Y, Chiba M, Sato K. Can insertional torque predict screw loosening and related failures? An in vivo study of pedicle screw fixation augmenting posterior lumbar interbody fusion. Spine. 2000; 25(7):858-864.

33. Okuda S, Nagamoto Y, Matsumoto T, Sugiura T, Takahashi Y, Iwasaki M. Adjacent Segment Disease after Single Segment Posterior Lumbar Interbody Fusion for Degenerative Spondylolisthesis: Minimum 10 Years Follow Up. Spine. 2018.

34. Kroner AH, Eyb R, Lange A, Lomoschitz K, Mahdi T, Engel A. Magnetic resonance imaging evaluation of posterior lumbar interbody fusion. Spine. 2006; 31(12):1365-1371.

35. Lang P, Chafetz N, Genant HK, Morris JM. Lumbar spinal fusion. Assessment of functional stability with magnetic resonance imaging. Spine. 1990; 15(6):581-588.

36. Steinmann JC, Herkowitz HN. Pseudarthrosis of the spine. Clinical orthopaedics and related research. 1992(284):80-90.

37. Williams AL, Gornet MF, Burkus JK. CT evaluation of lumbar interbody fusion: current concepts. AJNR American journal of neuroradiology. 2005; 26(8):2057-2066. 
38. Heggeness MH, Esses SI. Classification of pseudarthroses of the lumbar spine. Spine. 1991; 16(8 Suppl):S449-454.

39. Raizman NM, O'Brien JR, Poehling-Monaghan KL, Yu WD. Pseudarthrosis of the spine. The Journal of the American Academy of Orthopaedic Surgeons. 2009; 17(8):494-503.

40. Rothman RH, Booth R. Failures of spinal fusion. The Orthopedic clinics of North America. 1975; 6(1):299304.

41. Heggeness MH, Esses SI, Mody DR. A histologic study of lumbar pseudarthrosis. Spine. 1993; 18(8):10161020.

42. Larsen JM, Rimoldi RL, Capen DA, Nelson RW, Nagelberg S, Thomas JC, Jr. Assessment of pseudarthrosis in pedicle screw fusion: a prospective study comparing plain radiographs, flexion/extension radiographs, CT scanning, and bone scintigraphy with operative findings. Journal of spinal disorders. 1996; 9(2):117120.

43. Rahmim A, Zaidi H. PET versus SPECT: strengths, limitations and challenges. Nuclear medicine communications. 2008; 29(3):193-207.

44. Cherry SR, Dahlbom M. PET: physics, instrumentation, and scanners. PET; 2006: Springer; 2006. p. 1-117.

45. AGENCY IAE. Cyclotron Produced Radionuclides: Physical Characteristics and Production Methods. Vienna: INTERNATIONAL ATOMIC ENERGY AGENCY, 2009.

46. Rager O, Schaller K, Payer M, Tchernin D, Ratib O, Tessitore E. SPECT/CT in differentiation of pseudarthrosis from other causes of back pain in lumbar spinal fusion: report on 10 consecutive cases. Clinical nuclear medicine. 2012; 37(4):339-343.

47. Blau M, Ganatra R, Bender MA. 18 F-fluoride for bone imaging. Seminars in nuclear medicine. 1972; 2(1):31-37.

48. Anderson HC. Matrix vesicles and calcification. Current rheumatology reports. 2003; 5(3):222-226.

49. Chachra D, Vieira AP, Grynpas MD. Fluoride and mineralized tissues. Critical reviews in biomedical engineering. 2008; 36(2-3):183-223.

50. Grynpas MD. Fluoride effects on bone crystals. Journal of bone and mineral research : the official journal of the American Society for Bone and Mineral Research. 1990; 5 Suppl 1:S169-175.

51. Toegel S, Hoffmann O, Wadsak W, Ettlinger D, Mien LK, Wiesner K, et al. Uptake of bone-seekers is solely associated with mineralisation! A study with 99mTc-MDP, 153Sm-EDTMP and 18F-fluoride on osteoblasts. European journal of nuclear medicine and molecular imaging. 2006; 33(4):491-494.

52. Zipkin I, Mc CF. Deposition of fluorine in the bones and teeth of the growing rat. The Journal of nutrition. 1952; 47(4):611-620.

53. Segall G, Delbeke D, Stabin MG, Even-Sapir E, Fair J, Sajdak R, et al. SNM practice guideline for sodium 18Ffluoride PET/CT bone scans 1.0. Journal of nuclear medicine : official publication, Society of Nuclear Medicine. 2010; 51(11):1813-1820.

54. Foldager CB, Bendtsen M, Bunger C. PET scanning for evaluation of bone metabolism. Acta orthopaedica. 2009; 80(6):737-738; author reply 738-739.

55. Messa C, Goodman WG, Hoh CK, Choi Y, Nissenson AR, Salusky IB, et al. Bone metabolic activity measured with positron emission tomography and [18F]fluoride ion in renal osteodystrophy: correlation with bone histomorphometry. The Journal of clinical endocrinology and metabolism. 1993; 77(4):949-955.

56. Anderson HC. Matrix vesicles and calcification. Current rheumatology reports. 2003; 5(3):222-226. 
57. Piert M, Park H, Khan A, Siddiqui J, Hussain $\mathrm{H}$, Chenevert $\mathrm{T}$, et al. Detection of aggressive primary prostate cancer with 11C-choline PET/CT using multimodality fusion techniques. Journal of nuclear medicine : official publication, Society of Nuclear Medicine. 2009; 50(10):1585-1593.

58. Frost ML, Blake GM, Park-Holohan SJ, Cook GJ, Curran KM, Marsden PK, et al. Long-term precision of 18Ffluoride PET skeletal kinetic studies in the assessment of bone metabolism. Journal of nuclear medicine : official publication, Society of Nuclear Medicine. 2008; 49(5):700-707.

59. Hawkins RA, Choi Y, Huang SC, Hoh CK, Dahlbom M, Schiepers C, et al. Evaluation of the skeletal kinetics of fluorine-18-fluoride ion with PET. Journal of nuclear medicine : official publication, Society of Nuclear Medicine. 1992; 33(5):633-642.

60. Piert M, Zittel TT, Becker GA, Jahn M, Stahlschmidt A, Maier G, et al. Assessment of porcine bone metabolism by dynamic. Journal of nuclear medicine : official publication, Society of Nuclear Medicine. 2001; 42(7):1091-1100.

61. Piert M, Zittel TT, Machulla HJ, Becker GA, Jahn M, Maier G, et al. Blood flow measurements with [(15)O]H2O and [18F]fluoride ion PET in porcine vertebrae. Journal of bone and mineral research : the official journal of the American Society for Bone and Mineral Research. 1998; 13(8):1328-1336.

62. Puri T, Frost ML, Curran KM, Siddique M, Moore AE, Cook GJ, et al. Differences in regional bone metabolism at the spine and hip: a quantitative study using (18)F-fluoride positron emission tomography. Osteoporosis international : a journal established as result of cooperation between the European Foundation for Osteoporosis and the National Osteoporosis Foundation of the USA. 2013; 24(2):633-639.

63. Frost ML, Blake GM, Cook GJ, Marsden PK, Fogelman I. Differences in regional bone perfusion and turnover between lumbar spine and distal humerus: (18)F-fluoride PET study of treatment-naive and treated postmenopausal women. Bone. 2009; 45(5):942-948. 


\section{CHAPTER 2}

The diagnostic accuracy of imaging modalities to detect pseudarthrosis after spinal fusion a systematic review and meta-analysis of the literature

Marloes JM Peters, Carolien HG Bastiaenen, Boudewijn T Brans, René E Weijers, Paul C Willems 


\section{ABSTRACT}

\section{OBJECTIVES}

The aim of the study was to determine the diagnostic accuracy of imaging modalities to detect pseudarthrosis after thoracolumbar spinal fusion, with surgical exploration as reference standard.

\section{METHODS}

A systematic literature search for original studies was performed on the diagnostic accuracy of imaging as index test compared to surgical exploration as reference standard to diagnose pseudarthrosis after thoracolumbar spinal fusion. Diagnostic accuracy values were extracted and methodologic quality of studies was evaluated by the Quality Assessment of Diagnostic Accuracy Studies 2 (QUADAS-2) tool. Per modality, clinically comparable studies were included in subgroup meta-analysis and weighted odds ratio's (OR's) were calculated using the random effect model.

\section{RESULTS}

15 studies were included. Risk of bias was classified as high/unclear in 58\% of the studies. Concerns of applicability was classified as high/unclear in $40 \%$ of the studies. Four scintigraphy studies including 93 patients in total, were pooled to OR=2.91 (95\% confidence interval [Cl]: 0.93-9.13). Five studies on plain radiography with 398 patients in total, were pooled into $\mathrm{OR}=7.07$ (95\% Cl: 2.97-16.86). Two studies evaluating flexion-extension radiography of 75 patients in total were pooled into $\mathrm{OR}=4.00$ ( $95 \% \mathrm{Cl}: 0.15-105.96)$. Two studies of 68 patients in total were pooled for $\mathrm{CT}$ and yielded $\mathrm{OR}=17.02$ ( $95 \% \mathrm{Cl}$ : 6.42-45.10). A single study reporting on polytomography, $\mathrm{OR}=10.15(95 \% \mathrm{Cl} 5.49-18.78)$ was also considered to be an accurate study.

\section{CONCLUSION}

With a pooled OR of 17.02, CT can be considered the most accurate imaging modality for the detection of pseudarthrosis after thoracolumbar spinal fusion from this review. 


\title{
INTRODUCTION
}

Low back pain is a global health and socio-economic problem [1] as it is the leading cause of disability and work-absenteeism in the western world [2]. When conservative measures fail, operative intervention can be considered. Spinal fusion is a surgical procedure in which rigid fixation of vertebral segments is achieved by means of osteosynthesis and bone grafting to create definite bony fusion of the vertebrae involved. Failed spinal fusion may occur in $30-40 \%$ of spinal fusion patients [3,4]. Pseudarthrosis is defined as the absence of solid bony fusion at a minimum follow-up of six months after surgery [5, 6]. Pseudarthrosis can be associated with persistent or recurrent back and/or leg pain [7], but can also be asymptomatic [7-9]. Whether symptomatic or asymptomatic, pseudarthrosis increases the risk of material failure, late deformity and neurological symptoms $[10,11]$.

Revision surgery is the preferred treatment in spinal fusion patients suffering from symptoms due to pseudarthrosis. Revision surgery is invasive, expensive and may have a worse outcome than primary surgery $[12,13]$ and should only be performed when the pseudarthrosis diagnosis is irrefutable. Since symptoms of pseudarthrosis may be nonspecific and multiple individual sources of pain may contribute to the complex of symptoms [14], diagnostic tools are required to set the diagnosis. The gold standard for the diagnosis of pseudarthrosis is surgical exploration $[5,7,15,16]$, an invasive, costly and nowadays rarely used test which is not desirable or ethical in patients without symptoms. The aim of the study was to determine the diagnostic accuracy of imaging modalities to detect pseudarthrosis after thoracolumbar spinal fusion, with surgical exploration as reference standard.

\section{MATERIALS AND METHODS}

\author{
Identification of studies
}

This review was performed according to the PRISMA statement guidelines [17, 18]. A systematic literature search was conducted in the PubMed, EMBASE and CINAHL databases from inception until February 2017 to identify relevant studies. A list of keywords and text words was formulated to describe the detection of pseudarthrosis by imaging as index test compared to surgical exploration as reference standard in patients after spinal fusion surgery. Terms for imaging: tomography, radiography, plain radiographs, MRI, CT, scintigraphy, SPECT, SPECT/CT, PET, PET/CT, DEXA. Terms for study design: diagnostic accuracy, precision, predictive value, sensitivity, specificity, false positive, false negative. Terms for patient population: spine, vertebrae, vertebral column, spinal fusion, spinal arthrodesis, spondylodesis, bone graft, pseudarthrosis, non-union, delayed union, clinical 
failure, surgical exploration, re-operation, second-look operation. The search was limited to the English language.

Once the search was completed, the resulting articles were checked for duplicates. Subsequently, two independent reviewers (PW, orthopedic surgeon with over 10 years of experience in spinal surgery and MP, junior researcher specialized in imaging) screened the identified citations to determine whether they met predefined in- and exclusion criteria. If disagreements could not be resolved by consensus, a third reviewer (CB, clinical epidemiologist with years of experience in conducting systematic reviews) was consulted. Only original studies that provided data to construct contingency tables, were included. Exclusion criteria were spinal fusion for the indications bone fracture, tumor, infection; time interval between surgery and index test less than 6 months; patient population smaller than 10; cervical fusion; animal studies; in vitro studies.

\section{Data extraction}

Standard reference data, population characteristics, details on spinal fusion, index test, reference test and time intervals were extracted by the reviewers (PW, MP). Disagreements were resolved by consensus. Besides study characteristics, diagnostic accuracy data was extracted. Since the outcome was dichotomous (diagnosis was either pseudarthrosis or fusion), contingency tables were constructed. Also, we recorded whether the results originated from per-patient-, per-level- or per-side-based analysis.

\section{Methodological quality}

The methodological quality of each selected study was assessed independently by the reviewers according to the Quality Assessment for Diagnostic Accuracy Studies 2 (QUADAS-2) tool [19]. The QUADAS-2 tool consists of four key domains that discuss patient selection, index test, reference standard, flow of patients through the study and timing of the index test and reference standard. Each domain was scored in terms of risk of bias and concerns regarding applicability to the research question. Disagreements were resolved by consensus.

\section{Data synthesis and statistical analysis}

Pseudarthrosis was defined as a positive test result and fusion as a negative test result. Diagnostic accuracy values were calculated from the extracted contingency tables. Continuity correction was applied to studies with zero-cell counts by adding 0.5 to all cells of the study [20]. Per index test, the studies describing that test were considered for inclusion into subgroup meta-analysis. 
Inclusion in meta-analysis

Meta-analysis was only performed when studies evaluating the same modality were not significantly hampered by clinical heterogeneity. Studies were considered clinically heterogeneous when patient groups, outcome measures and/or the execution of index tests were considerably different.

The random effect model was employed during meta-analyses to account for unobserved sources of variation [21]. The odds ratio (OR) was used as the principal summary measure in meta-analysis. The higher the OR, the better the discriminatory performance. An OR of 1 indicates a test that does not discriminate between patients with pseudarthrosis and patients with fusion [22]. An OR below 1 suggests a negative association between index test and surgical exploration. Analyses were performed using the Stata statistical software package, version 14.1 (Statacorp, College Station, Texas, USA).

\section{RESULTS}

Identification of studies

165 potentially relevant references were identified through database search. After screening, 15 studies were included in this review, reporting on 8 modalities; plain radiography, flexion extension radiography (FE radiography), computed tomography (CT), single photon emission computed tomography (SPECT), planar scintigraphy, polytomography, ultra sound/sonography (US) and ${ }^{18} \mathrm{~F}$-fluoride positron emission tomography/computed tomography (PET/CT). The study selection flowchart is detailed in Figure 1 . The level of evidence of the included studies ranged from I to III.

\section{Data extraction}

Study characteristics of the 15 included studies are listed in Table 1. The number of levels fused in a single patient during initial surgery ranged from 1 to 13 levels. Eight articles monitored pseudarthrosis per patient, five monitored each level separately and two made a distinction between the left and right side of each operated level. All articles reported that persistent low back pain and/or suspicion of pseudarthrosis was the reason for surgical exploration. The time interval between initial surgery and surgical exploration ranged from 6 to 120 months. 


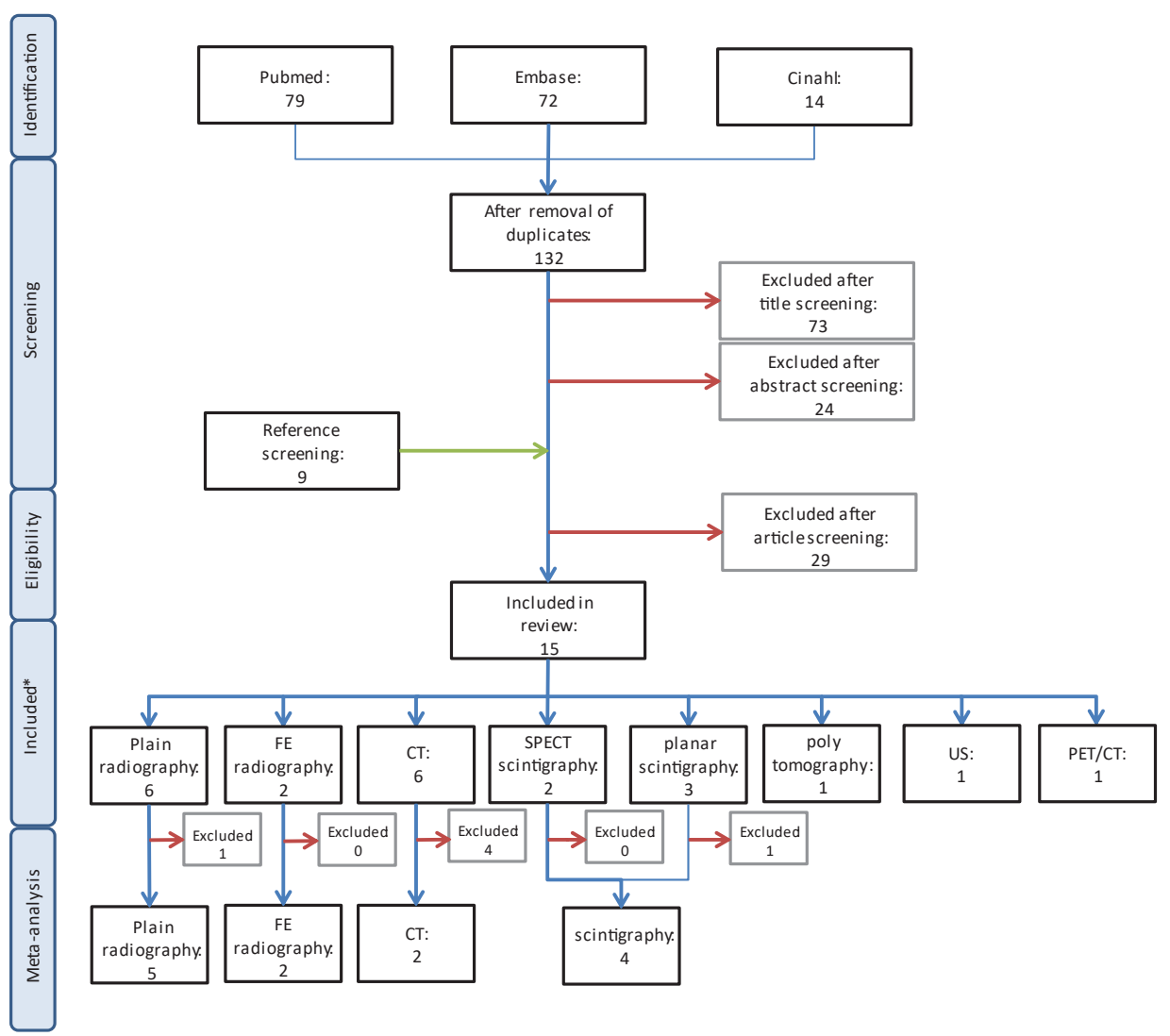

Figure 1. Flowchart showing the selection of studies from electronic search (identification) until inclusion in the subgroup meta-analyses. Initially, 165 potentially relevant references were identified through database search. 132 were obtained for further screening after removal of 33 duplicates. After removal based on title and abstract screening, the full text of 35 articles was screened and their reference sections were scanned for additional eligible studies. Hereafter, 15 studies were included this review, reporting on 8 modalities. The metaanalysis part at the bottom of the figure, will be discussed in 'inclusion in meta-analysis', which can be found hereafter in the result section. ${ }^{*} 3$ of the 15 studies described 2 to 4 modalities, leading to 22 included items. 


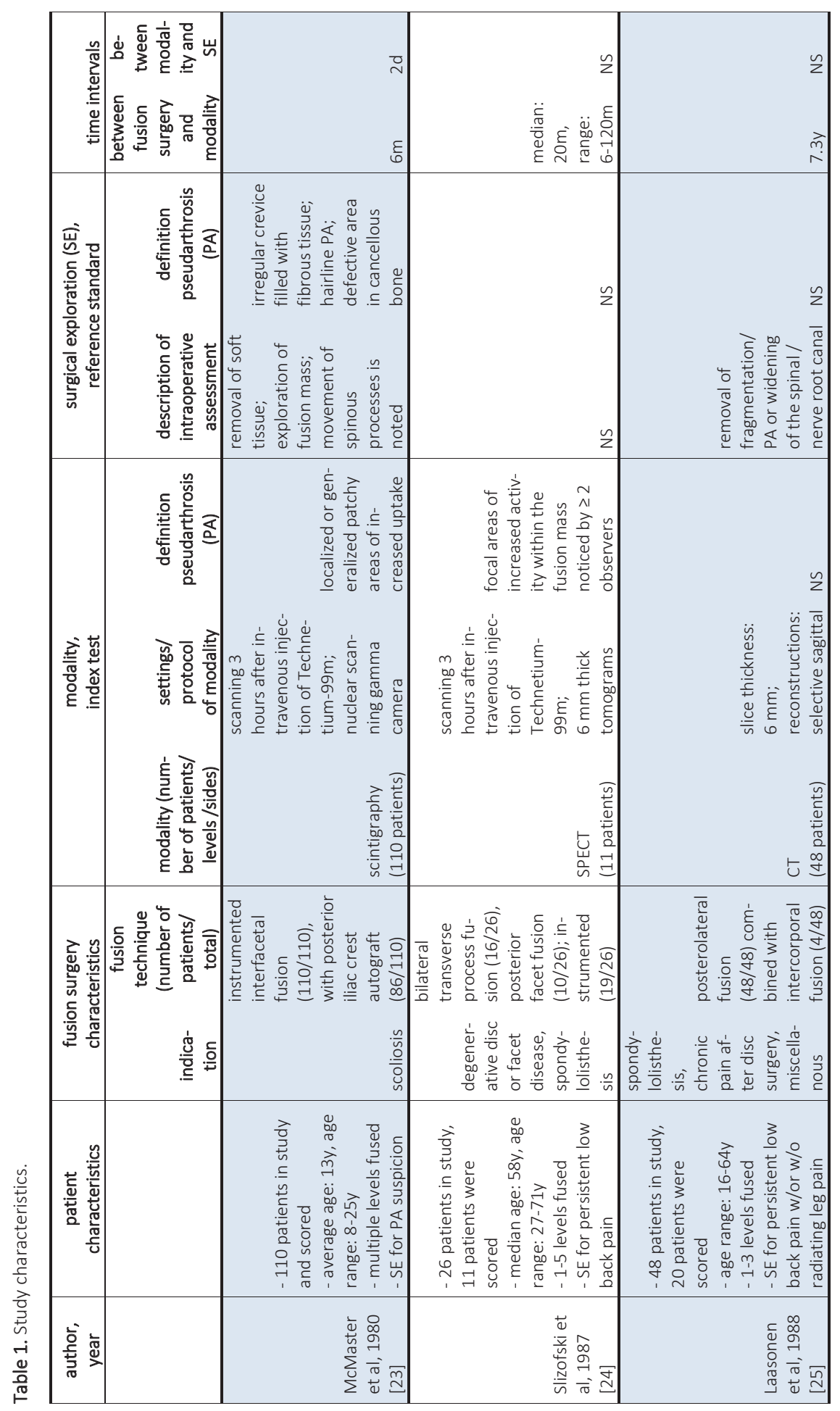




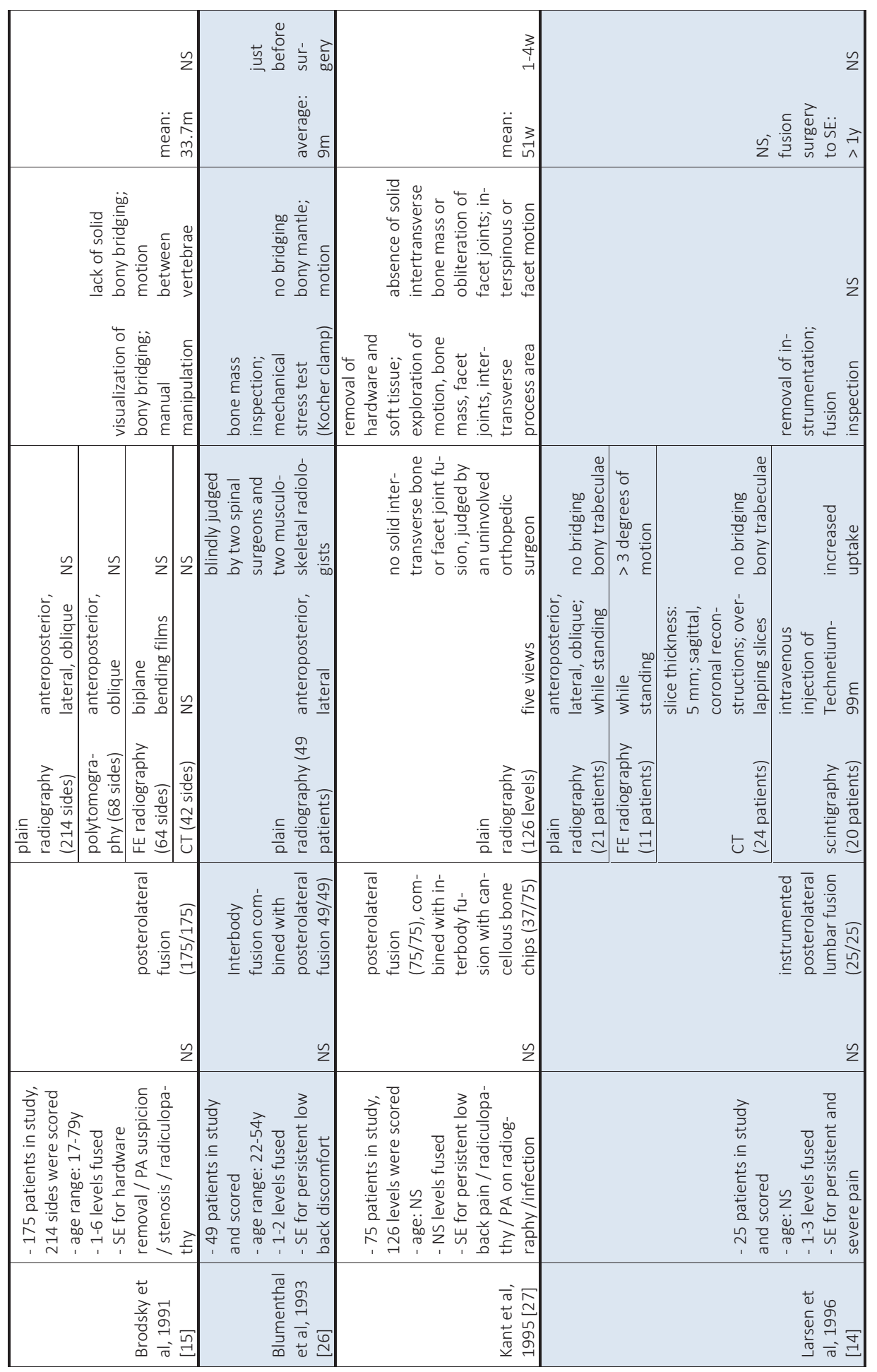




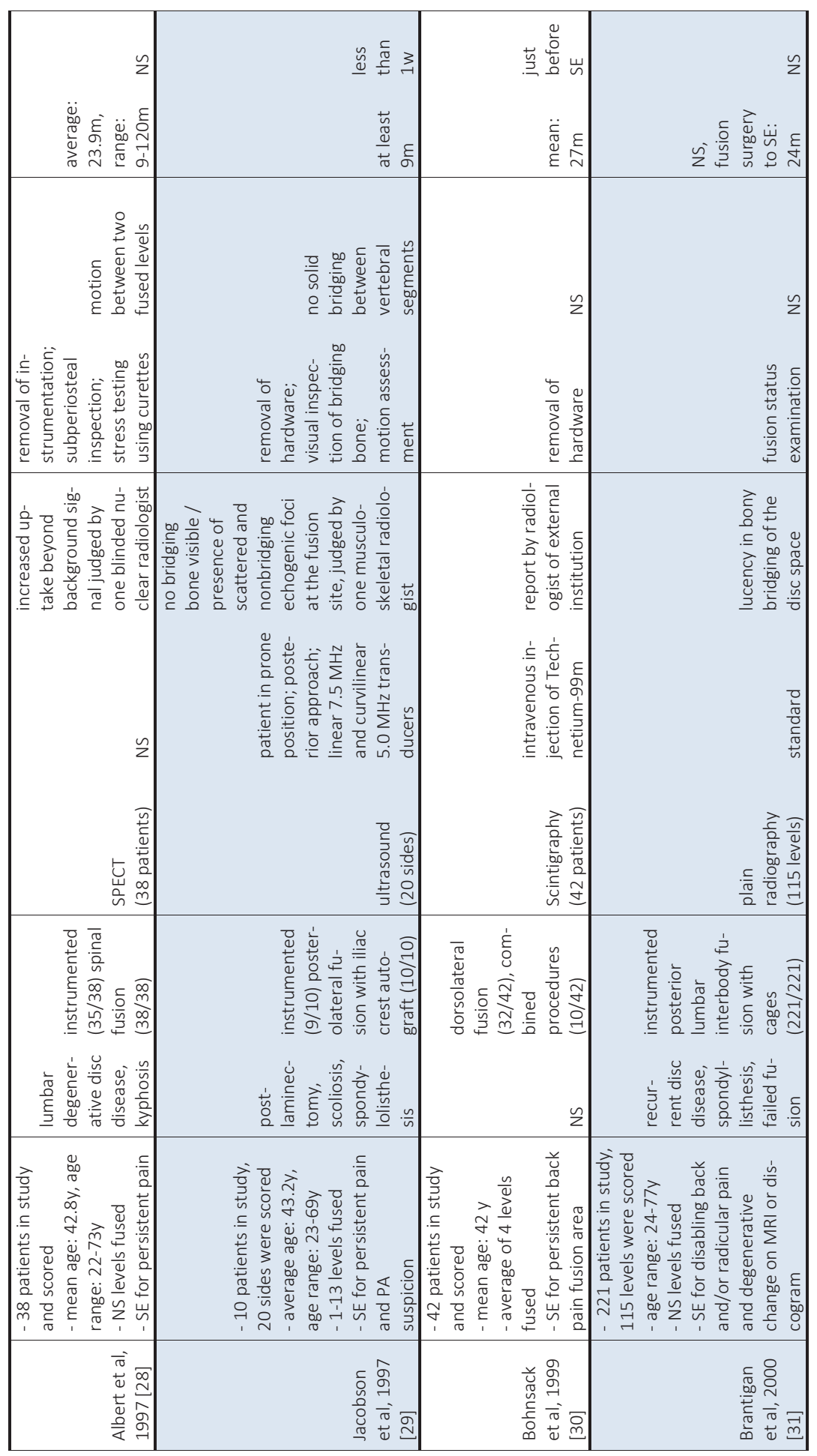




\begin{tabular}{|c|c|c|c|c|}
\hline 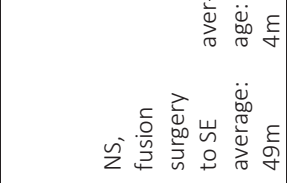 & \multicolumn{2}{|c|}{ 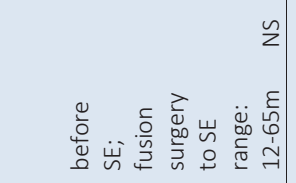 } & \multicolumn{2}{|r|}{ 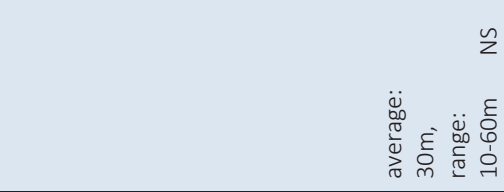 } \\
\hline 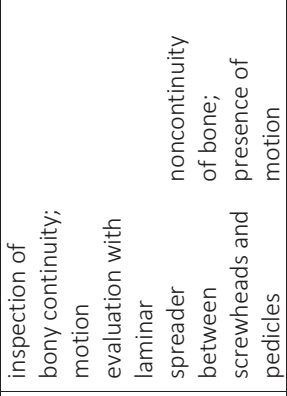 & \multicolumn{4}{|c|}{ 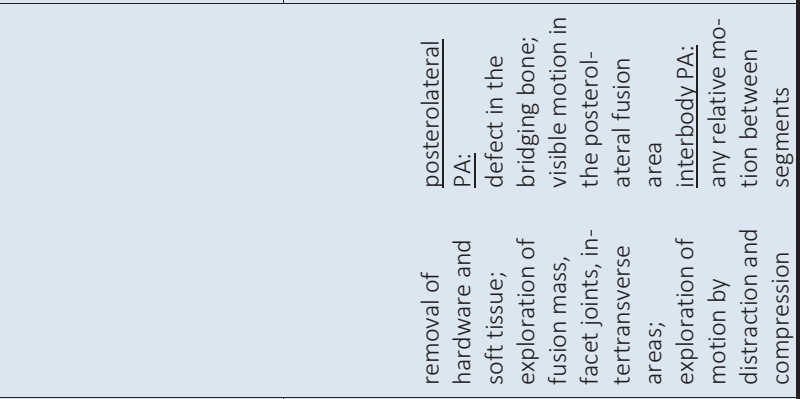 } \\
\hline \multirow[t]{2}{*}{ 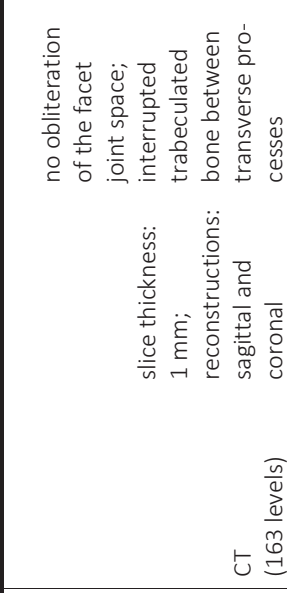 } & \multicolumn{2}{|c|}{ 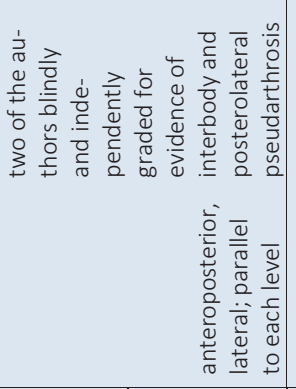 } & \multicolumn{2}{|c|}{ 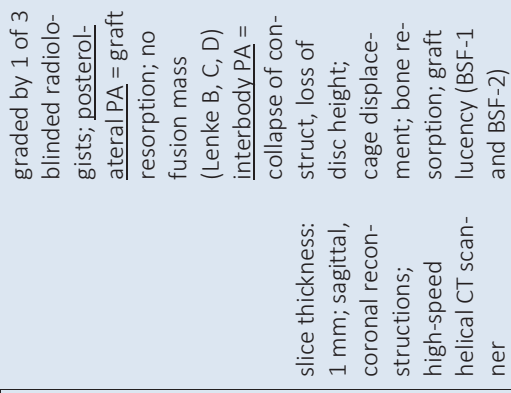 } \\
\hline & 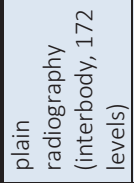 & 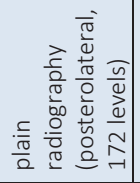 & 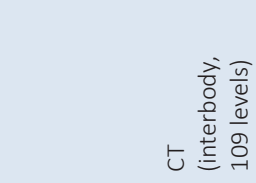 & 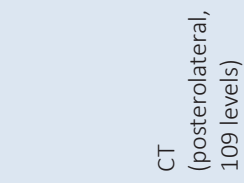 \\
\hline 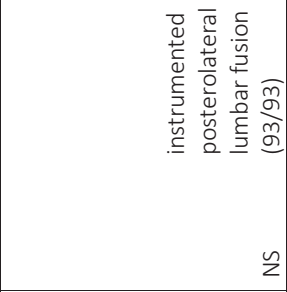 & \multicolumn{4}{|c|}{ 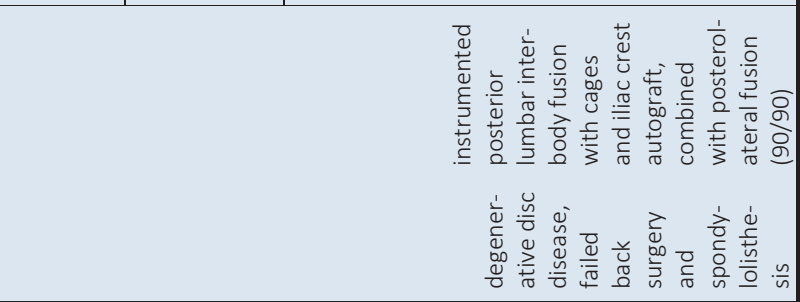 } \\
\hline 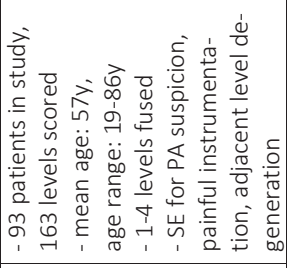 & \multicolumn{4}{|c|}{ 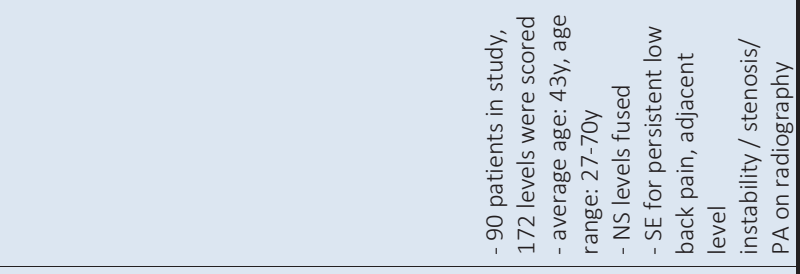 } \\
\hline 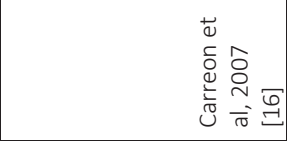 & & & & 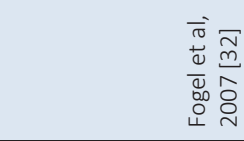 \\
\hline
\end{tabular}




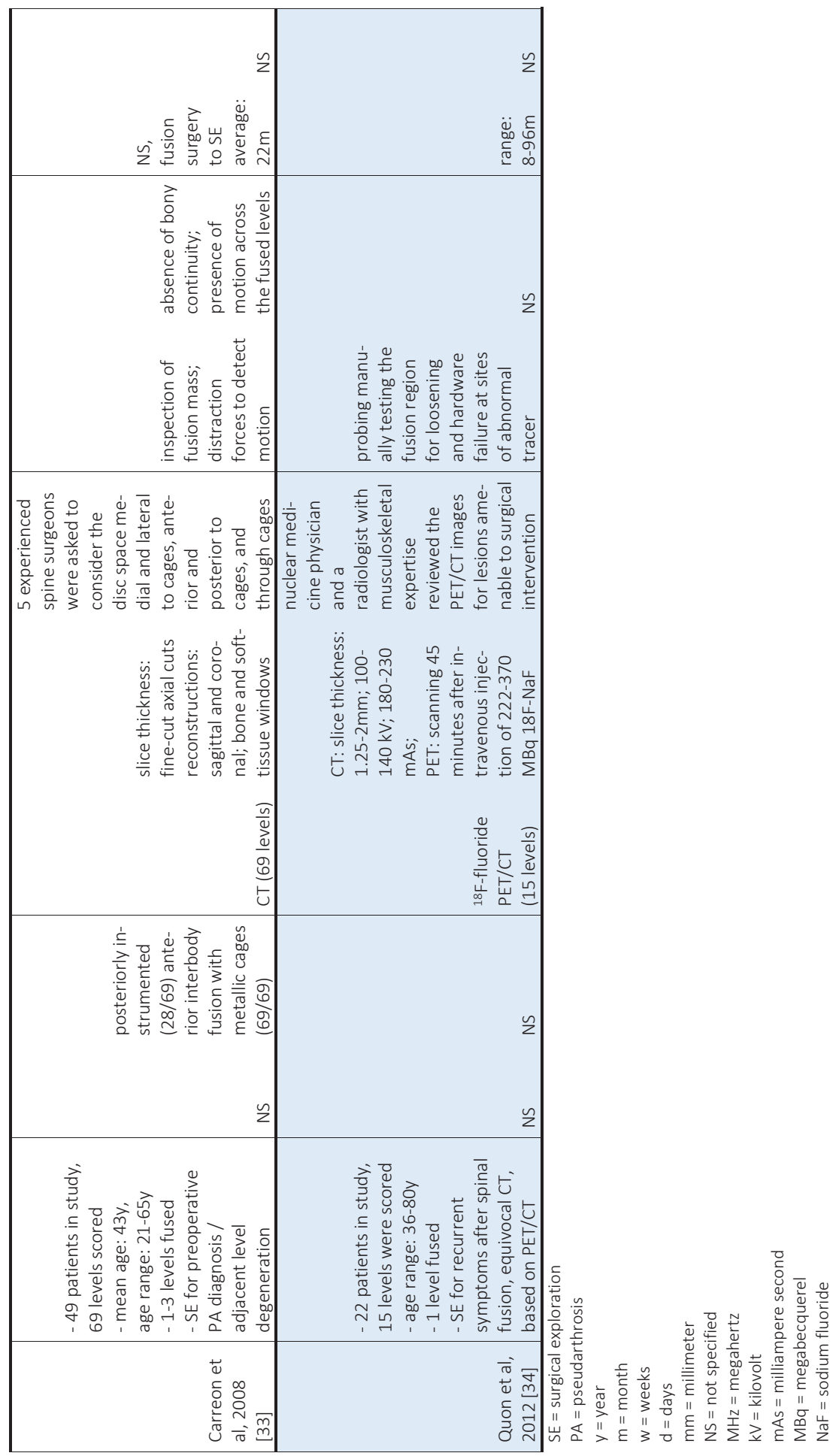




\section{Methodological quality assessment}

Table 2 displays the quality assessment according to QUADAS-2. An overview of the distribution of QUADAS-2 scores is presented in Figure 2. Risk of bias on 'flow and timing', 'patient selection', 'index test' and 'reference standard' was classified as high or unclear in $58 \%$ of cases. Common weaknesses related to poor documentation of patient selection and description of the reference standard. Two studies were considered to have low risk of bias in all four domains. Concerns of applicability on 'patient selection', 'index test' and 'reference standard' was classified as high or unclear in $42 \%$ of cases. Three studies were considered to suffer from low applicability concerns over all three domains. 


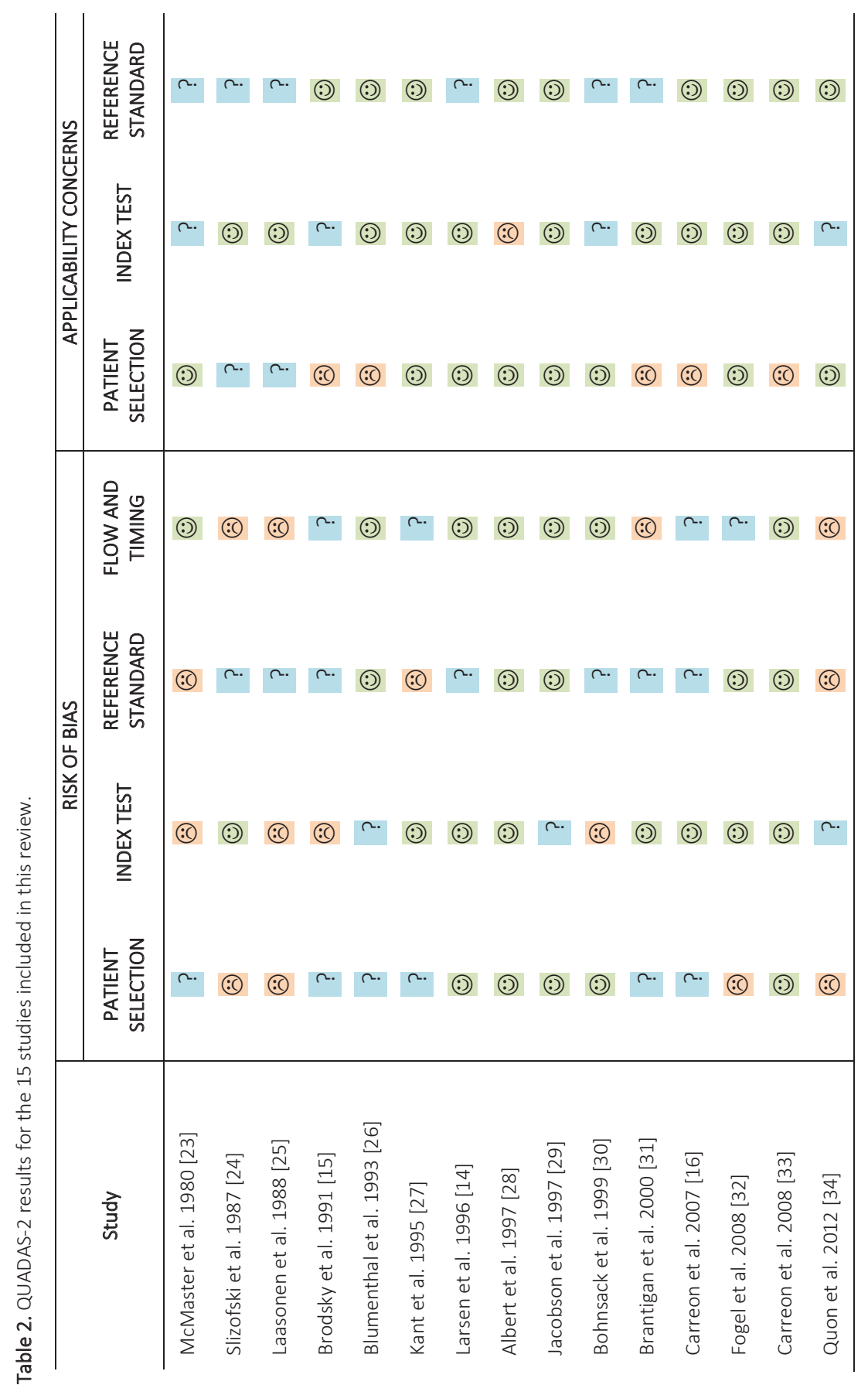




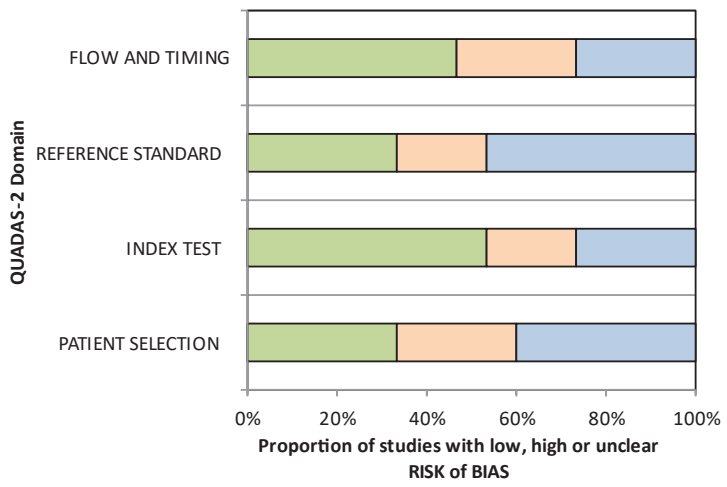

$\square$ Low $\square$ High $\square$ Unclear

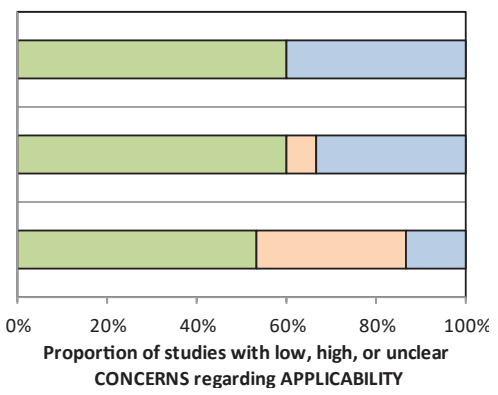

Figure 2. Stacked bar charts of QUADAS-2 scores presenting a quick overview of the methodological quality of the 15 included studies, expressed as a percentage of studies that met each criterion. For each quality domain, the proportion of included studies that suggest low, high, or unclear risk of bias and/or concerns regarding applicability are displayed in green, orange, and blue, respectively.

Data synthesis and statistical analysis

Table 3 shows the diagnostic accuracy values of the included studies, grouped per index test. 


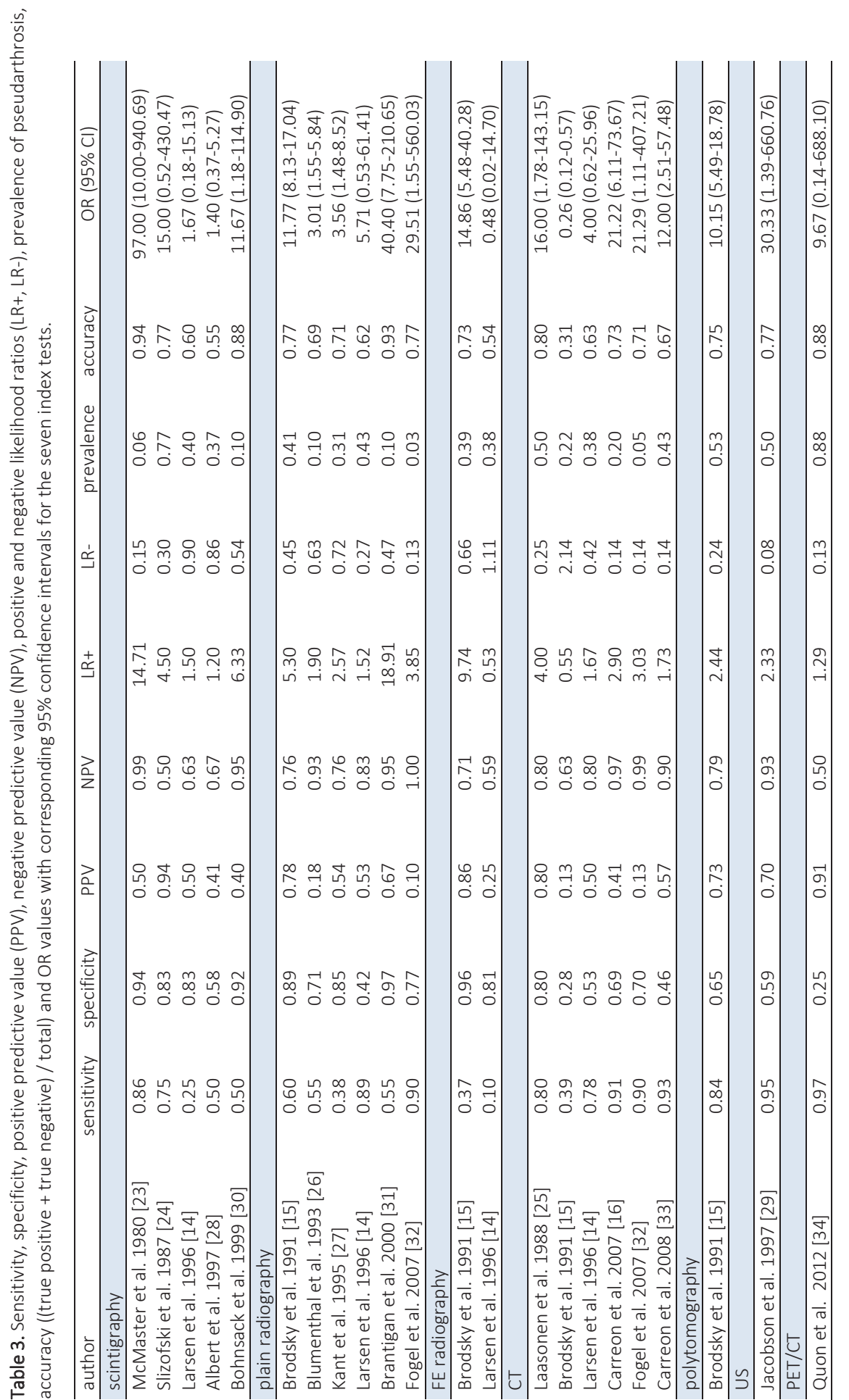


Inclusion in meta-analysis

The studies discussing the index tests SPECT $[24,28]$ and planar scintigraphy $[14,23,30]$ were considered for inclusion into subgroup meta-analysis further referred to as scintigraphy. McMaster et al. was not included because the time interval between fusion surgery and surgical exploration was deviating too much from the other studies. The remaining four studies were pooled.

Six studies were considered for inclusion in meta-analysis for plain radiography [14, 15, $26,27,31,32]$. Fogel et al. was excluded since the low prevalence of pseudarthrosis made the study population incomparable to the other studies (see Table 3). The remaining five studies were considered comparable enough to be pooled. Two articles diagnosed pseudarthrosis per patient $[14,26]$, two per level [27, 31] and one per side [15]. We chose to pool these studies despite differences in analysis region since we were mainly interested in the correlation between findings on imaging and surgical exploration. Using the same rationale, no distinction was made between studies on posterolateral and interbody fusion.

Two articles were considered for FE radiography meta-analysis [14, 15]. Apart from differences in analysis regions, the study characteristics were considered comparable and the studies were therefore pooled.

Six articles were considered for inclusion in CT meta-analysis [14-16, 25, 32, 33]. The study of Brodsky et al. was excluded for lack of sagittal and coronal reconstructions, essential in the assessment of interbody bony fusion [14, 16, 33, 35]. Laasonen et al. and Larsen et al. were excluded on slice thickness. Thicknesses of $5 \mathrm{~mm}$ and $6 \mathrm{~mm}$ were used respectively, while bony bridging should be assessed using thin slice CT to be reliable [16, $32,33,35]$. Fogel et al. was excluded for low prevalence of pseudarthrosis compared to the other studies. The posterolateral fusion patient group of Carreon et al. [16] and the interbody fusion patient group Carreon et al. [33] were pooled for CT.

Figure 3 shows a forest plot of the studies selected for subgroup meta-analysis, with their respective weights and resulting pooled OR's. Index tests for which only one study was identified, i.e. US, polytomography, ${ }^{18} \mathrm{~F}$-fluoride PET/CT [15, 29, 34], could inevitably not undergo subgroup meta-analysis. These single studies were however evaluated on the same grounds and if considered reliable, included in Table 4 to complement the metaanalysis results. This was only the case for the study on polytomography [15]. For the study on US [29], the authors considered that with the evaluation of 10 patients only, US was not investigated thoroughly enough for pseudarthrosis detection. In the ${ }^{18} \mathrm{~F}$-fluoride PET/CT study [34] the reference standard was either surgical exploration or clinical follow-up, based on the index test outcome. This introduced a bias in the patient population that underwent surgical exploration; only patients with a suspicion of pseudarthrosis on ${ }^{18} \mathrm{~F}$-fluoride PET/CT were surgically explored and used to calculate diagnostic accuracy. 


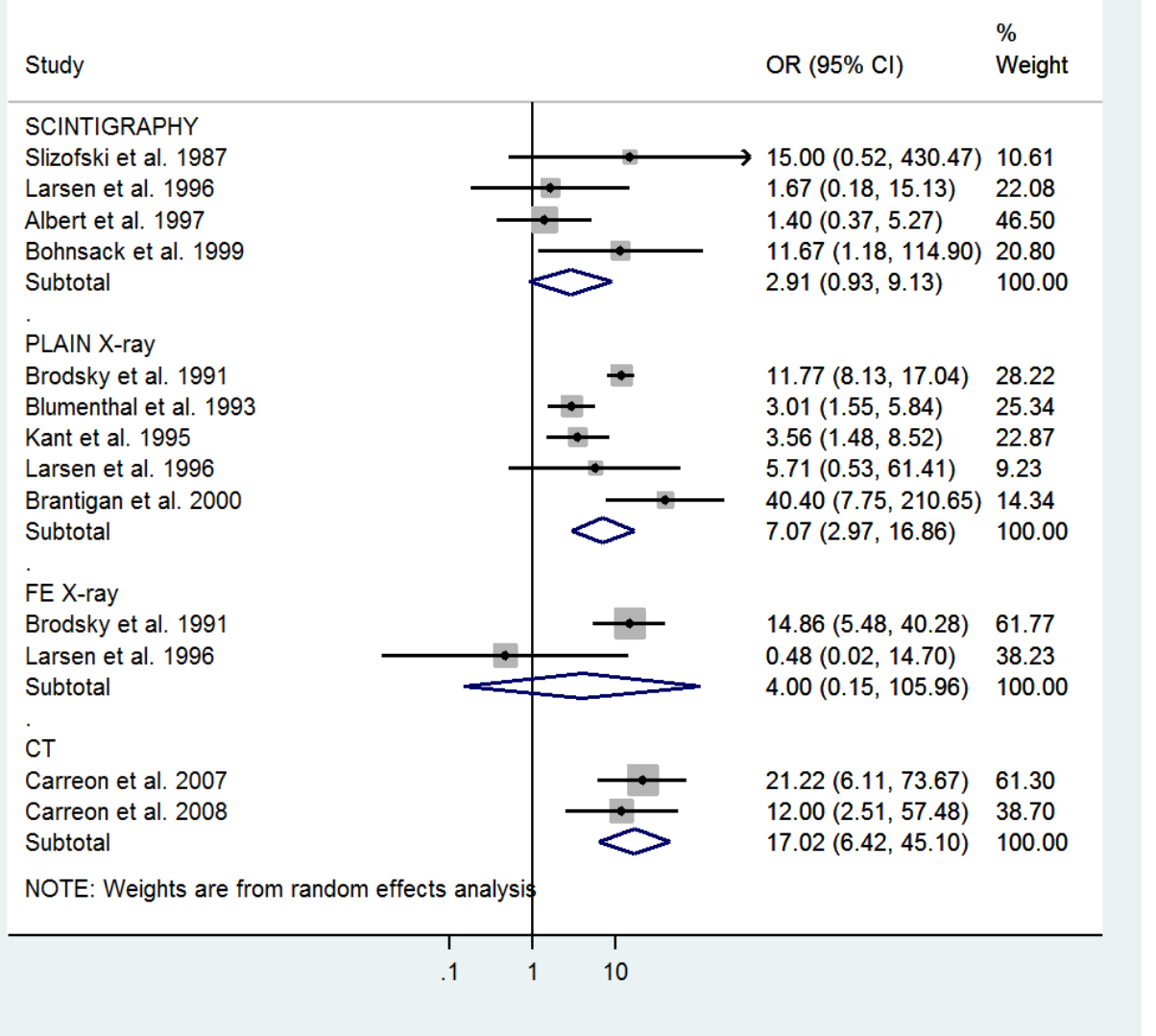

Figure 3. Forest plot of the included studies in the meta-analysis per modality. The size of each square is proportional to the study's weight.

Table 4. Overview of OR's as determined from included studies.

\begin{tabular}{lccc}
\hline & $\begin{array}{c}\text { Number } \\
\text { of studies }\end{array}$ & $\begin{array}{c}\text { Number } \\
\text { of patients }\end{array}$ & (Pooled) OR (95\% Cl) \\
\hline Scintigraphy $[14,24,28,30]$ & 4 & 93 & $2.91(0.93-9.13)$ \\
Plain radiography [14, 15, 26, 27, 31] & 5 & 398 & $7.07(2.97-16.86)$ \\
FE radiography [14, 15] & 2 & 75 & $4.00(0.15-105.96)$ \\
CT [16, 33] & 2 & 142 & $17.02(6.42-45.10)$ \\
Polytomography [15] & 1 & 68 & $10.15(5.49-18.78)$ \\
\hline
\end{tabular}




\section{DISCUSSION}

This systematic review summarizes studies in literature that investigated the diagnostic accuracy of imaging modalities to detect pseudarthrosis after thoracolumbar spinal fusion with surgical exploration as reference standard. Diagnostic accuracy values of individual studies were determined, and for studies of the same modality that were clinically comparable, a pooled OR was calculated.

Patients after spinal fusion can be monitored by several modalities. Plain radiographs attempt to reveal deficient morphology of the fusion mass as a sign of pseudarthrosis. However, plain radiographs are projections only $[35,36]$ whereas pseudarthrosis is a threedimensional problem. The pooled OR of radiography was 7.07. In FE radiography, radiographs are made during flexion and extension of the spinal column to detect motion in the operated segment as a sign of pseudarthrosis. Cases exist where no signs of pseudarthrosis were found on plain radiography, CT and MRI, but FE radiography detected the pseudarthrosis by unveiling motion between the segments [37]. However, on the other hand, absence of motion does not necessarily correspond with solid fusion and the presence of motion is not directly related to pseudarthrosis [12, 38-40]. Furthermore, no consensus exists on the threshold of allowable motion in a fused segment [40-42]. With a pooled OR of 4.00, FE radiography does not seem to outperform plain radiography. In polytomography, several radiographs along different sectional planes are taken. Going from a single slice in radiography to several planes in polytomography, the OR increased to 10.15. However, polytomography seems to be outdated by CT developments and currently not frequently used. CT offers three-dimensional osseous detail [33, 35]. After meta-analysis, CT was the modality with the highest OR in this review. Besides detection of bridging trabecular bone, CT is able to detect subsidence and lucency around fusion material as possible signs of pseudarthrosis [35]. On the downside, assessment can be complicated by artefacts when metallic cages and/or instrumentation are used [14, 32, 33, 35]. Technological improvements such as iterative reconstruction and dual-energy scanning are likely to improve accuracy [43]. Whether CT alone is sufficient for clinical decision making is under debate, Choudhri et al. stated that multiple modalities should be considered for the noninvasive evaluation of symptomatic patients with suspected failure of spinal fusion [38]. US can demonstrate callus formation and bone healing [44, 45]. Although the first study assessing the role of US for pseudarthrosis detection in 10 patients seemed promising in 1997 [29], it has been the only study since.

Pseudarthrosis diagnosis can also be based on abnormalities in bone metabolism. Studies on SPECT and planar scintigraphy were grouped together in meta-analysis since both modalities use technetium-99m ( ${ }^{99 \mathrm{~m}} \mathrm{Tc}$ )-labelled phosphonates as tracer. ${ }^{99 \mathrm{~m}} \mathrm{Tc}$-labelled phosphonates are adsorbed onto or into the crystalline structure of hydroxyapatite to mark bone remodeling. With a pooled OR of 2.91, scintigraphy amounted to the lowest OR value after subgroup meta-analyses. An analog to ${ }^{99 \mathrm{~m}} \mathrm{Tc}$-labelled phosphonates is ${ }^{18} \mathrm{~F}$ - 
fluoride. Both tracers have similar uptake mechanisms [46] but ${ }^{18} \mathrm{~F}$-fluoride decays via positron emission and can therefore be imaged by PET. Compared to ${ }^{99 \mathrm{~m} T C}$ SPECT, ${ }^{18} \mathrm{~F}$ fluoride PET provides higher resolution, higher sensitivity and better quantification capabilities [47]. PET combined with CT allows localization of abnormal uptake which might enhance discriminative power [6]. Quon et al. evaluated PET/CT as index test for pseudarthrosis diagnosis [34]. The results seem promising but studies of higher methodological quality should be conducted to draw firmer conclusions on its value in pseudarthrosis diagnosis.

In the database search, one paper evaluating MRI [48] and one paper evaluating RSA as index test [49] were identified but not included. In MRI, bridging bone between endplates can be visualized [50] and changes in the vertebral body marrow signal as a sign of functional instability can be detected $[48,51]$. On the downside, metal instrumentation complicates pseudarthrosis assessment in MRI. Length of follow-up was too short for the study of Lang et al. to be included. RSA is able to accurately quantify micromovements of vertebrae relative to each other, to evaluate lumbosacral stability $[38,42]$. The study of Pape et al. could not be used to calculate the diagnostic accuracy of RSA for pseudarthrosis detection since all patients attained fusion.

A strength of the present review was that the patient populations of the included studies resemble patient populations that would undergo these tests in clinical practice to either confirm or exclude pseudarthrosis, since all suffered from persisting or recurrent pain after spinal fusion. The methodological choice to only include studies that compared an index modality to the gold standard of surgical exploration was a strength on one hand since it is the most valid way to assess the diagnostic accuracy of a modality [14]. However, it was a weakness on the other hand, since it meant the exclusion of newer studies that evaluate state-of-the-art modalities. The study design of using surgical exploration as gold standard is no longer ethical or practical in clinical practice. As a result, the value of state-of-the-art modalities could not be discussed in this review and are still left to be evaluated. Another weakness of the study was that studies in meta-analysis, although relatively comparable, did show differences in spinal fusion technique, types of cages and instrumentation, imaging characteristics, pseudarthrosis definition, experience of the observers and patient characteristics. Especially the time interval between spinal fusion and index test was highly variable between studies. Furthermore, the interpretation of index test results was incomplete in some studies. Imaging findings were reported but not always classified as either pseudarthrosis or fused. In these cases, the cut-off point was determined by the writers of this review, which is arbitrary, although not necessarily far from clinical practice. Studies also reported poorly on patient population inclusion criteria. Lack of information may have led to incorrect inclusion of studies in meta-analyses and weakens the findings of this review. 
To conclude, with a pooled OR of 17.02 , CT can be considered the most accurate noninvasive imaging modality for the detection of pseudarthrosis after spinal fusion from this review. 


\section{REFERENCES}

1. Volinn E. The epidemiology of low back pain in the rest of the world. Spine. 1997; 22(15):1798.

2. Vos. Global, regional, and national incidence, prevalence, and years lived with disability for 301 acute and chronic diseases and injuries in 188 countries, 1990-2013: a systematic analysis for the Global Burden of Disease Study 2013. Lancet (London, England). 2015; 386(9995):743-800.

3. Grubb SA, Lipscomb HJ. Results of lumbosacral fusion for degenerative disc disease with and without instrumentation. Two- to five-year follow-up. Spine. 1992; 17(3):349-355.

4. O'Beirne J, O'Neill D, Gallagher J, Williams DH. Spinal fusion for back pain: a clinical and radiological review. Journal of spinal disorders. 1992; 5(1):32-38.

5. Larsen JM, Capen DA. Pseudarthrosis of the Lumbar Spine. The Journal of the American Academy of Orthopaedic Surgeons. 1997; 5(3):153-162.

6. Rager O, Schaller K, Payer M, Tchernin D, Ratib O, Tessitore E. SPECT/CT in differentiation of pseudarthrosis from other causes of back pain in lumbar spinal fusion: report on 10 consecutive cases. Clinical nuclear medicine. 2012; 37(4):339-343.

7. Raizman NM, O'Brien JR, Poehling-Monaghan KL, Yu WD. Pseudarthrosis of the spine. The Journal of the American Academy of Orthopaedic Surgeons. 2009; 17(8):494-503.

8. Heggeness MH, Esses SI. Classification of pseudarthroses of the lumbar spine. Spine. 1991; 16(8 Suppl):S449-454.

9. Rothman RH, Booth R. Failures of spinal fusion. The Orthopedic clinics of North America. 1975; 6(1):299304.

10. Brown CW, Orme TJ, Richardson HD. The rate of pseudarthrosis (surgical nonunion) in patients who are smokers and patients who are nonsmokers: a comparison study. Spine. 1986; 11(9):942-943.

11. Finnegan WJ, Fenlin JM, Marvel JP, Nardini RJ, Rothman RH. Results of surgical intervention in the symptomatic multiply-operated back patient. Analysis of sixty-seven cases followed for three to seven years. The Journal of bone and joint surgery American volume. 1979; 61(7):1077-1082.

12. Frymoyer JW, Hanley EN, Jr., Howe J, Kuhlmann D, Matteri RE. A comparison of radiographic findings in fusion and nonfusion patients ten or more years following lumbar disc surgery. Spine. 1979; 4(5):435-440.

13. Waddell G, Kummel EG, Lotto WN, Graham JD, Hall H, McCulloch JA. Failed lumbar disc surgery and repeat surgery following industrial injuries. The Journal of bone and joint surgery American volume. 1979; 61(2):201-207.

14. Larsen JM, Rimoldi RL, Capen DA, Nelson RW, Nagelberg S, Thomas JC, Jr. Assessment of pseudarthrosis in pedicle screw fusion: a prospective study comparing plain radiographs, flexion/extension radiographs, CT scanning, and bone scintigraphy with operative findings. Journal of spinal disorders. 1996; 9(2):117120.

15. Brodsky AE, Kovalsky ES, Khalil MA. Correlation of radiologic assessment of lumbar spine fusions with surgical exploration. Spine. 1991; 16(6 Suppl):S261-265.

16. Carreon LY, Djurasovic M, Glassman SD, Sailer P. Diagnostic accuracy and reliability of fine-cut CT scans with reconstructions to determine the status of an instrumented posterolateral fusion with surgical exploration as reference standard. Spine. 2007; 32(8):892-895. 
17. Hutton B, Salanti G, Caldwell DM, Chaimani A, Schmid CH, Cameron C, et al. The PRISMA extension statement for reporting of systematic reviews incorporating network meta-analyses of health care interventions: checklist and explanations. Annals of internal medicine. 2015; 162(11):777-784.

18. Liberati A, Altman DG, Tetzlaff J, Mulrow C, Gotzsche PC, loannidis JP, et al. The PRISMA statement for reporting systematic reviews and meta-analyses of studies that evaluate health care interventions: explanation and elaboration. Journal of clinical epidemiology. 2009; 62(10):e1-34.

19. Whiting PF, Rutjes AW, Westwood ME, Mallett S, Deeks JJ, Reitsma JB, et al. QUADAS-2: a revised tool for the quality assessment of diagnostic accuracy studies. Annals of internal medicine. 2011; 155(8):529-536.

20. Higgins JP, Green S. Cochrane handbook for systematic reviews of interventions: John Wiley \& Sons, 2011.

21. Ma X, Nie L, Cole SR, Chu H. Statistical methods for multivariate meta-analysis of diagnostic tests: An overview and tutorial. Statistical methods in medical research. 2016; 25(4):1596-1619.

22. Glas AS, Lijmer JG, Prins MH, Bonsel GJ, Bossuyt PM. The diagnostic odds ratio: a single indicator of test performance. Journal of clinical epidemiology. 2003; 56(11):1129-1135.

23. McMaster MJ, Merrick MV. The scintigraphic assessment of the scoliotic spine after fusion. The Journal of bone and joint surgery British volume. 1980; 62-B(1):65-72.

24. Slizofski WJ, Collier BD, Flatley TJ, Carrera GF, Hellman RS, Isitman AT. Painful pseudarthrosis following lumbar spinal fusion: detection by combined SPECT and planar bone scintigraphy. Skeletal radiology. 1987; 16(2):136-141.

25. Laasonen EM, Soini J. Low-back pain after lumbar fusion. Surgical and computed tomographic analysis. Spine. 1989; 14(2):210-213.

26. Blumenthal SL, Gill K. Can lumbar spine radiographs accurately determine fusion in postoperative patients? Correlation of routine radiographs with a second surgical look at lumbar fusions. Spine. 1993; 18(9):1186-1189.

27. Kant AP, Daum WJ, Dean SM, Uchida T. Evaluation of lumbar spine fusion. Plain radiographs versus direct surgical exploration and observation. Spine. 1995; 20(21):2313-2317.

28. Albert TJ, Pinto M, Smith MD, Balderston RA, Cotler JM, Park CH. Accuracy of SPECT scanning in diagnosing pseudoarthrosis: a prospective study. Journal of spinal disorders. 1998; 11(3):197-199.

29. Jacobson JA, Starok M, Pathria MN, Garfin SR. Pseudarthrosis: US evaluation after posterolateral spinal fusion: work in progress. Radiology. 1997; 204(3):853-858.

30. Bohnsack M, Gosse F, Ruhmann O, Wenger K. The value of scintigraphy in the diagnosis of pseudarthrosis after spinal fusion surgery. Journal of spinal disorders. 1999; 12(6):482-484.

31. Brantigan JW, Steffee AD, Lewis ML, Quinn LM, Persenaire JM. Lumbar interbody fusion using the Brantigan I/F cage for posterior lumbar interbody fusion and the variable pedicle screw placement system: twoyear results from a Food and Drug Administration investigational device exemption clinical trial. Spine. 2000; 25(11):1437-1446.

32. Fogel GR, Toohey JS, Neidre A, Brantigan JW. Fusion assessment of posterior lumbar interbody fusion using radiolucent cages: X-ray films and helical computed tomography scans compared with surgical exploration of fusion. The spine journal : official journal of the North American Spine Society. 2008; 8(4):570577.

33. Carreon LY, Glassman SD, Schwender JD, Subach BR, Gornet MF, Ohno S. Reliability and accuracy of finecut computed tomography scans to determine the status of anterior interbody fusions with metallic cages. The spine journal: official journal of the North American Spine Society. 2008; 8(6):998-1002. 
34. Quon A, Dodd R, lagaru A, de Abreu MR, Hennemann S, Alves Neto JM, et al. Initial investigation of (1)(8)F$\mathrm{NaF}$ PET/CT for identification of vertebral sites amenable to surgical revision after spinal fusion surgery. European journal of nuclear medicine and molecular imaging. 2012; 39(11):1737-1744.

35. Williams AL, Gornet MF, Burkus JK. CT evaluation of lumbar interbody fusion: current concepts. AJNR American journal of neuroradiology. 2005; 26(8):2057-2066.

36. Schuler TC, Subach BR, Branch CL, Foley KT, Burkus JK. Segmental lumbar lordosis: manual versus computer-assisted measurement using seven different techniques. Journal of spinal disorders \& techniques. 2004; 17(5):372-379.

37. Shen FH, Samartzis D. Assessment of lumbar fusion: importance of dynamic plain standing x-rays. Journal of the American College of Surgeons. 2008; 207(6):955-956.

38. Choudhri TF, Mummaneni PV, Dhall SS, Eck JC, Groff MW, Ghogawala Z, et al. Guideline update for the performance of fusion procedures for degenerative disease of the lumbar spine. Part 4: radiographic assessment of fusion status. Journal of neurosurgery Spine. 2014; 21(1):23-30.

39. McAfee PC, Boden SD, Brantigan JW, Fraser RD, Kuslich SD, Oxland TR, et al. Symposium: a critical discrepancy-a criteria of successful arthrodesis following interbody spinal fusions. Spine. 2001; 26(3):320-334.

40. Santos ER, Goss DG, Morcom RK, Fraser RD. Radiologic assessment of interbody fusion using carbon fiber cages. Spine. 2003; 28(10):997-1001.

41. Hipp JA, Reitman CA, Wharton N. Defining pseudoarthrosis in the cervical spine with differing motion thresholds. Spine. 2005; 30(2):209-210.

42. Selby MD, Clark SR, Hall DJ, Freeman BJ. Radiologic assessment of spinal fusion. The Journal of the American Academy of Orthopaedic Surgeons. 2012; 20(11):694-703.

43. Stradiotti P, Curti A, Castellazzi G, Zerbi A. Metal-related artifacts in instrumented spine. Techniques for reducing artifacts in $\mathrm{CT}$ and $\mathrm{MRI}$ : state of the art. European spine journal : official publication of the European Spine Society, the European Spinal Deformity Society, and the European Section of the Cervical Spine Research Society. 2009; 18 Suppl 1:102-108.

44. Eyres KS, Bell MJ, Kanis JA. Methods of assessing new bone formation during limb lengthening. Ultrasonography, dual energy X-ray absorptiometry and radiography compared. The Journal of bone and joint surgery British volume. 1993; 75(3):358-364.

45. Maffulli N, Thornton A. Ultrasonographic appearance of external callus in long-bone fractures. Injury. 1995; 26(1):5-12.

46. Wong KK, Piert M. Dynamic bone imaging with 99mTc-labeled diphosphonates and 18F-NaF: mechanisms and applications. Journal of nuclear medicine : official publication, Society of Nuclear Medicine. 2013; 54(4):590-599.

47. Rahmim A, Zaidi H. PET versus SPECT: strengths, limitations and challenges. Nuclear medicine communications. 2008; 29(3):193-207.

48. Lang P, Chafetz N, Genant HK, Morris JM. Lumbar spinal fusion. Assessment of functional stability with magnetic resonance imaging. Spine. 1990; 15(6):581-588.

49. Pape D, Adam F, Fritsch E, Muller K, Kohn D. Primary lumbosacral stability after open posterior and endoscopic anterior fusion with interbody implants: a roentgen stereophotogrammetric analysis. Spine. 2000; 25(19):2514-2518.

50. Kroner AH, Eyb R, Lange A, Lomoschitz K, Mahdi T, Engel A. Magnetic resonance imaging evaluation of posterior lumbar interbody fusion. Spine. 2006; 31(12):1365-1371. 
51. Steinmann JC, Herkowitz HN. Pseudarthrosis of the spine. Clinical orthopaedics and related research. 1992(284):80-90. 


\section{CHAPTER 3}

\section{Pseudarthrosis after lumbar spinal fusion: the role of ${ }^{18} \mathrm{~F}$-fluoride PET/CT}

Eur J Nucl Med Mol Imaging. 2015 Nov;42(12):1891-8.

doi: 10.1007/s00259-015-3154-y

Marloes JM Peters, Paul C Willems, René E Weijers, Roel Wierts, Elisabeth MC Jutten, Christian Urbach, Chris JC Arts, Lodewijk W van Rhijn, Boudewijn T Brans 


\section{ABSTRACT}

\section{PURPOSE}

Painful pseudarthrosis is one of the most important indications for (revision-) surgery after spinal fusion procedures. It is of great clinical importance to ascertain if pseudarthrosis is the source of the recurrent pain because this may warrant revision surgery. The correlation between findings on conventional imaging (plain radiography and CT) and clinical well-being has been shown to be moderate. The goal of this study was to investigate the possible role of ${ }^{18} \mathrm{~F}$-fluoride PET in patients after lumbar spinal interbody fusion by relating $\mathrm{PET} / \mathrm{CT}$ findings to clinical function and pain.

\section{METHODS}

A cohort of 36 patients was retrospectively included in the study after ${ }^{18} \mathrm{~F}$-fluoride PET/CT, either for persistent or recurrent low back pain $(n=18)$ or during routine postoperative investigation ( $n=18$ ), between 9 to 76 months and 11 to 14 months after posterior lumbar interbody fusion respectively. Sixty minutes after intravenous injection of 156-263 MBq (mean $199 \mathrm{MBq}$; median $196 \mathrm{MBq})^{18} \mathrm{~F}$-fluoride, PET and CT-images were made with an integrated PET/CT scanner, followed by a diagnostic CT scan. Two observers independently scored the images. The number of bony bridges between vertebrae was scored on CT to quantify interbody fusion (0, 1 or 2$)$. Results of vertebral endplate and intervertebral disc space uptake were obtained, visually as well as semiquantitatively by means of ${ }^{18}$ F-fluoride PET. Findings on PET and CT were correlated to clinical well-being as measured by validated questionnaires concerning general daily functioning (Oswestry Disability Index), pain (Visual Analogue Scale) and general health status (EuroQol). Patients were divided into three categories based on these questionnaire scores.

\section{RESULTS}

No correlation could be observed between the degree of symptoms and fusion status. However, ${ }^{18}$ F-fluoride activity at the vertebral endplates was significantly higher in the lowest Oswestry Disability Index category (i.e., with the worst clinical performance), as compared to the other categories ( $p=0.01$ between categories 1 and 2 and 1 and 3 ). Results for Visual Analogue Scale and EuroQol were similar although less pronounced, with only SUV $\max$ Scores between category 1 and 2 being significantly different $(p=0.04)$.

\section{CONCLUSION}

We hypothesize that ${ }^{18} \mathrm{~F}$-fluoride PET/CT can provide support for the diagnosis of painful pseudarthrosis and could serve as a discriminary tool between symptomatic and asymptomatic pseudarthrosis for revision surgery, as CT defines consolidation status and PET pinpoints the 'stress reaction' at the vertebral endplates which significantly correlates with the Oswestry Disability Index. 


\section{INTRODUCTION}

Low back pain is a major global health and economic problem [1], with a 1-year prevalence ranging from 22 to $65 \%$ and life-time prevalence of up to $84 \%$ [2]. The direct costs in the Netherlands (15 million inhabitants), including patient care, medical procedures and medication, have been estimated to be 474 million euros in 2007 [3]. However, the yearly indirect costs caused by absence from work and early retirement in the Netherlands are manifold, i.e. 3.1 billion euros in 2007 [3].

Low back pain is mainly caused by degenerative spinal disorders, such as spondylolisthesis, degenerative scoliosis, degenerative disc disease, or recurrent disc herniations [4, 5]. If conservative measures, such as intensive exercise therapy, pain medication or brace immobilization fail, operative intervention is considered. Spinal fusion is a surgical procedure that aims to eliminate painful intervertebral motion by rigid fixation using metal implants and bone grafts, to create a definitive bony fusion. One of the most used spinal fusion techniques is posterior lumbar interbody fusion (PLIF) which is characterized by a posterior surgical approach. Although multiple improvements in the surgical technique have led to higher fusion rates, failed back surgery syndrome remains a substantial problem [6]. Pseudarthrosis is defined as the absence of solid bony fusion one year after the operation and occurs in at least $15 \%$ of primary lumbar fusions $[7,8]$. Pseudarthrosis is typically associated with low back pain or radicular pain as a result of continued motion $[6,7]$. However, pseudarthrosis may also be asymptomatic or symptoms may be atypical in a significant proportion of patients. Persistent or recurrent pain is one of the most important determinants in decision making for spinal revision surgery [9]. Therefore, it is essential to objectively relate pain symptoms to the degree of pseudarthrosis in order to justify revision surgery of the vertebral segments [10].

Surgical exploration is currently the gold standard for the detection of pseudarthrosis, but is highly invasive and recommended only in case of high suspicion of pseudarthrosis or hardware failure $[6,7,11-13]$. Computed tomography (CT) is a powerful imaging modality that allows the detection of well-established bony bridges between vertebrae. However, CT is of limited value for the diagnosis of an evolving, early staged pseudarthrosis, in patients with symptoms postoperatively. Carreon et al. compared CT data with revision surgical findings in 163 cases of posterolateral fusion and found that the diagnosis of bilateral fusion as determined using thin-sliced CT was confirmed upon exploration in $96 \%$ of all cases, indicating a high negative predictive value (NPV) for pseudarthrosis. However, the absence of fusion on one or both sides on CT was a poor predictor of pseudarthrosis upon surgical exploration (low positive predictive value (PPV)) [12]. Moreover, the association between CT findings and clinical symptoms is moderate [7, 14]. Nuclear bone scanning may provide earlier functional diagnosis, and can be performed by ${ }^{99 \mathrm{~m} T \mathrm{Tc}}$ labeled diphosphonates, suitable for SPECT/CT, or by ${ }^{18} \mathrm{~F}$-fluoride for positron emission tomography (PET). Both tracers have similar uptake mechanisms in newly formed bone 
or osteoid and are therefore indicators of osteoblastic activity. However, ${ }^{18} \mathrm{~F}$-fluoride $\mathrm{PET} / \mathrm{CT}$ may provide images with higher resolution, sensitivity and better quantitation capabilities as compared to ${ }^{99 \mathrm{~m} T C}$ SPECT/CT, necessary for evaluation of stress reactions and bone remodeling processes in the spine.

The hypothesis of this study was that ${ }^{18} \mathrm{~F}$-fluoride PET could play a role in objectively relating clinical symptoms to pseudarthrosis, as radiopharmaceutical activity functionally correlates best with disease activity. Therefore, ${ }^{18} \mathrm{~F}$-fluoride PET may help to discriminate between symptomatic and asymptomatic pseudarthrosis and serve as an indicator for revision surgery. To this end, we investigated the relation between ${ }^{18} \mathrm{~F}$-fluoride PET/CT and clinical symptoms after PLIF, not only in patients with persistent or recurrent back pain, but also in patients with minor or no pain.

\section{MATERIALS AND METHODS}

\section{Patients}

A total of 36 patients who had undergone PLIF were retrospectively included for the present analysis between June 2008 and October 2014. Eighteen of the patients suffered persistent or recurrent low back pain without an apparent cause on conventional scans and diagnostics ('persistent pain' group), at a variable time point after operation (range 9 to 76 months; mean 24.9 months; median 23 months). The other eighteen patients were recruited as a consecutive cohort during routine clinical and radiological ( $\mathrm{x}$-ray and $\mathrm{CT}$ ) investigation one year after PLIF ('postoperative' group; range 11 to 14 months; mean 12.4 months; median 12 months). Three patients had undergone PLIF surgery at two levels. Therefore, the total number of operated levels to be analyzed was 39. Operated levels were L3-L4 ( $=3)$, L4-L5 ( $=15)$ and L5-S1 $(n=21)$. In part, this was an extension of an already published previous pilot study [15].

\section{Posterior Lumbar Interbody Fusion (PLIF), surgical technique}

Under general anesthesia and in a prone position, the vertebral arches of the intended levels were identified under fluoroscopic control and exposed, using an open posterior lumbar approach. Nerve roots were decompressed by laminectomy and the intervertebral disc was excised. After thorough abrasion of the endplates, two 10-12 mm intervertebral cages (Capstone ${ }^{\circledR}$ PEEK, Medtronic, Memphis, U.S.A.), were filled with autologous bone from the vertebral lamina and inserted into the disc space. The remaining disc space was packed with additional autologous bone chips from the removed lamina. Next, the upper and lower vertebrae were fixed using four transpedicular screws connected to titanium rods (CD Legacy', Medtronic, Memphis, U.S.A.) for primary stabilization. 


\section{${ }^{18}$ F-fluoride PET/CT scan acquisition}

Sixty minutes after intravenous injection of 156-263 MBq (mean 199 MBq; median 196 $\mathrm{MBq}){ }^{18} \mathrm{~F}$-fluoride, PET and CT-images were acquired with an integrated PET/CT scanner (Gemini TF PET-CT, Philips, the Netherlands). After a low-dose attenuation-correction CT acquisition (120 kV, $30 \mathrm{mAs}$, slice thickness $4 \mathrm{~mm}$ ), a PET scan was made in three-dimensional mode, by acquiring two bed positions of five minutes, covering the lumbosacral spine. This was immediately followed by a diagnostic, non-contrast enhanced CT scan (64-slice helical, $120 \mathrm{kV}, 250 \mathrm{mAs}$, slice $1 \mathrm{~mm}$ thickness with increment of $0.8 \mathrm{~mm}$ ) of the fusion region. Standard filtered back projection CT reconstruction was performed. PET image reconstruction included both non-attenuated and CT-based attenuated data, using time-of-flight technology. Images were viewed and post processed on clinical software (EBW, Philips, the Netherlands), and further analyzed using dedicated research software (PMOD 3.0, PMOD Technologies Ltd, Zürich).

\section{${ }^{18}$ F-fluoride PET/CT data analysis}

The ${ }^{18} \mathrm{~F}$-fluoride PET/CT scans were evaluated by two independent, blinded observers (MP, BB) in order to determine a volume of interest (VOI) for ${ }^{18} \mathrm{~F}$-fluoride uptake calculation and to score bony bridging based on standard diagnostic CT scans. Afterwards, discrepancies between observers were resolved by consensus. Scoring was verified by an orthopedic surgeon (PW) and a musculoskeletal radiologist (RW). Interbody fusion between the upper and lower vertebrae was defined as the presence of a bone bridge (Figure 1) on both sides either within or around the cages (score 2), on one side within or around the cage, right or left (score 1), or no bridging (score 0 ) on the diagnostic CT scan (Figure 2). In each low-dose CT scan, three ellipsoid shaped VOIs were manually drawn following the contours of the vertebrae (slice thickness $4 \mathrm{~mm}$, short axis range: 40-50 $\mathrm{mm}$, long axis range: 55-65 $\mathrm{mm}$ ), including the intervertebral disc space and upper and lower endplates of the operated segment (Figure $3 \mathrm{~A}$ ). These VOIs were then transferred to the co-registered attenuation corrected PET-image (Figure 3B) and in each of these VOIs the SUV $\max$ was determined, i.e. activity at the upper, lower endplates and the intervertebral disc space $\left(S U V_{\max } U, S U V_{\max } L\right.$ and $S U V_{\max } D$ respectively), as well as activity ratios of upper and lower endplate to the intervertebral disc space (SUVratio $U$ and SUV ratio $L$ respectively). 


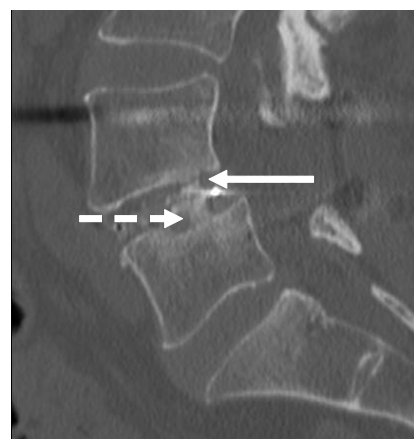

Figure 1. Intervertebral bony fusion scoring on CT. Example of a bony bridge between cage and lower vertebra (closed arrow), but not between the cage and the upper vertebra (dotted arrow).
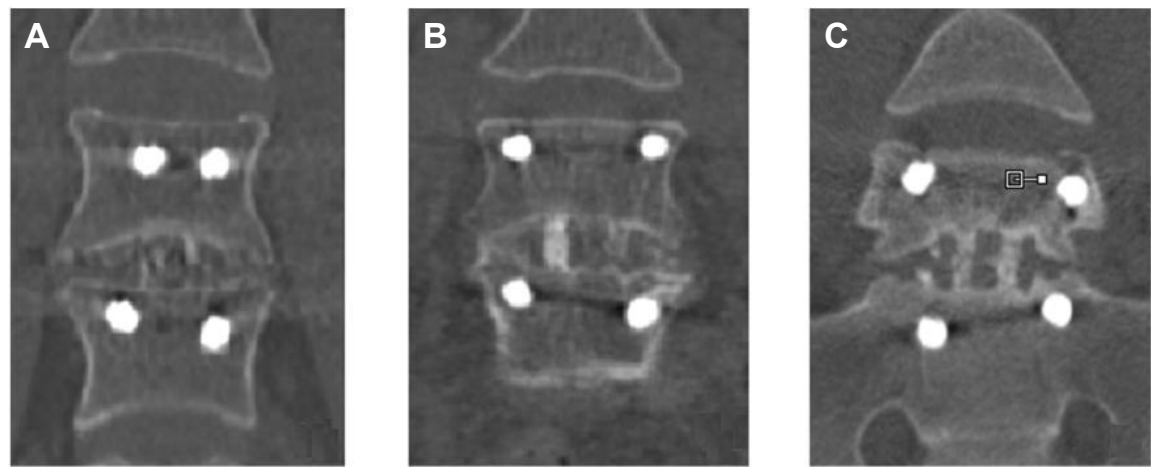

Figure 2. Examples of CT fusion score 0 (A), score 1 (B) and score 2 (C).
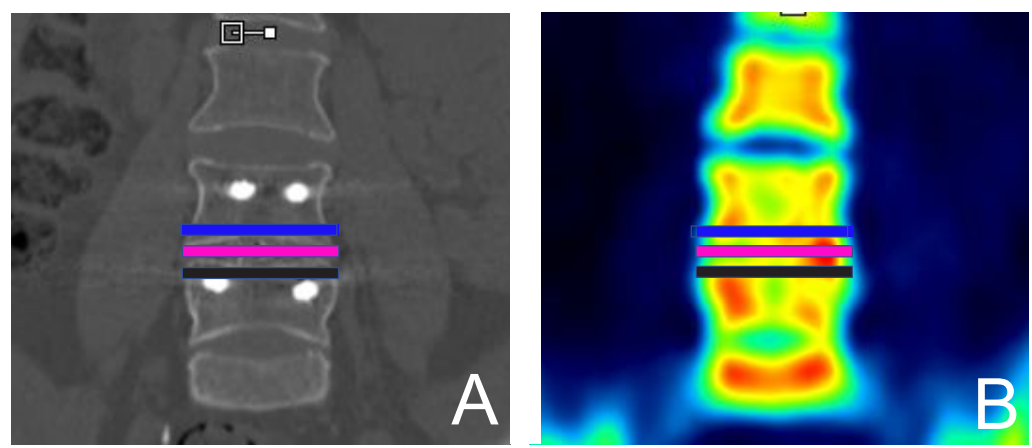

Figure 3. ${ }^{18} \mathrm{~F}$-fluoride PET analysis of the operated segment. Coronal CT (A) and PET (B) images of the lumbar spine after PLIF, in which three VOIs were drawn in the operated segment: the lower endplate of the cranial vertebra (black), the intervertebral disc space (pink) and the upper endplate of the caudal vertebra (blue). 


\section{Patient reported outcome measures}

Clinical well-being of the patients was evaluated using a set of validated questionnaires: 1. The Oswestry Disability Index (ODI) is the most commonly used outcome measure for low back pain and disability [16] and assess ten general daily tasks based on back related function with 6 possible answer each [17].

2. Pain in back and/or legs is reliably quantified by a Visual Analogue Scale (VAS) [9]. Patients were asked to express the amount of pain in their back, right leg and left leg separately on three scales ranging from 0 (no pain) to 100 (worst pain).

3. The EuroQol (EQ)-5D is the most commonly used instrument for measuring healthrelated quality of life in the Netherlands [18]. The questionnaire consists of five questions with three possible answers, each representing a domain (i.e. mobility, self-care, usual activities, pain/discomfort and anxiety/depression). The EQ-5D index score was calculated based on a Dutch value set, representative of the Dutch population with regard to age and gender.

To facilitate further analysis of the data, each questionnaire score was linearly re-scaled on a scale of 0 to 100, with 0 the worst possible score and 100 the best possible score. Patients were divided into categories based on their questionnaire score; patients with scores 0-40 were placed in category 1 (worst score), scores 40-60 in category 2 (intermediate score), and scores 60-100 in category 3 (best score) respectively.

\section{Statistical analysis}

Statistical evaluation was performed using IBM SPSS Statistics for Windows, Version 20.0 (Armonk, NY: IBM Corporation). To test the data for normality of distribution, the ShapiroWilk test was used. In case of normality, an independent t-test was used to test whether two samples originated from the same distribution. In case any of the involved parameters was not normally distributed, the Mann-Whitney U-test was used. P-values smaller than or equal to 0.05 were used to indicate a statistically significant difference.

\section{RESULTS}

Table 1 presents a summary of the key findings with the distribution of SUV, CT and questionnaire scores. As can be seen, a full range of symptomatic as well as asymptomatic patients was included. As expected, patients in follow-up who presented with persistent back pain showed worse questionnaire scores than patients routinely scanned postoperatively. For example, regarding back related function (ODI), $67 \%$ of patients in the persistent pain group fell in the intermediate or worst symptom category 1 or 2 , while this was only $11 \%$ in the postoperative group. Also important, there was high similarity between 
the questionnaire scores; a low or high ODI score consistently corresponded to a low/high VAS and a low/high EQ-5D score, with R-values of 0.9, 0.7 and 0.6 between ODI and VAS, ODI and EQ-5D and VAS and EQ-5D respectively.

Table 1. Key findings.

Persistent pain group

( $n=18)$
Postoperative group

$(n=18)$

\section{Time interval ${ }^{a}$}

primary PLIF surgery - PET/CT

$24.9(9-76)$

$12.4(11-14)$

\section{Imaging findings}

PET ${ }^{b}$

SUV $\max U$

SUV $V_{\max } \mathrm{D}$

SUV max $_{\text {L }}$

SUV ratio U

SUV ratio L

$14.3(4.4)$

$12.5(4.5)$

$14.3(5.3)$

$1.2(0.4)$

$1.2(0.5)$

14.9 (3.9)

$15.0(6.5)$

$14.0(3.6)$

$1.0(0.3)$

$1.0(0.3)$

$C T^{c}$

fusion score 0

fusion score 1

fusion score 2

7

3

12
$49.9(22.6)$
$50.7(23.6)$
$62.0(23.8)$

$82.6(12.5)$

$81.2(16.2)$

$83.4(14.0)$

a: [months], values as mean (range)

b: [-], values as mean (standard deviation)

c: [number of levels] 
Figure 4 shows the relation between the intervertebral fusion score on CT and the ODI scores. ODI scores were $53.1 \pm 25.3$ (mean \pm standard deviation) for fusion score $0 ; 78.6$ \pm 18.0 for score 1 and $66.0 \pm 25.4$ for score 2 . Only the difference in ODI score between fusion score 0 and 1 was statistically significant ( $p=0.017 ; p=0.226$ between scores 0 and 2 and $p=0.163$ between scores 1 and 2 ). Therefore, no consistent correlation could be observed between degree of pain symptoms and CT fusion score. For VAS and EQ-5D similar statistically significant results were obtained, with only statistically significant differences between groups 0 and 1 ( $p=0.03$ for both VAS and EQ-5D).

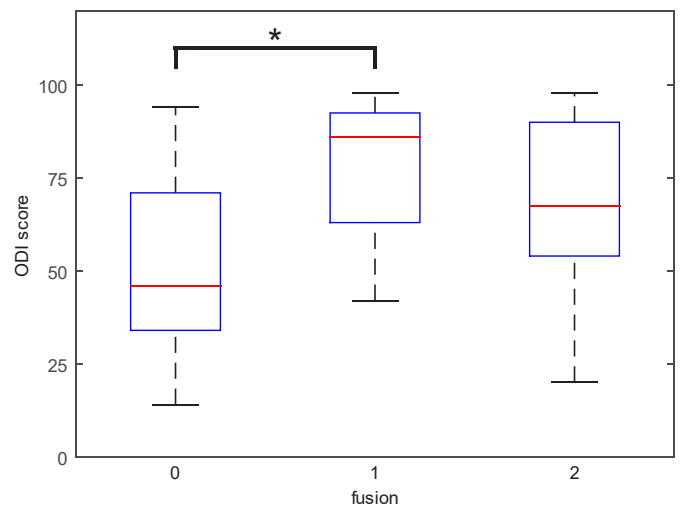

Figure 4. Boxplot to show the relation between fusion on CT (score 0, 12 ) and ODI score (0-100). The asterisk depicts a statistically significant difference. Only the difference between CT scores 0 and 1 was statistically significant.

Regarding PET scan values, Figure 5 shows the relation between the ODI category and PET endplate SUV max. Upper endplate SUV $\max U$ values were $18.1 \pm 3.8$ for ODI category 1 , $13.2 \pm 1.9$ for category 2 and $13.7 \pm 3.6$ for category 3 . The activity of the upper vertebral endplate in the entire study population was significantly higher in the lowest ODI category (with the worst clinical performance), as compared to the other ODI categories ( $p=0.02$ between ODI categories 1 and 2, $p=0.01$ between 1 and 3 and $p=0.7$ between 2 and 3 ). Lower endplate SUV $\max L$ values were $17.1 \pm 5.9$ for ODI category $1,13.5 \pm 2.1$ for category 2 and $13.7 \pm 3.6$ for category 3. For the lower endplate, similar statistically significant results were found ( $p=0.04$ between $O D I$ categories 1 and $2, p=0.05$ between 1 and 3 , $p=0.9$ between 2 and 3 ). Intervertebral disc space $S U V_{\max } D$ values were $16.8 \pm 5.0$ for ODI category $1,13.6 \pm 2.2$ for category 2 and $12.3 \pm 3.2$ for category 3 , no statistically significant differences were found between categories ( $p=0.06$ between ODI categories 1 and $2, p=0.22$ between 1 and 3 and $p=0.16$ between 2 and 3 ). For VAS and EQ-5D results were less evident, with only SUV $\max U$ scores between category 1 and 2 being significantly different $(p=0.04)$. 


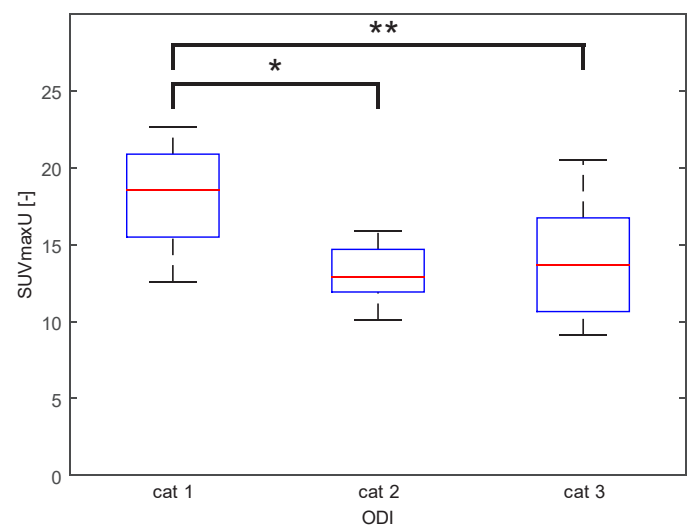

Figure 5. Boxplot to show the relation between ODI categories and SUV $\max$ endplate activity on PET. The asterisks depict statistically significant differences. $\mathrm{SUV}_{\max } \mathrm{U}$ was significantly higher in patients in ODI category 1 (with the worst clinical performance), as compared to the other categories.

SUV ratio $U$ was $1.2 \pm 0.5$ for ODI category $1,1.0 \pm 0.2$ for category 2 and $1.2 \pm 0.4$ for category 3 , differences were not statistically significant between categories. For SUV ratio, similar statistically significant results were found. Also, for VAS and EQ-5D similar statistically significant results were found. Thus, the inclusion of the activity of the intervertebral fusion area to the PET endplate activity calculation did not result in a better correlation with symptoms.

Figure 6 shows the relation between ${ }^{18} \mathrm{~F}$-fluoride PET activity, (i.e. SUV ratio $U$ and SUV ratioL), and $\mathrm{CT}$ fusion scores. As can be seen, there was a significant correlation, with $\mathrm{SUV}$ ratioU values of $1.4 \pm 0.4$ for fusion score $0,1.2 \pm 0.3$ for fusion score 1 and $1.0 \pm 0.2$ for fusion score 3 ( $p$-values between scores 0 and 1 of 0.04 ; between 0 and 2 of 0.003 ; between 1 and 2 of $p=0.09$ ). For the lower endplate similar statistically significant results were found. SUV ratio $U$ values were $1.5 \pm 0.6$ for fusion score $0,1.1 \pm 0.3$ for fusion score 1 and $1.0 \pm$ 0.3 for fusion score 3 ( $p$-values between scores 0 and 1 of 0.05 ; between 0 and 2 of 0.003 ; between 1 and 2 of $p=0.29$ ). Interestingly, the PET activity ratio of patients with fusion score 1 was not significantly lower than the PET ratio of patients with fusion score 2 . 

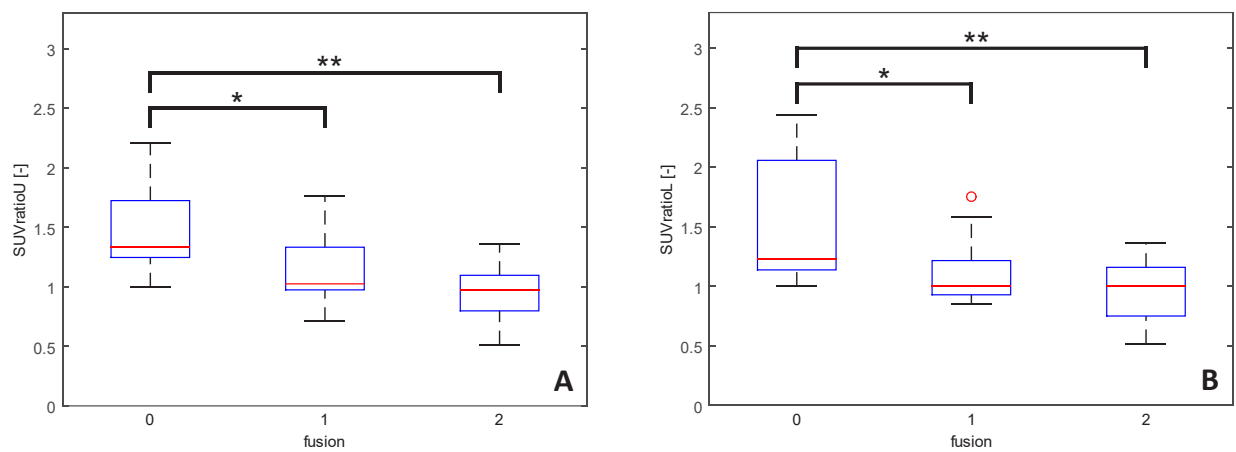

Figure 6. Boxplots to show the relation between CT fusion scores and SUV $\max$ ratios. The asterisks depict statistically significant differences. The $S U V_{\text {ratio }} U(A)$ and $S U V_{\text {ratio }} \mathrm{L}(\mathrm{B})$ in patients with pseudarthrosis (CT fusion score $0)$ were significantly higher than in patients with score 1 or 2.

\section{DISCUSSION}

This is the first study using ${ }^{18} \mathrm{~F}$-fluoride PET/CT to evaluate symptomatic as well as asymptomatic patients after spinal interbody fusion surgery and correlate findings on PET and $\mathrm{CT}$ to the patient reported functionality after the surgical procedure. Pain is often multifactorial in these patients and not always alleviated by surgery. Importantly, psychosocial factors can result in a refractory state, especially after many years of debilitating pain [7]. To minimize the impact of such confounding factors, patients were surveyed using multiple patient questionnaires simultaneously. While ODI is a specific questionnaire related to low back pain and physical limitation in daily life, VAS is a questionnaire of only subjective pain intensity, and EQ-5D relates to general health status and psychological well-being. The three questionnaire scores were similar which indicated that pain, functional capacity and psychological well-being were integratively affected in all patients. As pain is a leading determinant in decision making for surgery, correlation of imaging findings to objective clinical parameters as assessed by validated and specific patient reported outcome measures is vital.

Up until now, several studies have explored the use of ${ }^{18} \mathrm{~F}$-fluoride PET and CT in patients with persistent pain after a spinal fusion procedure. Gamie et al. [19] studied a group of 67 patients, including 25 postoperatively, with back pain and negative conventional imaging, and found in $8 / 8$ (100\%) of lumbar fusion patients positive areas of uptake in facet joint and/or disc. Fischer et al. [20] studied a group of 20 patients with persistent pain after cervical or lumbar fusion. They were able to show that even 10 years postoperatively, there was an increased tracer uptake around 8/17 of the cages in the intervertebral disc space, suggesting increased stress or micro-instability and missing or incomplete os- 
seous fusion. PET imaging supported the diagnosis of non-union or confirmed the diagnosis of complete fusion. Quon et al. [21] performed a prospective study on a cohort of 116 patients. Of these, 52/116 (45\%) were not indicated conclusively based on clinical evaluation and CT for appropriate management and therefore underwent PET/CT. Ultimately, 15 patients underwent revision surgery on the basis of abnormal ${ }^{18} \mathrm{~F}$-fluoride foci at various sites, i.e. cages, grafts, screws, rods or fixation hardware. PET/CT correctly predicted the presence of an abnormality (cage failure, screw loosening, graft fracture) requiring surgical intervention in 14 of 15 patients. Thus, PET/CT correctly identified patients requiring surgical management. Byrnes et al. [22] recently reported clinically useful ${ }^{18} \mathrm{~F}$-fluoride PET/CT findings in 49/58 (85\%) patients with neck pain after cervical fusion. While these studies indicate the value of ${ }^{18} \mathrm{~F}$-fluoride PET in a subset of symptomatic patients, they did not include pauci- or asymptomatic patients. In view of the low specificity of symptomatology and high sensitivity of the PET scan technique which carries the risk of a substantial number of false-positive cases, undesirable for surgical management, we think inclusion of pauci- or asymptomatic patients is crucial for the implementation of this technique

PET activity at the vertebral endplates ( $S U V_{\max } U$ and $S U V_{\max } L$ ) but not within the intervertebral disc space $\left(S U V_{\max } \mathrm{D}, \mathrm{SUV} \mathrm{V}_{\text {ratio }} \mathrm{U}\right.$ and $S U V_{\text {ratio }}$ ) correlated with the specific low back pain and disability symptoms as measured using ODI score. We have previously found increased tracer uptake in the vertebral endplates to be correlated with the occurrence and magnitude of subsidence in these types of patients, indicating instability with vertebral collapse of endplates [15]. Subsidence is defined as sinking of a fusion cage into one or both of the adjacent vertebral bodies [23]. However, the clinical relevance of subsidence remains under debate since the magnitude of subsidence did not match the final clinical results [24]. Fischer et al. [20] have also interpreted persistent increased uptake above and below the cage as inactive or unsuccessful fusion due to increase stress and micro-instability. We could not demonstrate a consistent correlation between ODI score and CT fusion score; while significantly different ODI scores were found between CT fusion scores 0 and 1 , this was paradoxically not the case between $\mathrm{CT}$ fusion scores 0 and 2. This could be related to the wide range of ODI scores in CT fusion score 2, which may be due to other causes of pain sensation in these patients. There appears to be an inversed correlation between the CT fusion score and SUV ratios (ratio's between SUV of both endplates and intervertebral disc space). In a normally evolving fusion the number of intervertebral bridges increases from CT fusion score 0 to 1 to 2 over time, while the PET activity develops from highly active endplates combined with a relatively silent intervertebral disc space $\left(S U V_{\text {ratio }}>1.0\right.$ ) to an even activity distribution over the two areas (SUV ratio $\approx 1.0$ ). This is evidence for the hypothesis that the existence of bony bridges ( 1 or 2 ) in combination with 'absence' of PET uptake indicate a stable fusion (the absence of pseud- 
arthrosis). And in the case of pseudarthrosis (no detectable bony bridges on CT), PET uptake pinpoints the symptomatic pseudarthrosis. Uptake at the endplates probably reflects ongoing stress reactions, due to an instable lumbar fusion.

This study was limited by a lack of correlation to the gold standard of surgical exploration. We found no previous studies on the PLIF procedure that correlated the surrogate gold standard high-resolution CT findings with surgical exploration. In posterolateral fusion, CT had a very low PPV indicating that complete mineralization of bony bridges may take substantially longer to show on CT. Prospective studies will need to address this in conjunction with PET findings. At our institution implant removal is not standard practice and revision surgery is only performed on a highly selected group of pseudarthrosis patients with substantial pain. Clinical follow-up can also be used as a surrogate gold standard, but a considerable number of patients and time intervals as well as repeated PET/CT scans would be needed to fully account for all influencing confounding factors and coexisting abnormalities. Therefore, the potential of therapy management prediction by the PET scan could not be fully assessed in this study. However, the main goal of this study was to evaluate the relationship between active PET scan abnormalities and symptoms. Furthermore, large differences in time interval between fusion surgery and ${ }^{18} \mathrm{~F}$-fluoride PET/CT examination were present between patients. A similar follow-up period for all patients would have been ideal, but was not feasible within the limits of clinical practice, because symptomatic patients can present with pain at any time from months to years after PLIF. For asymptomatic patients, it is not feasible to perform PET/CT many months to years after a successful fusion procedure. Finally, another limitation of the study was the small sample size.

In conclusion, our study demonstrates that PET uptake shows superior correlation to symptomatic pseudarthrosis as compared to CT. Furthermore, an inversed relationship between PET uptake and CT score was found. 


\section{REFERENCES}

1. van Tulder MW, Koes BW, Bouter LM. A cost-of-illness study of back pain in The Netherlands. Pain. 1995; $62(2): 233-240$.

2. Walker BF. The prevalence of low back pain: a systematic review of the literature from 1966 to 1998. Journal of spinal disorders. 2000; 13(3):205-217.

3. Lambeek LC, van Tulder MW, Swinkels IC, Koppes LL, Anema JR, van Mechelen W. The trend in total cost of back pain in The Netherlands in the period 2002 to 2007. Spine. 2011; 36(13):1050-1058.

4. Deyo RA, Bass JE, Walsh NE, Schoenfeld LS, Ramamurthy S. Prognostic variability among chronic pain patients: implications for study design, interpretation, and reporting. Archives of physical medicine and rehabilitation. 1988; 69(3 Pt 1):174-178.

5. Willems PC, Staal JB, Walenkamp GH, de Bie RA. Spinal fusion for chronic low back pain: systematic review on the accuracy of tests for patient selection. The spine journal : official journal of the North American Spine Society. 2013; 13(2):99-109.

6. Raizman NM, O'Brien JR, Poehling-Monaghan KL, Yu WD. Pseudarthrosis of the spine. The Journal of the American Academy of Orthopaedic Surgeons. 2009; 17(8):494-503.

7. Larsen JM, Capen DA. Pseudarthrosis of the Lumbar Spine. The Journal of the American Academy of Orthopaedic Surgeons. 1997; 5(3):153-162.

8. Turner JA, Ersek M, Herron L, Haselkorn J, Kent D, Ciol MA, et al. Patient outcomes after lumbar spinal fusions. JAMA : the journal of the American Medical Association. 1992; 268(7):907-911.

9. Zanoli G, Stromqvist B, Jonsson B. Visual analog scales for interpretation of back and leg pain intensity in patients operated for degenerative lumbar spine disorders. Spine. 2001; 26(21):2375-2380.

10. Willems P. Decision making in surgical treatment of chronic low back pain: the performance of prognostic tests to select patients for lumbar spinal fusion. Acta orthopaedica Supplementum. 2013; 84(349):1-35.

11. Brodsky AE, Kovalsky ES, Khalil MA. Correlation of radiologic assessment of lumbar spine fusions with surgical exploration. Spine. 1991; 16(6 Suppl):S261-265.

12. Carreon LY, Djurasovic M, Glassman SD, Sailer P. Diagnostic accuracy and reliability of fine-cut CT scans with reconstructions to determine the status of an instrumented posterolateral fusion with surgical exploration as reference standard. Spine. 2007; 32(8):892-895.

13. Hilibrand AS, Dina TS. The use of diagnostic imaging to assess spinal arthrodesis. The Orthopedic clinics of North America. 1998; 29(4):591-601.

14. van Tulder MW, Koes BW, Bouter LM, Metsemakers JF. Management of chronic nonspecific low back pain in primary care: a descriptive study. Spine. 1997; 22(1):76-82.

15. Brans B, Weijers R, Halders S, Wierts R, Peters M, Punt I, et al. Assessment of bone graft incorporation by $18 \mathrm{~F}$-fluoride positron-emission tomography/computed tomography in patients with persisting symptoms after posterior lumbar interbody fusion. EJNMMI research. 2012; 2(1):42.

16. Fairbank JC, Pynsent PB. The Oswestry Disability Index. Spine. 2000; 25(22):2940-2952; discussion 2952.

17. Fairbank JC, Couper J, Davies JB, O'Brien JP. The Oswestry low back pain disability questionnaire. Physiotherapy. 1980; 66(8):271-273. 
18. Lamers LM, Stalmeier PF, McDonnell J, Krabbe PF, van Busschbach JJ. [Measuring the quality of life in economic evaluations: the Dutch EQ-5D tariff]. Nederlands tijdschrift voor geneeskunde. 2005; 149(28):1574-1578.

19. Gamie S, El-Maghraby T. The role of PET/CT in evaluation of Facet and Disc abnormalities in patients with low back pain using (18)F-Fluoride. Nuclear medicine review Central \& Eastern Europe. 2008; 11(1):1721.

20. Fischer DR, Zweifel K, Treyer V, Hesselmann R, Johayem A, Stumpe KD, et al. Assessment of successful incorporation of cages after cervical or lumbar intercorporal fusion with [(18)F]fluoride positron-emission tomography/computed tomography. European spine journal : official publication of the European Spine Society, the European Spinal Deformity Society, and the European Section of the Cervical Spine Research Society. 2011; 20(4):640-648.

21. Quon A, Dodd R, lagaru A, de Abreu MR, Hennemann S, Alves Neto JM, et al. Initial investigation of (1)(8)F$\mathrm{NaF}$ PET/CT for identification of vertebral sites amenable to surgical revision after spinal fusion surgery. European journal of nuclear medicine and molecular imaging. 2012; 39(11):1737-1744.

22. Byrnes TJ, Xie W, Al-Mukhailed O, D'Sa A, Novruzov F, Casey AT, et al. Evaluation of neck pain with (18)F$\mathrm{NaF}$ PET/CT. Nuclear medicine communications. 2014; 35(3):298-302.

23. Williams AL, Gornet MF, Burkus JK. CT evaluation of lumbar interbody fusion: current concepts. AJNR American journal of neuroradiology. 2005; 26(8):2057-2066.

24. Tokuhashi Y, Ajiro Y, Umezawa N. Subsidence of metal interbody cage after posterior lumbar interbody fusion with pedicle screw fixation. Orthopedics. 2009; 32(4). 



\section{CHAPTER 4}

${ }^{18} \mathrm{~F}$-fluoride $\mathrm{PET} / \mathrm{CT}$ to identify pain generators after lumbar spinal fusion -

a retrospective analysis of symptomatic and asymptomatic patients

Marloes JM Peters, Boudewijn T Brans, Wouter AM Broos, Elisabeth MC Jutten, Mariëlle GW Visser, Felix M Mottaghy, Olaf Schijns, René E Weijers, Paul C Willems 


\section{ABSTRACT}

\section{OBJECT}

Identification of the cause and mechanism of persistent or recurrent pain after lumbar spinal fusion is essential in delineating the optimal treatment for the patient. The aim of this study was to follow-up symptomatic and asymptomatic patients after posterior lumbar interbody fusion (PLIF) by means of ${ }^{18} \mathrm{~F}$-fluoride positron emission tomography (PET)/CT and patients reported outcome measures (PROMs). Both CT and PET/CT findings were correlated to PROMs to assess which findings could be considered possible pain indicators.

\section{METHODS}

A cohort of 36 PLIF patients that underwent ${ }^{18} \mathrm{~F}$-fluoride PET/CT scanning was included. Sixty minutes after intravenous injection of 100-248 MBq (mean 194.4 MBq; median 196.5 MBq) ${ }^{18} \mathrm{~F}$-fluoride, PET and CT images were acquired. Interbody bony fusion, subsidence, screw loosening and facet joint status were scored on CT. Bone metabolism activity of the endplates, intervertebral disc space, screws and facet joints were quantified on PET/CT. The amount of pain and disability was quantified by means of three validated PROMs. Patients were categorized based on their PROM scores and based on their CT scores. Differences between categories were assessed. Bone metabolism values of asymptomatic patients were averaged to obtain a map with typical bone metabolism values of spinal structures after PLIF.

\section{RESULTS}

Symptomatic patients appeared to be characterized by abnormal PET/CT values at the lower endplates, facet joints and/or screws. No significant differences in PROMs between the CT categories were found. Typically, asymptomatic patients after PLIF showed 1. high activity at the operated endplates as compared to adjacent non-operated levels; 2 . similar values in the intervertebral disc space and at the endplates of the operated segment; 3. moderately elevated values of the facet joints one level above the operated segment; and 4. moderate bone metabolism values surrounding the screws. Values outside this "normal" range could point to a pain indicator.

\section{CONCLUSIONS}

Findings on PET/CT correlated better with PROMs compared to findings on $\mathrm{CT}$. When interpreting ${ }^{18} \mathrm{~F}$-fluoride PET/CT scans of symptomatic patients after PLIF, it is important to realize that bone metabolism patterns of spinal structures in and around the operated segment might change inherent to the surgical procedure performed. Therefore, not every hotspot on ${ }^{18} \mathrm{~F}$-fluoride PET/CT should be attributed as pain generator regardless. Future studies should demonstrate whether treatments based on ${ }^{18} \mathrm{~F}$-fluoride PET/CT findings would lead to more benefit for the patient. 


\section{INTRODUCTION}

Spinal fusion is a surgical procedure in which fixation of vertebral segments is achieved by means of instrumentation and bone grafting to treat patients suffering from back pain. Pain relief is achieved by restoration of the height of the segment, decompression of the nerve roots and definite bony fusion of the vertebrae involved. However, 5 to $50 \%$ of patients will suffer from persistent or recurrent back and/or leg pain after spinal surgery [1]. Identification of the cause and mechanism of pain months to years after spinal fusion is essential in delineating the treatment path that will be most beneficial for the patient. The cause of pain in these patients can be multifactorial [2] and pain after spinal fusion is not always related to the surgical procedure that was performed [3], which complicates diagnosis and treatment. The patients' symptoms can be evaluated by clinical examination and quantified by patient reported outcome measures (PROMs). Imaging however, is required to identify the cause and mechanism of pain [2], especially before going over to invasive treatments, to make sure that the treatment of choice is likely to solve the problem that causes the pain.

Imaging modalities currently used for patients with persistent pain after spinal fusion, include plain radiographs to detect failure of instrumentation, magnetic resonance imaging (MRI) to detect root nerve compression and computed tomography (CT) to confirm and/or exclude anatomical abnormalities such as pseudarthrosis, subsidence and failure of instrumentation. However, these findings do not always correlate with the patient's symptoms directly [3-5]. Alternatively, nuclear based imaging modalities can be used to diagnose symptomatic spinal fusion patients by assessment of abnormalities in bone metabolism.

Several studies have used ${ }^{18} \mathrm{~F}$-fluoride positron emission tomography (PET) /CT to evaluate symptomatic spinal fusion patients [6-12]. PET/CT with the bone-seeking tracer ${ }^{18} \mathrm{~F}$ fluoride allows localization and quantification of bone metabolism and one of its indications is bone pain [13]. Good diagnostic sensitivity of ${ }^{18}$ F-fluoride PET/CT as diagnostic tool has been shown in patients with back pain [14-16] and otherwise unexplained bone pain [17-20]. Regarding diagnostic specificity, changes in bone metabolism inherent to the surgical procedure performed need to be distinguished from hotspots related to symptoms. For example, ongoing bone repair mechanisms years after successful surgery [21] and/or redistribution of mechanical loads which occur particularly in the adjacent segments [22], will both be reflected by alterations in bone metabolism but are not necessarily related to symptoms. It is therefore important to analyze asymptomatic patients as well as symptomatic patients.

The aim of this study was to follow-up symptomatic as well as asymptomatic patients after posterior lumbar interbody fusion (PLIF) by means of ${ }^{18} \mathrm{~F}$-fluoride PET/CT and 
PROMs. Abnormalities of spinal structures of the operated segment and adjacent vertebrae were evaluated on CT and on PET/CT. Both CT and PET/CT findings were correlated to PROMs to assess which findings could be considered as possible pain indicators. A pool of asymptomatic patients was used to assess changes in bone metabolism that were not necessarily related to symptoms but could be assumed inherent to the surgical procedure that was performed.

\section{METHODS}

\section{Patients}

A total of 40 patients who had undergone PLIF with a postoperative PET/CT scan between June 2008 and April 2015 were considered retrospectively eligible for inclusion. Of these 40 eligible patients, 36 patients were willing to fill out questionnaires and were subsequently included in the study. Sixteen of the included patients suffered from persistent or recurrent low back pain without an apparent cause on conventional imaging. These patients underwent PET/CT scanning as an additional diagnostic examination at a variable time point after surgery. The other twenty patients were recruited from a consecutive cohort during routine clinical and radiological (radiographs and CT) investigation one year after PLIF and underwent PET/CT scanning as part of the study protocol. PLIF was performed as described before $[9,23]$. Three patients had undergone PLIF surgery at two levels. Operated levels were L3-L4 ( $n=3), \mathrm{L} 4-\mathrm{L} 5(n=15)$ and L5-S1 $(n=21)$. PET/CT scanning was performed at an average of 18.8 months (range 9 to 76 months, median 12.5 months) after PLIF. This study was performed in accordance with the Helsinki Declaration of 1975, as revised in 2013, and the protocol was accepted by the medical ethical committee of the Maastricht University Medical Center (NL.32881.068.11) in which patients gave their written informed consent.

\section{${ }^{18}$ F-fluoride PET/CT scan acquisition}

Sixty minutes after intravenous injection of 100-248 MBq (mean 194.4 MBq; median $196.5 \mathrm{MBq}){ }^{18} \mathrm{~F}$-fluoride, PET and CT images were acquired with an integrated PET/CT scanner (Gemini TF 64 PET-CT, Philips, the Netherlands). After a low-dose CT acquisition (120 kV, 30 mAs, slice thickness $4 \mathrm{~mm}$ ) covering the whole lumbosacral spine, a static PET scan of two bed positions of five minutes each was acquired. This was immediately followed by a diagnostic CT scan (64-slice helical, 120 kV, 250 mAs, slice thickness $1 \mathrm{~mm}$, increment $0.8 \mathrm{~mm}$ ). Scans were viewed on clinical software (EBW, Philips, the Netherlands) and further analyzed on a research tool (PMOD 3.0, PMOD Technologies Ltd, Zürich). 


\section{Diagnostic CT scan analysis}

Evaluation of the CT scans was performed independently by three blinded observers; an experienced musculoskeletal radiologist (RW), an experienced nuclear medicine physician (BB) and a junior nuclear medicine physician (WB). Discrepancies between observers were resolved by consensus. Interbody fusion (CT_FUSION) was scored within each operated segment as either 0,1 or 2, according to the number of bony bridges between the vertebrae (Figure 1A), as described before [9]. A bony bridge was defined as bone mass progressing from one endplate to the other without interruptions. For patients that underwent PLIF at two segments, the lowest fusion score of the operated segments (worstcase) was used as fusion score for that patient. Subsidence (CT_SUBS) of the intervertebral cages into the endplates was scored as 0,1 or 2 according to the severity of subsidence (Figure 1B), as described before [9]. An imaginary line was drawn following the original endplate outline, from which the degree of subsidence into the vertebrae was scored. This amounted to a subsidence score for the upper and lower endplate per operated segment. The maximum subsidence score (worst-case) per patient (CT_SUBS) was used for further analysis. Screw loosening (CT_SCREW) was defined as the presence of radiolucent lines surrounding the screws. Each screw was scored as either 0 (no loosening) or 1 (loosening) (Figure 1C). If one of the screws within a patient was reported as loosened, screw loosening was reported to be present for that particular patient. The status of the facet joints (CT_FACET) was scored for the facet joints of the two levels above the operated segment as either 1 (normal) or 2 (degenerated) (Figure 1D). The facet joints of the operated segments were removed during surgery to access the intervertebral disc space. When one of the facet joints within a patient was scored as degenerated, facet joint degeneration was reported to be present for that particular patient. 


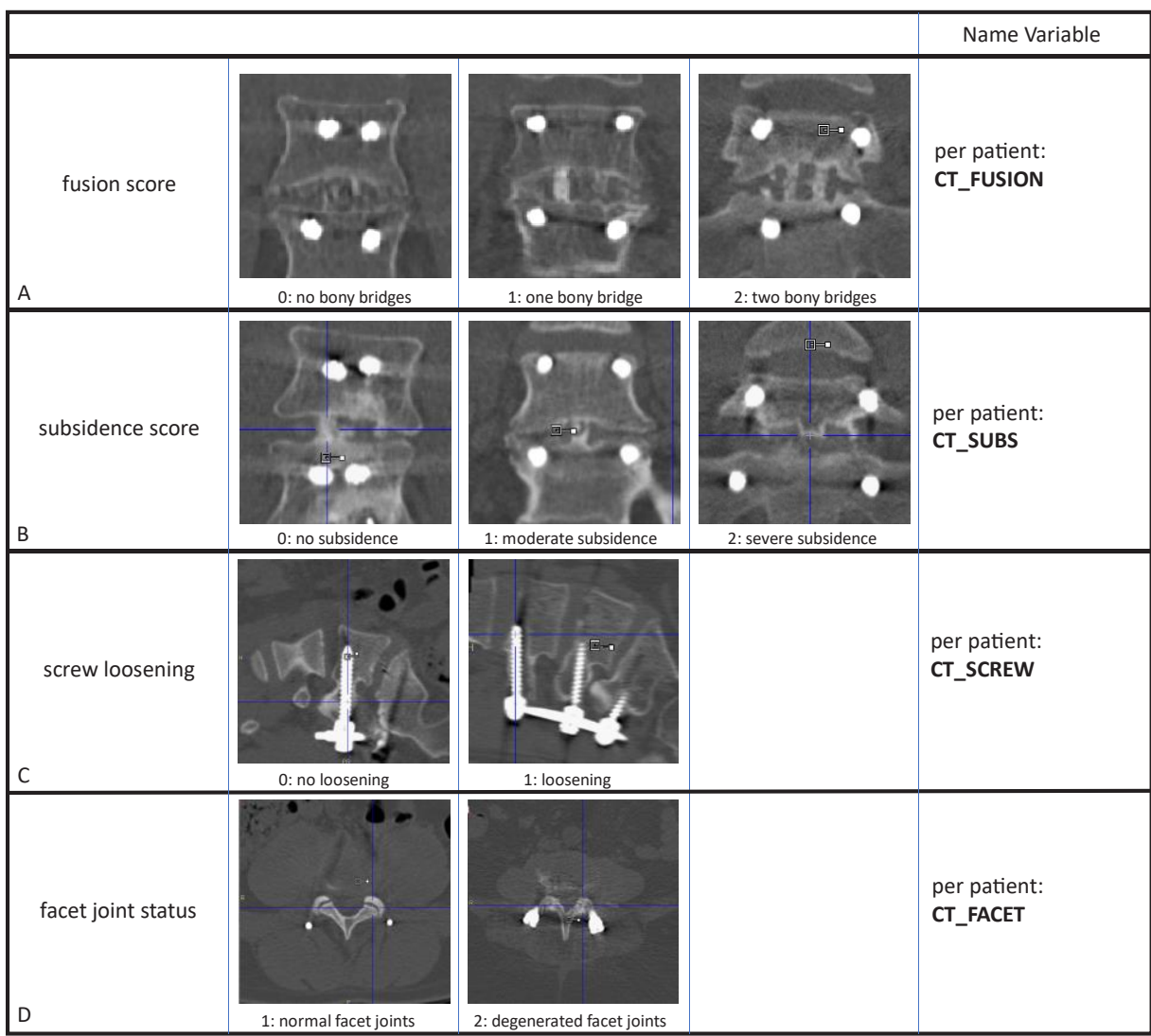

Figure 1. Examples of scoring on CT, including the names of the variables per patient. Fusion (CT_FUSION) was scored as either 0 (no bony bridges), 1 (one bony bridge) or 2 (two or more bony bridges) (A). Subsidence (CT_SUBS) was scored as 0 (no subsidence), 1 (moderate subsidence) or 2 (severe subsidence) (B). Screw loosening (CT_SCREW) was scored as 0 (no loosening) or 1 (loosening) (C). Facet joint status (CT_FACET) was scored as 1 (normal) or 2 (degenerated) (D).

\section{${ }^{18}$ F-fluoride PET/CT scan analysis}

The ${ }^{18} \mathrm{~F}$-fluoride PET/CT scans were evaluated by three independent, blinded observers (MP, BB, WB). Discrepancies between observers were resolved by consensus. Several volumes of interest (VOIs) were drawn in the low-dose CT image and transferred to the coregistered PET image to calculate maximum standardized uptake values (SUV $\max$ ) of ${ }^{18} \mathrm{~F}$ fluoride as measure of bone metabolism. SUV was calculated by dividing the measured ${ }^{18}$ F-fluoride activity in a region by the injected dose of the tracer and the mass of the patient. Bone metabolic activity at the endplates and in the intervertebral disc space was calculated for each operated segment. Three ellipsoid shaped VOIs were manually drawn in each operated segment on low-dose CT following the contours of the vertebrae (slice 
thickness $4 \mathrm{~mm}$, short axis range: $40-50 \mathrm{~mm}$, long axis range: $55-65 \mathrm{~mm}$ ) to obtain SUV $\max$ at the upper endplate (SUV max_endUP), lower endplate (SUV max_endLOW) and the intervertebral disc space (SUV max inter) (Figure 2A) as before [9]. In patients that underwent PLIF at two segments, the maximum value per patient were used. Bone metabolic activity of the screw surroundings was calculated by drawing a rectangular beam (with dimensions $50 \mathrm{~mm} \times 20 \mathrm{~mm} \times 20 \mathrm{~mm}$ ) around each screw on low-dose CT (Figure 2B). The maximum screw activity within a patient (SUV max_screw) was used for further analyses. The facet joint bone metabolic activity of each facet joint within the field of view was calculated. Spheres with a diameter of $10 \mathrm{~mm}$ were drawn around each facet joint on CT to determine the SUV $\max$ per facet joint (Figure $2 \mathrm{C}$ ). The facet joint with the highest SUV $\max$ per patient was used for further analysis (SUV max facet).

\begin{tabular}{|l|c|c|c|c|}
\hline & CT & Name Variable \\
\hline $\begin{array}{c}\text { endplate activity } \\
\text { intervertebral activity }\end{array}$ & & & & \\
\hline
\end{tabular}

Figure 2. Examples of the VOI based approach to obtain SUV $\mathrm{max}_{\text {ax }}$ of the different spinal structures, including the names of the variables per patient. VOIs were drawn on the low-dose CT image and transferred to the coregistered PET image to calculate the SUV $V_{\max }$. Three ellipsoid VOIs were drawn at the endplates and interverte-

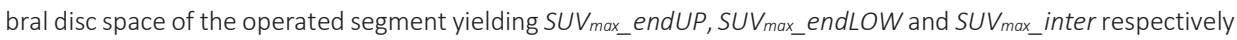
(A). Rectangular beams were drawn around each screw. The screw with the highest SUV $\max$ per patient was used for further analysis (SUV max_screw) (B). Spheres were drawn around each facet joint. The facet joint with the highest SUV $\max$ per patient was used for further analysis (SUV max facet) (C). 
Patient reported outcome measures (PROMs)

The amount of pain and disability per patient was quantified by means of three validated questionnaires that are commonly used in patients with back pain:

The Oswestry Disability Index (ODI) evaluates ten general daily tasks based on back related function [24] and can be used to assess functional outcome in patients treated for low-back pain [25]. Back pain and/or leg pain was quantified by the Visual Analogue Scale (VAS) [26]. Patients were asked to express the amount of pain in their back, right and left leg ranging from 0 (no pain) to 100 (worst pain). The score for back pain was used as measure in this study. The EuroQol (EQ)-5D measures health-related quality of life in five domains (i.e. mobility, self-care, usual activities, pain/discomfort and anxiety/depression) [27]. The EQ-5D index score was calculated based on a Dutch value set, representative of the Dutch population with regards to age and gender [28].

To facilitate further analysis of the data, each questionnaire score was linearly re-scaled from 0 (worst possible score) to 100 (best possible score). Patients were divided into categories based on their re-scaled questionnaire score; patients with scores 0-40 were classified into category 1 ("worst score"), scores 40-60 into category 2 ("intermediate score"), and scores 60-100 into category 3 ("best score") respectively. Patients were considered asymptomatic if at least two of the three questionnaire scores were above 80 and all three questionnaire scores were above 60 . Bone metabolism values of the patients that were considered asymptomatic, were averaged for each spinal structure to obtain a map with typical bone metabolism values of spinal structures after spinal fusion surgery.

\section{Statistical analysis}

Statistical evaluation was performed using IBM SPSS Statistics 23.0 (Armonk, NY: IBM Corporation). The 36 patients were divided into categories based on their PROM scores and based on their CT scores. Differences between these categories were assessed by an independent t-test in case of normality and by a Mann-Whitney U-test (two categories) or a Kruskall-Wallis test (more than two categories) otherwise. In case the Kruskall-Wallis test indicated significant differences between categories, the Mann-Whitney U-test was used to specify the categories between which the significant difference was present. Pvalues $\leq 0.05$ were used to indicate a statistically significant difference. 


\section{RESULTS}

CT findings in relation to PROMS

The 36 patients were divided into categories according to the scores of the four CT parameters (fusion, subsidence, screw loosening and facet joint status). Table 1 shows the mean values of the PROM questionnaires per CT category. No significant differences in PROMs between the categories of the CT parameters were found.

${ }^{18}$ F-fluoride PET/CT findings in relation to PROMs

The 36 patients were subsequently divided into categories based on their PROM scores (ODI, VAS and EQ-5D). Table 2 shows the mean values of the bone metabolic activity at the upper and lower endplate, intervertebral disc space, screws and facet joints per PROM category. Significant differences between PROM categories were found in the bone metabolic activity of the lower endplate, screw surroundings and facet joints. The significant differences are illustrated in Figure 3. Patients in ODI category 1 (worst category) showed higher bone metabolism values at the lower endplate, at the facet joints and at the screw surroundings compared with patients in the better ODI categories 2 and 3. This was similar for the EQ-5D questionnaire. Pain on VAS correlated with bone metabolic activity surrounding the screws. 


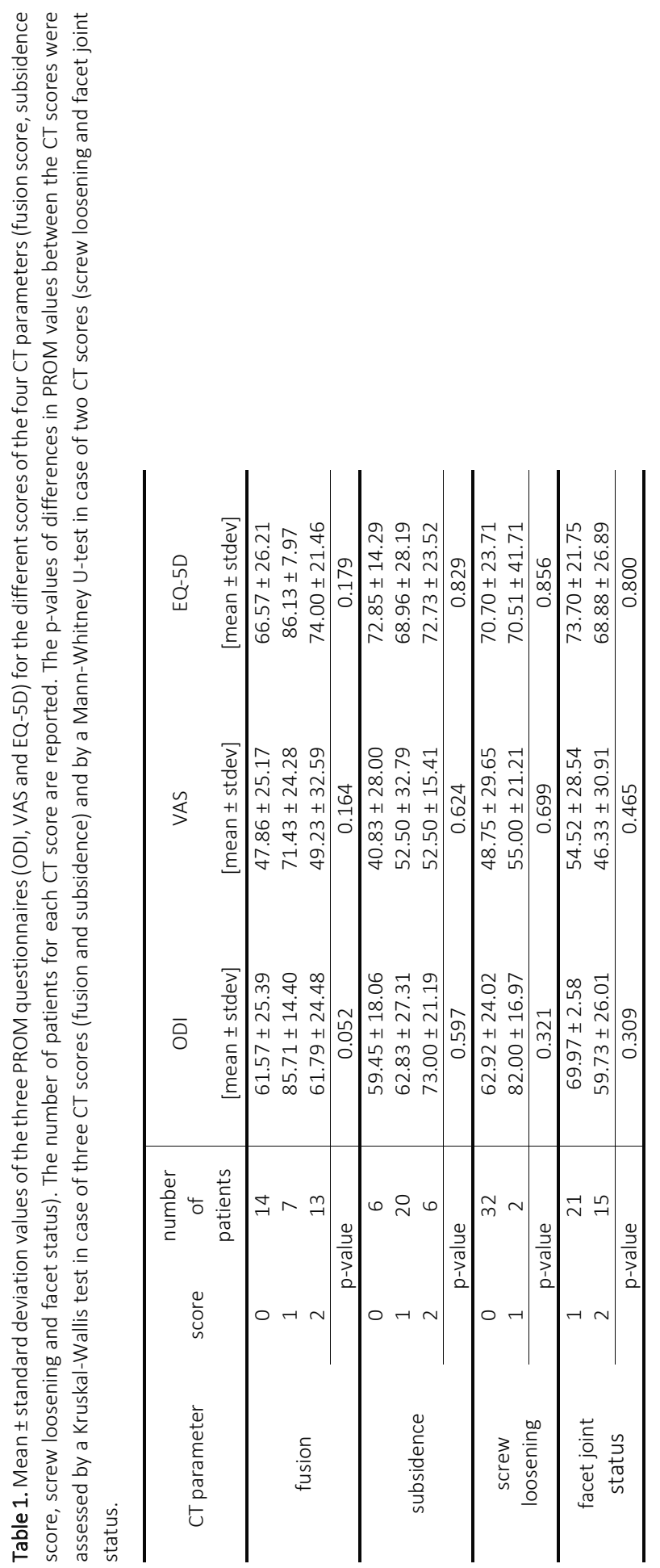




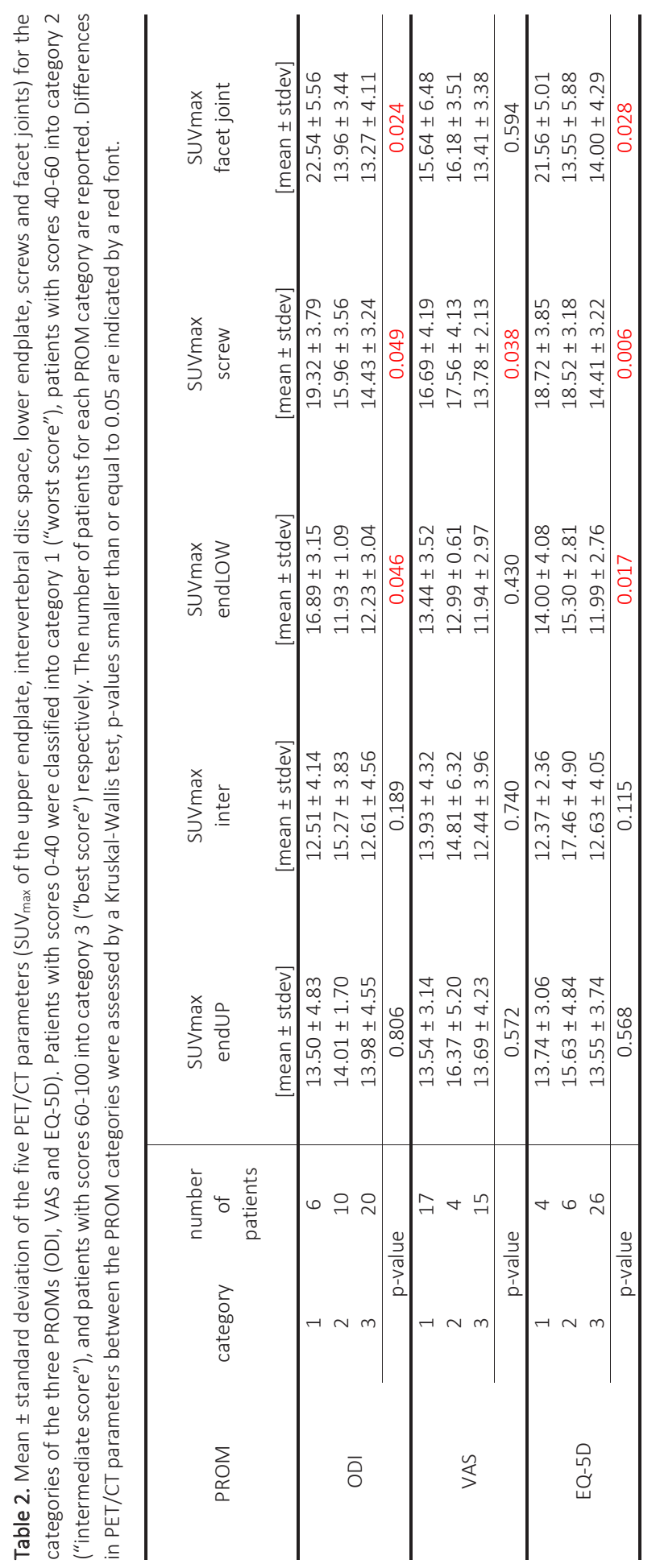




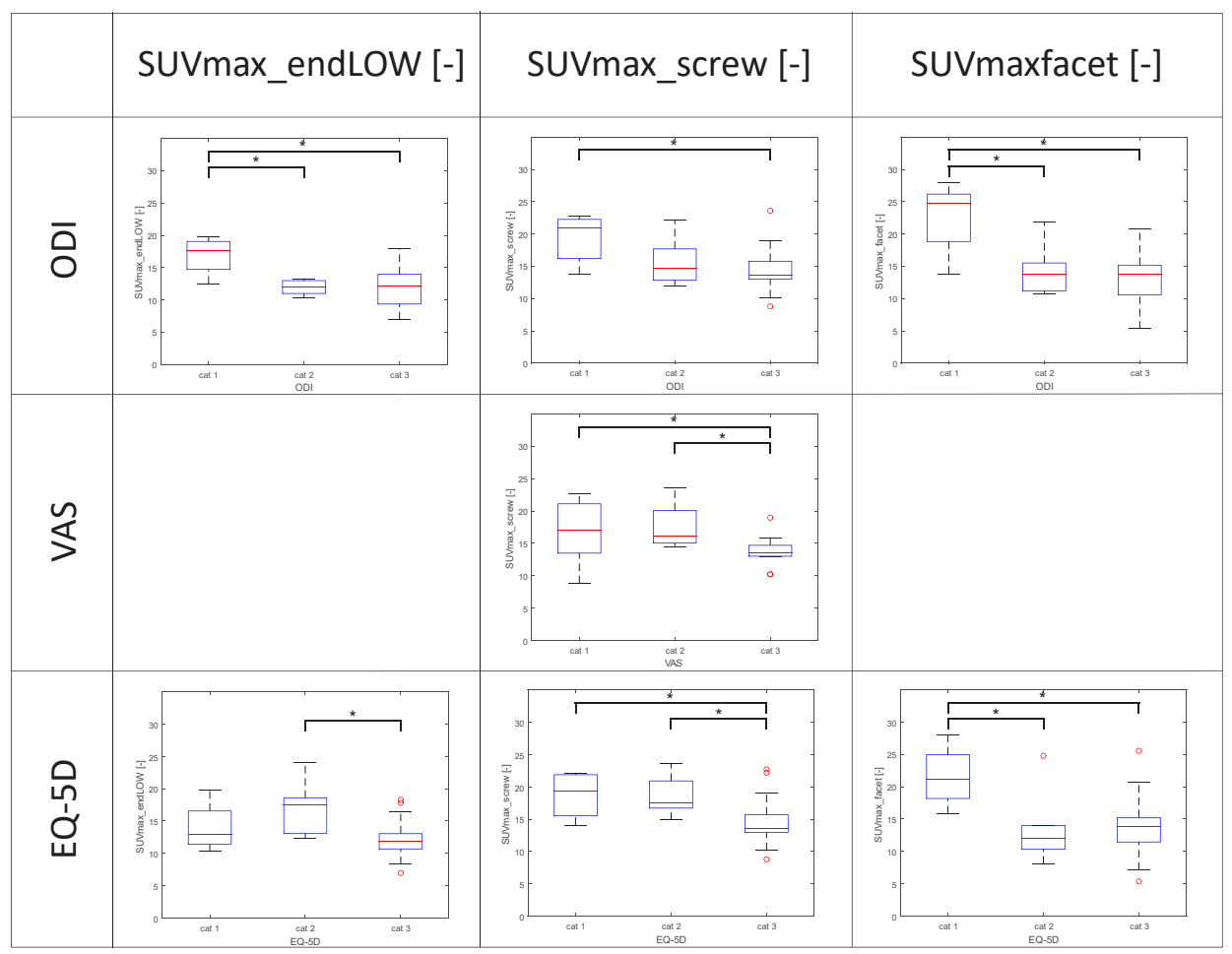

Figure 3. Illustrations of the significant correlations between PROMs and PET/CT parameters. Patients were divided into categories based on their questionnaire score: patients with scores 0-40 were classified into category 1 ("worst score"), patients with scores 40-60 into category 2 ("intermediate score"), and patients with scores 60-100 into category 3 ("best score") respectively. Asterisks represent statistically significant differences in PET/CT parameters between patients in different PROM categories $(p \leq 0.05)$.

\section{Correlation between ${ }^{18}$ F-fluoride PET/CT and CT findings}

To evaluate the coherence between anatomical findings on CT and bone metabolism findings on PET/CT, CT and PET/CT parameters were correlated to each other. The 36 patients were divided into categories based on the scores of the four CT parameters (fusion, subsidence, screw loosening and facet joint status). Table 3 shows the distribution of bone metabolic activity (mean \pm standard deviation) of the upper and lower endplate, intervertebral disc space, screw surroundings and facet joints per CT category. P-values are reported in Table 3. A grid of figures correlating each CT score to each PET/CT parameter is provided in the appendix (Figure 4). Bone metabolism activity of both endplates on $\mathrm{PET} / \mathrm{CT}$ was inversely proportional to the amount of bony bridges on CT. The amount of subsidence on CT was related to the level of bone metabolic activity of the lower end plate and of the facet joints. Also, degenerated facet joints showed higher bone metabolic activity compared to normal facet joints. 


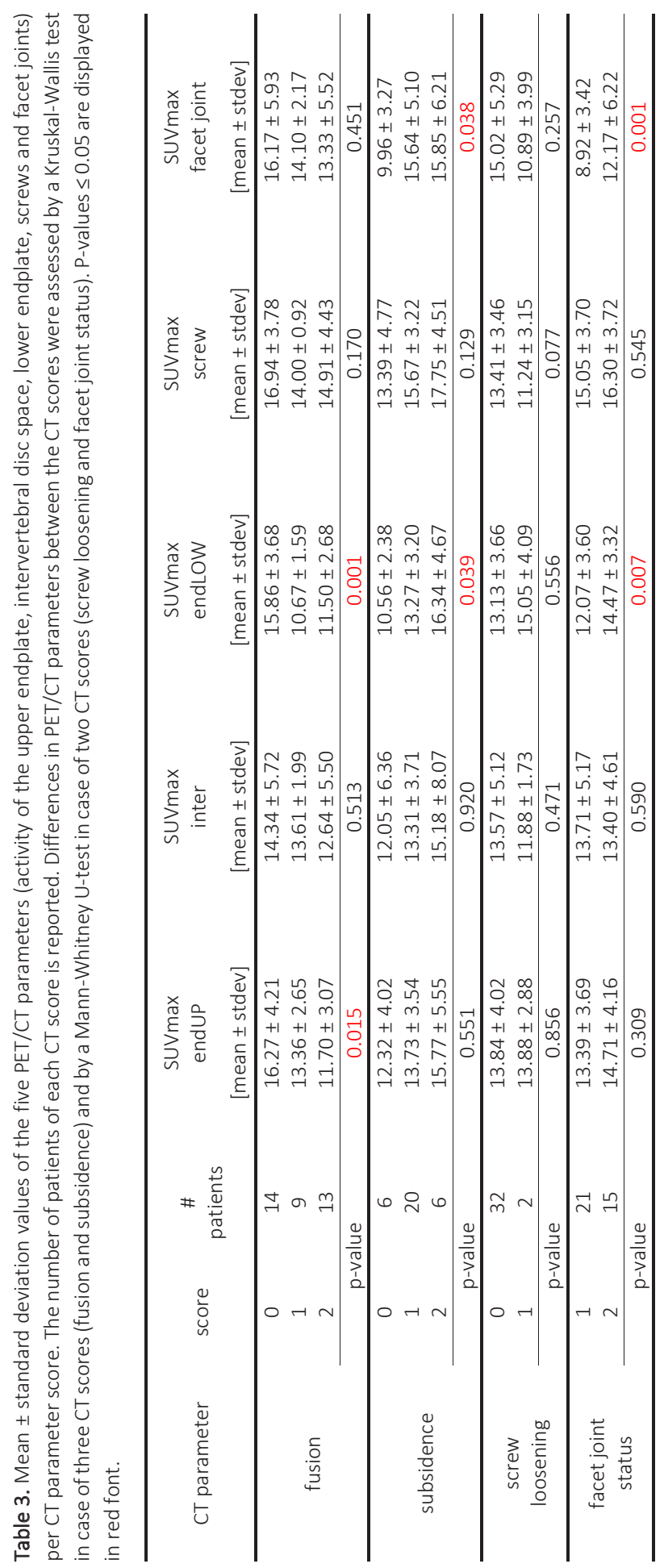




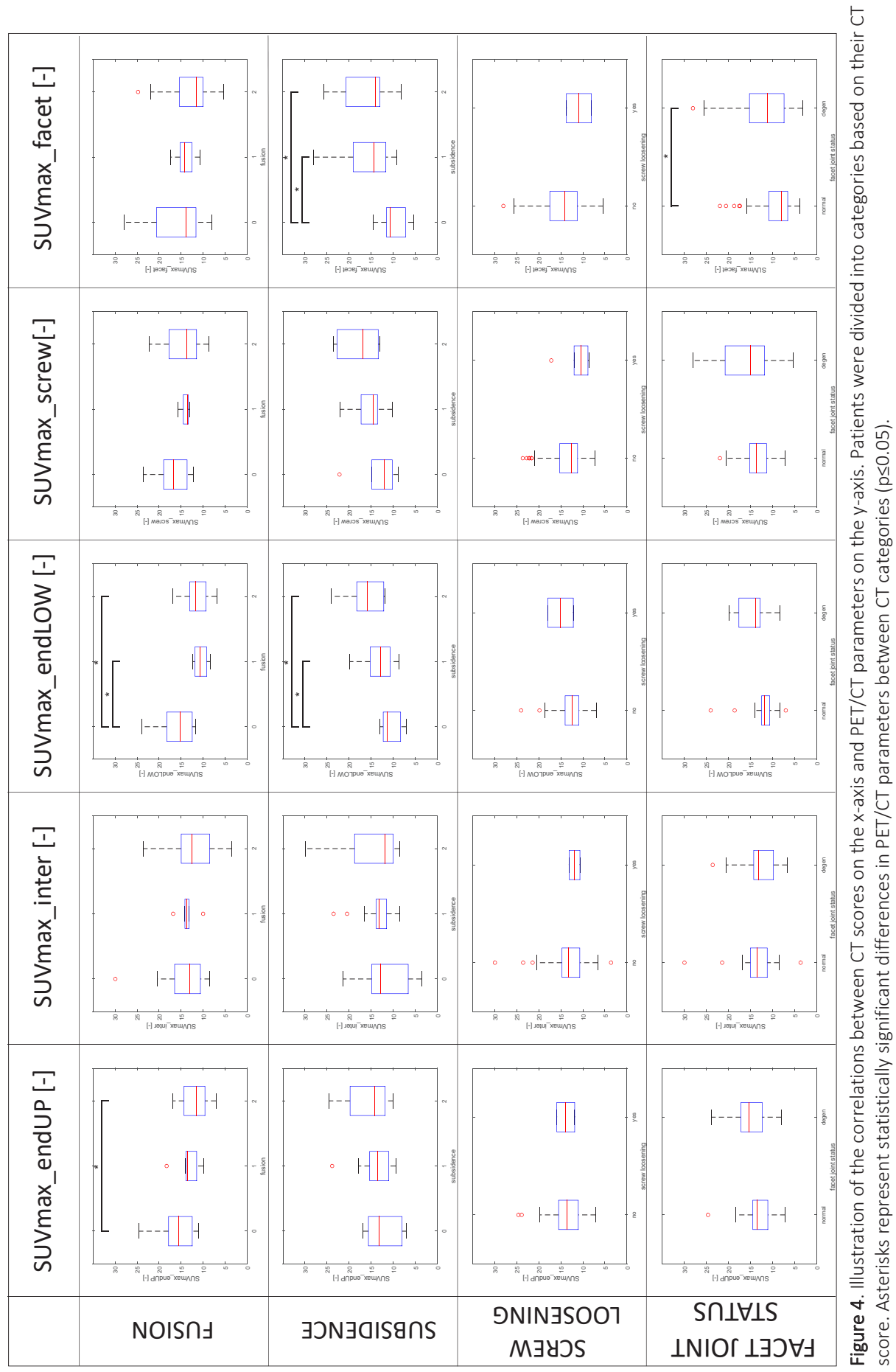


Asymptomatic bone metabolism profile after spinal fusion surgery

Fourteen patients were considered asymptomatic according to the criteria specified in the Methods section. Bone metabolic values of these patients were averaged for each spinal structure to obtain a "normal" bone metabolism map of the spine after spinal fusion (Figure 5). Typically, asymptomatic patients after spinal fusion showed 1. high activity at the endplates of the operated segment as compared to adjacent non-operated levels; 2. similar SUV $\max$ in the intervertebral disc space and at the endplates in the operated segment, while non-operated levels show higher activity at the endplates as compared to intervertebral; 3. moderately elevated values of the facet joints one level above the operated segment; and 4. only moderate bone metabolism values surrounding the screws.
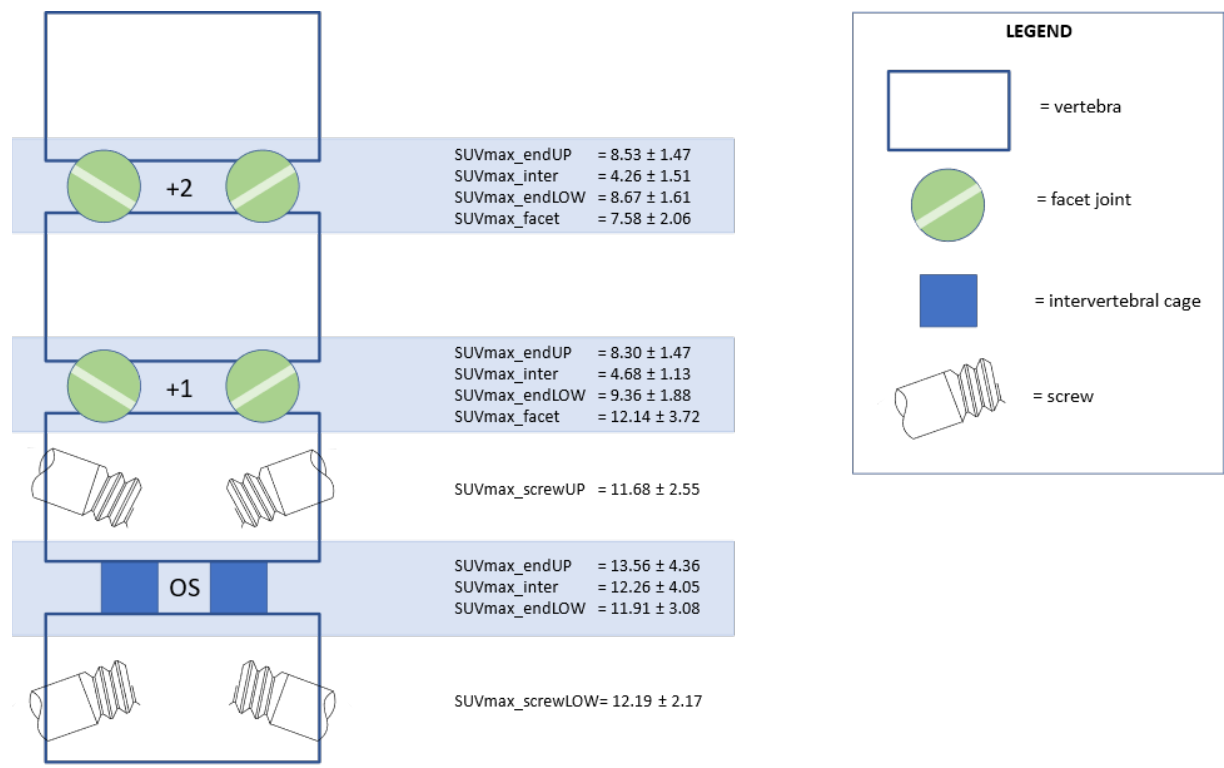

Figure 5. Schematic drawing of the spinal column after spinal fusion. The bottom segment resembles the operated segment (OS) including the pedicle screws. Two adjacent vertebrae with their facet joints are also represented. The mean bone metabolism values \pm standard deviation of several spinal structures of the fourteen asymptomatic patients are noted besides the picture. The legend explains the drawings that were used for each spinal structure. 


\section{DISCUSSION}

The most important finding of this study is that ${ }^{18} \mathrm{~F}$-fluoride PET/CT parameters correlated better to the clinical status (PROMs) in patients after spinal fusion than CT parameters. Patients with poor questionnaire scores, showed higher bone metabolism values at the lower endplate, at the facet joints and surrounding the screws. Not all increased radiotracer uptake should be attributed as pain generators regardless, since asymptomatic patients typically showed 1 . higher activity at the endplates of the operated segment as compared to adjacent non-operated levels; 2 . similarly high SUV $\max$ in the intervertebral disc space and at the endplates in the operated segment; 3. moderately elevated values of the facet joints one level above the operated segment; and 4. moderate bone metabolism values surrounding the screws. These changes in bone metabolism patterns could be considered inherent to the spinal fusion procedure that was performed. Another finding of this study was the functional interaction of the different spinal structures as a possible source of perceived pain and disability after spinal fusion, using the ${ }^{18} \mathrm{~F}$-fluoride $\mathrm{PET} / \mathrm{CT}$ technique. Symptomatic patients were characterized by abnormal PET/CT values at the lower endplates, facet joints and screw surroundings. This could be an illustration of the redistribution of mechanical load in a more complicated course. In asymptomatic patients, higher values to a limited extent may be seen in adjacent levels such as the facet joints, as a result of transferred stresses (so-called "adjacent level stress"). The transfer of stresses was also illustrated by the occurrence of subsidence on $\mathrm{CT}$, which was shown to correlate to higher metabolic activity of the lower endplate on PET/CT. This implies that subsidence gives rise to additional bone stress at the endplates. Subsidence also correlated with higher facet joint activity on PET/CT. By lowering the height of the operated segment, subsidence probably increases tension on the adjacent facet joints which was reflected by higher facet joint activity values on PET/CT.

Several studies have explored the use of ${ }^{18} \mathrm{~F}$-fluoride PET/CT in patients with persistent pain after lumbar spinal fusion without obvious clinical explanation and/or negative conventional imaging, previously. Gamie et al. [8] studied eight lumbar fusion patients with back pain postoperatively and negative conventional imaging. They analyzed ${ }^{18} \mathrm{~F}$-fluoride uptake in the adjacent facet joints and intervertebral disc space and found positive areas of uptake in 8/8 (100\%) of lumbar fusion patients. Fischer et al. [7] studied a group of 15 patients with persistent pain after lumbar fusion between one and ten years postoperatively. They observed increased tracer uptake around 8/17 of the cages in the intervertebral disc space, and interpreted values that were higher than normal appearing bone as pathologic. Fischer et al. concluded that ${ }^{18} \mathrm{~F}$-fluoride uptake in the cages was frequently increased, possibly indicating unsuccessful fusion due to increased stresses or micro instability. Quon et al. [11] evaluated the PET/CT results of 22 patients who suffered from recurrent symptoms 4-96 months after spinal fusion surgery, with negative clinical evaluation, physical examination and CT. 15 of the 22 patients underwent revision surgery on 
the basis of abnormal ${ }^{18} \mathrm{~F}$-fluoride uptake at the level of cages, grafts, screws and rods. $\mathrm{PET} / \mathrm{CT}$ correctly predicted the presence of an abnormality observed during revision surgery (cage failure, screw loosening, graft fracture) in 14 of these 15 patients. Seifen et al. [12], evaluated 334 screws in 59 patients on PET/CT to assess screw loosening by calculating the SUV $\max$ surrounding each screw. The final diagnosis was established by surgical exploration in 27 patients and clinical follow-up after in 32 patients. 58 of the 334 screws were positive for loosening with an average SUV $\max$ of $17.10 \pm 6.6 .276$ of the 334 screws were negative for loosening with an average SUV $\max$ of $14.10 \pm 5.9$, which was significantly lower than the SUV $\max$ of the loosened screws. The sensitivity and specificity of ${ }^{18} \mathrm{~F}$-fluoride $\mathrm{PET} / \mathrm{CT}$ were $75 \%, 97.4 \%$ in patient-based analysis, and $45.6 \%, 100 \%$ in screw-based analysis, respectively. Seifen et al. concluded that ${ }^{18} \mathrm{~F}$-fluoride PET/CT provides a high accuracy for identifying implant instability due to screw loosening [12]. Pouldar et al. [10] showed that PET/CT was helpful in identifying pain generators in $17 / 25$ patients with persistent or recurrent back pain over a 15-month period after spinal fusion surgery, verified with either surgical revision and/or facet block or non-invasive treatment. PET/CT findings changed clinical diagnosis and treatment plans. Pouldar et al. stated that PET/CT was consistently able to detect more pathology than $\mathrm{CT}$ alone in postoperative patients, specifically in the evaluation of loosening, degree of fusion and stability and concluded that $\mathrm{PET} / \mathrm{CT}$ is more useful in identifying possible pain sources after spinal fusion than CT alone.

A strength of our study compared to the abovementioned studies was that we studied symptomatic as well as asymptomatic patients in the same study cohort in which we provided a comprehensive evaluation of possible pain generating spinal structures on combined ${ }^{18} \mathrm{~F}$-fluoride PET/CT. By quantifying the amount of pain and disability by means of PROMs, we tried to design an objective analysis method. Furthermore, we attempted to perform analysis methods on CT and on PET/CT that were objectively quantifiable, reproducible and less biased by the individual expertise of a nuclear medicine physician or radiologist. A limitation of our study was that we did not use the obtained PET/CT data for clinical decision making; no intervention or revision surgery was performed based on PET/CT information. In a follow-up study, we would like to base treatment after spinal fusion on PET/CT findings. For example, hotspots at facet joints could indicate a facet joint block and severe hotspots at the endplates could be an indication for revision surgery. Subsequently, we would like to show improved clinical follow-up results or even confirmation of PET/CT findings by observation of abnormalities during revision surgery. Another limitation was the number of patients evaluated in this study. While the total number of patients evaluated was reasonable, the division into subgroups resulted in small remaining numbers in certain categories. For example, we encountered only two patients with screw loosening, which made it impossible to compare our results to the results of Seifen et al. who detected loosening in 58 of the 334 screws [12]. A larger study cohort might provide more significant differences. Also, the follow-up period between 
surgery and PET/CT evaluation was variable in length between patients. Different stages of bone repair mechanisms could have increased variability of the bone metabolism values and biased the results.

At present, ${ }^{18} \mathrm{~F}$-fluoride PET/CT is not yet available in all hospitals. Less advanced nuclear techniques such as a technetium bone scan including SPECT/CT, are widely available and may be used as surrogate parameters [29]. Given the closer correlation with symptomatology, a SPECT/CT or PET/CT scan may be advocated as a routine procedure in patients with persisting symptoms after spinal fusion, and may be viewed as proportional to the overall financial costs and physical and psychological burden for these patients.

To conclude, findings on PET/CT correlated better with PROMs compared to findings on $\mathrm{CT}$. Future studies should demonstrate whether treatments based on PET/CT findings would lead to more benefit for the patient. When interpreting ${ }^{18} \mathrm{~F}$-fluoride PET/CT scans of symptomatic patients after spinal fusion, it is important to realize that bone metabolism patterns of the spinal structures in and around the operated segment might change inherent to the surgical procedure performed. Therefore, not every hotspot on ${ }^{18} \mathrm{~F}$-fluoride $\mathrm{PET} / \mathrm{CT}$ should be directly classified as pathological. 


\section{REFERENCES}

1. Taylor RS, Taylor RJ. The economic impact of failed back surgery syndrome. British journal of pain. 2012; 6(4):174-181.

2. Durand G, Girodon J, Debiais F. Medical management of failed back surgery syndrome in Europe: evaluation modalities and treatment proposals. Neuro-Chirurgie. 2015; 61 Suppl 1:S57-65.

3. Raizman NM, O'Brien JR, Poehling-Monaghan KL, Yu WD. Pseudarthrosis of the spine. The Journal of the American Academy of Orthopaedic Surgeons. 2009; 17(8):494-503.

4. Heggeness MH, Esses SI. Classification of pseudarthroses of the lumbar spine. Spine. 1991; 16(8 Suppl):S449-454.

5. Rothman RH, Booth R. Failures of spinal fusion. The Orthopedic clinics of North America. 1975; 6(1):299304.

6. Brans B, Weijers R, Halders S, Wierts R, Peters M, Punt I, et al. Assessment of bone graft incorporation by $18 \mathrm{~F}$-fluoride positron-emission tomography/computed tomography in patients with persisting symptoms after posterior lumbar interbody fusion. EJNMMI research. 2012; 2(1):42.

7. Fischer DR, Zweifel K, Treyer V, Hesselmann R, Johayem A, Stumpe KD, et al. Assessment of successful incorporation of cages after cervical or lumbar intercorporal fusion with [(18)F]fluoride positron-emission tomography/computed tomography. European spine journal : official publication of the European Spine Society, the European Spinal Deformity Society, and the European Section of the Cervical Spine Research Society. 2011; 20(4):640-648.

8. Gamie S, El-Maghraby T. The role of PET/CT in evaluation of Facet and Disc abnormalities in patients with low back pain using (18)F-Fluoride. Nuclear medicine review Central \& Eastern Europe. 2008; 11(1):1721.

9. Peters $M$, Willems $P$, Weijers $R$, Wierts $R$, Jutten $L$, Urbach $C$, et al. Pseudarthrosis after lumbar spinal fusion: the role of (1)(8)F-fluoride PET/CT. European journal of nuclear medicine and molecular imaging. 2015; 42(12):1891-1898.

10. Pouldar D, Bakshian S, Matthews R, Rao V, Manzano M, Dardashti S. Utility of $18 \mathrm{~F}$ sodium fluoride PET/CT imaging in the evaluation of postoperative pain following surgical spine fusion. Musculoskeletal surgery. 2017.

11. Quon A, Dodd R, lagaru A, de Abreu MR, Hennemann S, Alves Neto JM, et al. Initial investigation of (1)(8)F$\mathrm{NaF}$ PET/CT for identification of vertebral sites amenable to surgical revision after spinal fusion surgery. European journal of nuclear medicine and molecular imaging. 2012; 39(11):1737-1744.

12. Seifen $T$, Rodrigues $M$, Rettenbacher L, Piotrowski W, Holzmannhofer J, Mc Coy M, et al. The value of (18)F-fluoride PET/CT in the assessment of screw loosening in patients after intervertebral fusion stabilization. European journal of nuclear medicine and molecular imaging. 2015; 42(2):272-277.

13. Segall G, Delbeke D, Stabin MG, Even-Sapir E, Fair J, Sajdak R, et al. SNM practice guideline for sodium 18Ffluoride PET/CT bone scans 1.0. Journal of nuclear medicine : official publication, Society of Nuclear Medicine. 2010; 51(11):1813-1820.

14. Lim R, Fahey FH, Drubach LA, Connolly LP, Treves ST. Early experience with fluorine-18 sodium fluoride bone PET in young patients with back pain. Journal of pediatric orthopedics. 2007; 27(3):277-282.

15. Ovadia D, Metser U, Lievshitz G, Yaniv M, Wientroub S, Even-Sapir E. Back pain in adolescents: assessment with integrated 18F-fluoride positron-emission tomography-computed tomography. Journal of pediatric orthopedics. 2007; 27(1):90-93. 
16. Strobel K, Fischer DR, Tamborrini G, Kyburz D, Stumpe KD, Hesselmann RG, et al. 18F-fluoride PET/CT for detection of sacroiliitis in ankylosing spondylitis. European journal of nuclear medicine and molecular imaging. 2010; 37(9):1760-1765.

17. Fischer DR, Maquieira GJ, Espinosa N, Zanetti M, Hesselmann R, Johayem A, et al. Therapeutic impact of [(18)F]fluoride positron-emission tomography/computed tomography on patients with unclear foot pain. Skeletal radiology. 2010; 39(10):987-997.

18. Kobayashi N, Inaba Y, Tezuka T, Ike H, Kubota S, Kawamura M, et al. Evaluation of local bone turnover in painful hip by $18 \mathrm{~F}$-fluoride positron emission tomography. Nuclear medicine communications. 2016; 37(4):399-405.

19. Kobayashi N, Inaba Y, Yukizawa Y, Ike H, Kubota S, Inoue T, et al. Use of 18F-fluoride positron emission tomography as a predictor of the hip osteoarthritis progression. Modern rheumatology. 2015; 25(6):925930.

20. Rauscher I, Beer AJ, Schaeffeler C, Souvatzoglou M, Cronlein M, Kirchhoff C, et al. Evaluation of 18Ffluoride $\mathrm{PET} / \mathrm{MR}$ and $\mathrm{PET} / \mathrm{CT}$ in patients with foot pain of unclear cause. Journal of nuclear medicine : official publication, Society of Nuclear Medicine. 2015; 56(3):430-435.

21. Brenner W, Vernon C, Conrad EU, Eary JF. Assessment of the metabolic activity of bone grafts with (18)Ffluoride PET. European journal of nuclear medicine and molecular imaging. 2004; 31(9):1291-1298.

22. Rigoard P, Blond S, David R, Mertens P. Pathophysiological characterisation of back pain generators in failed back surgery syndrome (part B). Neuro-Chirurgie. 2015; 61 Suppl 1:S35-44.

23. Peters MJ, Wierts R, Jutten EM, Halders SG, Willems PC, Brans B. Evaluation of a short dynamic 18Ffluoride PET/CT scanning method to assess bone metabolic activity in spinal orthopedics. Annals of nuclear medicine. 2015; 29(9):799-809.

24. Fairbank JC, Couper J, Davies JB, O'Brien JP. The Oswestry low back pain disability questionnaire. Physiotherapy. 1980; 66(8):271-273.

25. Ghogawala Z, Resnick DK, Watters WC, 3rd, Mummaneni PV, Dailey AT, Choudhri TF, et al. Guideline update for the performance of fusion procedures for degenerative disease of the lumbar spine. Part 2: assessment of functional outcome following lumbar fusion. Journal of neurosurgery Spine. 2014; 21(1):713.

26. Zanoli G, Stromqvist B, Jonsson B. Visual analog scales for interpretation of back and leg pain intensity in patients operated for degenerative lumbar spine disorders. Spine. 2001; 26(21):2375-2380.

27. Lamers LM, Stalmeier PF, McDonnell J, Krabbe PF, van Busschbach JJ. [Measuring the quality of life in economic evaluations: the Dutch EQ-5D tariff]. Nederlands tijdschrift voor geneeskunde. 2005; 149(28):1574-1578.

28. Lamers LM, McDonnell J, Stalmeier PF, Krabbe PF, Busschbach JJ. The Dutch tariff: results and arguments for an effective design for national EQ-5D valuation studies. Health economics. 2006; 15(10):1121-1132.

29. Hudyana H, Maes A, Vandenberghe T, Fidlers L, Sathekge M, Nicolai D, et al. Accuracy of bone SPECT/CT for identifying hardware loosening in patients who underwent lumbar fusion with pedicle screws. European journal of nuclear medicine and molecular imaging. 2016; 43(2):349-354. 


\section{CHAPTER 5}

\section{Evaluation of a short dynamic ${ }^{18} \mathrm{~F}$-fluoride PET/CT scanning method to assess bone metabolic activity in spinal orthopedics}

Marloes JM Peters§, Roel Wierts $\S$, Elisabeth MC Jutten, Servé GEA Halders, Paul C Willems, Boudewijn T Brans 


\section{ABSTRACT}

\section{OBJECTIVE}

A complication after spinal fusion surgery is pseudarthrosis, but its radiological diagnosis is of limited value. ${ }^{18} \mathrm{~F}$-fluoride PET with its ability to assess bone metabolism activity, could be of value. The goal of this study was to assess the clinical feasibility of calculating the static standardized uptake value (SUV) from a short dynamic scan without the use of blood sampling, thereby obtaining all dynamic and static parameters in a scan of only 30 minutes. This approach was tested on a retrospective patient population with persisting pain after spinal fusion surgery.

\section{METHODS}

In sixteen patients, SUVs (SUVmax, SUV mean) and kinetic parameters ( $K_{1}, k_{2}, k_{3}, V_{b}, K_{i}, N L R$, $\mathrm{K}_{1} / \mathrm{k}_{2}, \mathrm{k}_{3} /\left(\mathrm{k}_{2}+\mathrm{k}_{3}\right), \mathrm{K}_{\mathrm{i}}$, patlak) were derived from static and dynamic PET/CT scans of operated and control regions of the spine, after intravenous administration of $156-214 \mathrm{MBq}{ }^{18} \mathrm{~F}$ fluoride. Parameter differences between control and operated regions, as well as between pseudarthrosis and fused segments were evaluated. SUV mean at 30 and 60 minutes was calculated from kinetic parameters obtained from the dynamic data set (SU-

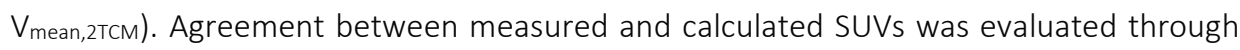
Bland Altman plots.

\section{RESULTS}

Overall, statistically significant differences between control and operated regions were observed for SUV max, SUV mean, $K_{i}, N L R, K_{i}$, patlak, $K_{1} / k_{2}$ and $k_{3} /\left(k_{2}+k_{3}\right)$. Diagnostic CT showed pseudarthrosis in $6 / 16$ patients, while in $10 / 16$ patients, segments were fused. Of all parameters, only those regarding the incorporation of bone ( $\left.K_{\text {bone, }} K_{\text {patlak, }} k_{3} /\left(k_{2}+k_{3}\right)\right)$ differed statistically significant in the intervertebral disc space between the pseudarthrosis and fused patients group. The mean values of the patient-specific blood clearance rate $\tau^{*}$ differed statistically significant between the pseudarthrosis and the fusion group, with a p-value of 0.011. This may correspond with the lack of statistical significance of the SUV values between pseudarthrosis and fused patients. Bland Altman plots show that calculated SUV mean,2TCM values corresponded well with the measured SUV mean values.

\section{CONCLUSION}

This study shows the feasibility of a 30 minute dynamic ${ }^{18} \mathrm{~F}$-fluoride PET/CT scanning and this may provide dynamic parameters clinically relevant to the diagnosis of pseudarthrosis. 


\section{INTRODUCTION}

Low back pain is a major global health and economic problem [1-3], with a 1-year prevalence ranging from 22 to $65 \%$ and life-time prevalence of up to $84 \%$ [4]. The direct costs, including patient care, medical procedures and medication are acceptable, however the yearly indirect costs caused by absence from work and early retirement are manifold [2].

Low back pain is mainly caused by degenerative disorders of the spine, such as spondylolisthesis, degenerative scoliosis, degenerative disc disease, or recurrent disc herniations $[5,6]$. If conservative measures, such as intensive exercise therapy, pain medication or brace immobilization fail, spinal fusion is considered. In at least $15 \%$ of primary lumbar fusions, pseudarthrosis occurs instead of bony fusion [7, 8]. Pseudarthrosis is defined as the absence of solid fusion (nonunion) one year after the operation, and is typically associated with axial or radicular pain [7, 9]. Although solid fusion is not required for pain relief [10], pseudarthrosis in general, even without clinical symptoms, increases the risk of clinical failure, late deformity, neurological symptoms and pain [11].

Surgical exploration remains the gold standard for diagnosing pseudarthrosis [7, 9, 12 15]. Current non-invasive, imaging assessment of pseudarthrosis in patients with persistent or recurrent symptoms after spinal fusion includes plain radiography, flexion-extension radiography, ultrasound, bone scintigraphy, computed tomography (CT) and magnetic resonance imaging (MRI). Most radiological modalities aim at the detection of wellestablished pseudarthrosis by looking at anatomical signs of bony connection between the vertebrae. In contrast, single photon emission computed tomography (SPECT) and positron emission tomography (PET) are three dimensional functional imaging modalities looking at biological processes underlying the process of fusion. Therefore, these imaging techniques may detect an evolving pseudarthrosis. A few studies have reported on the use of PET/CT-scanning for the detection of union/pseudarthrosis after spinal fusion and have indicated the value of ${ }^{18} \mathrm{~F}$-fluoride PET/CT scanning in symptomatic patients [16-19].

These studies have analyzed the PET-images by calculating the standardized uptake value (SUV) to assess bone metabolism. SUV is a valuable tool in clinical practice, easy and fast to use, and can provide reproducible results. However, SUV is dependent on the time after injection [20], and the rate of clearance of the radiotracer from the arterial blood [21]. For ${ }^{18} \mathrm{~F}$-fluoride, Blake et al. has shown that SUV is not optimal in patients with disorders or drugs having an effect on the whole skeleton bone metabolism due to increased blood clearance [22]. Full pharmacokinetic analysis, yielding the fluoride bone influx rate $K_{i}\left(K_{i, N L R}\right.$ and $\left.K_{i, p a t l a k}\right)$ based on the Hawkins model [23], is not dependent on time after injection nor on blood clearance rate. So far, the benefits that dynamic scanning yields, have been outweighed by the practical use and ease of static scanning. Siddique et al. has stated that it is possible to calculate the dynamic parameter $\mathrm{K}_{\mathrm{i}}$ from a static scan in combination with several venous blood samples, obviating the need to make dynamic scans 
[21]. However, this excludes calculation of the individual dynamic parameters that $K_{i}$ is composed of, while the significance of these additional parameters has not been fully explored for this patient population.

The goal of this study was to assess the clinical feasibility of obtaining dynamic and static parameters from a 30 minutes scan without the use of blood sampling, and compare these parameters in a retrospective patient population with persisting pain after spinal fusion surgery.

\section{MATERIALS AND METHODS}

\section{Patients}

A cohort of sixteen patients was enrolled in this study between June 2008 and February 2015. Patients who underwent posterior lumbar interbody fusion (PLIF) surgery for the indication 1-2 grade degenerative spondylolisthesis, and who suffered from persisting or recurrent low back pain after the procedure without an obvious clinical or radiological explanation were included in the study. The patient group consisted of 11 female and 5 male patients, with a mean age at surgery of 44.9 years (range: $26-64$ years) and a body mass index (BMI) of $29.5 \mathrm{~kg} / \mathrm{m}^{2}$ (range: $19.3-44.6 \mathrm{~kg} / \mathrm{m}^{2}$ ). Patients were operated on level L3-L4 ( $n=2)$, L4-L5 ( $n=4)$ or L5-S1 ( $=10)$. The time interval between fusion surgery and the PET/CT examination was 4 to 75 months (mean 22 months, median 17 months). One patient underwent PLIF surgery at two levels. Therefore, the total number of operated levels to be analyzed was 17 . This study was performed in accordance with the Helsinki Declaration of 1975, as revised in 2013, and was part of a protocol accepted by the medical ethical committee of the Maastricht University Medical Center (NL.32881.068.11) in which patients gave their written and informed consent.

Posterior Lumbar Interbody Fusion (PLIF), surgical technique

Under general anesthesia and in a prone position, the vertebral arches of the intended levels were identified under fluoroscopic control and exposed by an open posterior lumbar approach. Nerve roots were decompressed by laminectomy and the intervertebral disc was excised. After thorough cleansing of the endplates, two 10-12 mm intervertebral cages (Capstone ${ }^{\circledR}$ PEEK, Medtronic, Memphis, U.S.A.), filled with autologous bone from the vertebral lamina, were inserted into the disc space, right and left of the midline. Additionally, the remaining disc space was packed with autologous bone chips from the laminectomy. Next, the upper and lower vertebrae were fixed by four transpedicular screws with titanium rods (CD Legacy ${ }^{\bullet}$, Medtronic, Memphis, U.S.A.) for primary stabilization. 


\section{${ }^{18}$ F-fluoride PET/CT-scans}

The PET and CT-images were acquired with an integrated PET/CT-scanner (Gemini TF PETCT, Philips, The Netherlands). First, a low-dose CT acquisition (120 kV, 30 mAs, slice thickness $4 \mathrm{~mm}$ ) used for localization purposes and attenuation correction of the PET-images was made. Immediately after intravenous injection of 156-214 MBq (mean $188 \mathrm{MBq}$; median $186 \mathrm{MBq}) \mathrm{Na}-\left({ }^{18} \mathrm{~F}\right)$-fluoride, the dynamic scanning started which involved a 3-dimensional 30 minute list mode PET-scan of the operated segment in an $18 \mathrm{~cm}$ axial field-ofview. This list mode scan was rebinned into the following consecutive time frames: $6 \times 5$ s, $3 \times 10$ s, $9 \times 60$ s, $10 \times 120$ s. Static scanning involved a low-dose CT acquisition (parameters as in the dynamic case) followed by a conventional PET-scan 60 minutes after injection, covering the whole lumbosacral spine, acquired by two bed positions of five minutes each. This was immediately followed by a high-dose, non-contrast enhanced CT scan (64slice helical, $120 \mathrm{kV}, 250 \mathrm{mAs}$, slice $1 \mathrm{~mm}$ with increment of $0.8 \mathrm{~mm}$ ) of the fusion region. Standard filtered backprojection CT-reconstruction was performed. PET-images were reconstructed into both non-attenuated and CT-based attenuated images using the standard blob-os-TF reconstruction algorithm. Images were viewed on clinical software (EBW, Philips, The Netherlands) and further analyzed by a research tool (PMOD 3.0, PMOD Technologies Ltd, Zürich).

\section{Analysis of ${ }^{18}$ F-fluoride PET/CT-scans}

Twelve parameters were derived from the static and dynamic PET scans. The mean and maximum SUV at 30 minutes were calculated from the last frame of the dynamic scan $\left(S U V_{\text {mean30 }}\right.$ and $\left.S U V_{\max 30}\right)$. The mean and maximum SUV at 60 minutes were calculated from the static scan $\left(S U V_{\text {mean6o }}\right.$ and $\left.S U V_{\operatorname{max60}}\right)$. The analysis of the dynamic scans was based on the 2 tissue compartment model (2TCM) [23]. Through nonlinear regression (NLR) analysis, $K_{1}, k_{2}, k_{3}, v_{b}, K_{i}, N L R, K_{1} / k_{2}$ and $k_{3} /\left(k_{2}+k_{3}\right)$ were calculated from the dynamic scan. $K_{i}$, patlak was calculated from the dynamic scan through Patlak graphical analysis [24, 25].

The twelve parameters were calculated based on a region of interest (ROI) approach. In each CT-scan six ellipsoid shaped ROls were manually drawn following the contours of the vertebrae (slice thickness $4 \mathrm{~mm}$, short axis range: $40-50 \mathrm{~mm}$, long axis range: 55-65 $\mathrm{mm}$ ), including the intervertebral disc space and upper and lower endplates of the operated segment (referred to as inter, endUP, endLOW) as well as of a control segment two levels higher (referred to as inter_C, endUP_C, endLOW_C) (Figure 1A). These ROIs were transferred to the co-registered attenuation corrected PET image (Figure 1B). 


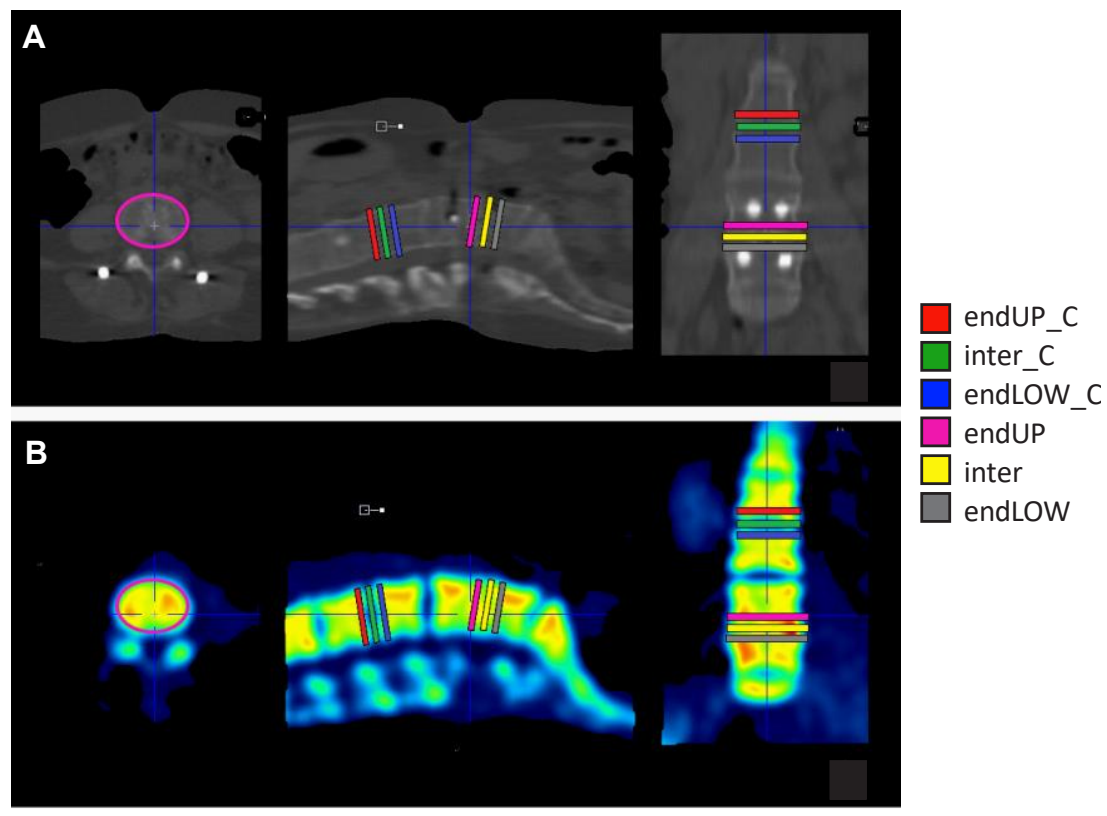

Figure 1. (A) An axial, sagittal and coronal CT-image (from left to right) of the lower spine after PLIF. Three ROIs were drawn in the operated segment: the lower endplate of the cranial vertebra (endUP in pink), the intervertebral disc (inter in yellow), the upper endplate of the caudal vertebra (endLOW in grey). The same three ROIs were drawn in a normal reference segment two levels above the operated segment (endUP_C in red, inter_C in green and endLOW_C in blue, respectively). (B) The six ROIs were transferred to the co-registered PET-image (axial, sagittal and coronal views from left to right).

1. SUV was obtained by correcting the radioactivity concentration measured by the PETscanner $(\mathrm{A}[\mathrm{kBq} / \mathrm{ml}])$ for the injected dose of ${ }^{18} \mathrm{~F}$-fluoride (ID [MBq]) and the body weight of the patient $(\mathrm{m}[\mathrm{kg}])$ according to (1).

$$
S U V=\frac{A}{I D / m}
$$

2. For dynamic analysis, the arterial blood input function and the tissue time-activity curves (TACS) were needed. The same six ROls that were used in static analysis were applied to the dynamic frames to generate the TACs. The arterial blood input function was determined by means of an image derived input function (IDIF) obtained via a thresholding method. The frames of the dynamic PET scan that showed a clear bolus (2-4 frames early in the dynamic scan) were summed. In the summed image, a $75 \%$ threshold was applied to a box placed manually around the abdominal aorta, to obtain a volume of interest (VOI). By applying the VOI to all dynamic frames, the IDIF was generated.

The IDIF and ROI TACs were fitted to the 2TCM using a nonlinear regression algorithm and a Patlak algorithm (PMOD 3.0, PMOD Technologies Ltd, Zürich) estimating the kinetic parameters $K_{1}, k_{2}, k_{3}, v_{b}$ and $K_{i}$, patlak. The parameter $k_{4}$ was assumed to be negligible and set to 0 . 
The fluoride bone influx rate, $\mathrm{K}_{\mathrm{i}}$ (previously referred to in literature as $\mathrm{K}_{\text {bone), }}$, represents the net uptake rate of ${ }^{18} \mathrm{~F}$-fluoride in the bone mineral, calculated as a combination of the rate constants (2). $K_{1}$ is correlated to bone perfusion [26]. Also calculated was $k_{3} /\left(k_{2}+k_{3}\right)$, which represents the fraction of tracer entering the tissue compartment that undergoes specific binding to the bone mineral [26] and $K_{1} / k_{2}$, which represents the volume of distribution of tracer in the unbound pool [27].

$$
K_{i, N L R}=\frac{K_{1} \cdot k_{3}}{k_{2}+k_{3}}
$$

3. The relationship between SUV and $K_{i}$, NLR can be written as (3), which was derived using the analytical solution of the 2TCM for the time-dependent tissue radioactivity concentration. In which $\mathrm{f}_{\mathrm{b}}$ represents the blood fraction $[\mathrm{L} / \mathrm{kg}$ ] defined as the ratio of total blood volume to body mass and $\tau[\mathrm{Bq} \cdot \mathrm{s} / \mathrm{Bq}]$ is the blood fractional residence time in a region, defined as the mean time that an administered substance spends in that region [28]. The full derivation can be found in the Appendix.

$$
\lim _{t \rightarrow \infty} S U V_{2 T C M} \approx K_{i, N L R} \cdot \frac{\tau}{f_{\mathrm{b}}}=K_{i, N L R} \cdot \tau^{*}
$$

In (3), the factor $\tau^{*}[\mathrm{~Bq} \cdot \mathrm{s} / \mathrm{Bq}]$ is defined as the blood residence time normalized to the blood fraction. The residence time as well as the blood fraction are patient specific factors that are not directly related to metabolic bone activity at a specific site of interest but do have an effect on SUV. This relationship together with the $K_{i, N L R}$ values were used to calculate SUV at 30 and at 60 minutes from the 30 minute dynamic scan (SUV mean30,2TCM and SUV mean60,2TCM respectively). Moreover, the factor $\tau^{*}$ was calculated for each patient to evaluate the inter-subject variability that this factor introduces to the SUV.

Based on the CT scan, patients were divided into two categories. Patients who had no signs of bony bridging between the two operated vertebrae were categorized as pseudarthrosis. Patients with bony bridges were categorized as fused.

\section{Statistical analysis}

Statistical evaluation was performed using IBM SPSS Statistics for Windows, Version 20.0 (Armonk, NY: IBM Corporation). To test the data for normality of distribution, the ShapiroWilk test was used. The Pearson correlation test was used to examine the correlation between the different methods. The goodness of the fit was determined through calculation of $\mathrm{R}^{2}$. The differences between the control and the operated regions, and between the pseudarthrosis and fused patients were compared using the Wilcoxon signed rank test. The magnitude of the observed differences between operated and control regions was evaluated by calculation of the Cohen's d effect size, which is defined as the difference between the two means of the subgroups divided by the standard deviation of the complete data set. In practice, the higher (positive or negative) value of Cohen's d effect 
size, the larger the difference. To determine whether the calculated SUV agreed with the measured SUV, Bland Altman plots were evaluated [29]. P-values smaller than or equal to 0.05 were considered statistically significant.

\section{RESULTS}

Table 1 summarizes the fluoride bone metabolic values, i.e. SUV, Patlak and NLR rate constants for the control and the operated regions. As can be seen, statistically significant differences between control and operated regions (upper, lower endplate and intervertebral) were found for most of the obtained parameters, although not for the individual rate constants $K_{1}, k_{2}, k_{3}$ and $v_{b}$. The highest statistical significance values were found for SUVmean60 ( $p$-value range: 0.001-0.002), $K_{i}$, patlak ( $p$-value range: 0.001-0.003), $K_{i}, N L R, K_{1} / K_{2}$ ( $p$-value range: 0.004-0.03) and $k_{3} /\left(k_{2}+k_{3}\right)$ ( $p$-value range: 0.003-0.02).

When comparing the Cohen's d effect size of the operated to the control regions, parameters concerning bone metabolism, (SUV mean60, SUV $\operatorname{max60,} \mathrm{K}_{\mathrm{i} \text {,patlak }}$ and $\mathrm{K}_{\mathrm{i}, \mathrm{NLR}}$ ) showed larger differences for the intervertebral disc space (around 3.5) than for the endplates (around 1.5). This corresponded to the increase of bone turnover in the fusion region in contrast to the non-osseous normal intervertebral disc space. Equation (2) shows that bone me-

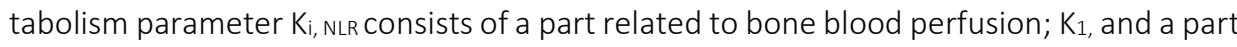
related to bone incorporation; $k_{3} /\left(k_{2}+k_{3}\right)$. As can be seen in Table 1 , looking at the intervertebral values, the effect size for $k_{3} /\left(k_{2}+k_{3}\right)$ was 2.5 whereas the effect size for $k_{1}$ was only 0.70 , indicating that the high effect size of $K_{i}, N L R$, was mainly caused by a higher amount of bone incorporation and not due to an increase in $\mathrm{K}_{1}$.

Figure 2 and figure 3 show the relationship between $K_{i, N L R}$ and $K_{i}$, patlak and between SUV and $K_{i}$ values respectively. Figure $2 A$ shows a very high correlation between $K_{i}$, NLR and $K_{i}$, patlak (Pearson correlation value of $0.98, p<0.0001$ ), indicating that the two dynamic approaches are robust. Figure $2 \mathrm{~B}$ shows a Bland Altman plot for the data shown in Figure 2A. The mean difference between $K_{i, N L R}$ and $K_{i, p a t l a k}$ was 0.0016 with $95 \%$ limits of agreement of -0.0077 and +0.011 . Moreover, the trend line almost coincided with the line of identity, indicating that $K_{i}, N L R$ and $K_{i}$, patlak yield identical results. Figure 3 shows the correlation of the measured SUV mean30 (Figure 3A) and SUV mean60 (Figure 3B) with $K_{i, \text { NLR. Both }}$ SUV $V_{\text {mean30 }}$ and SUV $V_{\text {mean6o }}$ were highly correlated to $K_{i}$, NLR $\left(R^{2}=0.82\right.$ and $R^{2}=0.64$ respectively). Despite this good correlation, it can be seen from the figures that for individual regions, rather large deviations from the trend line were present. 
Table 1. Parameter values of control and operated regions. P-values $\leq 0.05$ are presented in red font.

\begin{tabular}{|c|c|c|c|c|c|}
\hline Parameter & $\begin{array}{l}\text { Region of } \\
\text { interest }^{a}\end{array}$ & $\begin{array}{c}\text { Control } \\
\text { regions } \\
{[\text { mean } \pm \mathrm{SD}]^{b}}\end{array}$ & $\begin{array}{c}\text { Operated } \\
\text { regions } \\
{[\text { mean } \pm \mathrm{SD}]^{\mathrm{b}}}\end{array}$ & $p$-value ${ }^{c}$ & Effect size $^{b}$ \\
\hline \multirow{3}{*}{$\begin{array}{c}\mathrm{SUV}_{\text {mean30 }} \\
{[-]}\end{array}$} & endUP & $5.0 \pm 1.2$ & $7.7 \pm 2.2$ & 0.002 & 1.6 \\
\hline & inter & $2.3 \pm 0.74$ & $7.0 \pm 2.1$ & 0.001 & 3.6 \\
\hline & endLOW & $5.1 \pm 1.1$ & $7.8 \pm 2.0$ & 0.001 & 1.3 \\
\hline \multirow{3}{*}{$\begin{array}{c}\text { SUV }_{\max 30} \\
{[-]}\end{array}$} & endUP & $6.7 \pm 1.7$ & $11 \pm 3.3$ & 0.002 & 1.8 \\
\hline & inter & $3.6 \pm 1.5$ & $11 \pm 3.1$ & 0.001 & 3.3 \\
\hline & endLOW & $7.2 \pm 1.9$ & $11 \pm 3.2$ & 0.001 & 1.6 \\
\hline \multirow{3}{*}{$\begin{array}{c}S U V_{\text {mean60 }} \\
{[-]}\end{array}$} & endUP & $6.2 \pm 1.1$ & $8.6 \pm 2.3$ & 0.002 & 1.6 \\
\hline & inter & $2.6 \pm 0.83$ & $7.3 \pm 2.1$ & 0.001 & 3.3 \\
\hline & endLOW & $6.4 \pm 1.2$ & $8.9 \pm 2.5$ & 0.002 & 1.3 \\
\hline \multirow{3}{*}{$\begin{array}{c}\mathrm{SUV}_{\max 60} \\
{[-]}\end{array}$} & endUP & $8.4 \pm 1.6$ & $13 \pm 3.8$ & 0.001 & 1.5 \\
\hline & inter & $4.4 \pm 2.5$ & $12 \pm 3.2$ & 0.001 & 3.6 \\
\hline & endLOW & $9.1 \pm 2.8$ & $14 \pm 4.9$ & 0.005 & 1.3 \\
\hline \multirow{3}{*}{$\begin{array}{l}\mathrm{K}_{\mathrm{i}, \text { patlak }} \\
{\left[\mathrm{min}^{-1}\right]}\end{array}$} & endUP & $0.053 \pm 0.012$ & $0.081 \pm 0.023$ & 0.003 & 1.7 \\
\hline & inter & $0.020 \pm 0.0072$ & $0.075 \pm 0.022$ & 0.001 & 3.4 \\
\hline & endLOW & $0.050 \pm 0.011$ & $0.084 \pm 0.021$ & 0.001 & 1.9 \\
\hline \multirow{3}{*}{$\begin{array}{l}\mathrm{K}_{\mathrm{i}, \mathrm{NLR}} \\
{\left[\mathrm{min}^{-1}\right]}\end{array}$} & endUP & $0.055 \pm 0.012$ & $0.079 \pm 0.020$ & 0.002 & 1.8 \\
\hline & inter & $0.022 \pm 0.0064$ & $0.073 \pm 0.025$ & 0.001 & 3.5 \\
\hline & endLOW & $0.053 \pm 0.011$ & $0.076 \pm 0.029$ & 0.011 & 1.8 \\
\hline \multirow{3}{*}{$\begin{array}{c}\mathrm{K}_{1} \\
{\left[\mathrm{ml} \cdot \mathrm{g}^{-1} \cdot \mathrm{min}^{-1}\right]}\end{array}$} & endUP & $0.23 \pm 0.12$ & $0.19 \pm 0.027$ & 0.463 & -0.29 \\
\hline & inter & $0.14 \pm 0.065$ & $0.18 \pm 0.037$ & 0.022 & 0.70 \\
\hline & endLOW & $0.20 \pm 0.067$ & $0.20 \pm 0.076$ & 0.594 & -0.065 \\
\hline \multirow{3}{*}{$\begin{array}{c}\mathrm{k}_{2} \\
{\left[\mathrm{~min}^{-1}\right]}\end{array}$} & endUP & $1.0 \pm 1.7$ & $0.23 \pm 0.21$ & 0.019 & -0.63 \\
\hline & inter & $0.41 \pm 0.25$ & $0.27 \pm 0.25$ & 0.140 & -0.61 \\
\hline & endLOW & $0.27 \pm 0.13$ & $0.32 \pm 0.64$ & 0.056 & 0.12 \\
\hline \multirow{3}{*}{$\begin{array}{c}\mathrm{k}_{3} \\
{\left[\mathrm{~min}^{-1}\right]}\end{array}$} & enduP & $0.21 \pm 0.19$ & $0.14 \pm 0.13$ & 0.055 & -0.33 \\
\hline & inter & $0.076 \pm 0.039$ & $0.16 \pm 0.15$ & 0.026 & 0.87 \\
\hline & endLOW & $0.098 \pm 0.041$ & $0.15 \pm 0.15$ & 0.363 & 0.51 \\
\hline \multirow{3}{*}{$\begin{array}{l}V_{b} \\
{[-]}\end{array}$} & endUP & $0.027 \pm 0.016$ & $0.041 \pm 0.017$ & 0.003 & 0.99 \\
\hline & inter & $0.032 \pm 0.011$ & $0.040 \pm 0.017$ & 0.022 & 0.50 \\
\hline & endLOW & $0.041 \pm 0.019$ & $0.040 \pm 0.018$ & 0.975 & -0.23 \\
\hline \multirow{3}{*}{$\begin{array}{c}\mathrm{K}_{1} / \mathrm{K}_{2} \\
{\left[\mathrm{ml} \cdot \mathrm{g}^{-1}\right]}\end{array}$} & endUP & $0.56 \pm 0.33$ & $2.2 \pm 2.4$ & 0.004 & 0.84 \\
\hline & inter & $0.42 \pm 0.21$ & $1.8 \pm 2.0$ & 0.003 & 1.0 \\
\hline & endLOW & $0.89 \pm 0.32$ & $3.2 \pm 5.5$ & 0.030 & 0.47 \\
\hline \multirow{3}{*}{$\begin{array}{c}\mathrm{k}_{3} /\left(\mathrm{k}_{2}+\mathrm{k}_{3}\right) \\
{[-]}\end{array}$} & endUP & $0.29 \pm 0.14$ & $0.41 \pm 0.099$ & 0.003 & 1.3 \\
\hline & inter & $0.18 \pm 0.088$ & $0.40 \pm 0.14$ & 0.004 & 2.5 \\
\hline & endLOW & $0.29 \pm 0.089$ & $0.41 \pm 0.20$ & 0.016 & 1.2 \\
\hline
\end{tabular}

\footnotetext{
${ }^{a}$ Abbreviations: endUP=upper endplate; inter= intervertebral disc space; endLOW=lower endplate

b Values with 2 significant digits

c The $\mathrm{p}$-value indicates the statistical difference between the operated and control region
} 

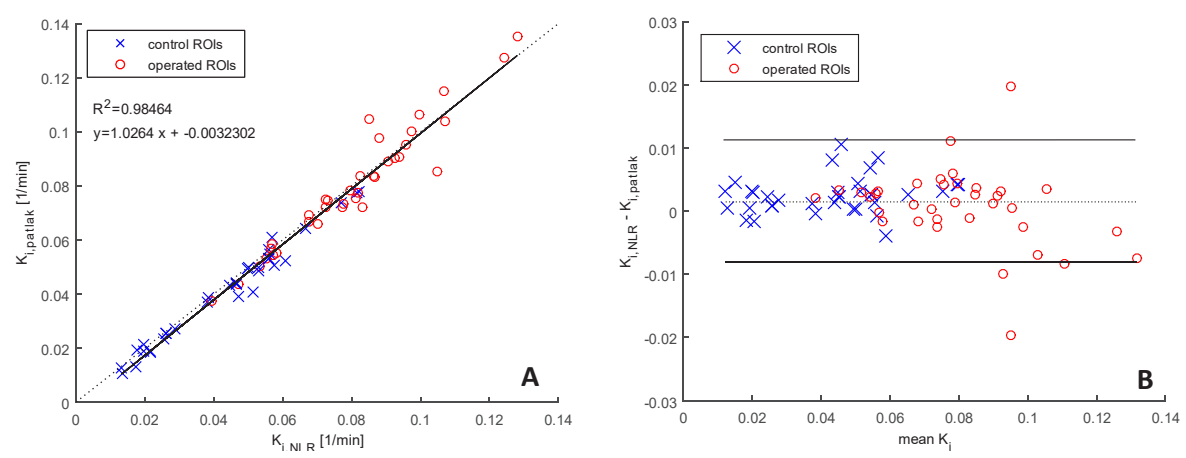

Figure 2. (A) Pearson correlation plot with correlation value $R^{2}(p<0.0001)$ between $K_{i}$, NLR and $K_{i}$, pattak. Blue crosses represent the control region data and red circles represent the operated region data. The dotted line in the graph is the line of identity. Equation $y$ in the figure shows the deviation of the data from the line of identity, representing the difference between the two parameters. (B) Bland Altman plot for the data shown in (A). The dashed horizontal line represents the mean difference value, the solid horizontal lines represent the $95 \%$ confidence interval.
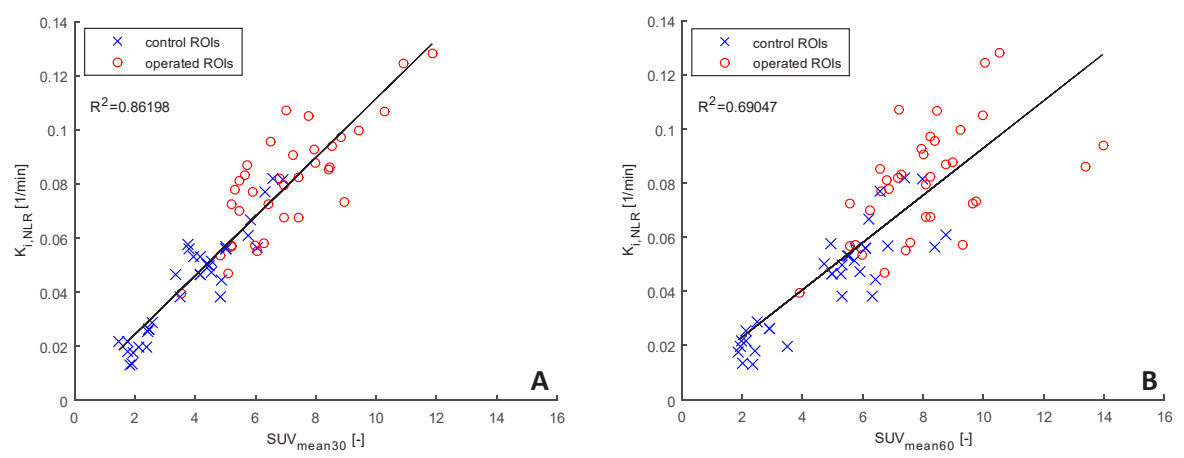

Figure 3. Pearson correlation plot with correlation values $(p<0.0001)$ between $S U V_{\text {mean60 }}(A), S U V_{\text {mean30 }}(B)$ and

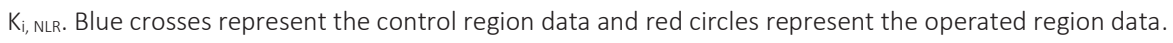

Figure 4 shows the relationship between the measured SUV mean at 30 (Figure 4A; SU-

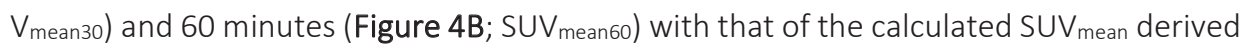
from the dynamic data (SUV mean 30/60,2TCM). It can be observed that the deviation from the trend line decreased compared to Figure 3 , which was supported by an increase in $\mathrm{R}^{2}$ values to 0.93 and 0.78 respectively. With equation (3) in mind, this improvement in correlation after addition of $\tau^{*}$ indicated that the residence time was a patient specific factor that added variability to SUV not directly related to local bone incorporation $\left(K_{i}\right)$. 

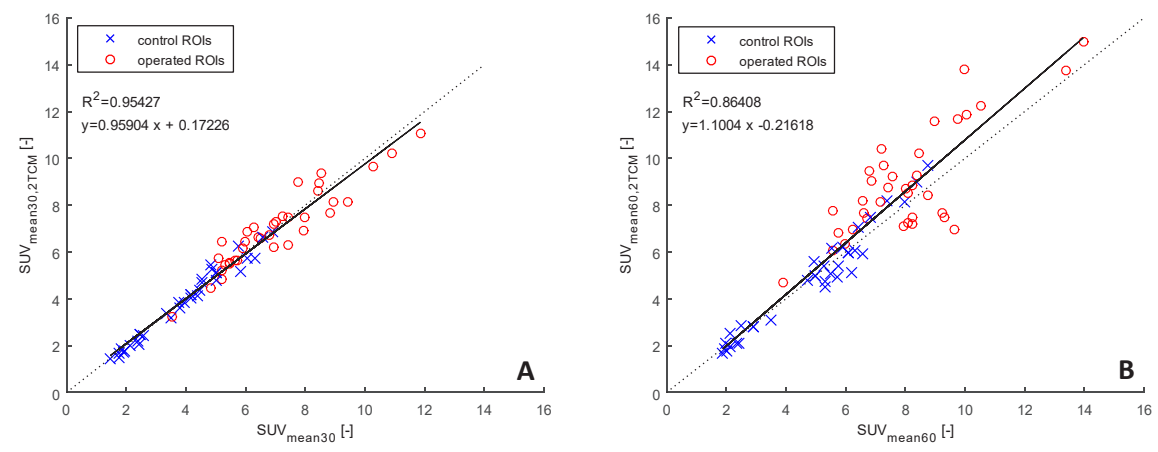

Figure 4. Pearson correlation plots with correlation values between the measured SUV mean3o and the calculated $S U V_{\text {mean30,2TCM }}(A)$, and SUV mean60 and SUV mean60,2TCM (B) $(p<0.0001)$. Blue crosses represent the control region data and red circles represent the operated region data. The dotted line in the graph is the line of identity. Equation y shows the deviation of the data from the line of identity, representing the difference between the two parameters.

Figure 5 shows the Bland Altman plots for the data shown in Figure 4. The mean difference between SUV mean30 and SUV mean302TCM was -0.052 with $95 \%$ limits of agreement of 1.3 and +1.1 . The mean difference between $S U V_{\text {mean60 }}$ and $S U V_{\text {mean602TCM }}$ was +0.43 with larger $95 \%$ limits of agreement of -2.3 and 3.0. In both Bland Altman plots, only a few regions exceed the $95 \%$ confidence interval $(\mathrm{CI})$ limits. Using SUV mean30,2TCM, a measured SUV $V_{\text {mean30 }}$ of 8 would be calculated from the dynamic data to lie between 7 and 9 with a $\mathrm{Cl}$ of 95\%. Using SUV mean60,2TCM, a measured SUV mean60 of 8 would lie between 6 and 10.5 with a $\mathrm{Cl}$ of $95 \%$. Thus, values of the calculated SUVmean60, 2тсм from the dynamic $30 \mathrm{mi}-$ nute scan corresponded well with the measured SUV mean60 from the static scan at 60 minutes post injection.
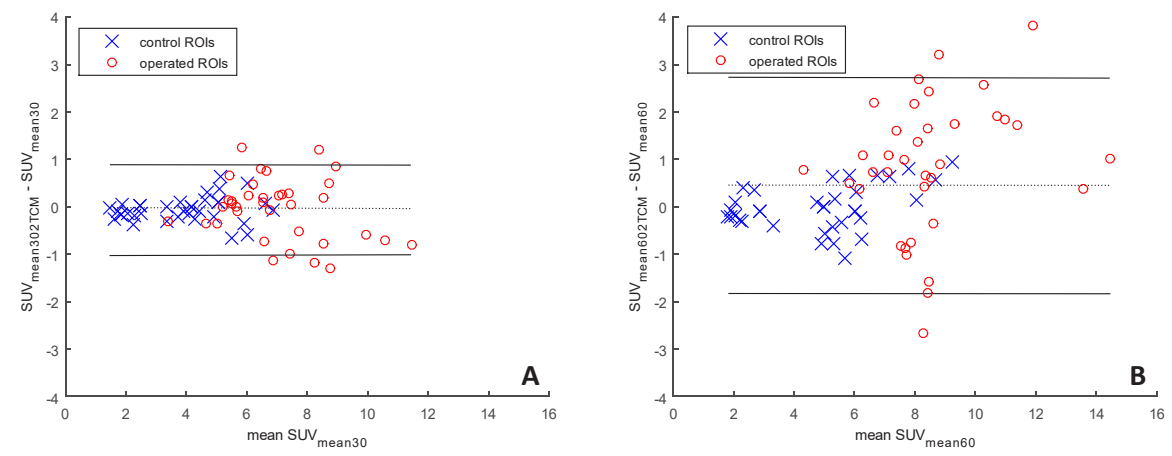

Figure 5. Bland Altman plots, mean SUV measured from the PET scan (SUV mean 30, SUV mean60) (A) and calculated

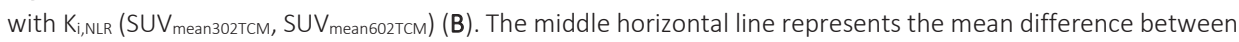
SUV mean and SUV mean2TCM. The upper and lower lines represent the $95 \%$ confidence interval limits. Blue crosses represent the control region data and red circles represent the operated region data. 
Table 2 shows the parameters in the pseudarthrosis $(n=6)$ and fused $(n=10)$ patient group. Of all parameters obtained, only those regarding the incorporation of bone ( $K_{\text {bone, }} K_{\text {patlak, }}$ $\left.k_{3} /\left(k_{2}+k_{3}\right)\right)$ differed statistically significant in the intervertebral disc space between the pseudarthrosis and fused patients group. The values of $\tau^{*}$ range between $64.4 \mathrm{~Bq} \cdot \mathrm{s} / \mathrm{Bq}$ and $115 \mathrm{~Bq} \cdot \mathrm{s} / \mathrm{Bq}$ with a standard deviation of $13.4 \mathrm{~Bq} \cdot \mathrm{s} / \mathrm{Bq}$, which is $16.3 \%$ of the mean value $81.9 \mathrm{~Bq} \cdot \mathrm{s} / \mathrm{Bq}$. For the pseudarthrosis group, $\tau^{*}$ had a mean of 87.4 with a standard deviation of 7.2. For the fusion group, $\tau^{*}$ had a mean of 74.3 with a standard deviation of 7.4. The mean values of $\tau^{*}$ differed statistically significant between the pseudarthrosis and the fusion group, with a p-value of 0.011 . This may correspond with the lack of statistical significance of the SUV values. 
Table 2. Parameter values in operated regions for pseudarthrosis and fusion patients. P-values $\leq 0.05$ are presented in red font.

\begin{tabular}{|c|c|c|c|c|}
\hline Parameter & Region of interest ${ }^{a}$ & $\begin{array}{l}\text { Pseudarthrosis } \\
\qquad(n=6) \\
{\text { [mean } \pm S D]^{b}}^{b}\end{array}$ & $\begin{array}{c}\text { Fused } \\
(n=10) \\
{[\text { mean } \pm S D]^{b}}\end{array}$ & $p$-value ${ }^{c}$ \\
\hline \multirow{3}{*}{$\begin{array}{c}\text { SUV }_{\text {mean } 30} \\
{[-]}\end{array}$} & endUP & $8.5 \pm 2.1$ & $7.2 \pm 2.1$ & 0.279 \\
\hline & inter & $7.3 \pm 2.4$ & $6.7 \pm 1.8$ & 0.786 \\
\hline & endLOW & $8.7 \pm 2.1$ & $7.2 \pm 1.6$ & 0.144 \\
\hline \multirow{3}{*}{$\begin{array}{c}\mathrm{SUV}_{\max 30} \\
{[-]}\end{array}$} & enduP & $13 \pm 3.6$ & $10 \pm 3.0$ & 0.100 \\
\hline & inter & $12 \pm 3.5$ & $11 \pm 3.0$ & 0.524 \\
\hline & endLOW & $13 \pm 3.5$ & $11 \pm 2.8$ & 0.160 \\
\hline \multirow{3}{*}{$\begin{array}{c}\text { SUV } \\
{[-]}\end{array}$} & endUP & $10 \pm 3.2$ & $8.0 \pm 1.4$ & 0.275 \\
\hline & inter & $7.3 \pm 2.7$ & $7.5 \pm 1.3$ & 0.622 \\
\hline & endLOW & $11 \pm 3.3$ & $8.0 \pm 1.2$ & 0.126 \\
\hline \multirow{3}{*}{$\begin{array}{c}\text { SUV }_{\max 60} \\
{[-]}\end{array}$} & enduP & $17 \pm 5.8$ & $12 \pm 2.3$ & 0.145 \\
\hline & inter & $14 \pm 5.1$ & $12 \pm 2.6$ & 0.617 \\
\hline & endLOW & $17 \pm 5.9$ & $12 \pm 3.2$ & 0.170 \\
\hline \multirow{3}{*}{$\begin{array}{l}\mathrm{K}_{\mathrm{i}, \text { patlak }} \\
{\left[\mathrm{min}^{-1}\right]}\end{array}$} & endUP & $0.077 \pm 0.012$ & $0.082 \pm 0.026$ & 0.551 \\
\hline & inter & $0.062 \pm 0.018$ & $0.081 \pm 0.020$ & 0.050 \\
\hline & endLOW & $0.082 \pm 0.017$ & $0.083 \pm 0.022$ & 0.810 \\
\hline \multirow{3}{*}{$\begin{array}{l}\mathrm{K}_{\mathrm{i}, \mathrm{NLR}} \\
{\left[\mathrm{min}^{-1}\right]}\end{array}$} & endUP & $0.07 \pm 0.013$ & $0.081 \pm 0.021$ & 0.355 \\
\hline & inter & $0.045 \pm 0.019$ & $0.083 \pm 0.019$ & 0.004 \\
\hline & endLOW & $0.07 \pm 0.015$ & $0.084 \pm 0.020$ & 0.137 \\
\hline \multirow{3}{*}{$\frac{\mathrm{K}_{1}}{\left[\mathrm{ml} \cdot \mathrm{g}^{-1} \cdot \mathrm{min}^{-1}\right]}$} & endUP & $0.19 \pm 0.023$ & $0.19 \pm 0.036$ & 0.937 \\
\hline & inter & $0.15 \pm 0.038$ & $0.18 \pm 0.045$ & 0.355 \\
\hline & endLOW & $0.18 \pm 0.023$ & $0.20 \pm 0.090$ & 0.516 \\
\hline \multirow{3}{*}{$\begin{array}{c}\mathrm{k}_{2} \\
{\left[\mathrm{~min}^{-1}\right]}\end{array}$} & endUP & $0.19 \pm 0.11$ & $0.25 \pm 0.24$ & 0.333 \\
\hline & inter & $0.22 \pm 0.16$ & $0.29 \pm 0.28$ & 0.454 \\
\hline & endLOW & $0.16 \pm 0.094$ & $0.39 \pm 0.75$ & 0.448 \\
\hline \multirow{3}{*}{$\begin{array}{c}\mathrm{k}_{3} \\
{\left[\mathrm{~min}^{-1}\right]}\end{array}$} & endUP & $0.10 \pm 0.045$ & $0.17 \pm 0.15$ & 0.266 \\
\hline & inter & $0.09 \pm 0.063$ & $0.22 \pm 0.18$ & 0.066 \\
\hline & endLOW & $0.10 \pm 0.055$ & $0.19 \pm 0.18$ & 0.269 \\
\hline \multirow{3}{*}{$\begin{array}{l}\mathrm{Vb} \\
{[-]}\end{array}$} & endUP & $0.047 \pm 0.019$ & $0.037 \pm 0.012$ & 0.152 \\
\hline & inter & $0.046 \pm 0.020$ & $0.037 \pm 0.013$ & 0.547 \\
\hline & endLOW & $0.046 \pm 0.020$ & $0.036 \pm 0.013$ & 0.431 \\
\hline \multirow{3}{*}{$\begin{array}{c}\mathrm{K}_{1} / \mathrm{K}_{2} \\
{\left[\mathrm{ml} \cdot \mathrm{g}^{-1}\right]}\end{array}$} & enduP & $1.8 \pm 1.9$ & $2.1 \pm 2.5$ & 0.894 \\
\hline & inter & $0.6 \pm 2.1$ & $1.6 \pm 1.8$ & 0.879 \\
\hline & endLOW & $1.9 \pm 1.9$ & $2.9 \pm 5.6$ & 0.759 \\
\hline \multirow{3}{*}{$\begin{array}{c}\mathrm{k}_{3} /\left(\mathrm{k}_{2}+\mathrm{k}_{3}\right) \\
{[-]}\end{array}$} & endUP & $0.36 \pm 0.044$ & $0.44 \pm 0.12$ & 0.215 \\
\hline & inter & $0.29 \pm 0.091$ & $0.47 \pm 0.13$ & 0.016 \\
\hline & endLOW & $0.40 \pm 0.067$ & $0.46 \pm 0.18$ & 0.506 \\
\hline
\end{tabular}

\footnotetext{
${ }^{a}$ Abbreviations: endUP=upper endplate; inter= intervertebral disc space; endUP=Iower endplate

${ }^{b}$ Values with 2 significant digits

${ }^{\mathrm{c}}$ The $\mathrm{p}$-value indicates the statistical difference between the pseudarthrosis and fusion patients
} 


\section{DISCUSSION}

Blake has shown that the dependency of SUV on clearance rate can lead to erroneous conclusions as opposed to $K_{i}$ which is independent of clearance rate [22]. Small differences were only detectable with $K_{i}[30,31]$. Brenner found, in a study on limb and thoracic spine bone grafts, that with $\mathrm{K}_{\mathrm{i}}$, smaller changes in bone metabolism could be detected than with SUV due to a wider $95 \%$ range of results for SUV $( \pm 58.0 \%)$ as compared

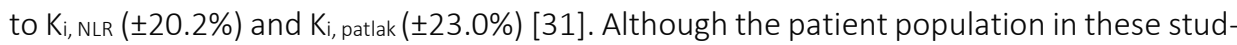
ies might not be representative for our present population, it shows the effect that differences in clearance can have on results. In our study, the rate of clearance was incorporated in the parameter $\tau^{*}$. It was shown that in this particular patient population, the patient specific factor $\tau *$ introduced an additional inter-subject variability of $16.3 \%$ to SUV that was not directly related to local bone metabolism. Table 2 shows significant differences between patient groups in dynamic parameters $\mathrm{K}_{\mathrm{i}, \mathrm{NLR}}, \mathrm{K}_{\mathrm{i} \text {,patlak }}$ and $\mathrm{k}_{3} /\left(\mathrm{k}_{2}+\mathrm{k}_{3}\right)$ but not in static parameters (SUV). This can be explained by the significant difference in $\tau^{*}$ between patient groups, which is accounted for in dynamic but not in static analysis. Our results suggest that blood clearance rates in patients suffering from pseudarthrosis is altered and thus dynamic parameters are of possible additional value in the evaluation of such patients. However, further prospective studies in larger and more homogeneous patient groups must be done to confirm these results.

The dynamic parameter $\mathrm{K}_{1}$ has experimentally been shown to relate to blood bone perfusion in a porcine model [32] and used by others in the study of patients with hip and lumbar spine osteoporosis [27]. Recently, in a small study in patients comparing mandibular or hip surgery patients with normal volunteers, Raijmakers et al. [33] showed a low correlation between $\mathrm{K}_{1}$ and bone blood flow as measured by ${ }^{15} \mathrm{O}-\mathrm{H}_{2} \mathrm{O}$ PET. Puri suggested that changes in $k_{3} /\left(k_{2}+k_{3}\right)$ may be the best means of using ${ }^{18} \mathrm{~F}-\mathrm{PET}$ scans to investigate changes in osteoblastic activity [27]. In our study, the parameters $K_{1}, k_{2}, k_{3}$ and $v_{b}$ were not statistically significant different between all control and operated regions. This observation might correspond to the reported decrease of stability of $\mathrm{K}_{1}, \mathrm{k}_{2}$, $\mathrm{k}_{3}$ compared to $\mathrm{K}_{\mathrm{i}}$, NLR $[31,34,35]$. Cook et al. stated that due to possible limitations of the model, the physiological significance of parameters $k_{2}$ and $k_{3}$ is not meaningful in relation to the mineralized skeleton [35]. Our study suggests that $k_{3} /\left(k_{2}+k_{3}\right)$, the parameter that represents the portion ${ }^{18} \mathrm{~F}$-fluoride that binds to the mineral after entering the unbound compartment, is also a stable parameter with statistical significance between control and operated ROIs of 0.0030-0.016 in Table 1 as well as in the intervertebral disc space between pseudarthrosis and fused patients $(p=0.016)$ in Table $2 . K_{1} / k_{2}$ could be interesting as well with a large effect size and statistical significance-between control and operated ROIs of 0.0040 0.030 in Table 1 . The ability to separately evaluate $K_{1}, K_{1} / k_{2}$ and $k_{3} /\left(k_{2}+k_{3}\right)$ allows one to distinguish between different biological processes in a ROI. Separate parameters related to blood flow and to osteoblastic activity, can be of great value in the early assessment 
of patients after PLIF surgery. Future studies have to show whether these differences in dynamic parameters are clinically relevant in particular patient groups other than the spinal surgery patients used in this study.

The study has a number of limitations. Dynamic scanning was performed for only 30 minutes, while several other ${ }^{18} \mathrm{~F}$-fluoride dynamic studies scanned for 60 minutes [22, 33, 36]. However, such a long protocol was not feasible in these patients with considerable degree of back pain. Since our scan protocol included a dynamic and a static scan in between which the patient left the scanner, the regions drawn in the dynamic and static scan did not coincide perfectly, which influenced the observed difference in correlation coefficient of $\mathrm{K}_{\mathrm{i}, \mathrm{NLR}}$ and SUV mean60 as compared to $\mathrm{K}_{\mathrm{i}, \mathrm{NLR}}$ and $\mathrm{SU} \mathrm{V}_{\text {mean30. This }}$ also resulted in a larger $95 \% \mathrm{Cl}$ in the Bland Altman plot of SUV mean60 and SUV mean60,2TCM as compared to the plot of SUV mean30 and SUV mean30,2TCM. Therefore, $K_{i, N L R}$ was also compared to SUV at 30 minutes since in this comparison the exact same ROls were used. However, our objective was not to compare both time intervals, but to test the feasibility of a relatively short image acquisition in view of patient comfort, clinical applicability and patient throughput. Indeed, given the rapid kinetics of ${ }^{18}$ F-fluoride [37], other authors [21] have previously shown that it is possible to estimate $\mathrm{K}_{\mathrm{i}, \mathrm{NLR}}$ from a 4-min static scan of the lumbar spine between 30 and 60 minutes together with 2-4 venous blood samples, providing errors relative to the Patlak values of $+0.6 \%$ at 30 min after injection, increasing up to $-3.3 \%$ at $60 \mathrm{~min}$. Although this is a different clinical approach, the present results show correlations of parameters similar to values reported in other studies, indicating that dynamic scanning for 30 minutes may be sufficient. Furthermore, the time interval between PLIF surgery and PET/CT was variable. However, inclusion was performed on the basis of clinical ground, i.e. persistent back pain after lumbar surgery. Besides, for the aim of this study, to compare the static and dynamic analysis methods, this was not an issue. Our analysis approach involved the use of an IDIF instead of the gold standard of arterial sampling. The use of an IDIF in the aorta to obtain bone metabolism values in the spine has been performed before and was validated against arterial sampling [38].

To conclude, this study shows the feasibility of a 30 minute dynamic ${ }^{18} \mathrm{~F}$-fluoride PET/CT scanning and this may provide dynamic parameters clinically relevant to the diagnosis of pseudarthrosis. 
APPENDIX. Analytical solution for time-dependent tissue radioactivity concentration.

NLR analysis was based on the irreversible 2TCM of Hawkins [23] with three parameters and a blood volume fraction (Figure 6).

The measured radioactivity concentration can be calculated from the dynamic parameters, since $C_{T}$ in Figure 6 represents the radioactivity concentration measured by the PETscan. Solving the system of differential equations (4), yields expressions for the radioactivity concentration in the compartments $C_{F}$ and $C_{B}$ which can be combined into an expression for $C_{T}(5)$ that can be deduced from Figure 6 .

$$
\begin{aligned}
& \left\{\begin{array}{l}
\frac{\mathrm{d} C_{F}(t)}{\mathrm{d} t}=K_{1} C_{A}(t)-\left(k_{2}+k_{3}\right) C_{F}(t) \\
\frac{\mathrm{d} C_{B}(t)}{\mathrm{d} t}=k_{3} C_{F}(t)
\end{array}\right. \\
& C_{T}(t)=C_{F}(t)+C_{B}(t)=\left[K_{1} \cdot \mathrm{e}^{-\left(k_{2}+k_{3}\right) t}+K_{i, N L R}\left(1-\mathrm{e}^{-\left(k_{2}+k_{3}\right) t}\right)\right] \otimes C_{A}(t)
\end{aligned}
$$

Correcting $C_{T}$ for the body weight and injected dose, yields SUV exact (6).

$$
S U V_{\text {exact }}=\frac{C_{T}(t)}{I D / m}=\frac{\left[K_{1} \cdot \mathrm{e}^{-\left(k_{2}+k_{3}\right) t}+K_{i, N L R}\left(1-\mathrm{e}^{-\left(k_{2}+k_{3}\right) t}\right)\right] \otimes C_{A}(t)}{I D / m}
$$

When t approaches infinity, this expression can be simplified to (7).

$$
\lim _{t \rightarrow \infty} S U V_{2 T C M} \approx \frac{K_{i, N L R} \cdot \int_{0}^{t} C_{A}(t) \mathrm{d} t}{I D / m}
$$

Whether the exact relation (6) or the simplification (7) was used to calculate SUV mean,2TCM is dependent on whether the assumption of time goes to infinity applied to the particular time point.

The arterial input concentration $\left(C_{A}\right)$ is known for the first 30 minutes from the dynamic scan. To obtain the arterial input concentration at later time points, the $\mathrm{C}_{\mathrm{A}}$-curve was extrapolated with an exponential function from the peak of the $\mathrm{C}_{\mathrm{A}}$-curve of 0-30 minutes.

The factor between SUV and $\mathrm{K}_{\mathrm{i}}$, NLR, can also be written as physically interpretable terms (8).

$$
\lim _{t \rightarrow \infty} S U V_{2 T C M}=K_{i, N L R} \cdot \frac{\int A(t) \mathrm{d} t}{A_{0}} \cdot \frac{m}{V_{\mathrm{b}}}=K_{i, N L R} \cdot \tau \cdot \frac{1}{f_{\mathrm{b}}}=K_{i, N L R} \cdot \tau
$$




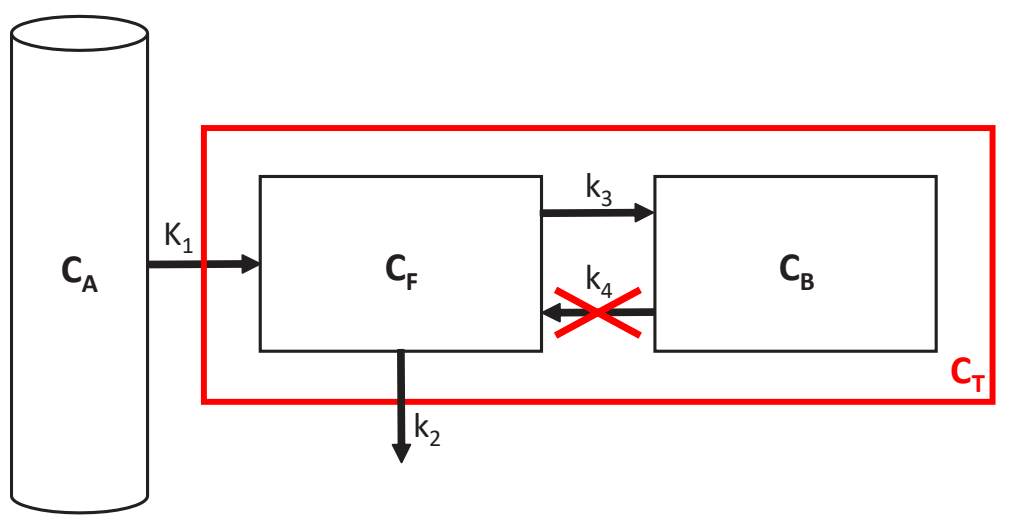

Figure 6. Three-compartment, 4-parameter model for fluoride bone metabolism. Each compartment contains a certain concentration of ${ }^{18} \mathrm{~F}: \mathrm{C}_{\mathrm{A}}[\mathrm{Bq} / \mathrm{ml}]$ is the arterial radioactivity concentration, $\mathrm{C}_{\mathrm{F}}[\mathrm{Bq} / \mathrm{ml}]$ represents the free concentration in the extravascular space unbound to bone and $C_{B}[\mathrm{~Bq} / \mathrm{ml}]$ refers to the radioactivity bound to bone either on the bone surface or fully incorporated in the hydroxyapatite [23]. $\mathrm{C}_{T}[\mathrm{~Bq} / \mathrm{ml}$ represents the total tissue radioactivity concentration that the PET-scanner will measure, which is equal to the summation of $C_{F}$ and $C_{B}$. The rate constants $K_{1}$ to $k_{4}$ describe the transport rate of the fluoride between the compartments. Rate constant $\mathrm{k}_{4}$ can be ignored for this particular situation since the amount of fluoride that will be released after binding to the hydroxyapatite within the time frame of the PET-measurement is negligible. A fractional blood volume parameter, $\mathrm{v}_{\mathrm{b}}[-]$, was also included in the model to account for the plasma and red cell ${ }^{18} \mathrm{~F}-$ fluoride activity in the tissue region. Due to gains and losses from adjacent compartments, the rate of change of tracer concentration in the extravascular space and in the bound compartment can be described. 


\section{REFERENCES}

1. van Tulder MW, Koes BW, Bouter LM. A cost-of-illness study of back pain in The Netherlands. Pain. 1995; 62(2):233-240.

2. Lambeek LC, van Tulder MW, Swinkels IC, Koppes LL, Anema JR, van Mechelen W. The trend in total cost of back pain in The Netherlands in the period 2002 to 2007. Spine. 2011; 36(13):1050-1058.

3. Volinn E. The epidemiology of low back pain in the rest of the world. Spine (Phila Pa 1976). 1997; 22(15):1798.

4. Walker BF. The prevalence of low back pain: a systematic review of the literature from 1966 to 1998. Journal of spinal disorders. 2000; 13(3):205-217.

5. Deyo RA, Bass JE, Walsh NE, Schoenfeld LS, Ramamurthy S. Prognostic variability among chronic pain patients: implications for study design, interpretation, and reporting. Archives of physical medicine and rehabilitation. 1988; 69(3 Pt 1):174-178.

6. Willems PC, Staal JB, Walenkamp GH, de Bie RA. Spinal fusion for chronic low back pain: systematic review on the accuracy of tests for patient selection. The spine journal : official journal of the North American Spine Society. 2013; 13(2):99-109.

7. Larsen JM, Capen DA. Pseudarthrosis of the Lumbar Spine. The Journal of the American Academy of Orthopaedic Surgeons. 1997; 5(3):153-162.

8. Turner JA, Ersek M, Herron L, Haselkorn J, Kent D, Ciol MA, et al. Patient outcomes after lumbar spinal fusions. JAMA : the journal of the American Medical Association. 1992; 268(7):907-911.

9. Raizman NM, O'Brien JR, Poehling-Monaghan KL, Yu WD. Pseudarthrosis of the spine. The Journal of the American Academy of Orthopaedic Surgeons. 2009; 17(8):494-503.

10. Madanat R, Moritz N, Larsson S, Aro HT. RSA applications in monitoring of fracture healing in clinical trials. Scandinavian journal of surgery : SJS : official organ for the Finnish Surgical Society and the Scandinavian Surgical Society. 2006; 95(2):119-127.

11. Ploumis A, Mehbod A, Garvey T, Gilbert T, Transfeldt E, Wood K. Prospective assessment of cervical fusion status: plain radiographs versus CT-scan. Acta orthopaedica Belgica. 2006; 72(3):342-346.

12. Brodsky AE, Kovalsky ES, Khalil MA. Correlation of radiologic assessment of lumbar spine fusions with surgical exploration. Spine. 1991; 16(6 Suppl):S261-265.

13. Carreon LY, Djurasovic M, Glassman SD, Sailer P. Diagnostic accuracy and reliability of fine-cut CT scans with reconstructions to determine the status of an instrumented posterolateral fusion with surgical exploration as reference standard. Spine. 2007; 32(8):892-895.

14. Cleveland M, Bosworth DM, Thompson FR. Pseudarthrosis in the lumbosacral spine. The Journal of bone and joint surgery American volume. 1948; 30A(2):302-312.

15. Hilibrand AS, Dina TS. The use of diagnostic imaging to assess spinal arthrodesis. The Orthopedic clinics of North America. 1998; 29(4):591-601.

16. Gamie S, El-Maghraby T. The role of PET/CT in evaluation of Facet and Disc abnormalities in patients with low back pain using (18)F-Fluoride. Nuclear medicine review Central \& Eastern Europe. 2008; 11(1):1721.

17. Fischer DR, Zweifel K, Treyer V, Hesselmann R, Johayem A, Stumpe KD, et al. Assessment of successful incorporation of cages after cervical or lumbar intercorporal fusion with [(18)F]fluoride positron-emission tomography/computed tomography. European spine journal : official publication of the European Spine 
Society, the European Spinal Deformity Society, and the European Section of the Cervical Spine Research Society. 2011; 20(4):640-648.

18. Brans B, Weijers R, Halders S, Wierts R, Peters M, Punt I, et al. Assessment of bone graft incorporation by $18 \mathrm{~F}$-fluoride positron-emission tomography/computed tomography in patients with persisting symptoms after posterior lumbar interbody fusion. EJNMMI research. 2012; 2(1):42.

19. Quon A, Dodd R, lagaru A, de Abreu MR, Hennemann S, Alves Neto JM, et al. Initial investigation of (1)(8)F$\mathrm{NaF} \mathrm{PET} / \mathrm{CT}$ for identification of vertebral sites amenable to surgical revision after spinal fusion surgery. European journal of nuclear medicine and molecular imaging. 2012; 39(11):1737-1744.

20. Keyes JW, Jr. SUV: standard uptake or silly useless value? Journal of nuclear medicine : official publication, Society of Nuclear Medicine. 1995; 36(10):1836-1839.

21. Siddique M, Blake GM, Frost ML, Moore AE, Puri T, Marsden PK, et al. Estimation of regional bone metabolism from whole-body 18F-fluoride PET static images. European journal of nuclear medicine and molecular imaging. 2012; 39(2):337-343.

22. Blake GM, Siddique M, Frost ML, Moore AE, Fogelman I. Radionuclide studies of bone metabolism: do bone uptake and bone plasma clearance provide equivalent measurements of bone turnover? Bone. 2011; 49(3):537-542.

23. Hawkins RA, Choi Y, Huang SC, Hoh CK, Dahlbom M, Schiepers C, et al. Evaluation of the skeletal kinetics of fluorine-18-fluoride ion with PET. Journal of nuclear medicine : official publication, Society of Nuclear Medicine. 1992; 33(5):633-642.

24. Patlak CS, Blasberg RG. Graphical evaluation of blood-to-brain transfer constants from multiple-time uptake data. Generalizations. Journal of cerebral blood flow and metabolism : official journal of the International Society of Cerebral Blood Flow and Metabolism. 1985; 5(4):584-590.

25. Patlak CS, Blasberg RG, Fenstermacher JD. Graphical evaluation of blood-to-brain transfer constants from multiple-time uptake data. Journal of cerebral blood flow and metabolism : official journal of the International Society of Cerebral Blood Flow and Metabolism. 1983; 3(1):1-7.

26. Frost ML, Blake GM, Cook GJ, Marsden PK, Fogelman I. Differences in regional bone perfusion and turnover between lumbar spine and distal humerus: (18)F-fluoride PET study of treatment-naive and treated postmenopausal women. Bone. 2009; 45(5):942-948.

27. Puri T, Frost ML, Curran KM, Siddique M, Moore AE, Cook GJ, et al. Differences in regional bone metabolism at the spine and hip: a quantitative study using (18)F-fluoride positron emission tomography. Osteoporosis international : a journal established as result of cooperation between the European Foundation for Osteoporosis and the National Osteoporosis Foundation of the USA. 2013; 24(2):633-639.

28. Berman M. Kinetic models for absorbed dose calculations. Pamphlet No. 12. In: National Cancer Institute $\mathrm{NloH}$, Bethesda, Maryland, ed. 1977.

29. Bland JM, Altman DG. Statistical methods for assessing agreement between two methods of clinical measurement. Lancet. 1986; 1(8476):307-310.

30. Siddique M, Frost ML, Blake GM, Moore AE, Al-Beyatti Y, Marsden PK, et al. The precision and sensitivity of (18)F-fluoride PET for measuring regional bone metabolism: a comparison of quantification methods. Journal of nuclear medicine : official publication, Society of Nuclear Medicine. 2011; 52(11):1748-1755.

31. Brenner W, Vernon C, Muzi M, Mankoff DA, Link JM, Conrad EU, et al. Comparison of different quantitative approaches to $18 \mathrm{~F}$-fluoride PET scans. Journal of nuclear medicine : official publication, Society of Nuclear Medicine. 2004; 45(9):1493-1500. 
32. Piert M, Zittel TT, Becker GA, Jahn M, Stahlschmidt A, Maier G, et al. Assessment of porcine bone metabolism by dynamic. Journal of nuclear medicine : official publication, Society of Nuclear Medicine. 2001; 42(7):1091-1100.

33. Raijmakers P, Temmerman OP, Saridin CP, Heyligers IC, Becking AG, van Lingen A, et al. Quantification of 18F-Fluoride Kinetics: Evaluation of Simplified Methods. Journal of nuclear medicine : official publication, Society of Nuclear Medicine. 2014; 55(7):1122-1127.

34. Simoncic $U$, Jeraj R. Cumulative input function method for linear compartmental models and spectral analysis in PET. Journal of Cerebral Blood Flow \& Metabolism. 2010; 31(2):750-756.

35. Cook GJ, Lodge MA, Marsden PK, Dynes A, Fogelman I. Non-invasive assessment of skeletal kinetics using fluorine-18 fluoride positron emission tomography: evaluation of image and population-derived arterial input functions. European journal of nuclear medicine. 1999; 26(11):1424-1429.

36. Puri T, Blake GM, Frost ML, Siddique M, Moore AE, Marsden PK, et al. Comparison of six quantitative methods for the measurement of bone turnover at the hip and lumbar spine using 18F-fluoride PET-CT. Nucl Med Commun. 2012; 33(6):597-606.

37. Zanoli G, Stromqvist B, Jonsson B. Visual analog scales for interpretation of back and leg pain intensity in patients operated for degenerative lumbar spine disorders. Spine. 2001; 26(21):2375-2380.

38. Puri T, Blake GM, Siddique M, Frost ML, Cook GJ, Marsden PK, et al. Validation of new image-derived arterial input functions at the aorta using 18F-fluoride positron emission tomography. Nucl Med Commun. 2011; 32(6):486-495. 


\section{CHAPTER 6}

${ }^{18}$ F-fluoride PET/CT as an early predictor of bony fusion after posterior lumbar interbody fusion a prospective study

Marloes JM Peters, Boudewijn T Brans, Roel Wierts, Elisabeth MC Jutten, Wouter AM Broos, Mariëlle GW Visser, Mariel P ter Laak, Felix M Mottaghy, René E Weijers, Paul C Willems 


\section{ABSTRACT}

\section{BACKGROUND TEXT}

Posterior Lumbar Interbody Fusion (PLIF) is a surgical procedure in which stabilization of spinal segments is achieved by means of pedicle screw instrumentation and interbody cages filled with bone graft. Commonly used follow-up modalities including plain radiography and computed tomography (CT) are of limited use in the early postoperative phase. Positron Emission Tomography (PET) is a nuclear imaging modality that is able to assess physiological processes at the cellular and molecular level, well before manifestation of morphological changes.

\section{PURPOSE}

The goal was to determine whether ${ }^{18} \mathrm{~F}$-fluoride PET/CT findings six weeks after posterior lumbar interbody fusion (PLIF), can predict bony fusion one year postoperatively on CT.

\section{DESIGN}

Prospective study design

\section{PATIENT SAMPLE}

Twenty consecutive patients who underwent PLIF (21 levels) were prospectively included between October 2011 and January 2014 after written informed consent.

\section{OUTCOME MEASURES}

Physiologic measures included PET/CT scanning and diagnostic CT scanning. PET/CT outcome measures: overall bone metabolism, bone blood flow and bone mineral incorporation of the operated segment. CT outcome measure: the amount of bony bridges present between the fused vertebrae.

\section{METHODS}

Based on diagnostic CT one year postoperatively, operated segments were classified into a pseudarthrosis or a fusion group. Dynamic and static ${ }^{18} \mathrm{~F}$-fluoride PET/CT scanning was performed at six weeks and one year, yielding parameters related to overall bone metabolism (SUV max, $\left.K_{i}\right)$, bone blood flow $\left(K_{1} / k_{2}\right)$ and bone mineral incorporation $\left(k_{3} /\left(k_{2}+k_{3}\right)\right)$. Differences in PET/CT parameters between groups and between follow-up moments were assessed. The area under the curve $(A \cup C)$ from the receiver operating characteristic (ROC) was calculated for each PET/CT parameter as a measure of diagnostic accuracy. The costs of the PET/CT scans were partially financed by an External Research Program grant from Medtronic. The authors have no potential conflicts of interest to declare.

\section{RESULTS}

Eleven segments were classified as pseudarthrotic and ten as fused on diagnostic CT. Pseudarthrotic segments showed lower intervertebral overall bone metabolism values compared to fused segments at six weeks after surgery $\left(S U V_{\max }=13.47 \pm 5.88, S U V_{\max }\right.$ $=22.59 \pm 6.42$ respectively, $p=0.005$ ). Furthermore, pseudarthrotic segments compared to 
fused segments showed lower intervertebral bone blood flow at six weeks $\left(\mathrm{K}_{1} / \mathrm{k}_{2}=0.82 \pm 0.47, \mathrm{~K}_{1} / \mathrm{k}_{2}=1.66 \pm 1.11\right.$ respectively, $\left.\mathrm{p}=0.030\right)$ and lower intervertebral bone mineral incorporation at one year $\left(k_{3} /\left(k_{2}+k_{3}\right)=0.41 \pm 0.13, k_{3} /\left(k_{2}+k_{3}\right)=0.54 \pm 0.14\right.$ respectively, $p=0.043)$. $K_{i}$ of the operated intervertebral disc space at six weeks had the highest diagnostic accuracy for predicting the fusion status at one year ( $A \cup C=0.91$ ), followed by SUV $V_{\max }, \mathrm{K}_{1} / \mathrm{k}_{2}$ and $\mathrm{k}_{3} /\left(\mathrm{k}_{2}+\mathrm{k}_{3}\right)$ (AUC=0.86, 0.83, 0.68 respectively).

\section{CONSLUSIONS}

${ }^{18} \mathrm{~F}$-fluoride PET/CT six weeks after PLIF provides prognostic information on bony fusion at one year. 


\section{INTRODUCTION}

Posterior Lumbar Interbody Fusion (PLIF) is a surgical procedure in which stabilization of spinal segments is achieved by means of pedicle screw instrumentation and interbody cages filled with bone graft. The intended outcome is definite bony fusion of the vertebrae involved. The failure rate of lumbar spinal fusion is reported between $30-46 \%$ [1] and pseudarthrosis or non-union is thought to be the cause of pain in a significant number of PLIF patients with persisting or recurrent symptoms $[2,3]$.

The most commonly used imaging modalities for follow-up of PLIF patients are plain radiography and computed tomography (CT) [4]. Radiography and CT aim to visualize callus formation and bony bridging between the involved vertebrae. Since these morphological signs of fusion occur late in the normal course of bone healing, radiography and CT are of limited use in the early postoperative phase $[5,6]$.

Positron Emission Tomography (PET) is a nuclear imaging modality that is able to assess physiological processes at the cellular and molecular level, well before manifestation of morphological changes [7]. PET/CT with the bone seeking tracer ${ }^{18} \mathrm{~F}$-fluoride enables localization and quantification of bone metabolism. The uptake mechanism of ${ }^{18} \mathrm{~F}$-fluoride is based on ion exchange with the hydroxyl group of hydroxyapatite. The portion of bone crystal that is accessible to blood, is available for ${ }^{18} \mathrm{~F}$-fluoride ion exchange [8]. Increased uptake of fluoride is thus related to an increase in bone blood flow and/or an increase in the exposed bone crystal surface [8, 9].

Besides visual inspection, PET/CT scans can be analyzed by means of full pharmacokinetic modelling based on the Hawkins model [10]. This yields the fluoride bone influx rate $K_{i}$ which correlates with histomorphometric parameters of bone formation [11, 12], as well as parameters related to regional bone blood flow and bone mineral incorporation [13]/osteoblastic activity [14, 15]. Full pharmacokinetic modelling requires dynamic scanning. As an alternative, the standardized uptake value (SUV) can be used, for which static scanning suffices. SUV is a semi-quantitative measure for bone metabolism that is more easily obtained. However, SUV is affected by tracer competition and blood clearance, while $K_{i}$ is not [16].

We hypothesize that bone metabolism parameters obtained from ${ }^{18} \mathrm{~F}$-fluoride PET/CT scans early after PLIF, can provide prognostic information on the fusion outcome later on. The purpose of this study was to determine whether ${ }^{18} \mathrm{~F}$-fluoride PET/CT findings six weeks after PLIF can predict bony fusion one year postoperatively on CT. 


\section{MATERIAL AND METHODS}

\section{Patients}

A consecutive cohort of twenty patients was prospectively enrolled at the Maastricht University Medical Center between October 2011 and January 2014, after screening 86 potentially eligible patients (Figure 1). Table 1 shows the patient demographics. One patient underwent PLIF at two levels. Therefore, the number of operated segments was 21. PLIF was performed according to clinical practice as previously described [16, 17]. Dynamic and static ${ }^{18} \mathrm{~F}$-fluoride PET/CT scans were made at six weeks and one year postoperatively as part of the study protocol. This study was performed in accordance with the Helsinki Declaration of 1975, as revised in 2013. The study has been approved by the medical ethical committee of the Maastricht University Medical Center (NL.32881.068.11) and all subjects signed an informed consent form.

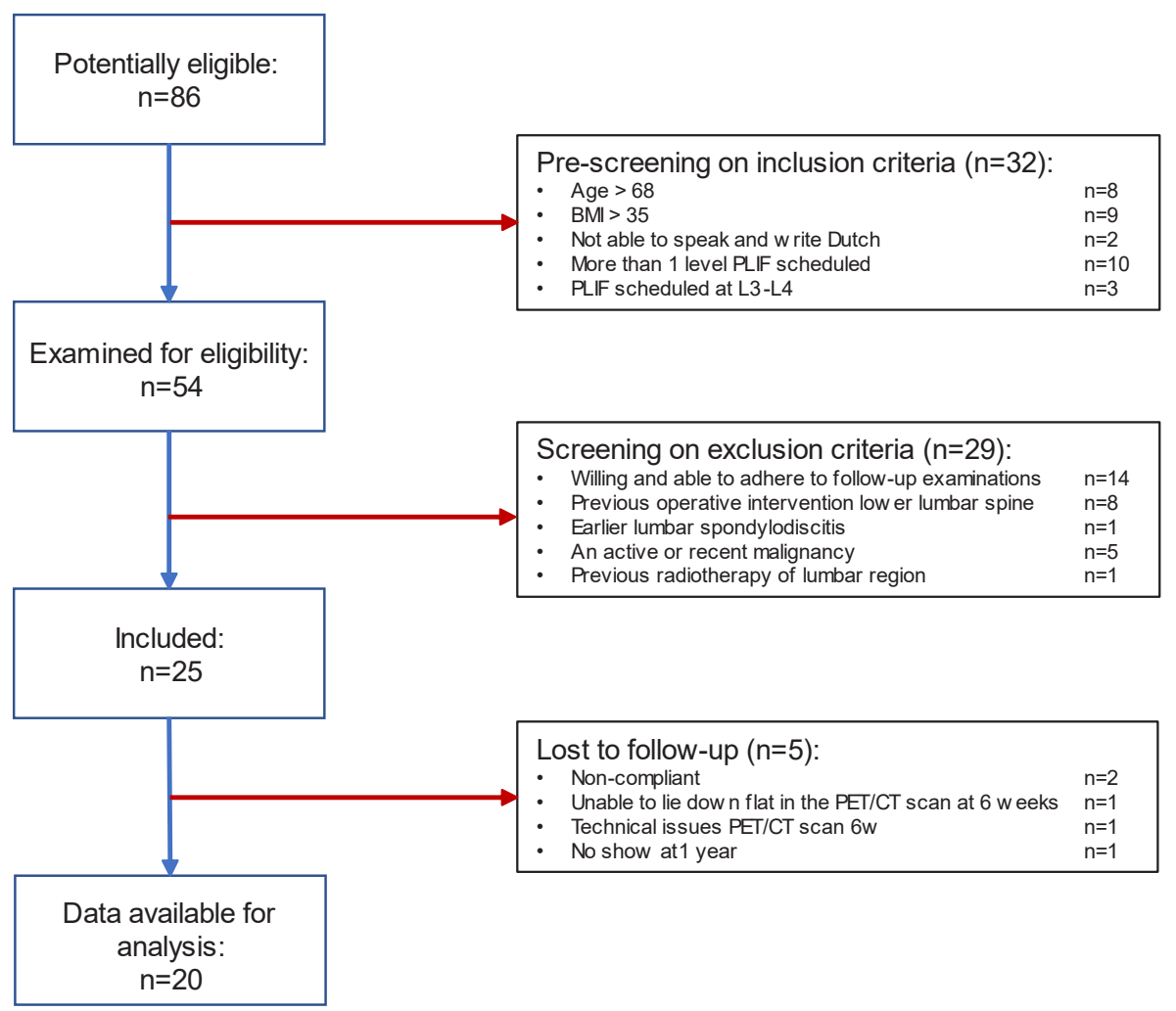

Figure 1. Flow diagram of the patient selection process indicating reasons and numbers for non-participation at each stage. 86 patients were considered potentially eligible. After pre-screening based on inclusion criteria, the 54 remaining patients were further examined for eligibility by screening based on exclusion criteria. 25 patients were included in the study of which twenty completed the study protocol. 
Table 1. Patient demographics.

\begin{tabular}{ll}
\hline Gender: & male $(\mathrm{n}=13)$, female $(\mathrm{n}=7)$ \\
\hline Age at surgery*: & $51.7 \pm 13.1$ years $(18-67)$ \\
\hline BMI*: & $27.2 \pm 2.67 \mathrm{~kg} / \mathrm{m}^{2}(21.6-31.3)$ \\
\hline Indication: & Low grade lumbar spondylolisthesis $(\mathrm{n}=20)$ \\
\hline Level operated: & L4-L5 ( $\mathrm{n}=9), \mathrm{L}$-S1 $(\mathrm{n}=12)$ \\
\hline Time intervals*: & $6.4 \pm 0.62$ weeks $(5.7-8.1)$ \\
- PLIF - early PET/CT (6 weeks) & $53.3 \pm 3.5$ weeks $(47.1-61.1)$ \\
- PLIF - late PET/CT (1 year) & \\
\hline BMI=body mass index & \\
PLIF=posterior lumbar interbody fusion & \\
PET=positron emission tomography & \\
CT=computed tomography & \\
*mean \pm standard deviation (range) &
\end{tabular}

\section{${ }^{18}$ F-fluoride PET/CT scans}

PET and CT images were acquired with an integrated PET/CT scanner (Gemini TF 64 PETCT, Philips, the Netherlands). After a low-dose CT acquisition (120 kV, 30 mAs, slice thickness $4 \mathrm{~mm}$ ), dynamic PET scanning started simultaneously with intravenous injection of $\mathrm{Na}-\left({ }^{18} \mathrm{~F}\right)$-fluoride (mean 194.9 MBq, range 100-221.6 MBq at six weeks and mean 199.9 $\mathrm{MBq}$, range 146-219.8 MBq at one year) and involved a 30-minute list-mode scan of the lower spine ( $18 \mathrm{~cm}$ axial field-of-view). The list-mode scan was rebinned into consecutive time frames: $6 \times 5 \mathrm{~s}, 3 \times 10 \mathrm{~s}, 9 \times 60 \mathrm{~s}, 10 \times 120 \mathrm{~s}$. One hour after tracer administration, another low-dose CT was acquired, followed by a static PET scan of two bed positions lasting five minutes each. PET images were reconstructed into CT based attenuation corrected images. At one year follow-up, a diagnostic CT scan (64-slice helical, 120 kV, 250 mAs, slice thickness $1 \mathrm{~mm}$, increment $0.8 \mathrm{~mm}$ ) was added to the protocol. Scans were viewed on clinical software (EBW, Philips, the Netherlands) and further analyzed on a research tool (PMOD 3.0, PMOD Technologies Ltd, Zürich).

Interbody fusion scoring on CT

Interbody fusion was scored on the diagnostic CT scan at one year postoperatively as either 0,1 or 2, according to the number of bony bridges between the vertebrae of the operated segment (Figure 2) as previously described [17]. A bony bridge was defined as bone mass progressing from one endplate to the other without interruptions. Scoring was performed by three independent blinded observers (experienced musculoskeletal radiologist $\mathrm{RW}$, experienced nuclear medicine physician $\mathrm{BB}$, junior nuclear medicine physician WB). After consensus was reached, segments were classified into either a pseudarthrosis group (score 0 ) or a fusion group (score 1 and 2 ). 


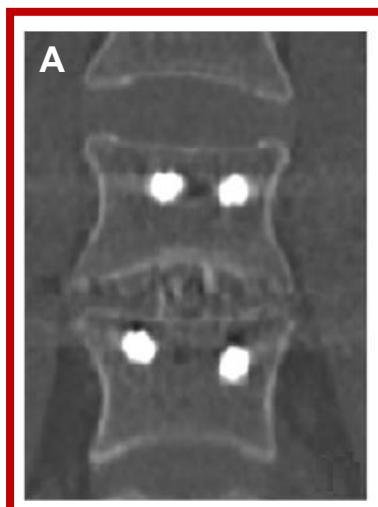

0: no bony bridges

PSEUDARTHROSIS GROUP

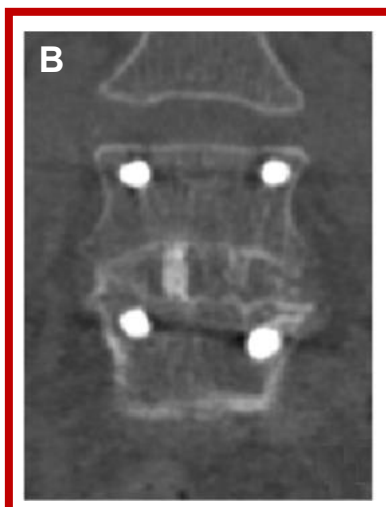

1: one bony bridge

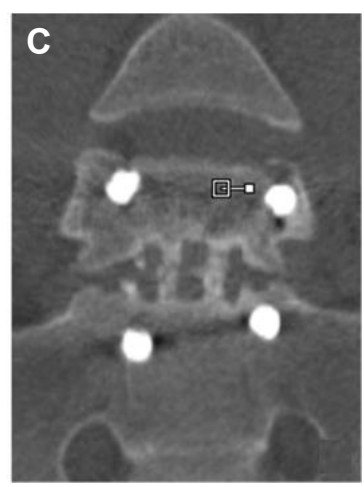

2: $\geq$ two bony bridges

\section{FUSION GROUP}

Figure 2. Examples of interbody fusion scoring on diagnostic CT one year postoperatively and classification into groups. Score 0: no bony bridges (A). Score 1: one complete bony bridge within or surrounding the cages, left or right (B). Score 2: two or more complete bony bridges within or surrounding the cages (C).

\section{Analysis of ${ }^{18} \mathrm{~F}$-fluoride PET/CT scans}

PET/CT parameters were calculated based on a region of interest (ROI) approach as previously reported [16], by two independent and blinded observers (BB, junior researcher with experience in analyzing PET/CT scans MP). For each low-dose CT, six ellipsoid shaped ROIs were manually drawn following the contours of the vertebrae, including the intervertebral disc space and both endplates of the operated segment (referred to as inter, endUP, endLOW) as well as of a control segment two levels above the operated segment (referred to as inter_C, endUP_C, endLOW_C) (Figure 3A).

For static analysis, the ROIs were transferred to the static PET image to obtain the maximum SUV (SUV $\max [-])$ within each ROI (Figure 3B), by correcting the measured radioactivity concentration $(\mathrm{A}[\mathrm{kBq} / \mathrm{ml}])$ for the injected dose of ${ }^{18} \mathrm{~F}$-fluoride (ID [MBq]) and the body weight of the patient $(\mathrm{m}[\mathrm{kg}]$ ) according to (1).

$$
\text { SUVmax }=\max \left(\frac{A}{I D / m}\right)
$$




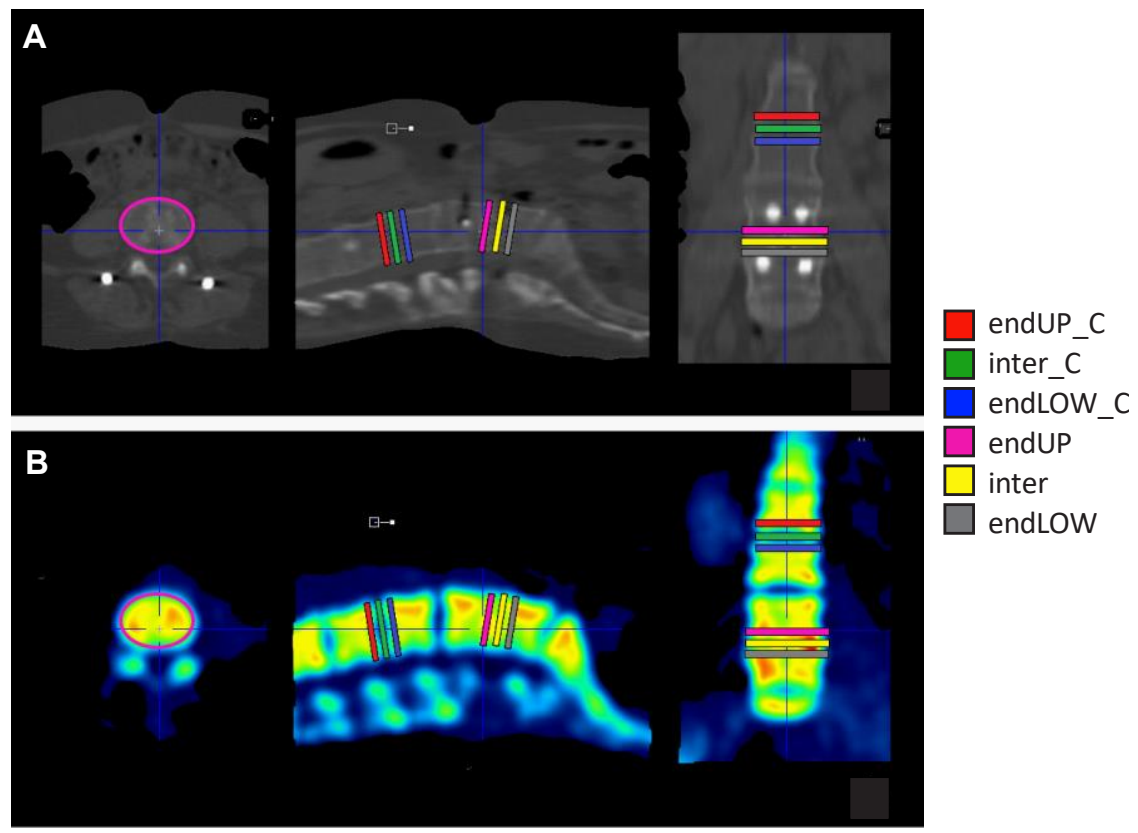

Figure 3. Definition of the six ROIs in PET/CT scan analysis. (A) An axial, sagittal and coronal low-dose CT image (from left to right) of the lower spine after PLIF. Three ROls were drawn in the operated segment: the lower endplate of the cranial vertebra (endUP in pink), the intervertebral disc (inter in yellow), the upper endplate of the caudal vertebra (endLOW in grey). The same three ROls were drawn in a reference segment two levels above the operated segment (endUP_C in red, inter_C in green and endLOW_C in blue, respectively). The ROIs had a slice thickness of $4 \mathrm{~mm}$, a short axis range of 40-50 mm and a long axis range of 55-65 mm. (B) The ROIs were transferred to the co-registered PET image (axial, sagittal, coronal views) and used for analysis of the static and the dynamic scans.

For dynamic analysis, the ROls were applied to the dynamic frames to generate tissue time-activity curves (TACS). The arterial input function was determined by an image derived input function (IDIF) obtained via thresholding [16]. The IDIF and ROI TACs were fitted to the 2-tissue compartment model $[10,16]$, to obtain rate constants $K_{1}$, $k_{2}$ and $k_{3}$ that describe the transport of fluoride between the vascular compartment, the extracellular compartment and the bone mineral compartment. The rate constants were combined into $K_{i}, K_{1} / k_{2}$ and $k_{3} /\left(k_{2}+k_{3}\right)$ for further analysis. $K_{i}$ represents the net transport of ${ }^{18} \mathrm{~F}$-fluoride into bone and is a marker for regional bone metabolism $[10,11,18]$, calculated according to (2).

$$
K_{i}=\frac{K_{1} \cdot k_{3}}{k_{2}+k_{3}}
$$


$\mathrm{K}_{1}$ and $\mathrm{K}_{2}$ describe the forward and reverse capillary transport. $\mathrm{K}_{1}$ is correlated to bone perfusion [12]. $K_{1} / k_{2}$ represents the volume of distribution of tracer in the unbound pool [19] and is related to bone blood flow. $\mathrm{k}_{3}$ describes the binding of ${ }^{18} \mathrm{~F}$-fluoride to the bone matrix [20]. $k_{3} /\left(k_{2}+k_{3}\right)$ represents the fraction of tracer in the extravascular tissue space that undergoes specific binding to the bone mineral [21] and is thus related to bone mineral incorporation.

\section{Statistical analysis}

Statistical evaluation was performed using IBM SPSS Statistics version 23.0 (Armonk, NY: IBM Corporation). Intergroup differences (pseudarthrosis versus fusion) were assessed by an independent t-test in case of normality and a Mann-Whitney U-test otherwise. Intraindividual differences (operated versus non-operated segments, endplate versus intervertebral) and intertime differences (six weeks versus one year) were evaluated by a paired samples t-test in case of normality and a Wilcoxon Signed-Rank test otherwise. Pvalues $\leq 0.05$ were used to indicate statistically significant differences. Receiver Operating Characteristic (ROC) curves were used to test the performance of ${ }^{18} \mathrm{~F}$-fluoride PET/CT parameters at six weeks for the prediction of bony fusion one year postoperative. The area under the curve $(A \cup C)$ was used as accuracy measure covering all possible interpretation thresholds [22]. The Youden Index was used to obtain the cut-point at which each parameter achieved the optimum differentiating ability with an equal weight given to sensitivity and specificity $[23,24]$.

\section{RESULTS}

Interbody fusion scoring on CT

Of the 21 segments evaluated, eleven levels were scored as 0 (no bony bridges), five levels as 1 (one bony bridge), and five levels as 2 (two or more bony bridges) on diagnostic CT one year after PLIF. The eleven levels without bony bridges were classified into the pseudarthrosis group, and the ten levels with one or more bony bridges were classified into the fusion group.

Analysis of ${ }^{18} \mathrm{~F}$-fluoride PET/CT-scans

Analysis of the PET/CT scans yielded four PET/CT parameters for each ROI: SUV $\max$ from the static scan and $K_{i}, K_{1} / k_{2}, k_{3} /\left(k_{2}+k_{3}\right)$ from the dynamic scan. 


\section{Differences between the fusion group and the pseudarthrosis group}

Figure 4 shows the differences in bone metabolic parameters SUV $\max$ and $K_{i}$ between the fusion and the pseudarthrosis group six weeks after PLIF. An overview of p-values for all intergroup and intraindividual differences is provided in Table 2. SUV $\max$ of the operated intervertebral disc space was significantly lower in the pseudarthrosis group compared to the fusion group $\left(S U V_{\max }=13.47 \pm 5.88\right.$ and $S U V_{\text {max }}=22.59 \pm 6.42$ respectively, $p=0.005$ ). Similar results were found for $K_{i}$ of the operated intervertebral disc space $\left(K_{i}=0.060 \pm 0.02\right.$ for the pseudarthrosis group, $K_{i}=0.10 \pm 0.02$ for the fusion group, $p=0.001$ ).

When comparing the operated ROIs to the control ROIs for SUVmax and $\mathrm{K}_{\mathrm{i}}$, significantly higher values were found in all three operated ROls for both the fusion and the pseudarthrosis group. Focusing on the operated segments, the endplates and the intervertebral disc space were equally metabolically active in the fusion group, while in the pseudarthrosis group, the bone metabolic activity of the intervertebral disc space was significantly lower than at the endplates. Focusing on the control segments, the metabolic activity was significantly lower in the intervertebral disc space compared to at the endplates, similar to the trend seen in the operated pseudarthrotic segments.
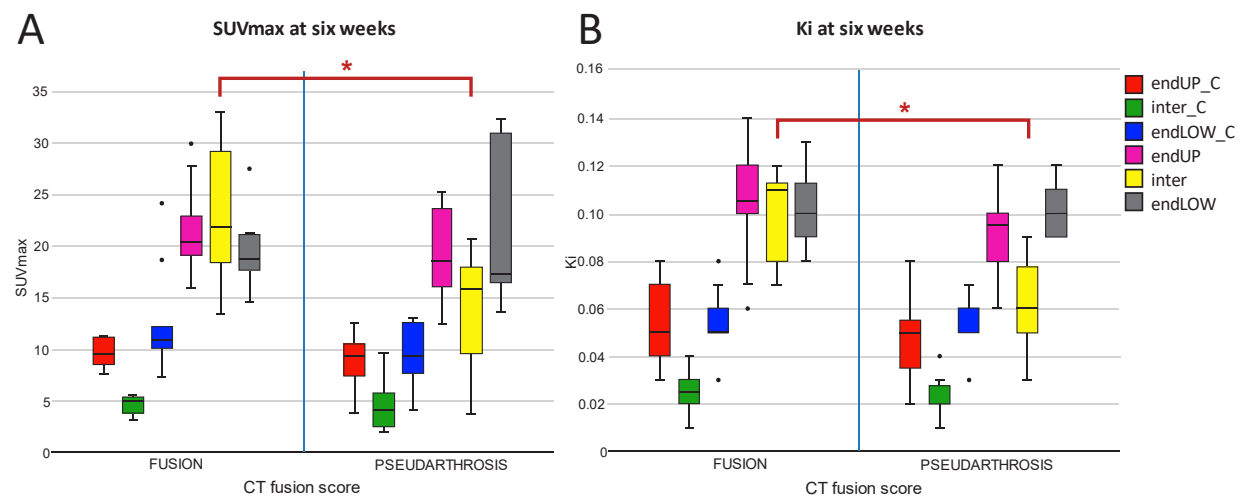

Figure 4. SUV $\max (A)$ and $K_{i}(B)$ at six weeks after PLIF for the fusion group (left from the vertical blue line) and for the pseudarthrosis group (right from the vertical blue line) of the control ROls (endUP_C, inter_C, endLOW_C in red, green and blue respectively) and the operated ROIs (endUP, inter, endLOW in pink, yellow and grey respectively). Statistically significant differences between the groups were depicted by a red asterisk.

Figure 5 shows the intergroup differences between parameters related to bone blood flow $\left(K_{1} / k_{2}\right)$ and bone mineral incorporation $\left(k_{3} /\left(k_{2}+k_{3}\right)\right)$ specifically, at six weeks and one year after PLIF. An overview of the $p$-values for all intergroup and intraindividual differences at six weeks and one year is provided in Table 2 .

At six weeks postoperatively, $\mathrm{K}_{1} / \mathrm{k}_{2}$ in the operated upper endplate and intervertebral disc space was significantly higher in the fusion group compared to the pseudarthrosis group ( $p=0.036$ and $p=0.030$ respectively) (Figure $5 A$ ). No intergroup differences were found for $\mathrm{k}_{3} /\left(\mathrm{k}_{2}+\mathrm{k}_{3}\right)$ (Figure $\left.5 \mathrm{~B}\right)$. 
When comparing the operated ROls to the control ROIs, significantly higher $\mathrm{K}_{1} / \mathrm{k}_{2}$ values were found in all three operated ROls for the fusion group, while in the pseudarthrosis group $k_{1} / k_{2}$ was increased in the operated intervertebral disc space only. $k_{3} /\left(k_{2}+k_{3}\right)$ was significantly higher in all three operated ROls in comparison to the control ROls for both the fusion and the pseudarthrosis group.

At one year postoperatively, no intergroup differences were found for $\mathrm{K}_{1} / \mathrm{k}_{2}$ (Figure $5 \mathrm{C}$ ). $k_{3} /\left(k_{2}+k_{3}\right)$ in the operated intervertebral disc space was significantly lower in the pseudarthrosis group compared to the fusion group ( $p=0.043$ ) (Figure 5D). $k_{1} / k_{2}$ and $k_{3} /\left(k_{2}+k_{3}\right)$ values were significantly higher in all three operated ROls compared to the control ROIs for both the fusion and the pseudarthrosis group, except for $K_{1} / k_{2}$ in the operated lower endplate of the fusion group.

In other words, pseudarthrotic patients exhibit lower bone blood flow towards the operated segment in comparison to fused patients at six weeks postoperatively, which results in less bone mineral incorporation in the intervertebral disc space one year postoperatively.
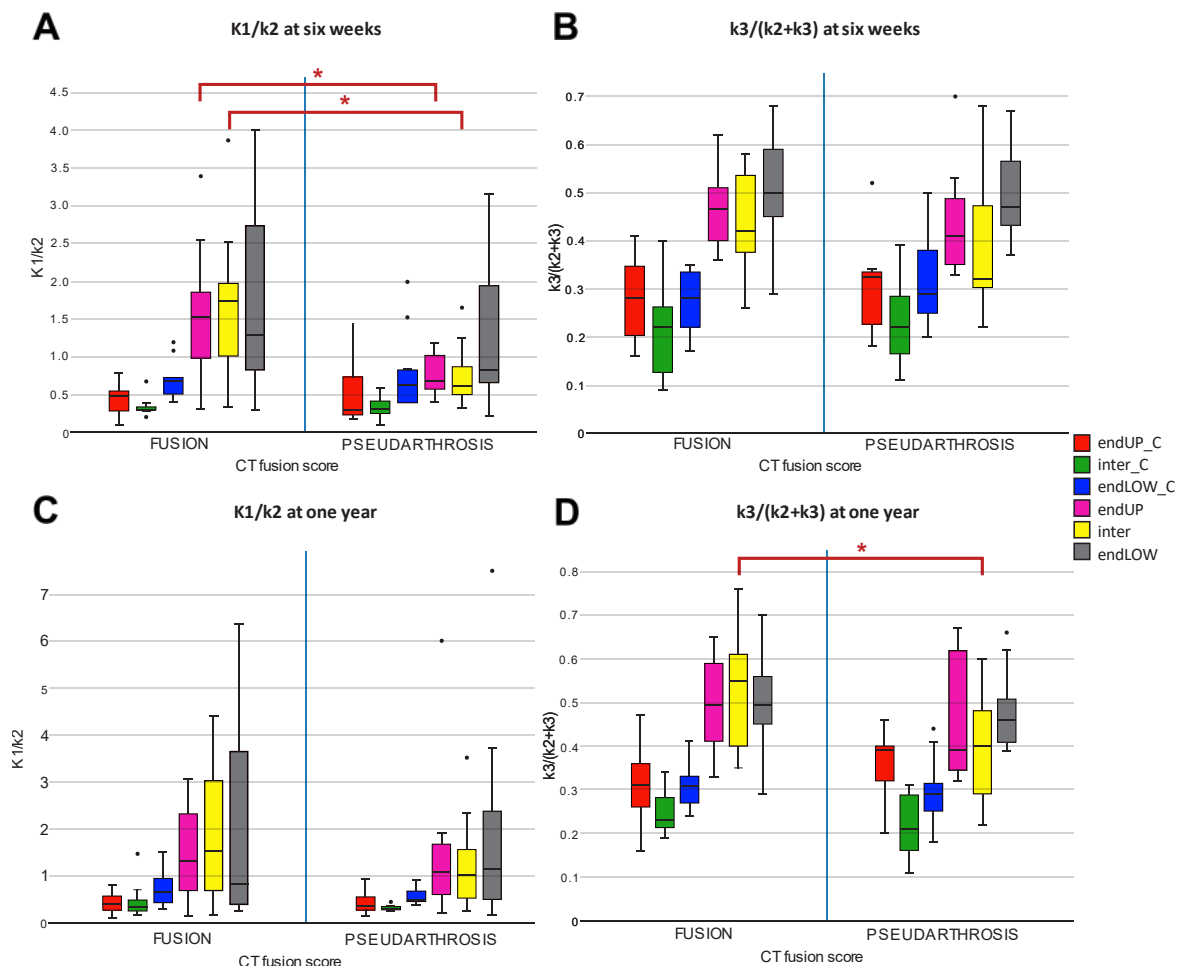

Figure 5. $K_{1} / k_{2}$ and $k_{3} /\left(k_{2}+k_{3}\right)$ at six weeks after PLIF (A and B) and at one year (C and D), for the fusion group (left from the vertical blue line) and for the pseudarthrosis group (right from the vertical blue line) of the control ROIs (endUP_C, inter_C, endLOW_C in red, green and blue respectively) and the operated ROls (endUP, inter, endLOW in pink, yellow and grey respectively). Statistically significant differences between the groups were depicted by a red asterisk. 


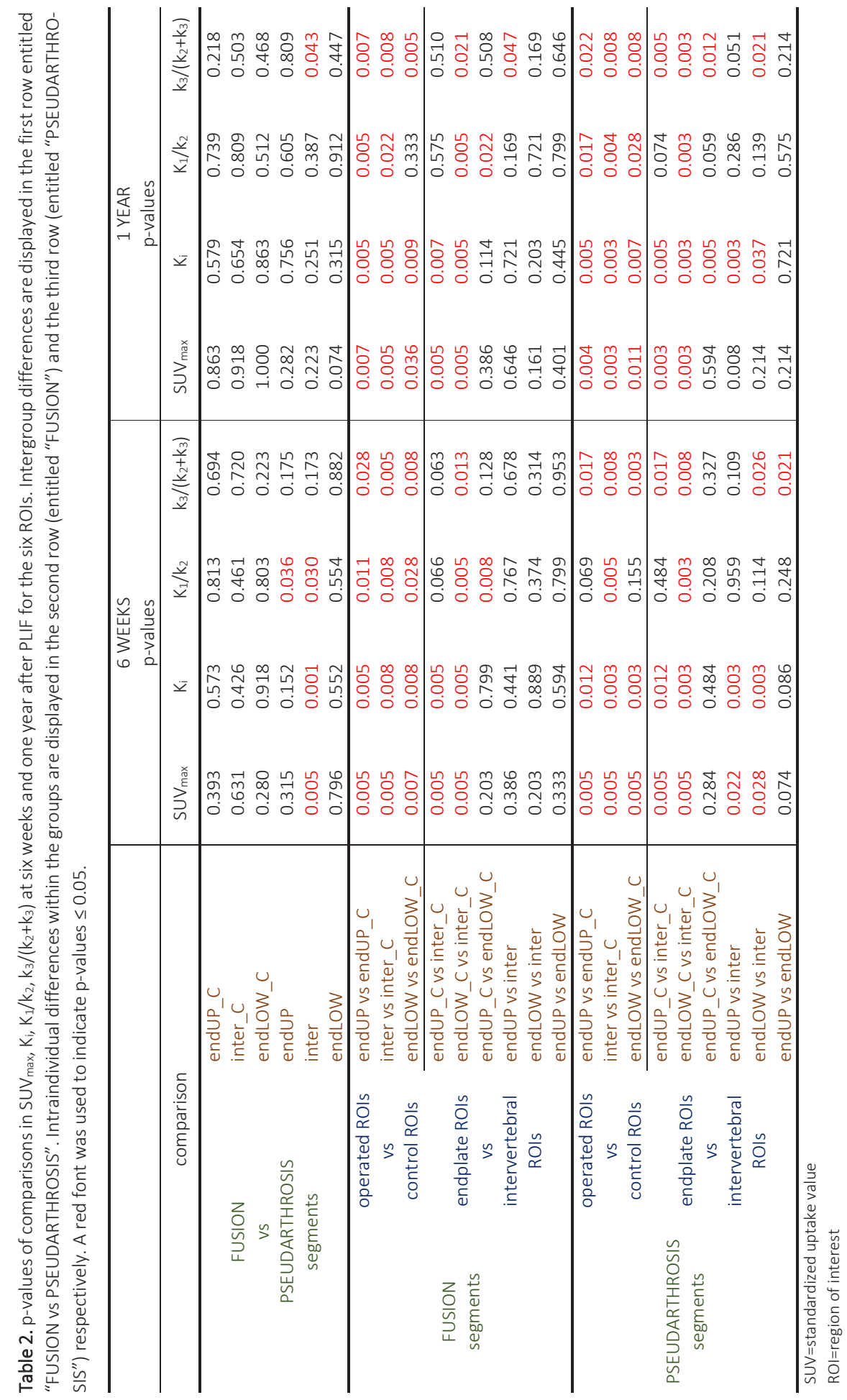




\section{Differences in overall bone metabolism between six weeks and one year}

Figure 6 shows the intertime differences in bone metabolism for both the fusion and the pseudarthrosis group. For the fusion group, a significant decrease in bone metabolic activity over time was observed in the upper endplate $(p=0.005)$, intervertebral disc space $(p=0.005)$ and lower endplate $(p=0.012)$ of the operated segment. For the pseudarthrosis group, a significant decrease in bone metabolic activity over time was observed in the operated lower endplate $(p=0.022)$, but not in the upper endplate $(p=0.575)$ nor in the intervertebral disc space $(p=0.074)$.

For both groups, the operated ROIs one year after PLIF remained significantly higher than the respective control ROIs $(p=0.007, p=0.005, p=0.036$ for the upper endplate, intervertebral disc space, lower endplate respectively of the fused segments and $p=0.004$, $p=0.003, p=0.011$ respectively for the pseudarthrotic segments).
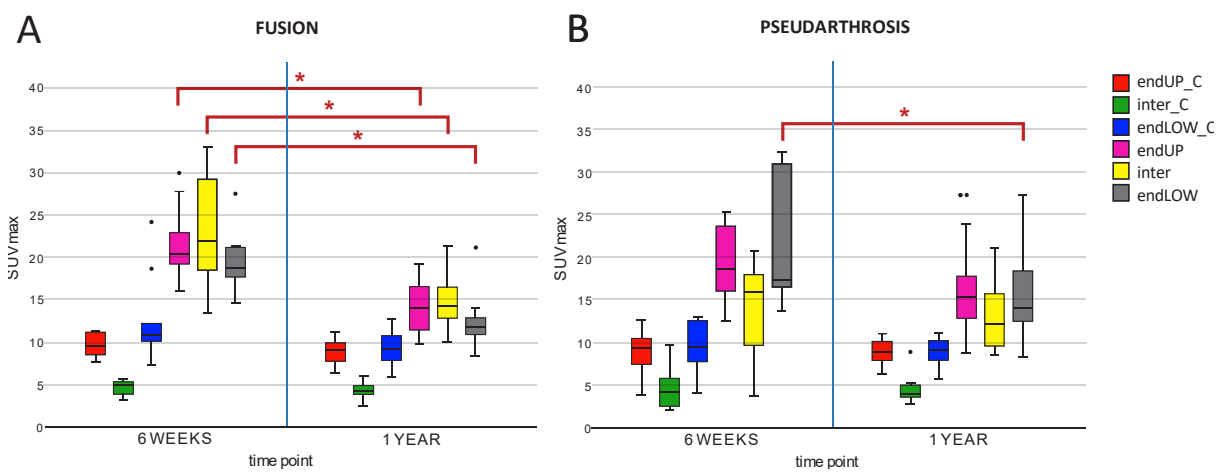

Figure 6. SUV $V_{\max }$ for the fusion group (A) and the pseudarthrosis group (B) at six weeks (left from the vertical blue line) and at one year after PLIF (right from the vertical blue line) of the control ROls (endUP_C, inter_C, endLOW_C in red, green and blue respectively) and the operated ROls (endUP, inter, endLOW in pink, yellow and grey respectively). Statistically significant differences between the groups were depicted by a red asterisk.

\section{ROC curves}

The ROC curves in Figure 7 evaluate the effectiveness of SUV $\max , K_{i}, K_{1} / k_{2}$ and $k_{3} /\left(k_{2}+k_{3}\right)$ in the operated intervertebral disc space at six weeks to predict bony fusion one year after PLIF. With an AUC of 0.91, $\mathrm{K}_{\mathrm{i}}$ had the highest discriminative power, followed by SUV $\max$ $(A \cup C=0.86), K_{1} / k_{2}(A \cup C=0.83)$ and $k_{3} /\left(k_{2}+k_{3}\right)(A \cup C=0.68)$. Optimal cut-points for $S U V_{\max }$ and $K_{i}$ were 18.21 and 0.0750 , resulting in sensitivity values of $0.80,0.90$ and specificity values of $0.89,0.73$ respectively. For $\mathrm{K}_{1} / \mathrm{k}_{2}$ and $\mathrm{k}_{3} /\left(\mathrm{k}_{2}+\mathrm{k}_{3}\right)$, optimal cut-points were 0.8750 and 0.3500 , resulting in sensitivity values of $0.89,0.80$ and specificity values of $0.90,0.64$ respectively. 

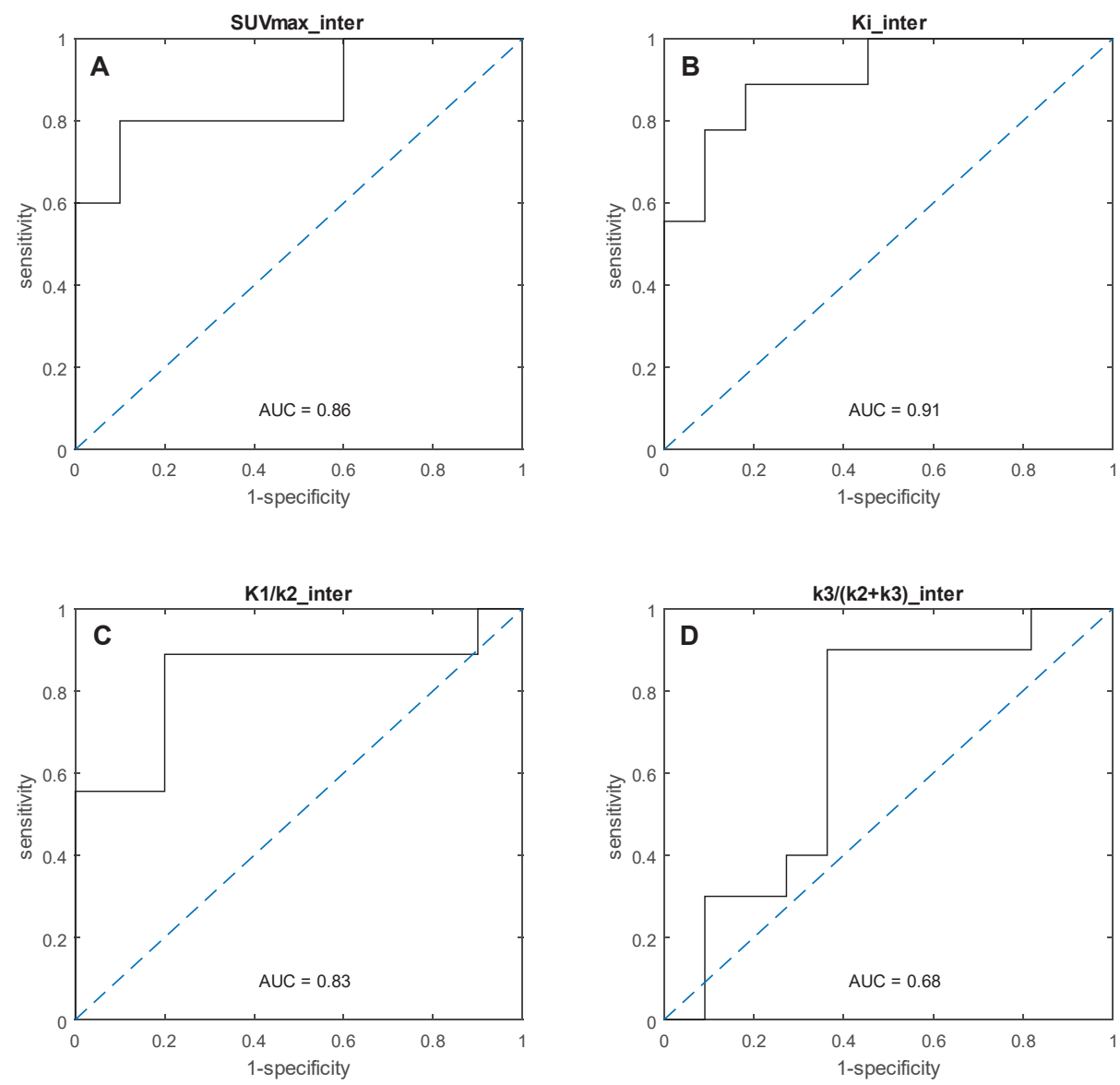

Figure 7. ROC curves for SUV $\max (A), K_{i}(B), K_{1} / k_{2}(C), k_{3} /\left(k_{2}+k_{3}\right)(D)$ in the operated intervertebral disc space at six weeks after PLIF to predict fusion at one year. The area under the curve (AUC) is a measure for the diagnostic accuracy. The diagonal dashed line represents the line of equality.

\section{DISCUSSION}

This prospective study evaluated vertebral bone metabolic activity six weeks and one year after PLIF by means of ${ }^{18} \mathrm{~F}$-fluoride PET/CT in relation to bony fusion on CT one year postoperatively.

The most important finding was that six weeks after PLIF, the bone metabolic activity $\left(S U V_{\max }, K_{i}\right)$ in the operated intervertebral disc space was significantly lower in patients who developed pseudarthrosis as compared to patients who attained solid interbody fusion one year postoperatively. In fused segments, the intervertebral bone metabolic activity equals that of the endplates, while pseudarthrotic segments show a pattern of high 
endplate metabolism and lower intervertebral metabolism, similar to non-operated segments.

Full pharmacokinetic analysis enabled us to show deviating patterns between pseudarthrotic and fused segments in parameters related to bone blood flow $\left(K_{1} / k_{2}\right)$ and bone mineral incorporation $\left(k_{3} /\left(k_{2}+k_{3}\right)\right)$. Pseudarthrotic segments exhibited lower bone blood flow towards the operated segment in comparison to fused segments six weeks postoperatively, which resulted in less bone mineral incorporation in the intervertebral disc space at one year. This may imply that insufficient abrasion of the endplates to enhance blood flow to the bone graft during surgery, which is crucial for bone fusion to develop in a later stage [25], can be detected with ${ }^{18} \mathrm{~F}$-fluoride PET/CT at six weeks postoperatively. Dynamic scanning is inherently restricted to a single bed position and therefore a limited field-of view. At the edges of the field-of-view, the measurement uncertainty is larger as the sensitivity decreases towards the edges [26]. This might explain some unexpected findings in the outer ROIs (endUP_C and endLOW). For example, this might explain why $\mathrm{K}_{1} / \mathrm{K}_{2}$ in the lower endplate at six weeks was not significantly lower in pseudarthrotic segments compared to fused segments (Figure 5A).

At six weeks, SUV max and $K_{i}$ were superior to $K_{1} / k_{2}$ and $k_{3} /\left(k_{2}+k_{3}\right)$ in terms of potential effectiveness in predicting bony fusion (Figure 7). The effectiveness of SUV $V_{\text {max }}$ and $K_{i}$ were similar and therefore SUV $V_{\max }$ could be used in clinical practice for ease of calculation. The more elaborate dynamic modeling technique could be used in research related studies to understand the bone physiology underlying overall bone metabolic changes.

The level of bone metabolism in successfully fused segments decreased from six weeks to one year postoperatively, but was still increased compared to non-operated segments. This is consistent with literature showing elevated bone metabolism values within operated bone regions over a course of several years after surgery $[5,27]$. In contrast, the level of bone metabolism in pseudarthrotic segments remained equally elevated over time. This is in accordance with findings on impaired graft healing of Brenner et al. [5]. Non-decreased levels of bone metabolism at one year can indicate ongoing bony bridging, but other processes such as subsidence can also elevate bone metabolism values [28]. In this study we did not differentiate between these processes.

A strength of this study was that both fused and pseudarthrotic segments were part of the prospective study cohort. This allowed us to follow-up uncomplicated cases and look for deviations in the pseudarthrosis group. Another strength was the addition of pharmacokinetic modelling to the more commonly used SUV calculation. A limitation was that the final diagnosis, classifying segments as either fused or pseudarthrotic, was based on CT one year after PLIF. The diagnostic accuracy of CT to diagnose pseudarthrosis is not perfect [4]. However, in absence of the gold standard surgical exploration, CT imaging appears to be the most reliable noninvasive method to determine fusion after PLIF [3, 4]. Furthermore, bony fusion might occur later than one year postoperatively. Together with the strict definition of a bony bridge that we used, this might explain the high level of 
pseudarthrotic segments observed in this study. An additional follow-up moment several years after surgery would be of value to further differentiate between delayed fusion and definite pseudarthrosis.

Although our results should be confirmed by other studies, the findings are interesting and could be used to base further research upon.

Static PET/CT scanning early after PLIF could be performed in patients that undergo PLIF and are at risk for the development of pseudarthrosis (for example smokers [29] or patients with reduced perfusion). In those cases, aberrant PET/CT findings could hint towards treatment with bone stimulating medication such as novel bone morphogenic proteins, growth hormones, and certain cytokines [30]. Additionally, (dynamic) ${ }^{18} \mathrm{~F}$-fluoride $\mathrm{PET} / \mathrm{CT}$ could be used as a tool to evaluate bone fracture treatment, consolidation of different bone graft materials and ingrowth of coated prostheses and biomaterials that are new on the market.

To conclude, ${ }^{18} \mathrm{~F}$-fluoride PET/CT six weeks after posterior lumbar interbody fusion provides prognostic information on bony fusion at one year. SUV $\max$ of the operated intervertebral disc space obtained early after PLIF could be useful in clinical practice. Differences between fusion groups in bone blood flow and bone mineral incorporation specifically were demonstrated by full pharmacokinetic modelling.

\section{COMPLIANCE WITH ETHICIAL STANDARDS}

Funding: An External Research Program grant from Medtronic partially covered ${ }^{18} \mathrm{~F}$-fluoride $\mathrm{PET} / \mathrm{CT}$ scanning costs. Medtronic was not involved in study execution or data analysis.

Conflict of Interest: The authors have no potential conflicts of interest to declare.

Ethical Approval: All procedures performed in studies involving human participants were in accordance with the ethical standards of the Maastricht University Medical Center (NL.32881.068.11) and with the 1964 Helsinki declaration and its later amendments or comparable ethical standards.

Informed Consent: Informed consent was obtained from all individual participants included in the study. 


\section{REFERENCES}

1. Chan CW, Peng P. Failed back surgery syndrome. Pain medicine (Malden, Mass). 2011; 12(4):577-606.

2. O'Beirne J, O'Neill D, Gallagher J, Williams DH. Spinal fusion for back pain: a clinical and radiological review. Journal of spinal disorders. 1992; 5(1):32-38.

3. Raizman NM, O'Brien JR, Poehling-Monaghan KL, Yu WD. Pseudarthrosis of the spine. The Journal of the American Academy of Orthopaedic Surgeons. 2009; 17(8):494-503.

4. Choudhri TF, Mummaneni PV, Dhall SS, Eck JC, Groff MW, Ghogawala Z, et al. Guideline update for the performance of fusion procedures for degenerative disease of the lumbar spine. Part 4: radiographic assessment of fusion status. Journal of neurosurgery Spine. 2014; 21(1):23-30.

5. Brenner W, Vernon C, Conrad EU, Eary JF. Assessment of the metabolic activity of bone grafts with (18)Ffluoride PET. European journal of nuclear medicine and molecular imaging. 2004; 31(9):1291-1298.

6. Palestro CJ. Radionuclide imaging after skeletal interventional procedures. Seminars in nuclear medicine. 1995; 25(1):3-14.

7. Beheshti M, Mottaghy FM, Payche F, Behrendt FF, Van den Wyngaert T, Fogelman I, et al. (18)F-NaF $\mathrm{PET} / \mathrm{CT}$ : EANM procedure guidelines for bone imaging. European journal of nuclear medicine and molecular imaging. 2015; 42(11):1767-1777.

8. Blau M, Ganatra R, Bender MA. 18 F-fluoride for bone imaging. Seminars in nuclear medicine. 1972; 2(1):31-37.

9. Segall G, Delbeke D, Stabin MG, Even-Sapir E, Fair J, Sajdak R, et al. SNM practice guideline for sodium 18Ffluoride PET/CT bone scans 1.0. Journal of nuclear medicine : official publication, Society of Nuclear Medicine. 2010; 51(11):1813-1820.

10. Hawkins RA, Choi Y, Huang SC, Hoh CK, Dahlbom M, Schiepers C, et al. Evaluation of the skeletal kinetics of fluorine-18-fluoride ion with PET. Journal of nuclear medicine : official publication, Society of Nuclear Medicine. 1992; 33(5):633-642.

11. Messa C, Goodman WG, Hoh CK, Choi Y, Nissenson AR, Salusky IB, et al. Bone metabolic activity measured with positron emission tomography and [18F]fluoride ion in renal osteodystrophy: correlation with bone histomorphometry. The Journal of clinical endocrinology and metabolism. 1993; 77(4):949-955.

12. Piert M, Zittel TT, Machulla HJ, Becker GA, Jahn M, Maier G, et al. Blood flow measurements with [(15)O]H2O and [18F]fluoride ion PET in porcine vertebrae. Journal of bone and mineral research : the official journal of the American Society for Bone and Mineral Research. 1998; 13(8):1328-1336.

13. Frost ML, Fogelman I, Blake GM, Marsden PK, Cook G, Jr. Dissociation between global markers of bone formation and direct measurement of spinal bone formation in osteoporosis. Journal of bone and mineral research : the official journal of the American Society for Bone and Mineral Research. 2004; 19(11):17971804.

14. Raijmakers $P$, Temmerman $O P$, Saridin CP, Heyligers IC, Becking AG, van Lingen A, et al. Quantification of 18F-Fluoride Kinetics: Evaluation of Simplified Methods. Journal of nuclear medicine : official publication, Society of Nuclear Medicine. 2014; 55(7):1122-1127.

15. Schliephake H, Berding G, Knapp WH, Sewilam S. Monitoring of graft perfusion and osteoblast activity in revascularised fibula segments using [18F]-positron emission tomography. International journal of oral and maxillofacial surgery. 1999; 28(5):349-355. 
16. Peters MJ, Wierts R, Jutten EM, Halders SG, Willems PC, Brans B. Evaluation of a short dynamic 18F-fluoride $\mathrm{PET} / \mathrm{CT}$ scanning method to assess bone metabolic activity in spinal orthopedics. Annals of nuclear medicine. 2015; 29(9):799-809.

17. Peters $M$, Willems $P$, Weijers $R$, Wierts $R$, Jutten $L$, Urbach $C$, et al. Pseudarthrosis after lumbar spinal fusion: the role of (1)(8)F-fluoride PET/CT. European journal of nuclear medicine and molecular imaging. 2015; 42(12):1891-1898.

18. Piert M, Zittel TT, Becker GA, Jahn M, Stahlschmidt A, Maier G, et al. Assessment of porcine bone metabolism by dynamic. Journal of nuclear medicine : official publication, Society of Nuclear Medicine. 2001; 42(7):1091-1100.

19. Puri T, Frost ML, Curran KM, Siddique M, Moore AE, Cook GJ, et al. Differences in regional bone metabolism at the spine and hip: a quantitative study using (18)F-fluoride positron emission tomography. Osteoporosis international : a journal established as result of cooperation between the European Foundation for Osteoporosis and the National Osteoporosis Foundation of the USA. 2013; 24(2):633-639.

20. Wong KK, Piert M. Dynamic bone imaging with 99mTc-labeled diphosphonates and 18F-NaF: mechanisms and applications. Journal of nuclear medicine : official publication, Society of Nuclear Medicine. 2013; 54(4):590-599.

21. Frost ML, Blake GM, Cook GJ, Marsden PK, Fogelman I. Differences in regional bone perfusion and turnover between lumbar spine and distal humerus: (18)F-fluoride PET study of treatment-naive and treated postmenopausal women. Bone. 2009; 45(5):942-948.

22. Eng J. Receiver operating characteristic analysis: a primer. Academic radiology. 2005; 12(7):909-916.

23. Youden WJ. Index for rating diagnostic tests. Cancer. 1950; 3(1):32-35.

24. Ruopp MD, Perkins NJ, Whitcomb BW, Schisterman EF. Youden Index and optimal cut-point estimated from observations affected by a lower limit of detection. Biometrical journal Biometrische Zeitschrift. 2008; 50(3):419-430.

25. Herkowitz HN, Garfin SR, Eismont FJ, Bell GR, Balderston RA. Rothman-Simeone The Spine E-Book: Expert Consult: Elsevier Health Sciences, 2011.

26. Cherry SR, Dahlbom M. PET: physics, instrumentation, and scanners. PET; 2006: Springer; 2006. p. 1-117.

27. Piert M, Winter E, Becker GA, Bilger K, Machulla H, Muller-Schauenburg W, et al. Allogenic bone graft viability after hip revision arthroplasty assessed by dynamic [18F]fluoride ion positron emission tomography. European journal of nuclear medicine. 1999; 26(6):615-624.

28. Brans B, Weijers R, Halders S, Wierts R, Peters M, Punt I, et al. Assessment of bone graft incorporation by $18 \mathrm{~F}$-fluoride positron-emission tomography/computed tomography in patients with persisting symptoms after posterior lumbar interbody fusion. EJNMMI research. 2012; 2(1):42.

29. Hermann PC, Webler M, Bornemann R, Jansen TR, Rommelspacher Y, Sander K, et al. Influence of smoking on spinal fusion after spondylodesis surgery: A comparative clinical study. Technology and health care : official journal of the European Society for Engineering and Medicine. 2016; 24(5):737-744.

30. Dyke JP, Aaron RK. Noninvasive methods of measuring bone blood perfusion. Annals of the New York Academy of Sciences. 2010; 1192:95-102. 


\section{CHAPTER 7}

General Discussion 
$\mathrm{PET} / \mathrm{CT}$ is a hybrid imaging modality. The term hybrid imaging refers to the combination of two imaging modalities that complement each other to create new imaging opportunities. In PET/CT, CT provides three-dimensional detailed images of organs, bones and soft tissue in a patient. On the other hand, PET is a functional modality that provides insight in cellular and molecular processes. The strength of PET/CT lies in its ability to localize the activity of cellular and molecular processes. Considering the manifold different available tracers for PET imaging, the applications of PET/CT are numerous. When using the tracer ${ }^{18} \mathrm{~F}$-fluoride, PET/CT allows for localization and quantification of bone formation, bone osteolysis and bone remodeling. CT visualizes mineralized bone that has been deposited and remodeled as a result of the activity of the osteoblasts, up to the moment of scanning. ${ }^{18} \mathrm{~F}$-fluoride PET visualizes the activity of osteoblasts at the moment of scanning, and hence could be considered a precursor for the mineralization process.

In this thesis, the use of ${ }^{18} \mathrm{~F}$-fluoride PET/CT scanning in patients early after spinal fusion as well as in patients with persistent or recurrent symptoms several years after fusion surgery, was evaluated. In early postoperative patients, we hypothesized that PET activity within the operated segment could be an early predictor of the bone fusion status on CT at a later stage. In patients with persistent pain, we hypothesized that PET/CT could identify possible sources of pain by locating areas of increased bone stress and remodeling.

The specific objectives of this thesis were:

To provide an overview of the diagnostic accuracy of imaging modalities to detect pseudarthrosis after spinal fusion, with surgical exploration as reference standard

A systematic literature review was performed in Chapter 2 to identify studies that evaluated the diagnostic accuracy of imaging modalities to detect pseudarthrosis after thoracolumbar spinal fusion with surgical exploration as reference standard. Studies that evaluated the modalities plain radiography, flexion extension radiography, CT, scintigraphy, tomography, ultrasound and PET/CT were identified and included in the review. Metaanalyses with the odds ratio as outcome measure were performed to summarize the results of studies that evaluated the modalities plain radiography, flexion extension radiography, CT and scintigraphy. Based on the odds ratios obtained after meta-analyses, CT was considered the most accurate non-invasive modality for the detection of pseudarthrosis after thoracolumbar spinal fusion.

To investigate the relationship between ${ }^{18}$ F-fluoride PET/CT, CT and clinical status several years after PLIF in symptomatic patients and asymptomatic patients

From Chapter 2 we concluded that CT is the most accurate non-invasive modality to detect whether fusion has been established or whether a patient suffers from pseudarthrosis after spinal fusion.

However, as a result of the variable relationship between anatomical findings and symptoms of spinal fusion patients, the value of modalities like CT can be limited in patients 
that suffer from persistent or recurrent pain after PLIF. Symptoms of a patient are very important when deciding on a treatment; a patient without symptoms is rarely treated. In Chapter 3 and Chapter 4 spinal fusion patients were evaluated by means of ${ }^{18} \mathrm{~F}$-fluoride PET/CT and findings were correlated to patients' symptoms as measured by PROMs. Asymptomatic patients as well as symptomatic patients were included in the study cohort to learn about "normal bone metabolism values" of a spinal segment after PLIF as well as possible deviations therefrom in symptomatic patients. In Chapter 3, anatomical findings on $\mathrm{CT}$ and functional findings on ${ }^{18} \mathrm{~F}$-fluoride $\mathrm{PET} / \mathrm{CT}$ in regions within the operated segment were correlated to PROMs. In Chapter 4, CT and PET/CT findings of the facet joints, pedicle screws and adjacent segments were taken into account additionally.

In both chapters we confirmed the variable relationship between anatomical findings on $\mathrm{CT}$ and symptoms of a patient measured by PROMs. The spinal structures for which biological PET/CT findings correlated best to PROM scores were the facet joints, the lower endplate of the operated segment and the screws. In asymptomatic patients, we observed that a spinal fusion segment showed increased bone metabolism values in the adjacent facet joints and surrounding the screws to a certain extent, inherent to the surgical procedure performed. Deviating high increases in bone metabolism of facet joints, lower endplate of the operated segment and screw surroundings can point to pain generators in spinal fusion patients with persisting symptoms. Furthermore, we found an inverse correlation between fusion on $\mathrm{CT}$ and bone metabolism activity in the operated segment on PET/CT, as well as a linear correlation between the amount of subsidence on $\mathrm{CT}$ and the bone metabolism activity in the facet joints. From Chapter 3 and 4, we concluded that functional information from ${ }^{18} \mathrm{~F}$-fluoride PET/CT scans can be an important complement to the anatomical information from $\mathrm{CT}$ in the evaluation of symptomatic spinal fusion patients, and thus helpful in clinical decision-making.

To develop an analysis method for dynamic ${ }^{18} \mathrm{~F}$-fluoride PET/CT scans of patients after PLIF

In order to explore the use of dynamic ${ }^{18} \mathrm{~F}$-fluoride PET/CT scanning in patients early after PLIF in Chapter 6, a method to analyze the dynamic scans was developed in Chapter 5.

In Chapter 5 we validated an analysis approach to extract bone metabolism parameters from a 30-minute dynamic PET/CT scan with an image derived input function in the aorta as the arterial input.

To investigate the potential of a ${ }^{18}$ F-fluoride PET/CT scan early after PLIF to predict fusion outcome at one year

In Chapter 6, twenty patients undergoing PLIF were prospectively included. A diagnostic CT one year after surgery was used to distinguish between patients with fusion and patients developing pseudarthrosis. Bone metabolism values at six weeks and at one year after PLIF were compared between these groups. The most important finding was that 
six weeks after PLIF, the bone metabolic activity $\left(S U V_{\max }, K_{i}\right)$ in the operated intervertebral disc space was significantly lower in patients who were categorized as pseudarthrosis at one year as compared to patients who attained solid interbody fusion one year postoperatively. By means of full pharmacokinetic analysis, deviating patterns between pseudarthrotic and fused segments were also shown in parameters related to bone blood flow $\left(K_{1} / k_{2}\right)$ and bone mineral incorporation $\left(k_{3} /\left(k_{2}+k_{3}\right)\right)$. Pseudarthrotic segments exhibited lower bone blood flow towards the operated segment in comparison to fused segments six weeks postoperatively, which may have resulted in less bone mineral incorporation in the intervertebral disc space at one year. In short, based on the results of ${ }^{18} \mathrm{~F}$-fluoride PET/CT scans six weeks after PLIF, we were able to show differences between patients that attained fusion and patients developing pseudarthrosis at one year after PLIF. 


\section{RECOMMENDATIONS}

The course of bone metabolism values after PLIF

Diagnosis of a certain disorder by means of PET is based on an abnormal distribution of the administered tracer. To determine whether the tracer distribution in a patient is abnormal or not, knowledge on the tracer distribution in subjects without the disorder is required. Therefore, for ease of data interpretation it was important to include patients with and without symptoms in Chapter 3 and 4 and patients with and without successful fusion in Chapter 6. Besides operated segments, we also evaluated non-operated segments to evaluate bone metabolism values in the "normal" situation.

In a non-operated segment, the bone metabolic activity at the end plates was higher compared to in the intervertebral disc space. The intervertebral disc space is cartilage-like, contains no hydroxyapatite and therefore no fluoride uptake will be localized in the intervertebral disc space. Bone at the endplates undergoes bone remodeling as a result of daily pressure on the bone and therefore displays fluoride uptake.

In a PLIF segment that will successfully fuse, the bone metabolic activity at six weeks after PLIF at the endplates and in the intervertebral disc space was significantly increased to an equally high level. Also, bone blood flow to the operated segment at six weeks was increased compared to non-operated levels, which can be a result of routinely performed surgical abrasion of the endplates to enhance blood flow to the bone graft [1]. Over the course of time, successfully fused segments displayed a significant decrease in bone metabolism values from six weeks to one year after surgery. Bone metabolic activity values at one year postoperative were still increased compared to non-operated segments, which is consistent with literature showing elevated bone metabolism values within operated bone regions over a course of several years after surgery [2-5].

In a PLIF segment that will develop pseudarthrosis, a pattern of high endplate metabolism and lower activity in the intervertebral disc space at six weeks was seen on ${ }^{18} \mathrm{~F}$-fluoride $\mathrm{PET} / \mathrm{CT}$, similar to non-operated segments, however with higher absolute values. Bone blood flow to the operated segments at six weeks in these pseudarthrosis patients was significantly lower compared to the patients that attained fusion. Furthermore, bone metabolism activity in the operated segment did not decrease from six weeks to one year. This finding is in accordance with findings on impaired graft healing of Brenner et al. [2]. The sustained high bone metabolism values of the operated segment at one year might indicate that the fusion process was not completed yet. Incomplete fusion is a risk factor for instability which is likely to cause bone stress on adjacent structures that can potentially generate pain.

Spinal fusion patients can develop recurrent symptoms several months to years after surgery. In case these symptoms are related to changes in load distribution of the spinal column that are reflected in local bone remodeling osteoblastic activity, ${ }^{18} \mathrm{~F}$-fluoride 
$\mathrm{PET} / \mathrm{CT}$ can be of value in setting the diagnosis. When interpreting these scans, it is important to realize that spinal fusion itself (whether successful or not) is associated with biomechanical changes of the spinal column and therefore changes in osteoblast activity. Elevated activity to a certain extent in the facet joints, operated segment and screws were considered a normal finding in a patient after PLIF. Therefore, not every hotspot on a $\mathrm{PET} / \mathrm{CT}$ scan is necessarily related to pain symptoms. In this thesis it was shown that hotspots in the facet joints of the upper adjacent segment, the screw surroundings and the lower endplate of the operated segment were prone to be related to symptoms.

\section{Clinical applicability}

In this thesis, ${ }^{18} \mathrm{~F}$-fluoride PET/CT scans were analyzed by means of SUV calculation as well as by full pharmacokinetic modeling. In clinical practice, ${ }^{18} \mathrm{~F}$-fluoride PET/CT scanning can be used in patients with persistent or recurrent symptoms after PLIF. Static scanning and SUV calculations are sufficient for this purpose. One of the main limitations in Chapter 3 and 4 was that we could not confirm our PET/CT findings by surgical verification or by proof of successful treatment of the suspected pain generator based on the PET/CT findings. The next step would be to base treatment on these findings and follow-up the success of treatment accordingly, to determine whether the diagnosis of ${ }^{18} \mathrm{~F}$-fluoride PET/CT was correct and resulted in appropriate clinical decision making. Multiple follow-up moments including ${ }^{18} \mathrm{~F}$-fluoride PET/CT scanning would be interesting to evaluate the changes in bone metabolism patterns over time in relation to the changes in the clinical symptoms of a patient.

In clinical practice, patients with symptoms after PLIF can also be evaluated by means of SPECT/CT [6-11]. This technique uses technetium-99m labeled phosphonate molecules as tracer which is absorbed onto or into the crystalline structure of hydroxyapatite to mark bone remodeling, similar to the uptake mechanism of ${ }^{18} \mathrm{~F}$-fluoride [12]. Imaging with technetium-99m labeled phosphonate molecules employs a conventional nuclear gamma camera, which is cheaper and more widely available compared to PET/CT, but suffers from low image resolution in detailed bone and has limited quantification capabilities, which is a disadvantage when monitoring bone formation over time in follow-up. However, in clinical follow-up these disadvantages might not be relevant and moreover, recent studies highlight advancements in quantification in SPECT/CT [13].

By means of full pharmacokinetic modeling, we were able to show differences between fusion and pseudarthrosis patients at six weeks after surgery in a prospective patient group, regardless of the relatively small patient group of twenty. However, the applicability of this information in clinical practice is limited as yet. We did not perform longterm follow-up of these patients to evaluate whether the patients diagnosed with pseudarthrosis on CT at one year after surgery also developed clinical symptoms later on. Therefore, at this stage, it is not recommendable to scan each patient that underwent 
PLIF at six weeks after surgery by means of ${ }^{18} \mathrm{~F}$-fluoride PET/CT and take action when the bone metabolism pattern resembles that of a pseudarthrosis patient. However, the ability of pharmacokinetic modeling after ${ }^{18} \mathrm{~F}$-fluoride PET/CT to evaluate bone blood flow separately from general bone metabolism, provides insights into the spinal fusion process and offers research opportunities, especially in the early postoperative phase.

In Chapter 6, one early time point ${ }^{18} \mathrm{~F}$-fluoride PET/CT scan was acquired. The six weeks timepoint was arbitrarily chosen in analogy to the process of fracture healing in which the first signs of healing can be expected within six weeks. We are aware of the fact that fusion of vertebrae is a different process from fracture healing and six weeks might not be the most optimal time point to extract prognostic information. In order to find out which time point after spinal fusion would provide the most valuable prognostic information, multiple ${ }^{18} \mathrm{~F}$-fluoride PET/CT scans would have to be made early after surgery and compared to later outcome.

\section{FUTURE CONSIDERATIONS}

Long-term follow-up prospective patients

In Chapter 6 we have shown that ${ }^{18} \mathrm{~F}$-fluoride PET/CT at six weeks showed different bone metabolism patterns for patients developing pseudarthrosis compared to patients attaining fusion as assessed at one year after surgery on $\mathrm{CT}$, in twenty prospectively included patients. The main limitation of this study was that the differentiation between fusion and pseudarthrosis was based on a diagnostic CT scan at one year after surgery, while bony fusion and mineralization of the fusion mass might occur later than one year postoperatively. This limitation would be solved by the addition of a long-term follow-up time point (more than five years after surgery) for the twenty patients, including a diagnostic CT scan. The CT scan could be used to further differentiate between delayed fusion and definite pseudarthrosis. Subsequently, we could evaluate whether the results of six weeks PET/CT also correlate to the long-term fusion assessment. At that same long-term visit, we could ask the patients to fill out questionnaires (ODI, VAS, EQ-5D) to determine whether the patients that were diagnosed with pseudarthrosis on long-term CT, also developed recurrent symptoms. Ideally, the long-term visit would also include another ${ }^{18} \mathrm{~F}$ fluoride PET/CT scan. We could evaluate whether the bone metabolism values in the operated segment in the patients that attained fusion, have further decreased down to bone metabolism values found in non-operated segments. We could evaluate what happened with the sustained high bone metabolism values in the patients that did not attain fusion at the one year time point. Moreover, we could apply the analysis techniques proposed in Chapters 3 and 4 to see whether the patients that developed recurrent symptoms as assessed by the long-term questionnaires, also show hotspots at the facet joints, 
endplates or screw surroundings on the long-term PET/CT image. The addition of a longterm visit would make the results of Chapter 6 more conclusive and would nicely bring together the research performed in Chapter 3 and 4 with the research performed in Chapter 6.

\section{Alternative bone grafts}

The patients described in Chapter 3-6 underwent PLIF surgery in which autologous bone graft from the lamina was used to fill the PEEK cages before insertion into the disc space. In some cases, the quantity of available autologous bone may be limited and/or the autologous bone is expected to be of insufficient quality. As a result, numerous alternatives for autologous bone graft have been investigated. A bone grafting material should provide: osteoinductive factors which allow for the osteoprogenitor cells to differentiate into osteoblasts; osteogenic cells which directly lay down new bone matrix; and an osteoinductive matrix onto which bone matrix can be deposited [14]. Potential alternatives to locally harvested autograft include iliac crest autograft, human allograft, calcium-phosphate salts, demineralized bone matrix, bone morphogenic proteins.

At present, a clinical trial is performed in our department in which a biomaterial is used to fill the PEEK cages before insertion into the disc space, instead of autologous bone graft. Follow-up of these patients includes ${ }^{18} \mathrm{~F}$-fluoride PET/CT scanning at six weeks and at one year similar to the study protocol in Chapter 6. The findings in these patients can directly be compared to the autologous group of Chapter 6 to evaluate the efficacy of the graft material. Besides alternative bone grafts, the effect of alternative cage designs could be tested by means of ${ }^{18} \mathrm{~F}$-fluoride PET/CT as well.

Multiple ${ }^{18}$ F-fluoride PET/CT follow-up time points in a preclinical sheep model for spinal fusion

One of the limitations in the prospective study of Chapter 6 was that ${ }^{18} \mathrm{~F}$-fluoride PET/CT scanning was performed at one early time point only, at six weeks postoperatively. Ideally, multiple ${ }^{18} \mathrm{~F}$-fluoride PET/CT measurements per patient would be acquired after spinal fusion surgery. This would allow follow-up of the bone formation and the bone remodeling processes, and would help to determine the optimal time point for collection of prognostic information. However, the concomitant radiation exposure that would be implied is not ethical nor justifiable in patients without direct benefit. Besides the radiation exposure, this type of research would mean a huge time burden for the patient and involve high costs and is therefore not feasible in humans. As an alternative, we have designed a preclinical study in which sheep are subjected to spinal fusion surgery with 
follow-up by means of multiple ${ }^{18}$ F-fluoride PET/CT scans afterwards. Sheep are considered a good model for spinal fusion and have been frequently used in that context [1522].

A pilot study of seven sheep has been performed to develop a model for spinal fusion in sheep with follow-up by means of ${ }^{18} \mathrm{~F}$-fluoride PET/CT scanning at three, six, nine and 26 weeks after surgery. Spinal fusion was performed by a retroperitoneal approach, as described by Baramki et al. [23]. The intervertebral disc of the segment $L 4-L 5$ was excised, and the endplates were thoroughly decorticated. Autologous bone from the ipsilateral iliac crest was harvested and used to fill a custom-made PEEK cage which was subsequently implanted in the excised discus space between L4 and L5. For the PET/CT scanning procedure, the sheep were anesthetized and positioned on a clinical integrated PET/CT scanner (Gemini TF 64 PET-CT, Philips, the Netherlands) (Figure 1A). Intravenous injection of ${ }^{18} \mathrm{~F}$-fluoride into the front leg of the sheep was directly followed by dynamic scanning for 30 minutes. Also, a diagnostic CT scan was acquired. In Figure $1 B$ a PET image just after injection of the tracer is presented. Figure $1 \mathrm{C}$ shows a PET image at 30 minutes after injection of the tracer, merged with the CT image. The tracer has been deposited in the bone and in higher extent deposited at the site of surgery. Pharmacokinetic modeling with a ROI in the aorta as image derived input function was performed as suggested in Chapter 5 and 6 to obtain the parameters $K_{i}, K_{1} / k_{2}$ and $k_{3} /\left(k_{2}+k_{3}\right)$ at the different time points after surgery. Figure 2 shows the bone metabolism values ( $\mathrm{Ki}$ ) of the endplates and intervertebral disc space in the operated segment as well as in the control segment over time. At three weeks, the bone metabolism activity in the control regions and in the operated regions were fairly similar, with a high standard deviation between the sheep. Therefore, the values at the three weeks time point seem to be clouded by surgical effects and not of value. The activity pattern of the six regions looked similar at six and nine weeks and show separation of the operated and control segments. At 26 weeks, the bone metabolism values of the operated regions have decreased to a level similar to the control regions. 

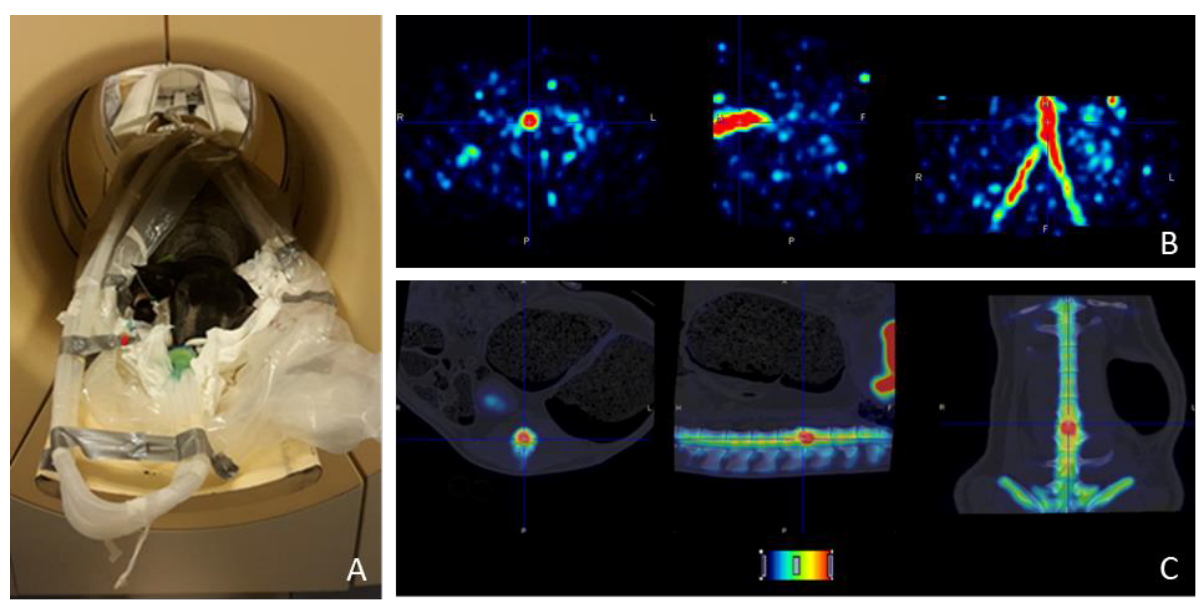

Figure 1. (A) positioning of an anesthetized and ventilated sheep on the PET/CT bed. A plastic sheet was used to preclude spilling of potential urine and/or saliva. Ventilation tubes were fixated to the PET/CT bed. (B) an axial, sagittal and coronal PET image early after injection of ${ }^{18} \mathrm{~F}$-fluoride showing the activity in the aorta. (C) an axial, sagittal and coronal fused PET/CT image of the lower spine after spinal fusion.

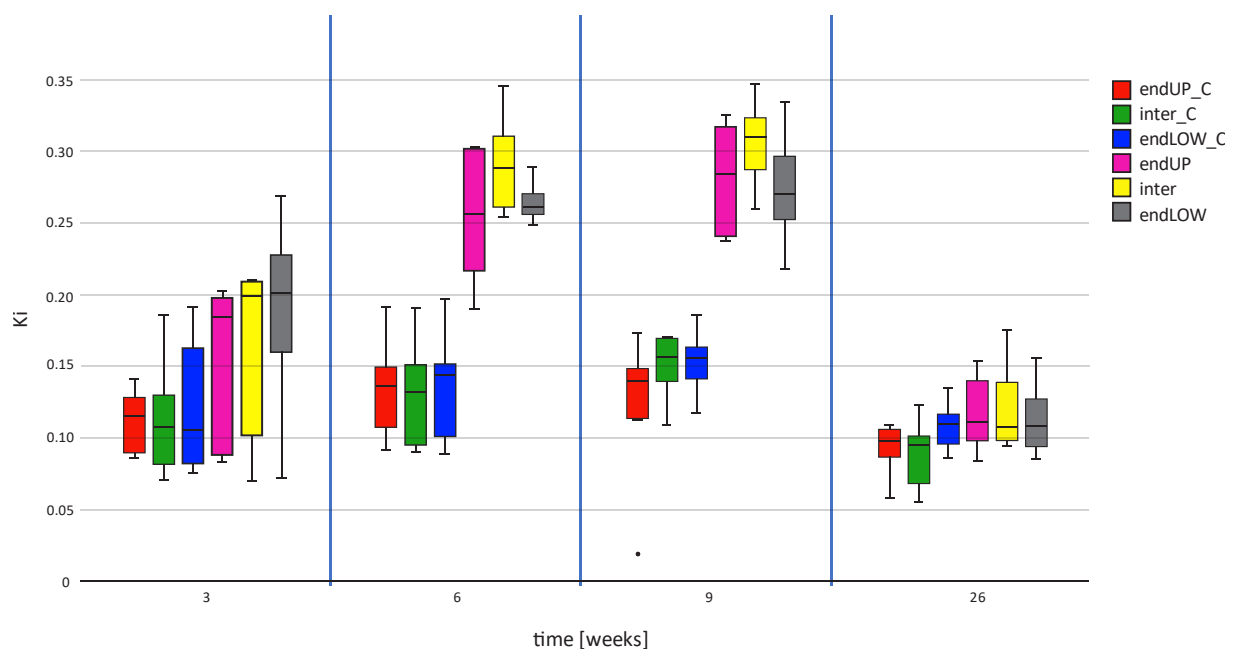

Figure 2. $K_{i}$ of the control ROls (endUP_C, inter_C, endLOW_C in red, green and blue respectively) and the operated ROIs (endUP, inter, endLOW in pink, yellow and grey respectively) for the seven sheep at 3 weeks, 6 weeks, 9 weeks and 26 weeks after spinal fusion surgery. 
Besides in vivo follow-up, preclinical studies also allow for post-mortem tests that are not possible to perform in human research. Post-mortem tests that could be considered for this type of research include biomechanical testing, micro-computed tomography (microCT) and histology. Biomechanical testing can be performed to determine the range of motion within a fusion construct as compared to a non-operated (adjacent) segment. Cyclic pure moments can be applied to the vertebral column in flexion and extension, lateral bending and axial rotation. The resulting motion of the individual vertebrae can be tracked by marker-based tracking systems. The quality of the bony bridges formed between vertebrae can be assessed by means of microCT (example given in Figure 3, left). High-resolution images of the cage space can be produced from which several parameters such as bone volume as a fraction of the total cage volume, the trabecular number or the average trabecular thickness can be calculated. Histology can be used to evaluate the vertebral segments microscopically. Specific stainings can be used to represent different types of tissues by different colors. Specifically, the Masson Goldner Trichrome staining (example given in Figure 3, right) can be used to make a distinction between new bone matrix or osteoid (red/orange) and mature bone matrix (green) [24]. Osteoblasts and osteoclasts are also visualized [24]. Also, different fluorochrome labels can be administered to follow the mineralization fronts per time point with fluorescence microscopy post-mortem. Fluorochromes are calcium-seeking substances that are absorbed into new mineralized bone tissue and can therefore be used to observe bone formation and bone remodeling over time. Fluorochromes contain fluorophores; molecules with fluorescent properties. These properties allow visualization of the mineralization surfaces under a light- or fluorescence microscope using ultraviolet or blue light [25].

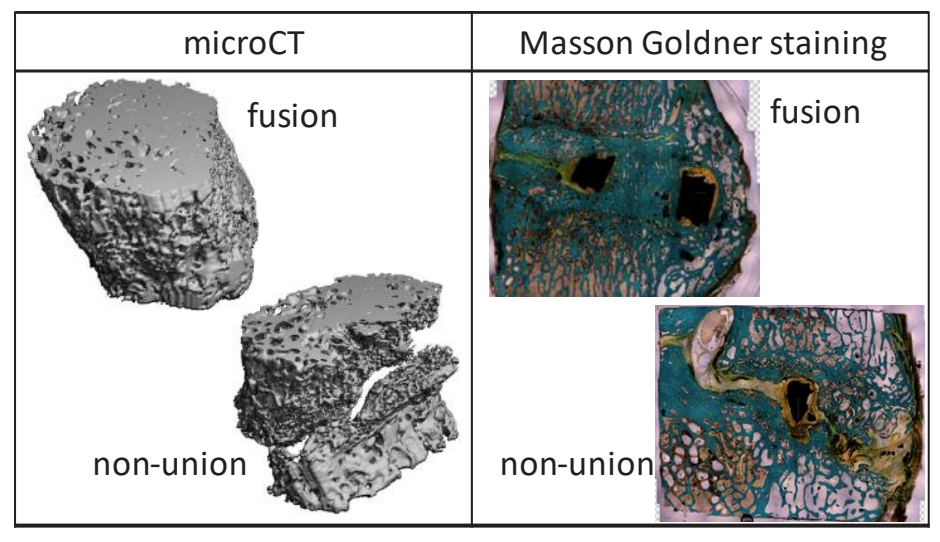

Figure 3. Examples of post-mortem test options in a sheep spinal fusion model. (left) MicroCT images of the fusion mass inside a spinal fusion cage, showing an example of a complete fusion and a non-union (pseudarthrosis). (right) Sagittal slices (through the cage, depicted in black) of a segment after spinal fusion, stained by Masson-Goldner to differentiate between different types of tissues between the vertebrae. The two examples show a slice of a fused segment with mature bone matrix within the cage, shown in green, and a slice of a nonunion segment lacking the solid green mass within the cage. 
The model that was developed for spinal fusion in sheep, including follow-up by means of ${ }^{18} \mathrm{~F}$-fluoride PET/CT, can be used to evaluate new cage designs, as well as new grafting materials in a research setting. The combination of ${ }^{18} \mathrm{~F}$-fluoride PET/CT results with postmortem tests, could also help to deepen the understanding and interpretation of ${ }^{18} \mathrm{~F}$ fluoride PET/CT scanning, which can be translated to the human situation.

\section{Other applications of ${ }^{18}$ F-fluoride PET/CT in orthopedics}

${ }^{18} \mathrm{~F}$-fluoride PET/CT may also be applied to other orthopedic conditions that involve changes of bone metabolism values. For example, follow-up of patients after fracture healing $[26,27]$ or osteotomy [28]. Also, ${ }^{18}$ F-fluoride PET/CT can be used to follow-up the course of several metabolic diseases such as rheumatoid arthritis [29], Paget disease [30], osteoporosis [31] and osteoarthritis [32,33], or to distinguish between septic and aseptic loosening of hip prostheses [34] or detect loosening of knee prostheses [35]. Within our department, ${ }^{18} \mathrm{~F}$-fluoride PET/CT was explored as a follow-up measure of bone metabolism activity after total hip arthroplasty (THA) as one other application. Newly designed implants need safe introduction with high-quality monitoring. Several imaging modalities are being used to assess whether failure of a prosthesis has occurred, such as routine radiography, $\mathrm{CT}$, DEXA, MRI, subtraction arthrography, nuclear arthrography, or bone scintigraphy. Radiostereometric analysis (RSA) can be used to assess early prosthesis migration, which has been shown to be a predictor of aseptic loosening $[36,37]$. To add a biological component to the means of follow-up, we explored the potential of ${ }^{18} \mathrm{~F}$-fluoride $\mathrm{PET} / \mathrm{CT}$ scanning to evaluate bony ingrowth into cup designs after THA. To be able to do so, ${ }^{18} \mathrm{~F}$-fluoride PET/CT scanning was performed at 13 weeks, 26 weeks and one year after THA in five pilot patients. Figure 4 shows a ${ }^{18} \mathrm{~F}$-fluoride PET image, a CT image and a combination of the two in a patient at 13 weeks after THA. 

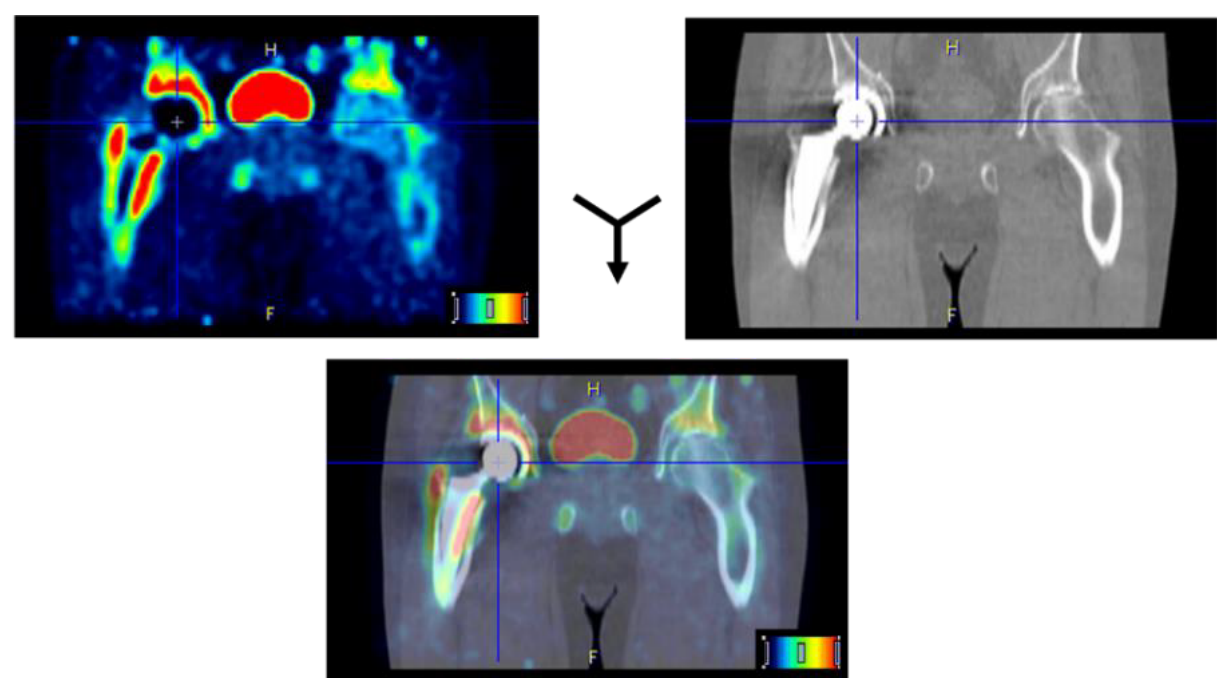

Figure 4. PET image and CT image of a patient at 13 weeks after THA of the right hip (left side on the image) that can be fused to allow for localization of bone metabolism.

A custom-made routine was written to visualize bone metabolism values around the cup in three dimensions and to quantify the values in two dimensions (Figure 5). The routine can also be applied to visualize bone metabolism values surrounding the stem of a hip prosthesis (Figure 6). This technique facilitates analysis of bone metabolism values around hip prostheses and allows comparisons of ${ }^{18} \mathrm{~F}$-fluoride PET/CT measurements over time. This semi-automatic method to evaluate ${ }^{18} \mathrm{~F}$-fluoride PET/CT scans of patients after THA is promising and can be used in larger cohort studies comparing different prosthesis designs. Validation studies including long-term outcome should be performed to assess the potential of the technique in the early evaluation of THA prostheses.
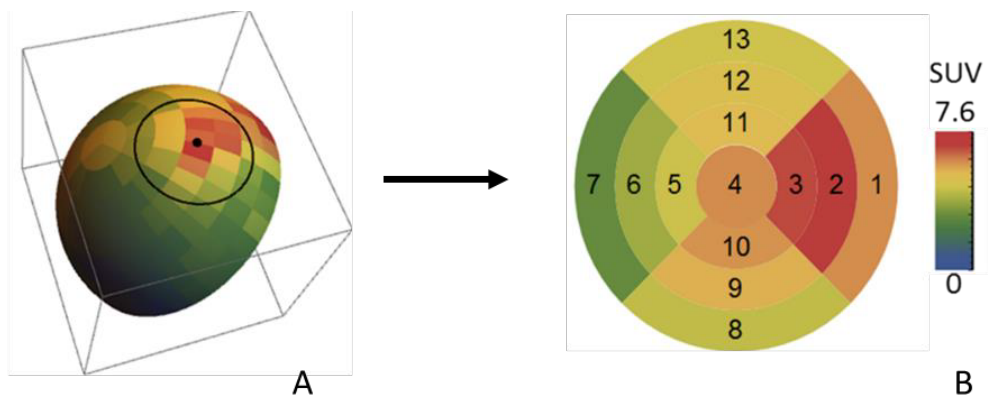

Figure 5. The custom-written analysis method results in a three-dimensional visualization of bone metabolism values around the cup, with the superolateral region indicated by a dot and surrounding circle (A). For interpretation and quantification purposes, values were also displayed as SUV values in a two-dimensional map with self-defined numbered segments to simplify follow-up of bone metabolism surrounding the cup over time (B). 


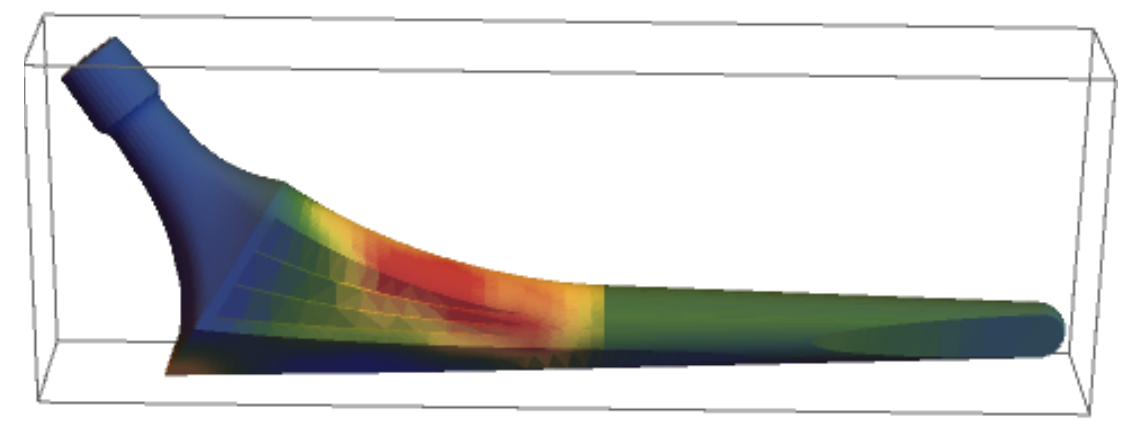

Figure 6. An example of a three-dimensional visualization of bone metabolism values around the stem when the analysis method is subjected to a stem in a patient after THA surgery.

\section{FINAL CONCLUSION}

${ }^{18} \mathrm{~F}$-fluoride PET/CT can be a useful addition in the follow-up of patients after lumbar spinal interbody fusion. In clinical practice, hotspots indicating possible pain generators can be detected in patients with persistent or recurrent symptoms by means of static ${ }^{18} \mathrm{~F}$ fluoride PET/CT scanning. In a clinical or preclinical research setting, pharmacokinetic modeling can be applied in an early stage after spinal fusion to predict the fusion result at one year after surgery.

The findings in this thesis could be further explored by the evaluation spinal fusion patients with other types of bone graft or cage designs. The application of ${ }^{18} \mathrm{~F}$-fluoride $\mathrm{PET} / \mathrm{CT}$ in the orthopedic field could be expanded to patients undergoing total hip arthroplasty to assess the activity of bone metabolism surrounding a prosthesis as an indicator of bone ingrowth. 


\section{REFERENCES}

1. Herkowitz HN, Garfin SR, Eismont FJ, Bell GR, Balderston RA. Rothman-Simeone The Spine E-Book: Expert Consult: Elsevier Health Sciences, 2011.

2. Brenner W, Vernon C, Conrad EU, Eary JF. Assessment of the metabolic activity of bone grafts with (18)Ffluoride PET. European journal of nuclear medicine and molecular imaging. 2004; 31(9):1291-1298.

3. Heekin RD, Engh CA, Vinh T. Morselized allograft in acetabular reconstruction. A postmortem retrieval analysis. Clinical orthopaedics and related research. 1995(319):184-190.

4. Hooten JP, Jr., Engh CA, Heekin RD, Vinh TN. Structural bulk allografts in acetabular reconstruction. Analysis of two grafts retrieved at post-mortem. The Journal of bone and joint surgery British volume. 1996; 78(2):270-275.

5. Piert M, Winter E, Becker GA, Bilger K, Machulla H, Muller-Schauenburg W, et al. Allogenic bone graft viability after hip revision arthroplasty assessed by dynamic [18F]fluoride ion positron emission tomography. European journal of nuclear medicine. 1999; 26(6):615-624.

6. Sumer J, Schmidt D, Ritt P, Lell M, Forst R, Kuwert T, et al. SPECT/CT in patients with lower back pain after lumbar fusion surgery. Nuclear medicine communications. 2013; 34(10):964-970.

7. Hudyana H, Maes A, Vandenberghe T, Fidlers L, Sathekge M, Nicolai D, et al. Accuracy of bone SPECT/CT for identifying hardware loosening in patients who underwent lumbar fusion with pedicle screws. European journal of nuclear medicine and molecular imaging. 2016; 43(2):349-354.

8. Freiermuth D, Kretzschmar M, Bilecen D, Schaeren S, Jacob AL, Aeschbach A, et al. Correlation of (99m) TC-DPD SPECT/CT Scan Findings and Diagnostic Blockades of Lumbar Medial Branches in Patients with Unspecific Low Back Pain in a Randomized-Controlled Trial. Pain medicine (Malden, Mass). 2015; 16(10):1916-1922.

9. Lopez Rodriguez E, Garcia Jimenez R, Sanchez Aguilar M, Valencia Anguita J, Luis Simon J. Hybrid imaging (SPECT/computed tomography) with three-dimensional reconstruction: a new diagnostic tool for the study of low back pain after spinal fusion surgery. The spine journal : official journal of the North American Spine Society. 2015; 15(2):377-378.

10. Littenberg B, Siegel A, Tosteson AN, Mead T. Clinical efficacy of SPECT bone imaging for low back pain. Journal of nuclear medicine : official publication, Society of Nuclear Medicine. 1995; 36(9):1707-1713.

11. Rager O, Schaller K, Payer M, Tchernin D, Ratib O, Tessitore E. SPECT/CT in differentiation of pseudarthrosis from other causes of back pain in lumbar spinal fusion: report on 10 consecutive cases. Clinical nuclear medicine. 2012; 37(4):339-343.

12. Wong KK, Piert M. Dynamic bone imaging with 99mTc-labeled diphosphonates and 18F-NaF: mechanisms and applications. Journal of nuclear medicine : official publication, Society of Nuclear Medicine. 2013; 54(4):590-599.

13. Dickson J, Ross J, Voo S. Quantitative SPECT: the time is now. EJNMMI physics. 2019; 6(1):4.

14. Ludwig SC, Kowalski JM, Boden SD. Osteoinductive bone graft substitutes. European spine journal : official publication of the European Spine Society, the European Spinal Deformity Society, and the European Section of the Cervical Spine Research Society. 2000; 9 Suppl 1:S119-125.

15. Assad M, Jarzem P, Leroux MA, Coillard C, Chernyshov AV, Charette S, et al. Porous titanium-nickel for intervertebral fusion in a sheep model: part 1. Histomorphometric and radiological analysis. Journal of biomedical materials research Part B, Applied biomaterials. 2003; 64(2):107-120. 
16. Brodano GB, Giavaresi G, Lolli F, Salamanna F, Parrilli A, Martini L, et al. Hydroxyapatite-Based Biomaterials Versus Autologous Bone Graft in Spinal Fusion: An In Vivo Animal Study. Spine. 2014; 39(11):E661-E668.

17. Fredericks DC, Gandhi AA, Grosland NM, Smucker JD. Assessment of BioPlex interbody fusion device in a sheep lumbar fusion model. The lowa orthopaedic journal. 2013; 33:33-39.

18. Humadi A, Freeman BJ, Moore RJ, Callary S, Halldin K, David V, et al. A comparison of radiostereometric analysis and computed tomography for the assessment of lumbar spinal fusion in a sheep model. Evidence-based spine-care journal. 2013; 4(2):78-89.

19. Magin MN, Delling G. Improved lumbar vertebral interbody fusion using rhOP-1: a comparison of autogenous bone graft, bovine hydroxylapatite (Bio-Oss), and BMP-7 (rhOP-1) in sheep. Spine. 2001; 26(5):469-478.

20. Solchaga LA, Hee CK, Aguiar DJ, Ratliff J, Turner AS, Seim HB, 3rd, et al. Augment bone graft products compare favorably with autologous bone graft in an ovine model of lumbar interbody spine fusion. Spine. 2012; 37(8):E461-467.

21. Sugiyama S, Wullschleger M, Wilson K, Williams R, Goss B. Reliability of clinical measurement for assessing spinal fusion: an experimental sheep study. Spine. 2012; 37(9):763-768.

22. Yamada K, Ito M, Akazawa T, Murata M, Yamamoto T, Iwasaki N. A preclinical large animal study on a novel intervertebral fusion cage covered with high porosity titanium sheets with a triple pore structure used for spinal fusion. European spine journal : official publication of the European Spine Society, the European Spinal Deformity Society, and the European Section of the Cervical Spine Research Society. 2015; 24(11):2530-2537.

23. Baramki HG, Papin P, Steffen T. A surgical approach to the ventral aspect of the lumbar vertebrae in the sheep model. Surgical and radiologic anatomy : SRA. 2000; 22(1):25-27.

24. Goldschlager T, Abdelkader A, Kerr J, Boundy I, Jenkin G. Undecalcified bone preparation for histology, histomorphometry and fluorochrome analysis. Journal of visualized experiments : JoVE. 2010(35).

25. Erben RG. Bone-labeling techniques. Handbook of histology methods for bone and cartilage 2003:pp. 99117.

26. Hsu WK, Feeley BT, Krenek L, Stout DB, Chatziioannou AF, Lieberman JR. The use of 18F-fluoride and 18FFDG PET scans to assess fracture healing in a rat femur model. European journal of nuclear medicine and molecular imaging. 2007; 34(8):1291-1301.

27. Lundblad H, Karlsson-Thur C, Maguire GQ, Jr., Jonsson C, Noz ME, Zeleznik MP, et al. Can Spatiotemporal Fluoride ((18)F(-)) Uptake be Used to Assess Bone Formation in the Tibia? A Longitudinal Study Using PET/CT. Clinical orthopaedics and related research. 2017; 475(5):1486-1498.

28. Mechlenburg I, Hermansen F, Thillemann T, Soballe K. Blood perfusion and bone formation before and after minimally invasive periacetabular osteotomy analysed by Positron Emission Tomography combined with Computed Tomography. International orthopaedics. 2013; 37(5):789-794.

29. Watanabe T, Takase-Minegishi K, Ihata A, Kunishita Y, Kishimoto D, Kamiyama R, et al. (18)F-FDG and (18)F$\mathrm{NaF} \mathrm{PET} / \mathrm{CT}$ demonstrate coupling of inflammation and accelerated bone turnover in rheumatoid arthritis. Modern rheumatology. 2016; 26(2):180-187.

30. Installe J, Nzeusseu A, Bol A, Depresseux G, Devogelaer JP, Lonneux M. (18)F-fluoride PET for monitoring therapeutic response in Paget's disease of bone. Journal of nuclear medicine : official publication, Society of Nuclear Medicine. 2005; 46(10):1650-1658.

31. Frost ML, Blake GM, Fogelman I. (18)F-Fluoride PET in Osteoporosis. PET clinics. 2010; 5(3):259-274. 
32. Kobayashi N, Inaba Y, Tateishi U, Ike H, Kubota S, Inoue T, et al. Comparison of 18F-fluoride positron emission tomography and magnetic resonance imaging in evaluating early-stage osteoarthritis of the hip. Nuclear medicine communications. 2015; 36(1):84-89.

33. Kobayashi N, Inaba Y, Tateishi U, Yukizawa Y, Ike H, Inoue T, et al. New application of $18 F-$ fluoride PET for the detection of bone remodeling in early-stage osteoarthritis of the hip. Clinical nuclear medicine. 2013; 38(10):e379-383.

34. Adesanya O, Sprowson A, Masters J, Hutchinson C. Review of the role of dynamic 18F-NaF PET in diagnosing and distinguishing between septic and aseptic loosening in hip prosthesis. Journal of orthopaedic surgery and research. 2015; 10:5.

35. Sterner T, Pink R, Freudenberg L, Jentzen T, Quitmann H, Bockisch A, et al. The role of [18F]fluoride positron emission tomography in the early detection of aseptic loosening of total knee arthroplasty. International journal of surgery (London, England). 2007; 5(2):99-104.

36. Nieuwenhuijse MJ, Valstar ER, Kaptein BL, Nelissen RG. Good diagnostic performance of early migration as a predictor of late aseptic loosening of acetabular cups: results from ten years of follow-up with Roentgen stereophotogrammetric analysis (RSA). The Journal of bone and joint surgery American volume. 2012; 94(10):874-880.

37. Pijls BG, Nieuwenhuijse MJ, Fiocco M, Plevier JW, Middeldorp S, Nelissen RG, et al. Early proximal migration of cups is associated with late revision in THA: a systematic review and meta-analysis of 26 RSA studies and 49 survivalstudies. Acta orthopaedica. 2012; 83(6):583-591. 



\section{APPENDIX}

Valorization Summary Nederlandse Samenvatting List of Publications Curriculum Vitae Dankwoord 



\section{Valorization}

Low back pain is a major global health and economic problem [1,2], with a one-year prevalence ranging from 22 to $65 \%$ and life-time prevalence of up to $84 \%$ [3]. It is the leading cause of activity limitation and work absence $[4,5]$, as well as a huge economic burden. Costs associated with low back pain include direct costs for health-care and indirect costs because of work absenteeism [6]. The total societal cost of low back pain in the Netherlands is 4.88 billion USD of which $12 \%$ accounts for direct medical costs and $88 \%$ for indirect societal costs [7]. Total cost per person in the Netherlands is 300 USD of which 36 USD is direct cost and 264 USD is indirect cost [7]. The total societal cost in the USA is 81.24 billion USD of which $47 \%$ accounts for direct medical costs and $53 \%$ accounts for indirect societal costs $[8,9]$. Total cost per person in the USA is 308 USD direct costs and 145 USD indirect costs $[8,9]$. The problem of low back pain is expected to continue to grow as a result of the aging and increasing population worldwide [5]. When conservative measures to treat low back pain, such as intensive exercise therapy, pain medication or brace immobilization fail, operative intervention can be considered. Spinal fusion is a surgical procedure in which fixation of vertebral segments is achieved by means of instrumentation and bone grafting to treat patients suffering from back pain. Pain relief is achieved by restoration of the height of the segments, decompression of the nerve roots and definite bony fusion of the vertebrae involved. Unfortunately, 5 to $50 \%$ of patients will suffer from persistent or recurrent back and/or leg pain after spinal surgery [10]. Pseudarthrosis or non-union is thought to be the cause of pain in a significant number of spinal fusion patients with persisting or recurrent symptoms [11, 12]. However, pseudarthrosis can also be asymptomatic [12-14]. In that sense, the value of imaging modalities that allow for detection of pseudarthrosis such as CT and radiography, can be limited in spinal fusion patients with complicated pain symptoms.

In this thesis, the use of ${ }^{18} \mathrm{~F}$-fluoride PET/CT scanning in patients early after spinal fusion as well as in patients with persistent or recurrent symptoms several years after fusion surgery, was evaluated. The most important findings were that static ${ }^{18} \mathrm{~F}$-fluoride PET/CT findings in patients with persistent or recurrent pain after spinal fusion, correlated better with symptoms than CT findings do, and that as early as six weeks after surgery, differences in bone metabolism can be detected between patients developing pseudarthrosis and fusion at one year after spinal fusion surgery by means of dynamic ${ }^{18} \mathrm{~F}$-fluoride PET/CT scanning. 


\section{Application of ${ }^{18} \mathrm{~F}$-fluoride PET/CT in clinical practice}

In the studies in this thesis, ${ }^{18} \mathrm{~F}$-fluoride PET/CT was not used for clinical decision making yet. In follow-up studies, we would like to base treatment after spinal fusion on PET/CT findings. For example, hotspots at facet joints could indicate a facet joint block and severe hotspots at the endplates could be an indication for revision surgery. When subsequently improved clinical follow-up results or even confirmation of PET/CT findings by observation of abnormalities during revision surgery can be shown, ${ }^{18} \mathrm{~F}$-fluoride PET/CT could be advocated to be incorporated in clinical practice of patients with persistent or recurrent pain after spinal fusion. This would potentially lead to more efficient treatment plans at an earlier time point, resulting in less uncertainty and shorter periods of limitation in daily life activities for the patient. At present, ${ }^{18} \mathrm{~F}$-fluoride PET/CT is not yet available in all hospitals. Less advanced nuclear techniques such as a technetium bone scan including SPECT/CT, are widely available and may be used as surrogate parameters [15]. Given the closer correlation with symptomatology, a SPECT/CT or PET/CT scan may be advocated as a routine procedure in patients with persisting symptoms after spinal fusion.

Economically, better diagnoses of patients with pain after spinal fusion amounting to more efficient treatment plans would decrease the direct costs for health-care as well as the indirect costs as a result of work absenteeism.

\section{${ }^{18}$ F-fluoride PET/CT as a diagnostic research tool}

By means of dynamic ${ }^{18} \mathrm{~F}$-fluoride PET/CT, parameters related to bone blood flow and bone incorporation can be calculated which can give prognostic information in an early phase after spinal fusion. The direct clinical value of this information is limited as of yet, but the technique can be used in a (pre)clinical research setting. By means of dynamic ${ }^{18}$ F-fluoride PET/CT scanning, equality or superiority of alternative graft materials to autologous bone grafts in the early postoperative phase can be demonstrated in clinical research studies. Besides alternative bone grafts, new cage designs or screws can also be evaluated. The PET/CT imaging technique can also be used as a diagnostic tool in preclinical studies. Preclinical models give the liberty to make more standardized comparisons and allow for confirmation of findings by means of histological analysis.

Besides multiple research designs that can be thought of regarding spinal fusion, the ${ }^{18} \mathrm{~F}$ fluoride PET/CT technique can also be applied to other areas where changes in bone metabolism are of value. For example, different types of fracture healing or osteotomy can be evaluated. Furthermore, several metabolic diseases such as rheumatoid arthritis, Paget's disease, osteoporosis and osteoarthritis could be studied. ${ }^{18}$ F-fluoride PET/CT could also be used to distinguish between septic and aseptic loosening of different types of prostheses, for example hip prostheses [16] or knee prostheses [17]. Prostheses designs 
nowadays are focusing more and more on early ingrowth, which is a challenge to demonstrate with common imaging modalities. Research with ${ }^{18} \mathrm{~F}$-fluoride PET/CT and its clinical application for multiple conditions can go hand in hand and consolidate each other. 


\section{REFERENCES}

1. Lambeek LC, van Tulder MW, Swinkels IC, Koppes LL, Anema JR, van Mechelen W. The trend in total cost of back pain in The Netherlands in the period 2002 to 2007. Spine. 2011; 36(13):1050-1058.

2. Volinn E. The epidemiology of low back pain in the rest of the world. Spine (Phila Pa 1976). 1997; 22(15):1798.

3. Walker BF. The prevalence of low back pain: a systematic review of the literature from 1966 to 1998. Journal of spinal disorders. 2000; 13(3):205-217.

4. Global, regional, and national incidence, prevalence, and years lived with disability for 301 acute and chronic diseases and injuries in 188 countries, 1990-2013: a systematic analysis for the Global Burden of Disease Study 2013. Lancet (London, England). 2015; 386(9995):743-800.

5. Hoy D, March L, Brooks P, Blyth F, Woolf A, Bain C, et al. The global burden of low back pain: estimates from the Global Burden of Disease 2010 study. Annals of the rheumatic diseases. 2014; 73(6):968-974.

6. Hartvigsen J, Hancock MJ, Kongsted A, Louw Q, Ferreira ML, Genevay S, et al. What low back pain is and why we need to pay attention. Lancet (London, England). 2018; 391(10137):2356-2367.

7. Lambeek LC, van Tulder MW, Swinkels IC, Koppes LL, Anema JR, van Mechelen W. The trend in total cost of back pain in The Netherlands in the period 2002 to 2007. Spine. 2011; 36(13):1050-1058.

8. Luo X, Pietrobon R, Sun SX, Liu GG, Hey L. Estimates and patterns of direct health care expenditures among individuals with back pain in the United States. Spine. 2004; 29(1):79-86.

9. Rizzo JA, Abbott TA, 3rd, Berger ML. The labor productivity effects of chronic backache in the United States. Medical care. 1998; 36(10):1471-1488.

10. Taylor RS, Taylor RJ. The economic impact of failed back surgery syndrome. British journal of pain. 2012; 6(4):174-181.

11. O'Beirne J, O'Neill D, Gallagher J, Williams DH. Spinal fusion for back pain: a clinical and radiological review. Journal of spinal disorders. 1992; 5(1):32-38.

12. Raizman NM, O'Brien JR, Poehling-Monaghan KL, Yu WD. Pseudarthrosis of the spine. The Journal of the American Academy of Orthopaedic Surgeons. 2009; 17(8):494-503.

13. Heggeness MH, Esses SI. Classification of pseudarthroses of the lumbar spine. Spine. 1991; 16(8 Suppl):S449-454.

14. Rothman RH, Booth R. Failures of spinal fusion. The Orthopedic clinics of North America. 1975; 6(1):299304.

15. Hudyana H, Maes A, Vandenberghe T, Fidlers L, Sathekge M, Nicolai D, et al. Accuracy of bone SPECT/CT for identifying hardware loosening in patients who underwent lumbar fusion with pedicle screws. European journal of nuclear medicine and molecular imaging. 2016; 43(2):349-354.

16. Adesanya O, Sprowson A, Masters J, Hutchinson C. Review of the role of dynamic 18F-NaF PET in diagnosing and distinguishing between septic and aseptic loosening in hip prosthesis. Journal of orthopaedic surgery and research. 2015; 10:5.

17. Sterner T, Pink R, Freudenberg L, Jentzen T, Quitmann H, Bockisch A, et al. The role of [18F]fluoride positron emission tomography in the early detection of aseptic loosening of total knee arthroplasty. International journal of surgery (London, England). 2007; 5(2):99-104. 


\section{Summary}

Low back pain is a major problem globally. It is the leading cause of activity limitation and work absence, as well as a huge economic burden. Costs associated with low back pain include direct costs for health-care and indirect costs as a result of work absenteeism. The problem of low back pain is expected to continue to grow as a consequence of the aging and increasing population worldwide.

Spondylolisthesis can be one of the causes of low back pain. A patient suffers from spondylolisthesis when the upper vertebra of a segment partially slips over the lower vertebra. As a result, the spinal column loses stability and nerve roots can become compressed. When conservative measures such as physical therapy, pharmacological therapy (pain medication), and/or bracing do not have the desired effect, spinal surgery may be considered to stabilize the spine and/or to decompress nerve roots. Posterior Lumbar Interbody Fusion (PLIF) is a surgical procedure in which stabilization of spinal segments is achieved by means of pedicle screw instrumentation and interbody cages filled with bone graft. Approximately $30 \%$ of patients that undergo PLIF suffer from persistent or recurrent pain after surgery. The diagnosis of a patient with persistent or recurrent pain after PLIF includes review of medical history, clinical examination and imaging in order to elucidate the cause of pain. Patient reported outcome measures (PROMs) are validated questionnaires that can be used to quantify the burden of symptoms of a patient in terms of pain, loss of function or quality of life. Imaging can be used to confirm or rule out conditions that can cause pain. Common imaging modalities used in patients after PLIF include plain radiography, computed tomography (CT) and magnetic resonance imaging (MRI). These modalities can assess the anatomical result of a spinal fusion procedure, such as: fusion, the successful bony bridging between the operated vertebrae; pseudarthrosis, incomplete fusion; subsidence, defined as interbody cage migration; screw loosening or screw breakage. However, successful anatomical result after surgery does not automatically guarantees a pain free patient, neither is an anatomical abnormality necessarily associated with symptoms, which complicates diagnosis and treatment delineation. 
Nuclear medicine techniques focus on imaging of molecular and cellular processes in the human body rather than on visualizing anatomical structures. Positron Emission Tomography (PET) is a nuclear imaging modality that uses the decay characteristics of radionuclides to image and quantify metabolic and perfusion processes in the human body. PET can be combined with CT to also allow for localization of these processes. PET/CT with the bone seeking tracer ${ }^{18} \mathrm{~F}$-fluoride enables localization and quantification of bone metabolism. Analysis and interpretation of ${ }^{18} \mathrm{~F}$-fluoride PET/CT scans can be performed either visually, semi-quantitatively by calculating the standardized uptake value (SUV) or by pharmacokinetic modeling. SUV is a semi-quantitative measure for bone metabolism. Pharmacokinetic modeling yields parameters related to bone formation, regional bone blood flow and bone mineral incorporation/osteoblastic activity. For visual inspection and SUV calculation, static scanning at a certain time point after tracer administration is sufficient. For pharmacokinetic modeling, the uptake of tracer from the blood to the bone needs to be recorded by dynamic scanning.

In this thesis, the use of ${ }^{18} \mathrm{~F}$-fluoride PET/CT scanning in patients early after spinal fusion as well as in patients with persistent or recurrent symptoms several years after fusion surgery, was evaluated. In early postoperative patients, we hypothesized that PET activity within the operated segment could be an early predictor of the bone fusion status on CT at a later stage. In patients with persistent pain, we hypothesized that PET/CT could identify possible sources of pain by locating areas of increased bone stress.

The specific objectives of this thesis were:

To provide an overview of the diagnostic accuracy of imaging modalities to detect pseudarthrosis after spinal fusion, with surgical exploration as reference standard

The gold standard for the diagnosis of pseudarthrosis after spinal fusion is surgical exploration, an invasive, costly and nowadays rarely used test which is not desirable or ethical in patients without symptoms. Therefore, imaging is commonly used in the follow-up of patients after spinal fusion to verify whether bony fusion has been achieved or whether pseudarthrosis has developed. A systematic literature review was performed in Chapter 2 to identify studies that evaluated the diagnostic accuracy of imaging modalities to detect pseudarthrosis after thoracolumbar spinal fusion with surgical exploration as reference standard. Studies that evaluated the modalities plain radiography, flexion extension radiography, $\mathrm{CT}$, scintigraphy, tomography, ultrasound and $\mathrm{PET} / \mathrm{CT}$ were identified and included in the review. Meta-analyses with the odds ratio as outcome measure were performed to summarize the results of the studies. CT was considered the most accurate non-invasive modality for the detection of pseudarthrosis after thoracolumbar spinal fusion. 
To investigate the relationship between ${ }^{18}$ F-fluoride PET/CT, CT and clinical status several years after PLIF in symptomatic patients and asymptomatic patients

In Chapter 3 and Chapter 4 spinal fusion patients were evaluated by means of ${ }^{18} \mathrm{~F}$-fluoride $\mathrm{PET} / \mathrm{CT}$ and findings were correlated to patients' symptoms as measured by PROMs. Asymptomatic patients as well as symptomatic patients were included in the study cohort to learn about "normal" bone metabolism values of a spinal segment after PLIF as well as deviations therefrom in symptomatic patients. In Chapter 3 , anatomical findings on CT and functional findings on ${ }^{18} \mathrm{~F}$-fluoride PET/CT in regions within the operated segment were correlated to PROMs. In Chapter 4, CT and PET/CT findings of the facet joints, pedicle screws and adjacent segments were taken into account additionally.

In both chapters we confirmed the variable relationship between anatomical findings on $\mathrm{CT}$ and symptoms of a patient measured by PROMs. The spinal structures for which biological PET/CT findings correlated best to PROM scores were the facet joints, the lower endplate of the operated segment and the screw surroundings. In asymptomatic patients, we observed that a spinal fusion segment showed increased bone metabolism values in the adjacent facet joints and surrounding the screws to a certain extent, inherent to the surgical procedure performed. Deviating high increases in bone metabolism of facet joints, lower endplate of the operated segment and screw surroundings can point to pain generators in spinal fusion patients with persisting symptoms. Furthermore, we found an inverse correlation between fusion on CT and bone metabolism activity in the operated segment on PET/CT, as well as a linear correlation between the amount of subsidence on $\mathrm{CT}$ and the bone metabolism activity in the facet joints. From Chapters 3 and 4 , we concluded that functional information from ${ }^{18} \mathrm{~F}$-fluoride PET/CT scans to detect abnormalities in bone metabolism values at specific locations can be an important complement to the anatomical information from CT in the evaluation of symptomatic spinal fusion patients.

To develop an analysis method for dynamic ${ }^{18} \mathrm{~F}$-fluoride PET/CT scans of patients after PLIF

CT allows visualization and quantification of callus formation and bony bridging between the involved vertebrae. However, since these morphological signs of fusion occur late in the normal course of bone healing, CT is of limited use in the early postoperative phase. $\mathrm{PET} / \mathrm{CT}$ may provide valuable information early after fusion surgery as changes in bone metabolism can precede morphological alterations. Especially pharmacokinetic modeling can be of value since besides a measure on bone metabolism, this yields parameters related to bone blood flow and exposed bone crystal surface; both processes that occur early in the normal course of bone healing. Before pharmacokinetic modeling could be applied to a prospective cohort of PLIF patients early after surgery, an analysis method 
was developed in a retrospective cohort of PLIF patients. The path of the tracer in the human body after administration can be visualized and quantified by means of dynamic scanning. Pharmacokinetic modeling to analyze dynamic scans requires an input function. The input function can either be obtained via blood sampling, or by an image derived input function. In Chapter 5 we proposed and validated an analysis approach in patients after PLIF to extract parameters related to bone formation, regional bone blood flow and bone mineral incorporation/osteoblastic activity from a 30-minute dynamic ${ }^{18} \mathrm{~F}$-fluoride $\mathrm{PET} / \mathrm{CT}$ scan with an image derived input function in the aorta as the arterial input.

To investigate the potential of a ${ }^{18} \mathrm{~F}$-fluoride PET/CT scan early after PLIF to predict fusion outcome at one year

In Chapter 6, twenty prospectively included PLIF patients underwent ${ }^{18} \mathrm{~F}$-fluoride PET/CT scanning at six weeks and at one year after surgery. A diagnostic CT scan at one year was used to assess whether the included patients attained solid fusion or suffered from pseudarthrosis. Bone metabolism values were obtained from the ${ }^{18} \mathrm{~F}$-fluoride PET/CT scans using the analysis method proposed in Chapter 5. Based on the results of PET/CT scans at six weeks after PLIF, we were able to show differences between patients that attained fusion and patients developing pseudarthrosis at one year. The most important finding was that six weeks after PLIF, the bone metabolic activity in the operated intervertebral disc space was significantly lower in patients who were categorized as pseudarthrosis at one year as compared to patients who attained solid interbody fusion one year postoperatively. By means of full pharmacokinetic analysis, deviating patterns between pseudarthrotic and fused segments were also shown in parameters related to bone blood flow and bone mineral incorporation. Pseudarthrotic segments exhibited lower bone blood flow towards the operated segment in comparison to fused segments six weeks postoperatively, which resulted in less bone mineral incorporation in the intervertebral disc space at one year.

To conclude, ${ }^{18} \mathrm{~F}$-fluoride PET/CT can be a useful addition in the follow-up of patients after lumbar spinal interbody fusion. In clinical practice, hotspots indicating possible pain generators can be detected in patients with persistent or recurrent symptoms by means of static ${ }^{18} \mathrm{~F}$-fluoride PET/CT scanning. Pharmacokinetic modeling can be applied in an early stage after spinal fusion to predict the fusion result at one year after surgery. The applicability of this information in clinical practice is limited as yet. However, the ability of pharmacokinetic modeling after ${ }^{18} \mathrm{~F}$-fluoride PET/CT to evaluate bone blood flow separately from general bone metabolism, provides insights into the spinal fusion process and offers research opportunities, especially in the early postoperative phase. The findings in this thesis could be strengthened by additional long-term follow-up visits and the findings could be further explored by the evaluation of other types of bone graft and cage designs. An animal model for spinal fusion could be used to evaluate bone metabolism over time 
by multiple ${ }^{18} \mathrm{~F}$-fluoride PET/CT measurements. The application of ${ }^{18} \mathrm{~F}$-fluoride PET/CT in orthopedic patients could be expanded to patients undergoing total hip arthroplasty to assess the activity of bone metabolism surrounding a prosthesis as an indicator of bone ingrowth. 



\section{Nederlandse Samenvatting}

Lage rugpijn is wereldwijd een groot probleem. Het is de voornaamste oorzaak van functionele beperkingen en ziekteverzuim, alsmede een grote economische last. Kosten die gemoeid zijn met lage rugpijn zijn zowel directe kosten (medische kosten), als indirecte kosten ten gevolge van ziekteverzuim. De verwachting is dat de gevolgen van lage rugpijn blijven verergeren door de wereldwijde bevolkingsgroei en toenemende vergrijzing.

Spondylolisthesis kan een oorzaak zijn van lage rugpijn. Een patiënt lijdt aan spondylolisthesis wanneer de bovenste wervel van een segment gedeeltelijk over de onderste wervel schuift. Als gevolg hiervan verliest de rug stabiliteit en kunnen zenuwwortels bekneld raken. Indien conservatieve behandeling in de vorm van fysiotherapie, pijnmedicatie en/of bracing niet het gewenste effect heeft, kan worden overgegaan tot een operatie om de wervelkolom te stabiliseren en/of om decompressie van de zenuwen te bewerkstelligen. Posterior Lumbar Interbody Fusion (PLIF) is een operatie met als doel het stabiliseren van spinale segmenten met behulp van pedikelschroeffixatie en kooitjes gevuld met bot graft die tussen de wervels geplaatst worden. Ongeveer 30\% van de patiënten die een PLIF operatie ondergaan, kampt met persisterende of terugkerende pijn na de operatie. Het stellen van een diagnose bij PLIF patiënten met pijn wordt gedaan op basis van de aard van de klachten, lichamelijk onderzoek en beeldvorming. Patient Reported Outcome Measures (PROMs) zijn gevalideerde vragenlijsten die gebruikt worden om de symptomen die een patiënt ervaart in termen van pijn, verlies van functie en kwaliteit van leven, te kwantificeren. Beeldvorming kan gebruikt worden om bepaalde aandoeningen die pijn kunnen veroorzaken uit te sluiten dan wel te bevestigingen. Veelgebruikte beeldvormende technieken bij patiënten na PLIF, zijn röntgenfoto's, computer tomografie (CT) en magnetische resonantie beeldvorming (MRI). Met deze beeldvormende technieken zijn anatomische afwijkingen te zien zoals pseudartrose, gedefinieerd als incomplete fusie; subsidence, gedefinieerd als migratie van de intervertebrale cage; en schroef loslating of schroef breuk. Echter, een technisch succesvol resultaat na de operatie geeft geen garanties voor een pijnvrije patiënt, noch gaat een anatomische afwijking altijd gepaard met klachten, wat het stellen van de juiste diagnose compliceert.

Beeldvormende technieken binnen de Nucleaire Geneeskunde richten zich op het in beeld brengen van moleculaire en cellulaire processen in het menselijk lichaam, in plaats van het in beeld brengen van anatomische structuren. Positron Emissie Tomografie (PET) is een beeldvormende techniek die het vervalmechanisme van radionucliden gebruikt om 
metabole en perfusie processen in het menselijk lichaam te visualiseren en te kwantificeren. PET kan gecombineerd worden met CT om deze processen tevens te lokaliseren. Als de botzoekende tracer ${ }^{18} \mathrm{~F}$-fluoride gebruikt wordt, is het mogelijk om met PET/CT scans het botmetabolisme te lokaliseren en te kwantificeren. Het analyseren en interpreteren van de ${ }^{18} \mathrm{~F}$-fluoride PET/CT scans kan op verschillende manieren gedaan worden: visueel, semi-kwantitatief door de Standardized Uptake Value (SUV) te berekenen of volledig kwantitatief door middel van farmacokinetisch modelleren. SUV is een semi-kwantitatieve maat voor bot metabolisme. Farmacokinetisch modelleren levert parameters op die gerelateerd zijn aan bot formatie, bloedtoevoer naar het bot en bot mineraal incorporatie/osteoblast activiteit. Een PET/CT scan kan statisch of dynamisch gemaakt worden. Een statische scan is voldoende om de beelden visueel te bekijken en om de SUV waarde te kunnen berekenen. Voor het doen van farmacokinetisch modelleren is een dynamische scan nodig waarbij het proces van opname van de tracer van aorta tot in het bot geregistreerd wordt.

In dit proefschrift wordt het gebruik van ${ }^{18} \mathrm{~F}$-fluoride PET/CT scans bij de opvolging van patiënten na een PLIF operatie geëvalueerd, zowel vroeg na de operatie als bij persisterende of terugkerende klachten een aantal jaren na de operatie. Vroeg na de operatie, verwachten we dat PET activiteit in het geopereerde segment een voorspeller kan zijn van de uiteindelijke fusie uitkomst. In patiënten met persisterende of terugkerende klachten jaren na de operatie, verwachten we dat PET/CT van toegevoegde waarde kan zijn in het aanwijzen van structuren in de rug die pijn kunnen genereren door locaties van een toename in bot stress te identificeren.

De specifieke doelen van dit proefschrift waren:

Een overzicht bieden van de diagnostische nauwkeurigheid van beeldvormende technieken die gebruikt worden om pseudartrose na een spinale fusie operatie vast te stellen, met operatieve verificatie als referentie

Operatieve verificatie is de gouden standaard om pseudartrose na een spinale fusie operatie vast te stellen, maar het is ook een invasieve en dure test die tegenwoordig nog maar zelden gebruikt wordt en tevens niet ethisch is in patiënten zonder symptomen. Beeldvormende technieken worden veelal gebruikt om te onderzoeken of een patiënt na een spinale fusie operatie leidt aan pseudartrose. Hoofdstuk 2 beschrijft een systematische review waarin studies geïncludeerd werden die patiënten na thoracolumbale spinale fusie diagnosticeerden met zowel een beeldvormende techniek, als met operatieve verificatie als gouden standaard. Studies die de beeldvormende technieken radiografie, flexie-extensie radiografie, CT, scintigrafie, tomografie, ultrasound en PET/CT als diagnostisch middel gebruikten werden geïncludeerd. Meta-analyses met de odds ratio als uit- 
komstmaat werden uitgevoerd om de resultaten van de studies samen te vatten. Uiteindelijk bleek CT de meest nauwkeurige niet-invasieve modaliteit om pseudartrose te detecteren na thoracolumbale spinale fusie.

Het onderzoeken van de relatie tussen ${ }^{18} \mathrm{~F}$-fluoride PET/CT, CT en de klinische status van een patiënt op een aantal jaren na PLIF in symptomatische en asymptomatische patiënten

In Hoofdstuk 3 en Hoofdstuk 4 werden spinale fusie patiënten geëvalueerd door middel van ${ }^{18} \mathrm{~F}$-fluoride PET/CT en de bevindingen werden gecorreleerd aan de symptomen van de patiënten gemeten met PROMs. Asymptomatische patiënten werden geïncludeerd in het studie cohort om een inzicht te krijgen in het "normale" bot metabolisme patroon van een wervelkolom na spinale fusie. Vervolgens kon in symptomatische patiënten gekeken worden naar afwijkingen van dit normale patroon. In Hoofdstuk 3 werden anatomische bevindingen op CT en functionele bevindingen op ${ }^{18} \mathrm{~F}$-fluoride PET/CT binnen het geopereerde segment bepaald en gerelateerd aan PROMs. In Hoofdstuk 4 werd er naast de regio's binnen het geopereerde segment, ook gekeken naar de facet gewrichten, de pedikel schroeven en de aangrenzende segmenten. In beide hoofdstukken werd het gebrek aan een relatie tussen anatomische afwijkingen op CT en symptomen van de patiënt bevestigd. Met behulp van ${ }^{18} \mathrm{~F}$-fluoride PET/CT werd aangetoond dat mensen met symptomen afwijkende activiteitsniveaus lieten zien in de regio's rond de facet gewrichten, de pedikel schroeven en/of de eindplaten van het geopereerde segment. In asymptomatische patiënten werd aangetoond dat de botmetabolisme activiteit rond de aangrenzende facet gewrichten en rond de schroeven hoger is dan in een niet geopereerd segment, inherent aan de uitgevoerde operatie. Afwijkend hoge waardes in de facet gewrichten, schroef omgeving of eindplaten van een geopereerd segment in symptomatische patiënten kan duiden op een pijn generator. Daarnaast werd een invers lineaire correlatie gevonden tussen de mate van fusie op CT en de warde van botmetabolisme activiteit in het geopereerde segment op PET/CT, alsmede een lineaire correlatie tussen de mate van subsidence en de waarde van botmetabolisme activiteit in de facet gewrichten. Uit hoofdstukken 3 en 4 werd geconcludeerd dat de functionele informatie van ${ }^{18} \mathrm{~F}$-fluoride PET/CT scans een belangrijke aanvulling kan zijn op de anatomische informatie die wordt gegeven door CT in de opvolging van symptomatische PLIF patiënten.

Het ontwikkelen van een analysemethode voor dynamische ${ }^{18} \mathrm{~F}$-fluoride PET/CT scans van patiënten na PLIF

$\mathrm{CT}$ is in staat de callus formatie en bot brug vorming tussen wervels na een spinale fusie operatie te visualiseren en te kwantificeren. Aangezien deze morfologische tekenen van fusie pas laat in het normale bot heling proces optreden, is de waarde van CT gelimiteerd in de vroeg postoperatieve fase. PET/CT zou vroeg $n$ de operatie waardevolle informatie 
kunnen toevoegen aangezien veranderingen in het bot metabolisme ten grondslag liggen aan morfologische veranderingen. Farmacokinetische modelleren in het bijzonder kan van toegevoegde waarde zijn in de vroeg postoperatieve fase. Farmacokinetisch modeleren levert naast een parameter gerelateerd aan het botmetabolisme, ook parameters op die gerelateerd zijn aan de bloedtoevoer naar het bot en het beschikbare botkristal oppervlak, welke twee belangrijke processen zijn die vroeg in het verloop van bot heling optreden. Voordat farmacokinetisch modeleren toegepast kon worden in een prospectief PLIF cohort vroeg na de operatie, werd de analyse methode ontwikkeld in een retrospectieve PLIF patiënten groep. Voor farmacokinetisch modelleren is een input functie nodig welke gemeten kan worden door tijdens de scan bloed af te nemen of vanuit de dynamische PET scan. Hoofdstuk 5 beschrijft een analysemethode in patiënten na een PLIF operatie die een dynamische ${ }^{18} \mathrm{~F}$-fluoride PET/CT scan hebben ondergaan. Met behulp van de methode worden parameters die gerelateerd zijn aan botmetabolisme, bloedtoevoer naar het bot en bot mineraal incorporatie gemeten, gebruikmakend van een input functie in de aorta uit het dynamische PET beeld.

Het evalueren van de potentie van een ${ }^{18}$ F-fluoride PET/CT scan vroeg na een PLIF operatie om het fusie resultaat één jaar later te voorspellen

In Hoofdstuk 6 werden twintig patiënten prospectief geïncludeerd in een studieopzet waarin op zes weken en op één jaar na de operatie een ${ }^{18} \mathrm{~F}$-fluoride PET/CT scan werd gemaakt. Een diagnostische CT scan op één jaar na de operatie werd gemaakt om te bepalen of de patiënten fusie hadden bereikt of pseudartrose aan het ontwikkelen waren. Botmetabolisme waardes werden uit de ${ }^{18} \mathrm{~F}$-fluoride PET/CT scan gehaald door middel van de analyse methode die werd beschreven in Hoofdstuk 5. Gebaseerd op de resultaten van de PET/CT scans op zes weken na de operatie werden er verschillende patronen aangetoond worden voor patiënten die pseudartrose ontwikkelden in vergelijking met patienten die fusie ontwikkelden. De belangrijkste bevinding was dat de botmetabolisme activiteit op zes weken na de operatie in de geopereerde discus significant lager was in patiënten die pseudartrose ontwikkelden vergeleken met de patiënten die fusie ontwikkelden. Met behulp van farmacokinetisch modelleren was het mogelijk om verschillende patronen in bloedtoevoer naar het bot en in bot mineraal incorporatie tussen patiënten met en zonder fusie aan te tonen. In vergelijking tot gefuseerde segmenten, lieten pseudartrose segmenten een lagere bloedtoevoer naar het bot zien in het geopereerde segment, wat op één jaar resulteerde in verminder bot mineraal incorporatie in de tussenwervelschijf.

Concluderend, ${ }^{18}$ F-fluoride PET/CT kan een nuttige aanvulling zijn bij de follow-up van patiënten na lumbale spinale interbody-fusie. In de klinische praktijk kunnen hotspots die wijzen op mogelijke pijngeneratoren worden gedetecteerd bij patiënten met aanhoudende of terugkerende symptomen door middel van statische ${ }^{18} \mathrm{~F}$-fluoride PET/CT-scans. 
Farmacokinetische modellering kan in een vroeg stadium na spinale fusie worden toegepast om het fusieresultaat te voorspellen op één jaar na de operatie. De toepasbaarheid van deze informatie in de klinische praktijk is tot nu toe beperkt. Het vermogen van farmacokinetische modellering na ${ }^{18} \mathrm{~F}$-fluoride PET/CT om de botbloedsomloop afzonderlijk van het algemene botmetabolisme te evalueren, biedt echter inzicht in het spinale fusieproces en biedt onderzoeksmogelijkheden, vooral in de vroeg postoperatieve fase. De bevindingen in dit proefschrift kunnen worden versterkt door follow-up bezoeken op lange termijn en de bevindingen kunnen verder worden onderzocht door de evaluatie van andere soorten bottransplantaten en kooiontwerpen in nieuwe studies. Een diermodel voor spinale fusie zou gebruikt kunnen worden om het botmetabolisme in de loop van de tijd te evalueren door meerdere ${ }^{18} \mathrm{~F}$-fluoride PET/CT metingen te doen. De toepassing van ${ }^{18} \mathrm{~F}$-fluoride PET/CT bij orthopedische patiënten zou uitgebreid kunnen worden tot patiënten die een heupprothese krijgen om de activiteit van het botmetabolisme rondom een prothese te beoordelen als een indicator van botingroei. 



\section{List of Publications}

\section{JOURNAL PAPERS}

Peters MJM, Brans BT, Broos WAM, Jutten EMC, Visser MGW, Mottaghy FM, Schijns O, Weijers RE, Willems PC.

${ }^{18} \mathrm{~F}$-fluoride PET/CT to identify pain generators after lumbar spinal fusion - a retrospective analysis of symptomatic and asymptomatic patients

Submitted

Peters MJM, Brans BT, Wierts R, Jutten EMC, Broos WAM, Visser MGW, ter Laak MP, Mottaghy FM, Weijers RE, Willems PC.

${ }^{18} \mathrm{~F}$-fluoride PET/CT as an early predictor of bony fusion after posterior lumbar interbody fusion - a prospective study

Submitted

Verlaan L, Boekesteijn RJ, Oomen PW, Liu WY, Peters MJM, Emans PJ, van Rhijn LW, Meijer K.

Knee adduction moments are not increased in obsess knee osteoarthritis patients during stair negotiation.

Gait Posture 2019

Peters MJM, Bastiaenen CHG, Brans BT, Weijers RE, Willems PC.

The diagnostic accuracy of imaging modalities to detect pseudarthrosis after spinal fusion - a systematic review and meta-analysis of the literature.

Skeletal Radiology 2019

Verlaan L, Boekesteijn RJ, Oomen PW, Liu WY, Peters MJM, Witlox MA, Emans PJ, van Rhijn LW, Meijer K.

Biomechanical alterations during sit-to-stand transfer are caused by a synergy between knee osteoarthritis and obesity.

Biomed Res Int 2018

Peters M, Willems P, Weijers R, Wierts R, Jutten L, Urbach C, Arts C, van Rhijn L, Brans B. Pseudarthrosis after lumbar spinal fusion: the role of ${ }^{18} \mathrm{~F}$-fluoride PET/CT.

Eur J Nucl Mol Imaging 2015 
Peters MJ, Wierts R, Jutten EM, Halders SG, Willems PC, Brans B.

Evaluation of a short dynamic ${ }^{18}$ F-fluoride PET/CT scanning method to assess bone metabolic activity in spinal orthopedics.

Ann Nucl Med 2015

\section{CONFERENCE ABSTRACTS}

Peters MJM, Willems PC, Weijers RE, Wierts R, Jutten EMC, Urbach CJA, Arts JJC, van Rhijn LW, Brans BT

Clinical outcome after lumbar spinal interbody fusion in relation to ${ }^{18} \mathrm{~F}$-fluoride PET/CT scanning of the fusion region / Pseudarthrose na lumbale spondylodese: de rol van ${ }^{18} \mathrm{~F}$ fluoride PET/CT

Oral presentation at the Annual Meeting of the Dutch Orthopaedic Association (NOV) January 28-29 2016, 's Hertogenbosch, the Netherlands

Poster presentation at the Belgian Society of Nuclear Medicine (BELNUC) Biannual Symposium, May 9-10 2015, Maastricht, the Netherlands

Peters MJM, Brans BT, Wierts R, Jutten ECM, Mottaghy FM, van Rhijn LW, Willems PC.

${ }^{18} \mathrm{~F}$-fluoride $\mathrm{PET} / \mathrm{CT}$ as an early predictor of bony fusion after posterior lumbar interbody fusion.

Poster presentation at the North American Spine Society (NASS) Annual Meeting, September 24-29 2018, Los Angeles, USA

Poster presentation at the Orthopaedic Research Society (ORS) Annual Meeting, March 19-22 2017, San Diego, USA

'Best of Show' oral presentation at the EUROSPINE Annual Meeting, October 5-8 2016, Berlin, Germany

Oral presentation at the Annual Meeting of the European Orthopaedic Research Society (EORS), September 14-16 2016, Bologna, Italy

Peters MJM, Kok J, Arts JJC, Jutten EMC, ten Broeke RHM, Brans BT

A method to visualize and quantify bone mineralization around the cup after total hip arthroplasty longitudinally using ${ }^{18} \mathrm{~F}$-fluoride PET/CT scans

Poster presentation at the Belgian Society of Nuclear Medicine (BELNUC) Biannual Symposium, May 5-7 2017, Ghent, Belgium

Oral presentation at the European Federation of National Associations of Orthopaedics and Traumatology (EFORT) Annual Congress, May 27-29 2015, Prague, Czech Republic Poster presentation at the Orthopaedic Research Society (ORS) Annual Meeting, March 28-31 2015, Las Vegas, USA

Oral presentation at the Annual Meeting of the European Orthopaedic Research Society (EORS), July 2-4 2014, Nantes, France. 
Peters MJM, Kok J, Koster LA, Arts JJC, Jutten EMC, Vöö S, Brans BT, ten Broeke RHM ${ }^{18} \mathrm{~F}$-fluoride PET/CT to monitor bone ingrowth around acetabular cups after total hip arthroplasty

Oral presentation at the European Federation of National Associations of Orthopaedics and Traumatology (EFORT) Annual Congress, May 30-June 1 2018, Barcelona, Spain Poster presentation at the Orthopaedic Research Society (ORS) Annual Meeting, March 10-13 2018, New Orleans, USA

Best overall poster presentation at the Annual Meeting of the European Orthopaedic Research Society (EORS), September 13-15 2017, Munich, Germany

Jeuken RM, Peters MJM, Roth AK, van Rietbergen B, Emans PJ

${ }^{18} \mathrm{~F}$-sodium fluoride PET/CT as an imaging modality to evaluate initial fixation of focal knee resurfacing implants

Oral presentation at the Annual Meeting of the European Orthopaedic Research Society (EORS), September 13-15 2017, Munich, Germany

Peters MJM, Jeuken RM, Steijvers E, Wijnen W, Emans PJ

The modified Hedgehog technique to reattach chondral fragments in the young adult knee - follow-up with PROMs and 7.OT MRI at 3 months and 1 year after surgery

Oral presentation at the Annual Meeting of the European Orthopedic Research Society (EORS), October 2-4 2019, Maastricht, the Netherlands

Jeuken RM, Peters MJM, Vles GF, van Hugten PPW, Jansen EJP, Loeffen D, Emans PJ

The modified Hedgehog technique to repair pure chondral shear-off lesions in the pediatric knee

Poster presentation at the International Cartilage Repair Society (ICRS) World Congress, October 5-8 2019, Vancouver, Canada 



\section{Curriculum Vitae}

Marloes Peters was born on May 25th, 1988 in Arnhem, the Netherlands. She attended primary school in Huissen and graduated from the Over Betuwe College in Bemmel at pre-university level (VWO). Marloes moved to Eindhoven in 2006 to study Biomedical Engineering at the Eindhoven University of Technology. She performed an internship at the Human Performance Laboratory at the University of Calgary under supervision of Prof. dr. Benno Nigg. For her graduation project she did a project at the Maastricht University Medical Center with dr. Paul Willems and dr. Boudewijn Brans. She obtained her Master's degree in Medical Engineering from the Eindhoven University of Technology in 2013. Following the graduation project Marloes stayed in Maastricht to continue the ${ }^{18} \mathrm{~F}$ fluoride PET/CT research that amounted to her thesis. Currently she is working as a shared post-doctoral researcher fellow at the Maastricht University Medical Centerbetween the Complex Tissue Regeneration group of the MERLN Institute led by prof. dr. Lorenzo Moroni and the Department of Orthopedic Surgery of prof. dr. Lodewijk van Rhijn. 



\section{Dankwoord}

Poee, het is gelukt. Maar niet zonder jullie:

De leden van de beoordelingscommissie: prof. dr. M.W. de Haan, prof. dr. P. Habibovic, prof. dr. B.J. van Royen, dr. ir. B. van Rietbergen, dr. A.W.J.M. Glaudemans. Hartelijk bedankt voor de tijd en moeite die $u$ heeft genomen om mijn proefschrift te lezen en de bereidheid om zitting te nemen in de beoordelingscommissie.

Mijn promotor prof. dr. L.W. van Rhijn. Beste Lodewijk, bedankt voor de mogelijkheden die je me geboden hebt en voor de fijne en open sfeer die je creëert binnen de afdeling orthopedie. Bedankt voor je verhelderende oplossingen en bedankt voor de stok(ken) achter de deur(en) in de laatste fase van dit proefschrift.

Mijn copromotor dr. P.C. Willems. Beste Paul, je deur stond en staat altijd voor me open, dankjewel daarvoor. Je relativeringsvermogen en pragmatische oplossingen hebben nog al eens verhelderend gewerkt als ik de dingen weer eens ingewikkeld maakte. Bedankt voor de eindeloze versies van de stukken die je voor me hebt nagekeken. Mooi om samen het schapen avontuur aan te zijn gegaan.

Mijn copromotor dr. B.T. Brans. Beste Boudewijn, vrijdag 11:00 is niet meer hetzelfde sinds je niet meer in Maastricht werkt. Met hele bomen aan papier heb ik je vaak vermoeid met oneindige lijsten getalletjes. We moesten aan elkaar wennen, maar ik ben trots op de manier waarop we samengewerkt hebben. Bedankt voor alle inzichten die je me hebt gegeven.

Bedankt aan alle co-auteurs die hebben bijgedragen aan de stukken in dit proefschrift. Dr. Weijers, Beste René, hartelijk bedankt voor het beoordelen van al de CT scans en voor de tijd die u genomen heeft om de stukken in dit proefschrift uitvoerig te bekijken en te voorzien van waardevol commentaar. $U$ bent een onmisbare schakel geweest in dit proefschrift. Dr. ir. Wierts, Beste Roel, bedankt voor al je hulp op het technische vlak, erg fijn om een mede TU-er als back-up te hebben. Zonder jouw hulp was dit niet gelukt! Drs. Wouter Broos, bedankt voor de gezellige middagen rondjes tekenen en alle nucleaire en radiologische weetjes die je met me gedeeld hebt. Met wat goede zin en een hoop autodrop viel er best te werken met die software ;). Prof. dr. Mottaghy, Beste Felix, bedankt voor het meedenken in de orthopedische projecten. Mariëlle Visser, bedankt voor al het regelwerk aan nucleaire kant en het opstarten van alle projecten die we bedenken bij de 
orthopedie. Dr. Punt, Beste llona, bedankt voor de onderzoeksopzet waarmee dit onderzoek van start is gegaan! Ook dr. Carolien Bastiaenen, Liesbeth Jutten, dr. Chris Arts, dr. Mariel ter Laak en dr. Olaf Schijns, hartelijk bedankt voor jullie bijdrage als co-auteur.

Lino Aerts, Eefje Voets, Servé Halders, Christian Urbach, Cristel Demollin, en alle andere laboranten die PET/CT scans hebben gemaakt bij de patiënten (en/of schapen) beschreven in dit proefschrift, hartelijk bedankt. En ook de secretaresses van Nucleaire Geneeskunde, hartelijk bedankt voor de immer vrolijke begroeting en jullie hulp bij het inplannen van overleggen.

Alle medewerkers van CPV groot, Huub Souren, Joyce Suyk, Petra Dijkstra en Sanne Bout bedankt voor de fijne samenwerking en alle goede zorgen! Bedankt voor jullie bereidheid mee te werken aan alle projecten binnen de orthopedie en de onmogelijke constructies die we bedenken. Bedankt voor alle gezellige avonden scannen!

De studenten die gedurende het maken van dit proefschrift geholpen hebben bij het onderzoek. Meggy Verdonschot, bedankt voor je hulp bij het verwerken van al de schapen data, je was een stuk nauwkeuriger in het lab dan ik ooit had kunnen zijn. Joeri Kok, bedankt voor het opzetten van een methode voor de analyse van ${ }^{18} \mathrm{~F}$-fluoride PET/CT scans van de heup. Inmiddels is het stokje overgedragen aan Colet ter Voert die hard op weg is naar mooie resultaten. Bedankt voor jullie gedrevenheid en enthousiasme voor het ${ }^{18} \mathrm{~F}$ fluoride PET/CT onderzoek.

M'n reserve moeders van het trialbureau, bedankt voor het warme welkom dat jullie me aan het begin van dit onderzoek gegeven hebben en de fijne tijd samen op het trialbureau. Margareth, net als mijn eigen moeder is het jou ook niet gelukt om mij m'n bureau netjes te laten houden. Bedankt voor de fijne onderlinge pesterijen. Mijn (niet zo heel uitgebreide) Mestreechse vocabulaire heb ik aan jou te danken, ik doe m'n best! Anita, bedankt voor je rust, je grapjes en relativeringsvermogen, een fijne kamergenoot om te hebben! Liesbeth, bedankt voor het sparren over onderzoek en alles daaromheen. Mooi om te zien hoe je met je goede contacten binnen het ziekenhuis overal een pragmatische oplossing voor kan bedenken. Het trialbureau vervuld een aparte tak van sport binnen de orthopedie, die steeds belangrijker wordt!

Alle stafleden orthopedie: prof. dr. Lodewijk van Rhijn, dr. Paul Willems, dr. René ten Broeke, dr. Heleen Staal, dr. Peter Feczko, drs. Mark van den Boogaart, dr. Jan Geurts, drs. Joris Hermus, dr. Adhiambo Witlox, dr. Pieter Emans, dr. Tim Boymans en drs. Loek Verlaan. Bedankt voor jullie toegankelijkheid en voor de goede sfeer binnen de afdeling.

Denise, Astrid, Wil, Marion, Chantalle, Jerney, Manon, Helma, Mieke, Marieke en Caren hartelijk bedankt voor al jullie hulp en flexibiliteit, en voor de gezellige praatjes en vlaaimomenten. Denise, hartelijk bedankt voor alle voorbereidingen die je getroffen hebt om alles rondom het promoveren te regelen. 
Prof. dr. Lorenzo Moroni (ik doe het in het Nederlands ;)), bedankt voor de ruimte die je gegeven hebt om dit proefschrift af te maken. Je passie voor het uiteenlopende onderzoek binnen je groep werkt inspirerend en ik heb veel bewondering voor je werkmentaliteit. Dr. Pieter Emans, bedankt voor al je ideeën, wolken en je aanstekelijke enthousiasme. Op naar meer successen de komende tijd, ik ga m'n uiterste best doen om een paar ideeën te verwezenlijken.

Kim Feron, Job van den Hurk en Esther Steijvers, bedankt voor de fijne samenwerking bij Scannexus tot nu toe en op naar een hoop mooie dingen in de toekomst! Esther, gaan we in november nu eindelijk schoenen kopen?

Het lab van de orthopedie! Fijne omgeving om in te werken en te leren. Plek waar hard gewerkt wordt maar ook altijd gelegenheid is voor een lolletje en waar iedereen z'n eigen maffe trekjes mag hebben. De afgelopen jaren zodanig gegroeid dat er een hoop mensen zijn om te bedanken! Tim Welting, drijvende kracht achter het onderzoek op het lab. Bedankt voor je enthousiasme, nieuwsgierigheid, passie en trots. Mooi om te zien hoe veel professioneler en groter het lab de afgelopen jaren is geworden! Chris Arts, je nuttige bedrijven contacten zijn een mooie aanvulling voor het werk dat we doen op de universiteit, bedankt voor je enthousiasme en nieuwe ideeën. Bert van Rietbergen, leuk om de TU connectie warm te houden en bedankt voor je medewerking aan de afstudeerprojecten van de (B)ME-ers. Don Surtel en Andy Cremers, bedankt voor alle fijne hulp bij de praktische zaken. Mooi dat het duo een viertal geworden is en dat Laura Peeters en Jessica Steijns zich niet laten ondersneeuwen door jullie. Laura, ik heb zin om samen aan de slag te gaan met het nieuwe osteochondrale project! Raymond Bevers, heerlijk om een buurman te hebben die ik altijd mag pesten. Dat ik regelmatig ook de volle lading over me heen krijg neem ik dan graag voor lief. Bedankt voor je Inkscape tip! Alex Roth, ik bewonder je focus en efficiëntie, een echte harde werker die veel voor elkaar krijgt. Marjolein Caron, multi-tasken kan jij als de beste; onderwijs, onderzoek, studenten en de kids thuis, alles heb je onder controle. Kaj Emanuel, we zijn nog wel even bezig totdat we alle wilde ideeën uitgewerkt hebben, bedankt voor je enthousiasme en frisse blik. Remco Doodkorte, helaas is onze arbeid nog even niet in dit boekje terecht gekomen, maar ik vond het wel gezellige dagen, dat samen testen op Chemelot. Gelukkig heb je nu een heel wat mooier apparaat met minder frustrerende eigenschappen. Pieter van Hugten, leuk om met je samen te werken aan allerlei spannende zijprojectjes! Culinaire magnetron maaltijden mogen hierbij niet ontbreken. Arjan Loenen, heel fijn dat al het schapenwerk niet voor niks is geweest en dat jij nu ook met de schapen over de gang rond sjeest. Ik ben benieuwd wat eruit komt! Jasper Stevens en Tom van Vugt, altijd leuk om met jullie naar Spanje te gaan. Ik had me verheugd op goede paella en lekkere sangria, maar ik kreeg overheerlijke pasta, ik snap het nog steeds niet. Eva Jacobs, dit hebben we toch maar even mooi voor elkaar gebokst. Je zal wel weer boos worden als ik dit zeg, maar je bent echt intelligent en ik heb veel bewondering voor je drive. Mandy Steinbusch, er komt een eind aan het zwoegen, zet 'm op! Ralph Jeuken en Ufuk Tan Timur, het lab is niet 
meer hetzelfde sinds jullie de kliniek in zijn. Het is veel gezelliger, beter werkbaar etc. Nee geintje, jullie worden gemist! Het was een feest om een paar jaar geleden bij jullie op de kamer te zitten, ik mis het wel dat me niet meer elke tien minuten gevraagd wordt hoe het met me gaat. Maarten Janssen, leuk om samen te werken aan een hele spannende MRI studie en in het weekend gezellig te BBQ'en. Ellen Ripmeester, Nina Wijnands, Roderick Stassen, Bas Housmans, Alzbeta Chabronova, Guus van den Akker, Mirella Haartmans, Lotte Smals, bedankt!

Mariët van Duren - Koopman, m'n studiemaatje in Eindhoven, maar al snel veel meer dan dat! Hoe heerlijk is het om elkaar met een half woord te begrijpen. We zien elkaar niet meer zo vaak als vroeger, maar dat doet er niet toe, ik had me geen betere paranimf kunnen wensen. Ik heb m'n calculus boek nog bewaard hoor, dan kunnen we ten minste sommetjes maken als we later samen in het bejaardentehuis aan een kopje thee zitten. Mooie tijd hebben we gehad in het net niet witte dorp met ons $3^{e}$ nicasiuszusje Pauline Vrancken. Drie afstuderende meiden in één huis, erg fijn spuien als er één van ons iets dwars zat. Een echte feestdag als we met z'n drieën een thuiswerkdag hadden. Ik zou zo weer met jullie in één huis gaan wonen.

MASLMAIS: Maartje, Anne-Marie, Sanne, Lucie, Amber, Ingrid en Sietske, lieve meissies, wat is het altijd heerlijk om jullie weer te zien, voelt als thuiskomen. Elkaar begrijpen is makkelijk als je zo goed van elkaar weet waar je vandaan komt. Ook al zien we elkaar niet al te vaak, dat gaat niet meer weg! Mooi om te zien hoe iedereen op z'n eigen plekje terecht gekomen is :). Amber, een extra bedankje voor jou voor het meedenken over de cover!

De eetclub! Michiel \& Elske, Joep \& Fieke, Jasper \& Judith, Duncan \& Els, Bram \& Anouk (geen officiële leden, maar jullie horen wel in dit rijtje thuis) bedankt voor al jullie gevraagde en vooral ook ongevraagde advies. De tijd dat de eetclub elke maandag bijeenkwam voor een culinair hoogstandje is alweer even voorbij, maar altijd goed om jullie weer te zien!! Michiel, m'n bonusbroer, we maken er vaak grapjes om, maar bedankt dat je me een leven hebt gegeven :P ik had de gezellige hockeydagen en alles eromheen niet willen missen. Michiel en Bram, speciaal bedankje voor jullie voor alle bijlessen tijdens het studeren. Kan prima in de kroeg toch?! We hadden het eigenlijk ook net zo goed onder Twin Peaks kunnen doen, ik geloof niet dat ik dan nog slechter had begrepen waar de serie eigenlijk over gaat.

Koninklijke Harmonie Oosterbeek, ideale manier om m’n gedachten even te verzetten. Bedankt voor de vrijheid die jullie me geven en voor jullie oprechte interesse in het onderzoek! Mijn duim liet me even in de steek, maar I will be back. Bram Versteegen, bijzonder hoe jij door de muziek heen kan horen of me iets dwars zit. Bedankt voor alle gezellige lessen. Mooi om te zien dat je je pad helemaal gevonden hebt in de muziek. 
Oranje-Rood Dames 6, samen met jullie promoveer ik een stuk sneller dan op de universiteit en vaker ook. Ons team is een mooie combi van fanatisme op het veld en een hoop gezelligheid daarnaast, dat is me het ritje wel waard. Ik geniet van alle uitjes, teamweekenden en natuurlijk het jaarlijkse PietJanKnor avontuur. Speciaal bedankje voor de space shuttles, Pauline, Imke en Fabiënne, voor de gezellige weekendjes weg en alle lol die we samen hebben.

Ruud, Ciska, Ivan, Maaike, Mats, Kasper, Kristel en Jos, bedankt voor het warme welkom in jullie gezellige en actieve familie.

En dan mijn eigen Huissense familie :) Rick, aangezien ik in m'n eentje naar Eindhoven ging, was het erg fijn om jou daar als grote broer achter de hand te hebben. Zo kon ik altijd bij je terecht als er dingen niet lukte, van het koken van pasta voor acht man, tot de sommetjes bij calculus. Daarom een fijn en veilig gevoel om je bij me te hebben als paranimf! Inge, inmiddels ook een echte Peters! Goed om je erbij te hebben en mooi om te zien hoe goed jullie het voor elkaar hebben met zn drietjes in Eindhoven :). Mees! Hoewel er nog maar weinig aandacht over blijft voor ons als kinderen, is het heerlijk om jou erbij te hebben en het wordt steeds leuker! Anouk, één studie vond je niet genoeg. Met inmiddels heel wat diploma's op zak zit je nog steeds niet stil en ben je telkens weer op zoek naar een nieuwe uitdaging. Je doorzettingsvermogen maakt me trots. De frequentie van onze zussendagen moeten we weer even opvoeren hoor! Opa \& Oma, bedankt voor jullie interesse in alles wat ik doe. Jullie zijn twee echte bikkels en ik heb veel bewondering voor jullie. Lieve papa \& mama, hoe kan ik jullie bedanken. Ik had niet gedacht dat ik zou gaan promoveren, vroeger niet, maar een jaar geleden zeker ook niet ;). Op een gegeven moment hadden jullie door dat je het beste niet meer kon vragen naar de voortgang van dit boekje, maar nu is ie dan toch af. Bedankt voor jullie luisterend oor, goede raad en vooral rust en eerlijkheid. De uitspraak "die mensen moeten ook allemaal naar de wc hoor Marloes" heeft me vaak geholpen als ik zenuwen had. Petersss powerrr.

Alex, ik weet ook niet hoe, maar het is gelukt. Dankjewel voor alles, je weet niet half hoe blij ik met je ben. 
